\title{
Energy balance and colorectal cancer : understanding disease pathways through molecular epidemiology
}

Citation for published version (APA):

Simons, C. C. J. M. (2013). Energy balance and colorectal cancer : understanding disease pathways through molecular epidemiology. [Doctoral Thesis, Maastricht University]. Maastricht University. https://doi.org/10.26481/dis.20131216cs

Document status and date:

Published: 01/01/2013

DOI:

$10.26481 /$ dis.20131216cs

Document Version:

Publisher's PDF, also known as Version of record

\section{Please check the document version of this publication:}

- A submitted manuscript is the version of the article upon submission and before peer-review. There can be important differences between the submitted version and the official published version of record.

People interested in the research are advised to contact the author for the final version of the publication, or visit the DOI to the publisher's website.

- The final author version and the galley proof are versions of the publication after peer review.

- The final published version features the final layout of the paper including the volume, issue and page numbers.

Link to publication

\footnotetext{
General rights rights.

- You may freely distribute the URL identifying the publication in the public portal. please follow below link for the End User Agreement:

www.umlib.nl/taverne-license

Take down policy

If you believe that this document breaches copyright please contact us at:

repository@maastrichtuniversity.nl

providing details and we will investigate your claim.
}

Copyright and moral rights for the publications made accessible in the public portal are retained by the authors and/or other copyright owners and it is a condition of accessing publications that users recognise and abide by the legal requirements associated with these

- Users may download and print one copy of any publication from the public portal for the purpose of private study or research.

- You may not further distribute the material or use it for any profit-making activity or commercial gain

If the publication is distributed under the terms of Article $25 \mathrm{fa}$ of the Dutch Copyright Act, indicated by the "Taverne" license above, 
Energy balance and colorectal cancer: understanding disease pathways through molecular epidemiology 
(C) Maastricht 2013, Colinda C.J.M. Simons

Energy balance and colorectal cancer: understanding disease pathways through molecular epidemiology

Key words: colon neoplasms; cohort studies; energy balance; gene-environment interactions; genetic instability; insulin-like growth factors; molecular epidemiology; methylation; overweight; rectal neoplasms; single nucleotide polymorphisms; tumor heterogeneity

ISBN 978-90-821308-0-5

Layout: Colinda Simons en Jos Bruystens, Maastricht

Cover: Eemie Illustration, Den Bosch

Printed by: Printservice, Ede

Financial support for the printing of this thesis was kindly provided by the Dutch Cancer Society and Sequenom, GmbH.

All rights reserved. No part of this thesis may be reproduced or transmitted in any form or by any means, electronic or mechanical, including photocopying, recording or any information storage or retrieval system, without permission in writing from the author, or, when appropriate, from the publishers of the publications. 


\title{
Energy balance and colorectal cancer: understanding disease pathways through molecular epidemiology
}

\author{
PROEFSCHRIFT
}

Ter verkrijging van de graad van doctor aan de Universiteit Maastricht, op gezag van de Rector Magnificus, Prof. dr. L.L.G. Soete,

volgens het besluit van het College van Decanen, in het openbaar te verdedigen op maandag 16 december 2013 om 16.00 uur

door

Colinda Cornelia Johanna Maria Simons Geboren op 14 december 1983 te Roermond 


\section{Promotoren:}

Prof. dr. M.P. Weijenberg Prof. dr. M. van Engeland

Prof. dr. F.J. van Schooten

\section{Beoordelingscommissie:}

Prof. dr. A.A.M. Masclee (voorzitter)

Prof. dr. E.E. Blaak

Prof. dr. R. Kaaks (German Cancer Research Center, Heidelberg)

Dr. S.W.M. Olde Damink

Prof. dr. M.P. Zeegers

This PhD research was supported by the Dutch Cancer Society (KWF), the Health Foundation Limburg and the Biobanking and Biomolecular Research Infrastructure in the Netherlands (BBMRI-NL).

The studies presented in this thesis were conducted at the GROW-School for Oncology and Developmental Biology at Maastricht University (departments of Epidemiology and Pathology) in collaboration with the NUTRIM - School for Nutrition, Toxicology and Metabolism (department of Toxicology). 


\section{-CONTENTS}

Chapter 1 General introduction

Epidemiology - diet and lifestyle

Chapter 2 Body size and colorectal cancer risk after 16.3 years of follow-up: an analysis from the Netherlands Cohort Study

Chapter 3 Physical activity, occupational sitting time, and colorectal cancer risk in the Netherlands Cohort Study

Molecular epidemiology - genes and environment

Chapter 4 Energy balance and unfavorable alleles in genes related to the IGF pathway jointly influence colon cancer risk in men

Molecular (pathologic) epidemiology - tumor heterogeneity

Chapter 5 Energy balance influences the risk of having a colorectal tumor with methylated insulin-like growth factor binding protein genes

Chapter 6 Body size, physical activity and risk of colorectal cancer with or without the CpG island methylator phenotype (CIMP)

A novel classification of colorectal tumors based on microsatellite

Chapter 7 instability, the CpG island methylator phenotype and chromosomal instability: implications for prognosis

Chapter 8 General discussion

Summary/Samenvatting

Etcetera

(Dankwoord, about the author, list of publications) 



\title{
General introduction
}

\author{
Colinda CJM Simons
}


Cancer begins and ends with people. In the midst of scientific abstraction, it is sometimes possible to forget this one basic fact...

$$
\text { - June Goodfield }
$$

\subsection{Colorectal cancer}

Globally, in the year 2008, colorectal cancer (CRC) affected more than 1.2 million new individuals, thereby ranking third on the list of most common cancers in men and women combined. In the same year, the agestandardized mortality rate was 8.2 per 100,000 person-years, accounting for $8.1 \%$ of all cancer deaths. ${ }^{1}$ Factors for which there is substantial evidence that these increase risk include a family history of $\mathrm{CRC},{ }^{2}$ body fatness, adult attained height, a lack of physical activity, meat intake, processed meat intake, alcohol use ${ }^{3}$ and smoking. ${ }^{4,5}$ Most of these risk factors increase risk only modestly. To identify potential subgroups particularly at-risk and work towards better prevention strategies, an enhanced understanding of the disease pathways linking dietary and lifestyle factors to CRC is necessary. This task is not easy as CRC has emerged as a very complex disease, which is indicated by that risk factors differ between men and women ${ }^{6}$ and for cancers in different sublocalizations in the colorectal tract.7,8 Moreover, colorectal tumors display specific morphological and molecular characteristics, which correlate with the site of manifestation and which may influence prognostic outcomes. ${ }^{9,10}$ Therefore, the adenoma-carcinoma sequence, which is used to describe CRC development, appears to be instigated by different risk factors and takes different developmental routes, resulting in distinct tumor appearances and prognostic outcomes (Box 1).

\subsubsection{Energy balance}

Indicators of energy balance, which include body mass index, waist circumference and physical activity, are established risk factors for CRC, particularly colon cancer. ${ }^{3}$ Energy balance is defined as the ability to maintain one's body weight by balancing energy intake with energy expenditure. ${ }^{11}$ Energy intake is determined by the number of calories consumed from foods, drinks and alcohol; energy expenditure is determined by the level of physical activity and the basal metabolic rate (i.e. the amount of energy needed to sustain the activities of vital organs). ${ }^{12}$ Mechanisms linking energy balance to CRC risk are thought to involve insulin-like growth factors, inflammation/immune responses, and hormones. ${ }^{13-15}$ Despite the growing interest in indicators of energy balance and CRC, observational evidence is limited with respect to energy balance and the risk of CRC in specific anatomical subsites (the proximal colon, distal colon, rectosigmoid and rectum, Box 1) and with respect to the pathways underlying these associations.

\subsubsection{The insulin-like growth factor pathway}

The insulin-like growth factor (IGF) pathway is a putative link between energy balance and CRC because it is central to regulating growth in the human body, including cancer growth, and it is influenced by disturbances in energy balance. ${ }^{16}$ Important components of the IGF system include IGF-1, the main growth factor in adult life, and IGF binding proteins (IGFBPs), of which the main function is to sequester IGFs, thereby downregulating IGF bioavailability (thus inhibiting growth). ${ }^{15,17}$ Increased IGF-1 and reduced IGFBP levels have been associated with prolonged hyperinsulinaemia, which results from a state of insulin resistance. ${ }^{18}$ Insulin resistance is defined as an impaired biological response to the action of insulin, and can ultimately lead to type 2 diabetes mellitus. Of interest, type 2 diabetics have been observed to be at an increased risk of CRC. ${ }^{19}$ 
Box 1. A) The distribution of colorectal cancers by anatomical subsite. B) The adenomacarcinoma sequence. C) Common molecular alterations in colorectal cancer.

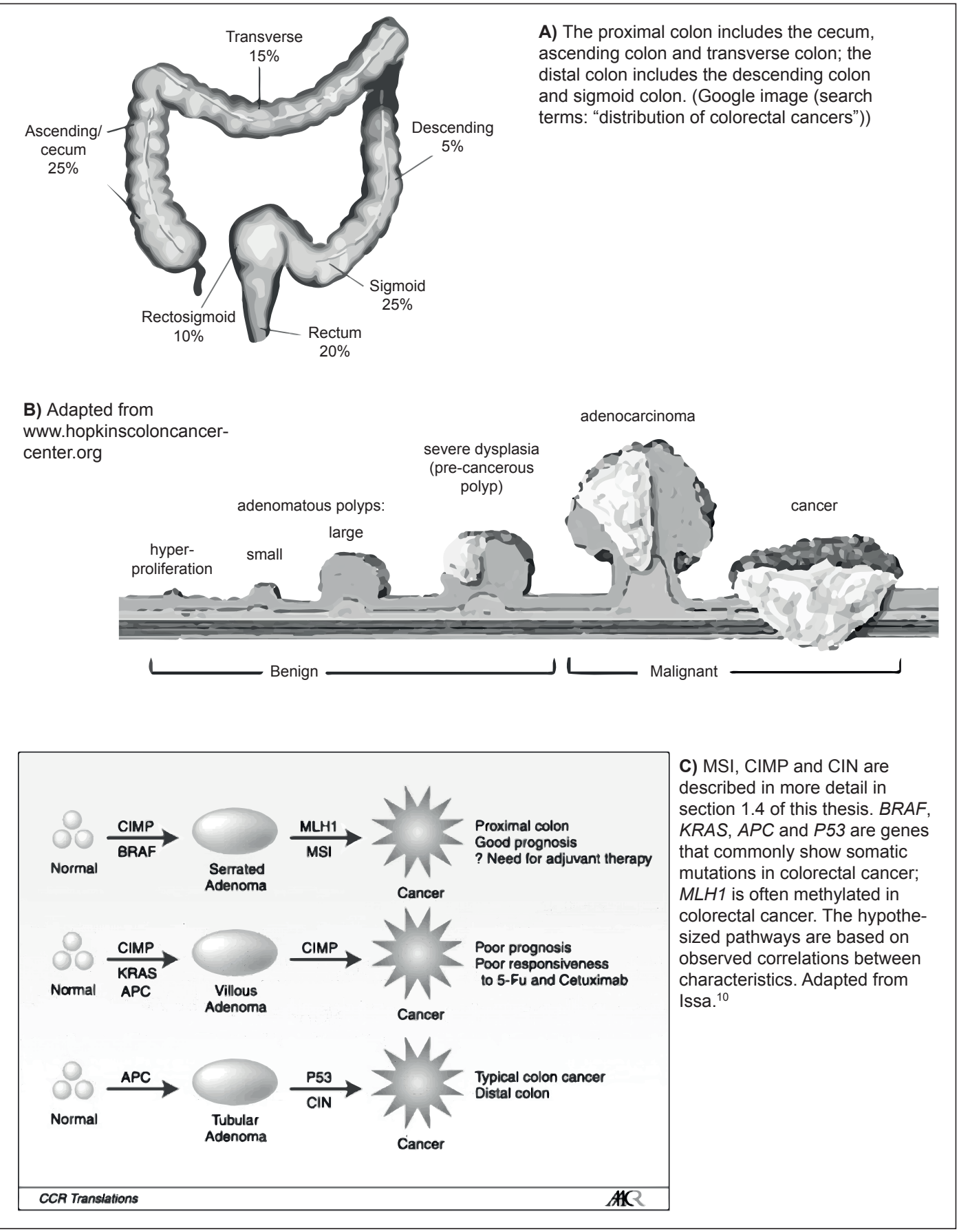




\subsection{Molecular epidemiology}

In order to shed light on the pathways linking energy balance to CRC risk, a molecular epidemiological approach has been applied in this thesis. Molecular epidemiology has been referred to as the inclusion in epidemiologic research of biologic measurements made at the molecular level. ${ }^{20}$ In this thesis, genetic variation was measured in genes in the IGF pathway and in closely related genes. Combined effects of these variants and indicators of energy balance were studied in relation to CRC risk. The selected genes of interest encoded for the following factors: growth hormone releasing hormone (GHRH), growth hormone releasing hormone receptor (GHRHR), growth hormone 1 (GH1), growth hormone receptor (GHR), insulin-like growth factors 1 and 2 (IGF1-2), insulin-like growth factor binding proteins 1 through 7 (IGFBP17), IGFBP acid labile subunit (IGFALS), IGF1 and -2 receptors (IGF1-2R), insulin receptor substrates 1 and 2 (IRS1-2), peroxisome proliferator-activated receptor gamma (PPARG), adiponectin (ADIPOQ), and adiponectin receptors 1 and 2 (ADIPOR1-2). In addition to studying genetic variants, we studied specific molecular tumor subtypes in CRC as markers of etiological pathways. This is sometimes referred to as molecular pathologic

epidemiology. ${ }^{21}$

\subsection{Gene-environment interactions}

To aid further discussion on the study of combined effects of genetic variants and indicators of energy balance and potential geneenvironment interactions, some relevant concepts will be elaborated on in the next subsections.

\subsubsection{Genetic variation: the basics}

DNA is made up of two strands consisting of sugar-phosphate groups and four nucleotide bases: adenosine $(\mathrm{A})$, thymine $(\mathrm{T})$, cytosine $(\mathrm{C})$ and guanine $(\mathrm{G})$. The two strands form a double helix - much like a winding staircase - in which adenosine always pairs with thymine, and cytosine with guanine. In total, there are about 3.3 billion base pairs that can be mapped to 23 homologous pairs of chromosomes (one of maternal and one of paternal origin) which contain some 25,000 genes (stretches of DNA that typically code for one protein). ${ }^{22}$

Genetic variation between individuals exists in approximately $1 \%$ of the DNA. Genetic variation may be of the type insertions/deletions of larger stretches of DNA, variations in the number of repeats in repeated sequences in the DNA, and single-base pair changes. Single base-pair changes are the most common type of genetic variation, termed single nucleotide polymorphisms (SNPs; pronounced 'snips'). ${ }^{22}$ Most SNPs have two alleles, i.e. two different bases are known to occur across individuals in the same locus, of which the allele that occurs at lowest frequency in a population is called the minor allele. If an individual carries the minor allele on both parental chromosomes, he/she is said to be homozygous for the rare allele, as opposed to being heterozygous (carrying both alleles) or homozygous for the common allele.

\subsubsection{SNP functionality}

The functionality of a SNP is often ambiguous because there exists a cascade of events between the SNP and there where there may be effects on health and disease (Figure 1). The location of a SNP in the genome may give a first hint about the likelihood that a SNP has functional properties. In this respect, coding regions and non-coding regions are discerned. Coding regions contain exons and introns which, together with untranslated regions at both ends, are transcribed into RNA. Before translation to a protein, introns are spliced 
from the RNA (Figure 2). The specific amino acids that assemble the protein are each encoded for by three subsequent base pairs in the exons (codons). These exons may contain SNPs of the type non-sense (stopping translation), missense (introducing an amino acid change), and sense (introducing no amino acid change). Non-sense and missense SNPs are collectively referred to as nonsynonymous SNPs, of which functional effects are thought likely. As a result, it has been common to study nonsynonymous SNPs in relation to disease. However, exons do not always contribute to the end product, because these may be spliced in alternative splicing processes. Alternative splicing leads to the existence of different isoforms of the same protein, and may be tissue-dependent. ${ }^{23}$ With respect to the genes selected for study in this thesis, splice variants have been reported to exist for almost all. ${ }^{23-26}$

The above is not to indicate that SNPs in introns or intergenic regions cannot be functional. In contrast, most hits in studies that agnostically scanned hundreds of thousands of SNPs in the genome for SNP-disease associations (referred to as genome-wide association studies, GWAS) were intergenic SNPs. ${ }^{27}$ Possibly, these regions harbour many loci with important regulatory functions.

\subsubsection{Linkage disequilibrium}

Linkage disequilibrium (LD) is defined as the non-independence of alleles at different loci. For example, if allele A at locus 1 and allele B at locus 2 occur at frequencies $\mathrm{pA}$ and $\mathrm{pB}$ in the population, respectively, one would expect to see the $A B$ haplotype at frequency
$\mathrm{pApB}$ if the alleles are independent. If the population frequency of the $\mathrm{AB}$ haplotype differs from this, then the two loci are said to be in LD (thus, particular alleles tend to be observed together). ${ }^{28}$ LD structures may differ between chromosomal regions and populations. ${ }^{29}$ As a consequence of LD, SNPs found associated with disease are often not the causal variants themselves, but markers for the causal variants.

\subsubsection{Concepts of interaction}

Statistical interaction refers to departure from multiplicativity of risk ratios or additivity of risk differences. Since it is tested whether the observed combined effect differs from the expected combined effect, statistical interaction requires there to be main effects of the gene and the environment. However, this may not equal what constitutes interaction on the biological level. ${ }^{30,31}$ Biological interaction has been defined as the participation of two component causes in the same sufficient cause by Rothman and Greenland. ${ }^{31,32}$ This definition implies that the gene and the environment may lack effects in the absence of the other. Khoury et al. ${ }^{33}$ summarized gene-environment interaction patterns in which this becomes clear (Table 1). A classic example is the interaction between the NAT2 gene and smoking, where NAT2 slow versus rapid/intermediate acetylators are at an increased risk of bladder cancer only among cigarette smokers. ${ }^{34}$ A rare type of interaction would involve crossover (qualitative interaction), where the effect from one factor is reversed by the presence of another, e.g. a higher body mass index reduces breast cancer risk before the menopause but increases it after the menopause. ${ }^{35}$ 


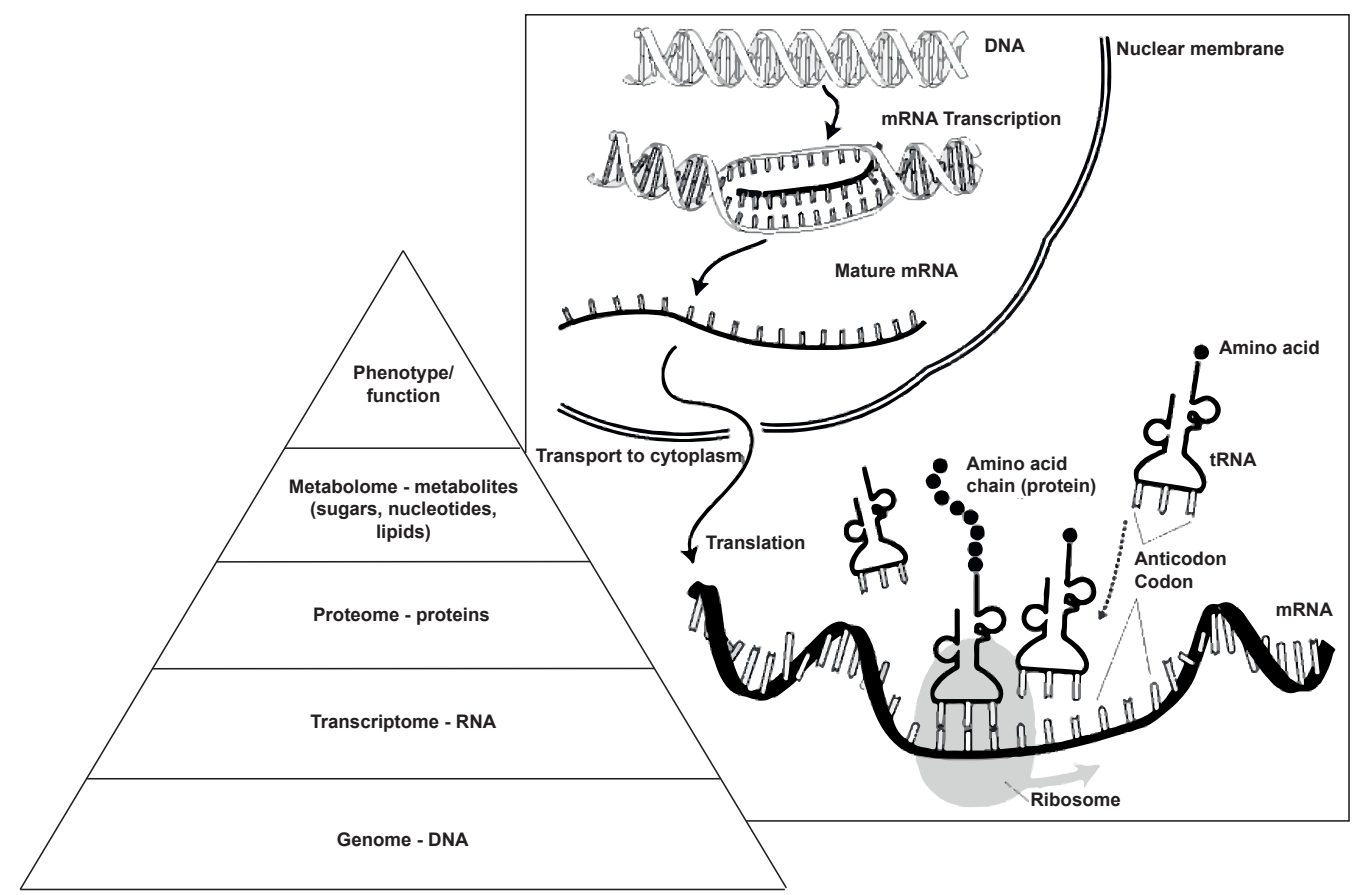

Figure 1. From the human genome to phenotypes and functions through transcription of DNA into RNA, translation of RNA into proteins, and protein-metabolite interactions (Google image (search terms: "transcription and translation")).

Abbreviations: DNA, deoxyribonucleic acid; mRNA, messenger ribonucleic acid; tRNA, transfer ribonucleic acid.

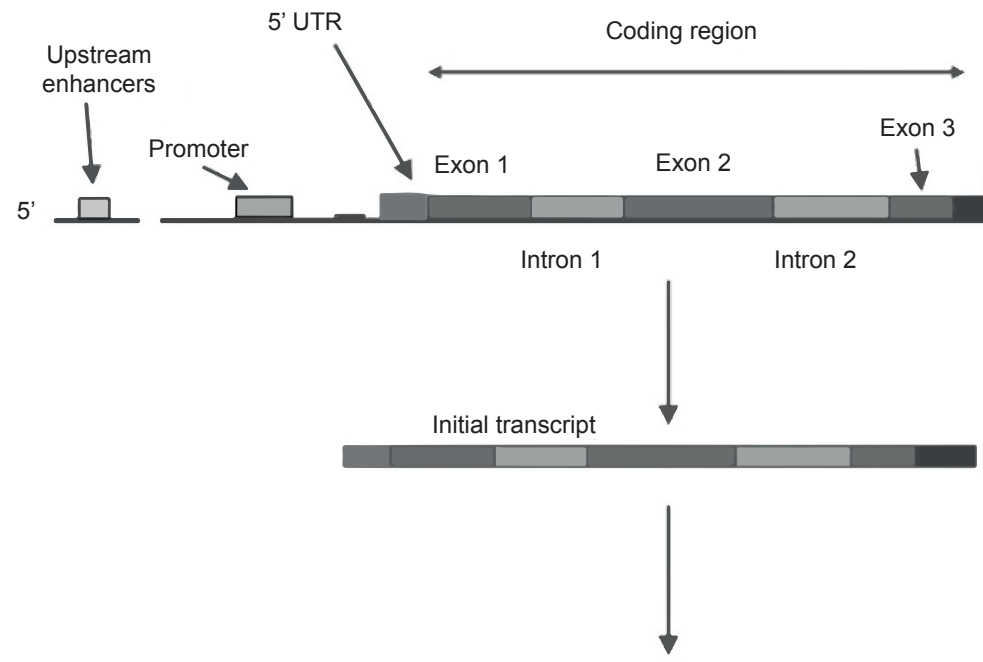

Final mRNA

Figure 2. Typical gene structure and RNA splicing. (Google image (search terms: "gene structure")).

Abbreviations: DNA, deoxyribonucleic acid; mRNA, messenger ribonucleic acid; UTR, untranslated region. 
Table 1. Patterns of gene-environment interaction, adapted from Khoury et al. ${ }^{33}$ Patterns in which the direction of effects, if any, is reversed are also possible

\begin{tabular}{ccc}
\hline Pattern $^{*}$ & Genotype in the absence of environment & Environment in the absence of genotype $^{*}$ \\
\hline 1 & No effect, $R_{g}=1$ & No effect, $R_{e}=1$ \\
2 & No effect, $R_{g}=1$ & Increased risk, $R_{e}>1$ \\
4 & Increased risk, $R_{g}>1$ & No effect, $R_{e}=1$ \\
5 & Increased risk, $R_{g}>1$ & Increased risk, $R_{e}>1$ \\
6 & Decreased risk, $R_{g}<1$ & No effect, $R_{e}=1$ \\
\hline \hline
\end{tabular}

${ }^{*} \mathrm{R}_{\mathrm{e}}=$ disease risk among persons with exposure but without genotype, divided by the disease risk among persons with no exposure and no susceptible genotype; $\mathrm{R}_{\mathrm{g}}=$ disease risk among persons with genotype but without exposure, divided by the disease risk among persons with no exposure and no susceptible genotype.

\subsection{Molecular signatures in tumors}

Next to the study of GxE interactions, clarification of the associations between indicators of energy balance and the risk of CRC by tumor subtype may lead to a better understanding of the pathways linking energy balance to CRC. Acquired (epi)genetic alterations in the tumor can be viewed as a molecular signature, identifying the tumor's developmental route.

\subsubsection{Epigenetic instability}

Epigenetic changes are changes in gene expression that are independent of changes in the DNA sequence. ${ }^{36,37}$ Promoter hypermethylation of DNA is a common epigenetic alteration that entails the binding of methyl groups to $\mathrm{CpG}$ rich regions in the promoter of a gene ('CpG islands'). Promoter CpG island hypermethylation has been shown present in tumor suppressor- and DNA repair genes in colorectal tumors, and is associated with gene silencing. ${ }^{37}$ A distinct subset of CRCs shows a phenotype characterized by widespread methylation: the CpG island methylator phenotype (CIMP). CIMP is thought to be an early event in tumorigenesis ${ }^{10}$ and potentially links energy balance to CRC risk because obesity has been associated with inflammation ${ }^{38,39}$ and inflammation has been associated with methylation. ${ }^{40}$ With respect to the IGF pathway, promoter hypermethylation of growthinhibiting IGFBP genes has been described ${ }^{41-46}$ and could be unfavorable in relation to CRC.

\subsubsection{Genetic instability}

Strongly correlated with CIMP is microsatellite instability (MSI), which involves changes in short repeated DNA sequences ('microsatellites'). ${ }^{47}$ While the cause of CIMP is unknown and CIMP tumors without MSI have been associated with poor prognosis, MSI is caused by methylation of the DNA repair genes MLH1 and MGMT and is associated with good prognosis. ${ }^{37,47,48}$ Although CIMP and MSI are important, the majority of CRCs are characterized by chromosomal instability (CIN), which is associated with an abnormal chromosomal content (aneuploidy), possibly reflecting an increased rate at which (large parts of) chromosomes are gained or lost. ${ }^{49,50} \mathrm{CIN}$ has been understudied as compared to MSI and CIMP. 
Box 2. Indicators of energy balance in adult life and early life.

A positive energy balance results when caloric intake exceeds energy expenditure. The surplus of calories will be stored as fat. A negative energy balance and potential subsequent weight loss may result from caloric restriction and physical activity. For obvious ethical reasons, caloric restriction is difficult to study in humans, but it has long been recognized that caloric restriction convincingly lowers cancer risk in rodents. ${ }^{51}$

- The indicators of energy balance investigated in this thesis were:

Adult body mass index (BMI; weight in kilograms divided by height in metres squared)

Adult trouser/skirt size, used as a proxy measure for waist circumference

(Waist circumference is a measure for abdominal fatness, and may be a stronger risk factor for CRC than BMI. ${ }^{52,53}$ Waist circumference has been shown to be a predictor of visceral fat (fat between organs in the abdomen), which gives rise to an inflammatory state associated with insulin resistance. ${ }^{54}$ )

(Non-)occupational physical activity

$\mathrm{BMl}$ at age 20

Height

(Height has been shown a CRC risk factor independent of BMI and is thought to be a marker of earlier life exposures, such as exposure to growth factors.)

Early life energy restriction as measured through place of residence during the Hunger Winter (1944-45), place of residence during the War Years (1940-44) and employment status of an individual's father during the Economic Depression (1932-40)

Of note is that we did not investigate total energy intake as an indicator of energy balance, because underreporting of food intake is a limitation of self-reported food intake through a food-frequency questionnaire, and the error of self-reported food intake may vary by participant characteristics. ${ }^{55}$ The variation observed in the population therefore may not reflect the absolute number of calories consumed.

\subsection{Aim of this thesis}

The overall aim of this thesis was to shed light on the pathways linking energy balance to CRC risk, in order to explain part of the heterogeneity observed in sex- and subsite-specific associations. To achieve this, firstly, associations were studied between indicators of energy balance and the risk of CRC, colon cancer, proximal colon cancer, distal colon cancer and rectal cancer in men and women. The specific indicators of energy balance investigated in this thesis are presented in Box 2. We hypothesized that indicators of a positive energy balance increase CRC risk, whereas indicators of a negative energy balance decrease $\mathrm{CRC}$ risk.

Then, the IGF pathway was studied as a potential pathway linking indicators of energy balance to CRC risk. The molecular epidemiological approach taken was two-fold: 1) potential combined effects were studied of in- dicators of energy balance and genetic variants in genes in the IGF pathway and in closely related genes in relation to CRC endpoints; and 2) indicators of energy balance were studied in relation to the risk of CRC by IGFBP methylation status. We hypothesized that a diminished functioning of the IGF pathway, either through carrying unfavorable genetic variants or through methylation of IGFBP genes, renders individuals particularly susceptible to the influence of energy balance on the risk of developing a CRC.

Furthermore, indicators of energy balance were studied in relation to the risk of CRC by CIMP status. CIMP is thought to be reflective of a major disease pathway involving methylation in CRC. We hypothesized that CIMP may also render individuals particularly susceptible to the influence of energy balance on the risk of developing a CRC. 
Finally, because CIMP and its correlate microsatellite instability are thought to hold differential prognostic value, which may depend on the tumor's chromosomal instability status, we evaluated the interrelationships between these three major instability types and studied associations with CRC-related death in an additional chapter.

\subsection{Study framework}

All studies included in this thesis were conducted within the Netherlands Cohort Study (NLCS) on diet and cancer. The NLCS includes 120,852 men and women who were between 55-69 years old at baseline (1986). At baseline, all participants completed a selfadministered questionnaire on diet, lifestyle and other risk factors for cancer. ${ }^{56}$ Along with returning the questionnaire, participants were asked to return toenail clippings, which were used to determine selenium status, and turned out to be a long-term DNA source for the genotyping of germline genetic variants. ${ }^{57}$

The design of the NLCS entails that incident cancer cases are followed up for the whole cohort, while a subcohort is followed up to estimate the accumulated person-time at risk. This case-cohort approach allows for the calculation of so-called 'pseudo-rates' for cancer occurrence within categories of exposure (Figure 3). Since the followed up person-time at risk is a fraction of the total person-time at risk in the entire cohort, these rates are clearly overestimated, yet can be used to derive hazard ratios for cancer, under the assumption that the sampling fraction in different exposure categories is equal (Figure 3). ${ }^{32}$ This assumption is met when the subcohort is sampled independently of exposure, as has been done in the NLCS (the subcohort was sampled at random, immediately after baseline). In this thesis, follow-up data were used from two different periods. The period 19862003 - the maximum follow-up time available at the start of this research project - was used to study overall associations and to study combined effects of indicators of energy balance and genetic variants in IGF pathway genes and closely related genes. The period 1989-1993 was used to study molecular endpoints, because tumor material has been collected for the tumors that occurred during this period.

\section{Toenail and tumor DNA}

For the molecular epidemiological studies described in this thesis, toenail and tumor DNA were used. Toenail DNA was isolated from toenail clippings of subcohort members, CRC cases and other cancer cases during the course of this project (2009-2013). Toenail DNA proved a valid DNA source for use on the SEQUENOM ${ }^{\circledR}$ MassARRAY ${ }^{\circledR}$ platform in a pilot study. This platform allows highthroughput genotyping of a maximum of 40 SNPs at once using one multiplex assay. With respect to the choice of genotyping platform, the quality of toenail DNA had to be taken into consideration. Toenail DNA samples contained relatively short DNA fragments (mostly $<200$ base pairs) and showed more protein and organic contamination than the norm (as based on 21,900 samples). Furthermore, the mean DNA yield from $15 \mathrm{mg}$ of toenail clippings, which is the optimum amount for DNA isolation, was $4.7 \mu \mathrm{g}$ (standard deviation of 8.9). Pilot studies for the use of toenail DNA on other (larger) genotyping platforms are ongoing.

Tumor DNA of CRC cases that occurred in the period 1989-1993 was isolated from five 20 $\mu \mathrm{m}$ sections of the formalin-fixed, paraffinembedded tumor block. After deparaffination of the sections, tumor material was separated from normal epithelium using a larger section as reference. This $5 \mu \mathrm{m}$ section was stained with haematoxylin and eosin for histopathological examination. Although the amount of 
isolated DNA was sufficient, purification of samples was necessary for some specific analyses (e.g. the analysis of chromosomal instability). Tumor DNA, similar to toenail DNA, was fragmented (100-400 bp), which depended on the conditions of fixation and storage. Nevertheless, both types of DNA were invaluable for the molecular epidemiological studies described in this thesis.

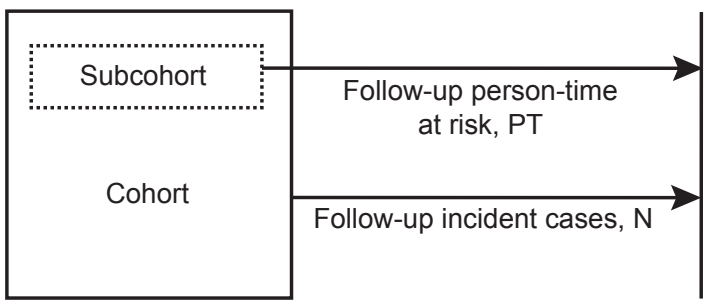

$$
H R=\frac{\left(N_{1} /\left(f * P T_{1}\right)\right)}{\left(N_{0} /\left(f * P T_{0}\right)\right)}=\frac{\left(N_{1} / P T_{1}\right)}{\left(N_{0} / P T_{0}\right)}
$$

Figure 3. Estimation of hazard ratios using the case-cohort design (Rothman and Greenland ${ }^{32}$ ).

Abbreviations: $H R$, hazard ratio; $N_{1}$ and $P T_{1}$ represent the number of incident cancer cases in the entire cohort and the accumulated person-time at risk in the subcohort, respectively, during a specific follow-up period in the exposed group; $N_{0}$ and $P T_{0}$ represent the number of incident cancer cases in the entire cohort and the accumulated person-time at risk in the subcohort, respectively, during a specific follow-up period in the unexposed group; $f$ represents the sampling fraction.

\subsection{Thesis outline}

In chapters 2 and 3 of this thesis, associations are described between indicators of energy balance and the subsite-specific CRC risk in men and women. In chapter 4 , we describe combined effects of indicators of energy balance and genetic variants in IGF pathway genes and closely related genes in relation to CRC endpoints. In chapters 5 and 6, we describe indicators of energy balance in relation to the risk of CRC by specific molecular characteristics, i.e. methylation status of IGFBP genes and CIMP status, respectively. In chapter 7 , we describe the prognostic value of specific tumor instability types in CRC. Finally, chapter 8 provides a perspective on the study of gene-environment interactions in CRC and a discussion of the main findings described in this thesis. 


\section{References}

1. International Agency for Research on Cancer (IARC). GLOBOCAN 2008.

http://globocan.iarc.fr/factsheet.asp. Accessed: December 30, 2012.

2. Van Wezel, T., Middeldorp, A., Wijnen, J. T. \& Morreau, $\mathrm{H}$. A review of the genetic background and tumour profiling in familial colorectal cancer. Mutagenesis 27, 239-45 (2012).

3. World Cancer Research Fund / American Institute for Cancer Research. Food, Nutrition, Physical Activity, and the Prevention of Cancer: a Global Perspective. Washington DC: AICR, 2007.

4. Liang, P. S., Chen, T. Y. \& Giovannucci, E. Cigarette smoking and colorectal cancer incidence and mortality: systematic review and meta-analysis. Int J Cancer 124, 2406-15 (2009).

5. Gong, J. et al. A pooled analysis of smoking and colorectal cancer: timing of exposure and interactions with environmental factors. Cancer Epidemiol Biomarkers Prev 21, 1974-85 (2012).

6. Koo, J. H. \& Leong, R. W. Sex differences in epidemiological, clinical and pathological characteristics of colorectal cancer. J Gastroenterol Hepatol 25, 33-42 (2010).

7. Li, F. Y. \& Lai, M. D. Colorectal cancer, one entity or three. J Zhejiang Univ Sci B 10, 219-29 (2009).

8. Iacopetta, B. Are there two sides to colorectal cancer? Int J Cancer 101, 403-8 (2002).

9. Jass, J. R. Classification of colorectal cancer based on correlation of clinical, morphological and molecular features. Histopathology 50, 113-30 (2007).

10. Issa, J. P. Colon cancer: it's CIN or CIMP. Clin Cancer Res 14, 5939-40 (2008).

11. Slattery, M. L. et al. Energy balance, insulinrelated genes and risk of colon and rectal cancer. Int J Cancer 115, 148-54 (2005).

12. McLannahan, H. \& Clifton, P. Challenging obesity: The science behind the issues. New York, NY: Oxford University Press, 2008.

13. Lund, E. K., Belshaw, N. J., Elliott, G. O. \& Johnson, I. T. Recent advances in understanding the role of diet and obesity in the development of colorectal cancer. Proc Nutr Soc 70, 194-204 (2011).

14. Sikalidis, A. K. \& Varamini, B. Roles of hormones and signaling molecules in describing the relationship between obesity and colon cancer. Pathol Oncol Res 17, 785-790 (2011).

15. Kaaks, R. \& Lukanova, A. Energy balance and cancer: the role of insulin and insulin-like growth factor-I. Proc Nutr Soc 60, 91-106 (2001).
16. Yu, H. \& Rohan, T. Role of the insulin-like growth factor family in cancer development and progression. J Natl Cancer Inst 92, 1472-89 (2000).

17. Durai, R., Yang, W., Gupta, S., Seifalian, A. M. \& Winslet, M. C. The role of the insulin-like growth factor system in colorectal cancer: review of current knowledge. Int J Colorectal Dis 20, 203-20 (2005).

18. Komninou, D., Ayonote, A., Richie, J. P. \& Rigas, $B$. Insulin resistance and its contribution to colon carcinogenesis. Exp Biol Med 228, 396-405 (2003).

19. Deng, L., Gui, Z., Zhao, L., Wang, J. \& Shen, L. Diabetes mellitus and the incidence of colorectal cancer: an updated systematic review and metaanalysis. Dig Dis Sci 57, 1576-1585 (2012).

20. Wild, C, Vineis, P \& Garte, S. Molecular Epidemiology of Chronic Diseases. Chichester, England: John Wiley \& Sons Ltd, 2008.

21. Ogino, S., Chan, A. T., Fuchs, C. S. \& Giovannucci, E. Molecular pathological epidemiology of colorectal neoplasia: an emerging transdisciplinary and interdisciplinary field. Gut 60, 397-411 (2010).

22. Attia, J. et al. How to use an article about genetic association: A: Background concepts. JAMA 301, 74-81 (2009).

23. Górecki, D. C., Beresewicz, M. \& Zabłocka, B. Neuroprotective effects of short peptides derived from the Insulin-like growth factor 1 . Neurochem Int 51, 451-458 (2007).

24. Shavlakadze, T., Winn, N., Rosenthal, N. \& Grounds, M. D. Reconciling data from transgenic mice that overexpress IGF-I specifically in skeletal muscle. Growth Horm IGF Res 15, 4-18 (2005).

25. dbSNP Home Page. At: http://www.ncbi.nlm. nih.gov. Accessed: January 6, 2013.

26. Ensembl Genome Browser. At: http://www. ensembl.org. Accessed: July 2, 2013.

27. Hunter, D. J. Lessons from genome-wide association studies for epidemiology. Epidemiology 23, 363-367 (2012).

28. Pritchard, J. K. \& Przeworski, M. Linkage disequilibrium in humans: models and data. Am J Hum Genet 69, 1-14 (2001).

29. Hrafnkelsson, B. et al. Evaluating differences in linkage disequilibrium between populations. Ann Hum Genet 74, 233-47

30. Yang, Q. \& Khoury, M. J. Evolving methods in genetic epidemiology. III. Gene-environment interaction in epidemiologic research. Epidemiol Rev 19, 33-43 (1997). 
31. Greenland, S. Interactions in epidemiology: relevance, identification, and estimation.

Epidemiology 20, 14-17 (2009).

32. Rothman, K. J. \& Greenland, S. Modern Epidemiology. Philadelphia, PA: Lippincott-Raven Publishers, 1998.

33. Khoury, M. J., Beaty, T. H. \& Cohen, B. H. Fundamentals of genetic epidemiology. New York, NY: Oxford University Press, 1993.

34. García-Closas, M. et al. NAT2 slow acetylation, GSTM1 null genotype, and risk of bladder cancer: results from the Spanish Bladder Cancer Study and meta-analyses. Lancet 366, 649-659 (2005).

35. Rothman, N. et al. Molecular epidemiology: principles and practices. IARC Scientific Publications, No. 163. Geneva, Switzerland: WHO press, 2011.

36. Baylin, S. B. et al. Abnormal patterns of DNA methylation in human neoplasia: potential consequences for tumor progression. Cancer Cells 3, 383-90 (1991).

37. Van Engeland, M., Derks, S., Smits, K. M., Meijer, G. A. \& Herman, J. G. Colorectal cancer epigenetics: complex simplicity. J Clin Oncol 29, 1382-91 (2011).

38. Dossus, L. \& Kaaks, R. Nutrition, metabolic factors and cancer risk. Best Pract Res Clin Endocrinol Metab 22, 551-571 (2008).

39. Monteiro, R. \& Azevedo, I. Chronic inflammation in obesity and the metabolic syndrome. Mediators Inflamm 2010, (2010).

40. Suzuki, H. et al. Roles and causes of abnormal DNA methylation in gastrointestinal cancers. Asian Pac J Cancer Prev 7, 177-185 (2006).

41. Ibanez de Caceres, I. et al. Identification of novel target genes by an epigenetic reactivation screen of renal cancer. Cancer Res 66, 5021-5028 (2006).

42. Sato, H. et al. Growth regulation via insulin-like growth factor binding protein- 4 and -2 in association with mutant K-ras in lung epithelia. Am J Pathol 169, 1550-1566 (2006).

43. Lin, J. et al. Methylation patterns of IGFBP7 in colon cancer cell lines are associated with levels of gene expression. J Pathol 212, 83-90 (2007).

44. Ye, F. et al. Decreased expression of insulin-like growth factor binding protein 7 in human colorectal carcinoma is related to DNA methylation. J Cancer Res Clin Oncol 133, 305-314 (2007).
45. Vizioli, M. G. et al. IGFBP7: an oncosuppressor gene in thyroid carcinogenesis. Oncogene 29, 3835-3844 (2010).

46. Chen, Y. et al. IGFBP7 is a p53 target gene inactivated in human lung cancer by DNA hypermethylation. Lung Cancer 73, 38-44 (2011).

47. Boland, C. R. \& Goel, A. Microsatellite instability in colorectal cancer. Gastroenterology 138, 20732087.e3 (2010).

48. Ogino, S. \& Goel, A. Molecular classification and correlates in colorectal cancer. J Mol Diagn 10, 1327 (2008).

49. Rajagopalan, H. \& Lengauer, C. CIN-ful cancers. Cancer Chemother Pharmacol 54 Suppl 1, S65-68 (2004).

50. Pino, M. S. \& Chung, D. C. The chromosomal instability pathway in colon cancer. Gastroenterology 138, 2059-2072 (2010).

51. Kritchevsky, D. Colorectal cancer: the role of dietary fat and caloric restriction. Mutat Res 290, 6370 (1993).

52. Moore, L. L. et al. BMI and waist circumference as predictors of lifetime colon cancer risk in Framingham Study adults. Int J Obes Relat Metab Disord 28, 559-67 (2004).

53. Wang, Y. et al. A prospective study of waist circumference and body mass index in relation to colorectal cancer incidence. Cancer Causes Control 19, 783-92 (2008).

54. Doyle, S. L., Donohoe, C. L., Lysaght, J. \& Reynolds, J. V. Visceral obesity, metabolic syndrome, insulin resistance and cancer. Proc Nutr Soc 71, 181-9 (2011).

55. Horner, N. K. et al. Participant characteristics associated with errors in self-reported energy intake from the Women's Health Initiative foodfrequency questionnaire. Am J Clin Nutr 76, 76673 (2002).

56. Van den Brandt, P. A. et al. A large-scale prospective cohort study on diet and cancer in The Netherlands. J Clin Epidemiol 43, 285-95 (1990).

57. Van Breda, S. G. et al. Toenails: an easily accessible and long-term stable source of DNA for genetic analyses in large-scale epidemiological studies. Clin Chem 53, 1168-70 (2007). 


\section{Body size and colorectal cancer risk after 16.3 years of follow-up: an analysis from the Netherlands Cohort Study}

Laura AE Hughes, Colinda CJM Simons, Piet A van den Brandt, R Alexandra Goldbohm, Manon van Engeland and Matty P Weijenberg

Am J Epidemiol. 2011 Nov 15;174(10):1127-39. 


\section{-Abstract}

Background: A large body size may differentially influence the risk of colorectal cancer (CRC) by anatomic location.

Methods: The Netherlands Cohort Study includes 120,852 men and women aged 55-69 years who self-reported weight, height, and trouser/skirt size at baseline (1986), as well as weight at age 20 years. Derived variables included body mass index (BMI; weight $(\mathrm{kg}) /$ height $\left.(\mathrm{m})^{2}\right)$, BMI at age 20 years, and BMI change. After 16.3 years of follow-up, 2,316 CRC cases were available for case-cohort analysis.

Results/Conclusion: In men, the highest risk estimates were observed for body fat (per 5-unit increase in BMI: hazard ratio $(\mathrm{HR})=1.25,95 \%$ confidence interval $(\mathrm{CI})$ : 1.05, 1.46; highest quintile of trouser size versus lowest: $\mathrm{HR}=1.63,95 \% \mathrm{CI}: 1.17,2.29(P$-trend $=0.02))$ and appeared more closely associated with distal colon tumors (per 5-unit increase in BMI: HR $=1.42,95 \% \mathrm{CI}$ : 1.13, 1.79; highest quintile of trouser size versus the lowest: $\mathrm{HR}=2.56,95 \% \mathrm{CI}: 1.55,4.24(P-$ trend $<0.01)$ ) than with proximal colon or rectal tumors. In women, body fat was not associated with CRC risk unless it was considered simultaneously with physical activity; a large trouser/skirt size and a low level of physical activity increased the risk for all subtypes. Height was associated with the risk of CRC, especially distal colon tumors, in women only (highest quintile versus lowest: $\mathrm{HR}=1.53,95 \% \mathrm{CI}: 1.03,2.27$; $P$-trend $=0.05)$. 


\subsection{Introduction}

There is convincing evidence that a large body size increases the risk of colorectal cancer (CRC). ${ }^{1}$ CRC risk has traditionally been studied according to anatomic location in the colon or rectum. However, several anatomic, embryologic, and physiologic differences exist between subanatomic locations in the colorectal tract, and therefore arising tumors may have different etiologic pathways. ${ }^{2-5}$ Thus, it may be more rational to consider CRC risk at three different locations: the proximal colon, distal colon, and rectum. Case-control and prospective cohort data suggest that in both men and women, body mass index (BMI) is more strongly associated with tumors of the distal colon than with tumors of the proximal colon. ${ }^{6-13}$ Several studies have suggested that waist circumference, as a proxy measure for abdominal obesity, may be a better indicator of CRC risk than BMI ${ }^{14,15}$ and that adult attained height as a proxy measure for early-life nutritional and socioeconomic exposures is also a convincing risk factor for CRC. ${ }^{1}$ How other indicators of body size, such as waist circumference and height, might influence CRC risk at different tumor subsites is less clear.

At present, one can only hypothesize as to how indicators of body size may influence subsite-specific pathways of colorectal carcinogenesis. Approximately $85 \%$ of tumors are thought to develop via the traditional adenoma-carcinoma pathway, characterized by mutations in the KRAS oncogene and the APC tumor suppressor gene, as well as chromosomal instability. ${ }^{16}$ Such tumors are most frequently observed in the distal colon. ${ }^{3}$ The other $15 \%$ of tumors are thought to arise via the serrated neoplasia pathway, characterized in part by a high degree of epigenetic instability and microsatellite instability. ${ }^{16}$ Such tumors are most frequently observed in the proximal colon. ${ }^{17-19}$ It has been reported that adult BMI and indicators of abdominal obesity are associated more strongly with tumors that are not characterized by epigenetic instability. ${ }^{20-22}$ This suggests that adult body fat rather influences CRC risk via chromosomal instability and may explain the stronger associations observed between body fat and distal tumors.

Furthermore, timing of exposure may be important for defining pathways. It has been observed that a high BMI in early adulthood and a large BMI change from early adulthood to later adulthood are associated with a higher CRC risk; ${ }^{13}$ that a high BMI at age 20 years is associated with a greater risk of having a tumor characterized by epigenetic instability; ${ }^{21}$ and that taller persons have a higher risk of tumors characterized by epigenetic instability. ${ }^{21}$

Here, we used data from a prospective study, the Netherlands Cohort Study (NLCS), to investigate the association between multiple indicators of body size and the sex-specific risk of CRC, as well as the associations between these indicators and the sex-specific CRC risk at specific tumor subsites, including the proximal colon, distal colon, rectosigmoid junction, and rectum. We considered BMI and trouser/skirt size as adult indicators of body fat, and we also considered adult attained height, BMI at age 20 years, and BMI change when assessing risk in order to study whether timing of exposure was associated with differential risk. We hypothesized that body fat would be associated more with tumors of the distal colon, whereas height might be associated more with proximal colon tumors.

\subsection{Methods}

\subsubsection{Study population and design}

The NLCS includes 58,279 men and 62,573 women aged 55-69 years at baseline (1986) who completed a self-administered question- 
naire involving 150 food items, as well as questions on lifestyle and health. Municipal registries from throughout the Netherlands were used to constitute an efficient sampling frame. ${ }^{23}$ The NLCS uses a case-cohort approach for data processing and analysis; case subjects were derived from the entire cohort, and the number of person-years at risk for the entire cohort was estimated from a subcohort of 5,000 persons who were randomly sampled from the full cohort at baseline. All subcohort members who reported prevalent cancer (excluding skin cancer) at baseline were excluded from the analyses, leaving 4,654 participants. Further details of the NLCS design have been published elsewhere. ${ }^{23-25}$

Incident CRC cases were identified by annual record linkage to 9 regional cancer registries and a national pathology database (PALGA). ${ }^{26}$ The completeness of cancer follow-up in the NLCS is almost $100 \% .{ }^{27}$ CRCs were classified by anatomic location as proximal colon (International Classification of Diseases for Oncology, First Edition, codes 153.0, 153.1, 153.4, 153.5, and 153.6), distal colon (codes 153.2, 153.3, and 153.7), rectosigmoid (code 154.0), or rectal (code 154.1) tumors. Persons with incomplete or inconsistent baseline questionnaires were excluded. After additional exclusion of persons who had missing data on variables adjusted for in the multivariate analysis, 3,197 subcohort members and 2,316 CRC cases remained. Among men, the numbers of tumors by location were 327 proximal colon, 427 distal colon, 33 unspecified colon, 125 rectosigmoid junction, and 299 rectum; for women, the numbers were 459 proximal colon, 327 distal colon, 27 unspecified colon, 87 rectosigmoid, and 205 rectum.
2.2.2 Assessment of anthropometric variables, diet, and physical activity

Height (cm), body weight $(\mathrm{kg})$, and body weight at age 20 years $(\mathrm{kg})$ were self-reported on the baseline questionnaire. BMI was calculated as weight $(\mathrm{kg}) /$ height $(\mathrm{m})^{2}$. Participants were also asked to report their lower-body clothing (trouser or skirt) size from their clothing label (in Dutch sizes). Trouser/skirt size has been shown to be an adequate proxy measure for waist circumference when predicting cancer risk in the NLCS. ${ }^{28}$

Both occupational physical activity in the longest-held job and baseline nonoccupational physical activity were used to assess risk, depending on sex. ${ }^{29}$ For analyses of men, occupational physical activity was used. Participants were asked to report job title(s) and job duration(s) on the baseline questionnaire. Assessment of physical activity at work was based on the job held for the longest amount of time. Total occupational energy expenditure was based on a rating system developed by Hettinger et al. ${ }^{30}$ Men were classified into 3 energy expenditure groups: $<8,8-12$, and $>12 \mathrm{~kJ} / \mathrm{min}$. For analyses with women, non-occupational physical activity was used to assess risk, because most women of this generation had not held a job or had worked for only a short period of time. It was calculated by totalling the number of minutes per day spent cycling/walking to work, going to shops, and walking the dog and the number of hours spent per week engaging in gardening/odd jobs, recreational cycling/walking, and sports/exercise, as reported on the baseline questionnaire. Women were classified as having a low $(\leq 30$ $\mathrm{min} /$ day), intermediate (>30-90 min/day), or high (>90 min/day) level of physical activity.

2.2.3 Statistical analysis

Data were analyzed with Stata, version 10 (StataCorp LP, College Station, Texas), sepa- 
rately for men and women. Cox proportional hazards analysis using the case-cohort approach was used to estimate hazard ratios and 95\% confidence intervals for the association between total CRC risk and BMI (per 5-unit increase, in sex-specific quintiles), BMI at age 20 years (per 5 -unit increase, in sex-specific quintiles), BMI change $(<0,0-<4,4-<8$, or $\geq 8$ units; categories were based on a previous NLCS publication ${ }^{31}$ ), trouser/skirt size (per 2unit size increase, in 5 sex-specific categories), and height (per 5-cm increase, in sex-specific quintiles). To test for a linear trend across categories, we used the median anthropometric variable within quintiles/categories as a continuous variable. Statistical significance was defined as a $P$-value $<0.05$ for two-sided testing.

Multivariate models. We adjusted for potential confounders selected a priori from the literature and those that introduced more than a $10 \%$ change in hazard ratios. Selected a priori were age, family history of CRC (yes/no), smoking status (never smoker, ex-smoker, or current smoker), socioeconomic status (educational level), total energy intake (kcal/day), alcohol intake $(0,0.1-4,5-14,15-29$, or $\geq 30$ $\mathrm{g} /$ day), recreational physical activity $(\leq 30$, $>30-90$, or $>90 \mathrm{~min} /$ day) for women, and occupational physical activity at the longestheld job $(<8,8-12$, or $>12 \mathrm{~kJ} / \mathrm{min})$ for men. No variables introduced more than a $10 \%$ change in the hazard ratios. BMI change was additionally adjusted for BMI at age 20 years, and height was additionally adjusted for body weight. ${ }^{31}$ We also considered a model for women that included additional adjustment for oral contraceptive use (ever, never), duration of oral contraceptive use (years), use of hormone replacement therapy (ever, never), and duration of hormone replacement therapy (years). This extra adjustment did not influence effect estimates, and results are not presented.
Interaction with physical activity. Evidence suggests that among persons with lower physical activity, BMI becomes a more important indicator of colon cancer risk ${ }^{9}$ and that underlying population levels of physical activity can impair or enhance the ability to identify colon cancer associations with other risk factors. ${ }^{32}$ We created a two-way interaction between trouser/skirt size and physical activity. Categories of physical activity were based on occupational physical activity (men) and selfreported recreational physical activity (women), and trouser/skirt size dichotomized on the basis of sex-specific median size. We used trouser/skirt size because evidence suggests that fat distribution is more important than body weight or BMI for CRC risk, especially in women. ${ }^{14}$ We also considered this interaction for colonic and rectal tumors separately.

For all analyses, the proportional hazards assumption was tested using scaled Schoenfeld residuals and visual inspection of the -log-logtransformed hazard curves. Furthermore, we repeated the analyses according to duration of follow-up by splitting the follow-up time at 8 years ( $<8$ years versus $\geq 8$ years). To account for the additional variance introduced by sampling the subcohort from the entire cohort, we estimated standard errors using the robust option.

Tests for heterogeneity. We conducted tests for heterogeneity to evaluate differences between sublocalizations of tumors (e.g. proximal colon versus distal colon), using the competing-risks procedure in Stata. However, the standard error for the difference in the log hazard ratios from this procedure assumes independence of both estimated hazard ratios, which would overestimate that standard error and thus overestimate the $P$-value for their difference. Therefore, we estimated these $P$-values and the associated confidence intervals on the basis of a bootstrapping method that was developed for the case-cohort design, as described 
previously. ${ }^{33}$ Each bootstrap analysis was based on 1,000 replications.

\subsection{Results}

\subsubsection{Descriptive characteristics}

For both men and women, a higher trouser/skirt size corresponded with an increasing BMI, and total energy intake was highest in the third quintile of BMI. Furthermore, the greatest proportion of persons with a university-level education was observed in the lowest quintile of BMI, as was the greatest proportion of current smokers. In men, levels of occupational physical activity were relatively similar across quintiles of BMI, whereas in women, higher levels of recreational physical activity were reported by persons in the lowest quintile of BMI (Tables 1 and 2).

\subsubsection{Associations with overall CRC in men}

Age-adjusted results are not presented, since they were comparable with multivariate results. Associations between body size and CRC risk in men are presented in Table 3. With respect to body fat, BMI modeled per 5unit increase was associated with total CRC (hazard ratio $(\mathrm{HR})=1.25,95 \%$ confidence interval (CI): 1.05, 1.46). When BMI was modeled in quintiles, associations did not reach statistical significance. Stronger associations were observed with respect to trouser size; a statistically significant association was observed when comparing persons with the largest trouser size to those with the smallest size $(\mathrm{HR}=1.63,95 \% \mathrm{CI}: 1.17,2.29 ; P$-trend $=$ 0.02). With respect to BMI at age 20 years, BMI change, and height, associations with overall CRC did not reach statistical significance.

\subsubsection{Associations by tumor subsite in men}

All indicators of body fat appeared to be most strongly associated with tumors of the distal colon. Increasing BMI (per 5 units) was asso- ciated with both distal ( $\mathrm{HR}=1.42,95 \% \mathrm{CI}$ : $1.13,1.79)$ and rectosigmoid $(\mathrm{HR}=1.49,95 \%$ CI: $1.05,2.11)$ tumors. A statistically significant trend was observed over quintiles of BMI for these two subsites $(P=0.05)$. BMI at age 20 years was associated only with tumors of the distal colon (5-unit increase: $\mathrm{HR}=1.09,95 \%$ CI: 1.00, 1.19; highest quintile versus lowest: $\mathrm{HR}=1.47,95 \%$ CI: 1.03, 2.08; P-trend $=0.05$ ). With respect to a positive BMI change, there was a suggested dose-response association for tumors of the distal colon $(P$-trend $=0.05)$. The strongest association was observed with respect to trouser size and the distal colon (largest size versus smallest: $\mathrm{HR}=2.56,95 \% \mathrm{CI}$ : $1.55,4.24 ; P$-trend < 0.01$)$. With respect to height, an inverse association was observed for rectosigmoid tumors (5-cm increase: $\mathrm{HR}=$ 0.77, 95\% CI: 0.65, 0.93; highest quintile versus lowest: $\mathrm{HR}=0.38,95 \%$ CI: 0.17, 0.83; $P$-trend $=$ 0.01).

The strengths of the associations did not differ when data were split according to duration of follow-up ( $<8$ years versus $\geq 8$ years). Tests for heterogeneity between tumor subtypes were not statistically significant for any risk factors considered.

\subsubsection{Associations with overall CRC in women}

In women, there were no statistically significant associations between BMI, BMI at age 20 years, BMI change, or trouser/skirt size and overall CRC risk (Table 4).

Height, modeled per 5-cm increase, was associated with a higher risk of total CRC $(\mathrm{HR}=$ 1.09, 95\% CI: 1.01, 1.17). There was also an association when the highest quintile was compared with the lowest quintile $(\mathrm{HR}=1.32$, 95\% CI: 1.03, 1.71; P-trend $=0.05)$.

2.3.5 Associations by tumor subsite in women

Associations by subsite in women were not as clear as those in men. With respect to body 
Table 1. Baseline characteristics of male subcohort members of the NLCS according to quintiles of body mass index, Netherlands Cohort Study (1986)*

\begin{tabular}{|c|c|c|c|c|c|}
\hline & \multicolumn{5}{|c|}{ Quintile of body mass index (BMI) $\left(\mathrm{kg} / \mathrm{m}^{2}\right) \dagger$} \\
\hline & 1 (lowest) & 2 & 3 & 4 & 5 (highest) \\
\hline $\mathrm{N}$ & 289 & 282 & 280 & 265 & 249 \\
\hline Age at baselineł & $61.0(4.3)$ & $61.0(4.1)$ & $60.9(4.2)$ & $61.0(4.2)$ & $61.2(4.2)$ \\
\hline Total energy intake (kcal/day) & $2,115(445)$ & $2,194(485)$ & $2,202(480)$ & $2,147(505)$ & $2,172(523)$ \\
\hline Weight $(\mathrm{kg})$ & $67.9(6.1)$ & $74.2(5.6)$ & $77.4(5.4)$ & $81.4(6.3)$ & $88.9(8.9)$ \\
\hline Height $(\mathrm{cm})$ & $177.5(6.7)$ & $177.1(6.6)$ & $176.7(6.0)$ & $176.7(6.5)$ & $176.0(6.5)$ \\
\hline BMI $\left(\mathrm{kg} / \mathrm{m}^{2}\right) \S$ & 22.0 & 23.7 & 24.9 & 26.1 & 28.4 \\
\hline BMI at age $20\left(\mathrm{~kg} / \mathrm{m}^{2}\right) \S$ & 18.9 & 20.5 & 21.7 & 22.9 & 24.7 \\
\hline Trouser size & $50(2)$ & $50(2)$ & $52(2)$ & $52(2)$ & $54(2)$ \\
\hline \multicolumn{6}{|l|}{ Occupational physical activity (\%) } \\
\hline$<8 \mathrm{kj} / \mathrm{min}$ & 63 & 61 & 62 & 60 & 60 \\
\hline $8-12$ & 26 & 25 & 24 & 26 & 26 \\
\hline$>12$ & 11 & 13 & 14 & 14 & 14 \\
\hline \multicolumn{6}{|l|}{ Level of education (\%) } \\
\hline Primary school & 18 & 19 & 23 & 27 & 29 \\
\hline Junior/senior high school & 18 & 19 & 21 & 22 & 21 \\
\hline Higher vocational school & 39 & 40 & 36 & 33 & 35 \\
\hline University & 24 & 23 & 20 & 18 & 15 \\
\hline \multicolumn{6}{|l|}{ Smoking status (\%) } \\
\hline Never smoker & 12 & 11 & 11 & 12 & 14 \\
\hline Ex-smoker & 47 & 54 & 57 & 59 & 57 \\
\hline Current smoker & 41 & 35 & 32 & 30 & 29 \\
\hline \multicolumn{6}{|l|}{ Alcohol intake (\%) } \\
\hline $0 \mathrm{~g} /$ day & 20 & 11 & 13 & 11 & 10 \\
\hline $0.1-4$ & 22 & 17 & 19 & 24 & 21 \\
\hline $5-14$ & 24 & 30 & 29 & 29 & 25 \\
\hline $15-29$ & 21 & 26 & 23 & 22 & 28 \\
\hline$\geq 30$ & 14 & 16 & 17 & 15 & 16 \\
\hline \multicolumn{6}{|l|}{ Family history of CRC (\%) } \\
\hline No & 96 & 95 & 93 & 96 & 95 \\
\hline Yes & 4 & 5 & 7 & 4 & 5 \\
\hline
\end{tabular}

Abbreviations: BMI, body mass index; SD, standard deviation

* Subcohort members who did not have missing data for the given exposure and any of the considered baseline characteristics.

† Range of BMI $\left(\mathrm{kg} / \mathrm{m}^{2}\right)$ : quintile 1, 16.1-23.0; quintile 2, 23.1-24.3; quintile 3, 24.3-25.4; quintile 4, 25.4-27.0; and quintile 5, 27.1-39.6.

$\ddagger$ Presented as mean (SD) or \% where indicated.

$\S$ Median BMI. 
Table 2. Baseline characteristics of female subcohort members of the NLCS according to quintiles of body mass index, Netherlands Cohort Study (1986)*

\begin{tabular}{|c|c|c|c|c|c|}
\hline & \multicolumn{5}{|c|}{ Quintile of body mass index (BMI) $\left(\mathrm{kg} / \mathrm{m}^{2}\right) \dagger$} \\
\hline & 1 (lowest) & 2 & 3 & 4 & 5 (highest) \\
\hline $\mathrm{N}$ & 359 & 373 & 369 & 373 & 358 \\
\hline Age at baseline $\ddagger$ & $61.2(4.5)$ & $61.0(4.3)$ & $61.1(4.2)$ & $61.6(4.2)$ & $61.4(4.1)$ \\
\hline Total energy intake (kcal/day) & $1,719(412)$ & $1,710(401)$ & $1,725(420)$ & $1,655(350)$ & $1,630(372)$ \\
\hline Weight $(\mathrm{kg})$ & $57.4(5.6)$ & $63.6(4.6)$ & $67.8(4.9)$ & $71.5(5.6)$ & $81.7(8.5)$ \\
\hline Height $(\mathrm{cm})$ & $166.2(6.1)$ & $165.6(5.9)$ & $165.9(5.9)$ & $164.4(6.2)$ & $163.9(5.9)$ \\
\hline BMI $\left(\mathrm{kg} / \mathrm{m}^{2}\right) \S$ & 21.3 & 23.4 & 24.7 & 26.3 & 29.4 \\
\hline BMI at age $20\left(\mathrm{~kg} / \mathrm{m}^{2}\right) \S$ & 18.0 & 20.0 & 21.3 & 22.6 & 24.6 \\
\hline Trouser size & $40(2)$ & $42(2)$ & $43(2)$ & $44(2)$ & $46(2)$ \\
\hline \multicolumn{6}{|l|}{ Recreational physical activity (\%) } \\
\hline$\leq 30 \mathrm{~min} /$ day & 20 & 19 & 23 & 25 & 25 \\
\hline$>30-60$ & 30 & 32 & 32 & 33 & 33 \\
\hline$>60-90$ & 25 & 26 & 23 & 21 & 21 \\
\hline$>90$ & 25 & 23 & 23 & 22 & 22 \\
\hline \multicolumn{6}{|l|}{ Level of education (\%) } \\
\hline Primary school & 26 & 27 & 31 & 38 & 39 \\
\hline Junior/senior high school & 17 & 24 & 26 & 25 & 28 \\
\hline Higher vocational school & 43 & 38 & 36 & 29 & 29 \\
\hline University & 13 & 12 & 7 & 8 & 4 \\
\hline \multicolumn{6}{|l|}{ Smoking status (\%) } \\
\hline Never smoker & 49 & 58 & 54 & 65 & 63 \\
\hline Ex-smoker & 24 & 18 & 25 & 21 & 19 \\
\hline Current smoker & 27 & 24 & 21 & 14 & 18 \\
\hline \multicolumn{6}{|l|}{ Alcohol intake (\%) } \\
\hline $0 \mathrm{~g} /$ day & 28 & 31 & 32 & 33 & 37 \\
\hline $0.1-4$ & 33 & 35 & 37 & 39 & 38 \\
\hline $5-14$ & 22 & 20 & 18 & 18 & 16 \\
\hline $15-29$ & 12 & 11 & 9 & 8 & 8 \\
\hline$\geq 30$ & 6 & 3 & 4 & 3 & 3 \\
\hline \multicolumn{6}{|l|}{ Family history of CRC (\%) } \\
\hline No & 94 & 94 & 93 & 95 & 94 \\
\hline Yes & 6 & 6 & 7 & 5 & 6 \\
\hline
\end{tabular}

Abbreviations: BMI, body mass index; SD, standard deviation

* Subcohort members who did not have missing data for the given exposure and any of the considered baseline characteristics.

† Range of BMI $\left(\mathrm{kg} / \mathrm{m}^{2}\right)$ : quintile 1, 15.4-22.2; quintile 2, 22.2-23.8; quintile 3, 23.9-25.4; quintile 4, 25.4-27.5; and quintile 5, 27.6-41.4.

$\ddagger$ Presented as mean (SD) or \% where indicated.

$\S$ Median BMI. 
fat, women with the largest trouser/skirt size had a borderline statistically significant risk of proximal colon tumors compared with those with the smallest size (HR $=1.46,95 \% \mathrm{CI}: 0.98$, $2.18)$, but there was no clear trend $(P=0.46)$.

There was a borderline statistically significant association between height and distal colon tumors (HR $=1.11,95 \% \mathrm{CI}: 0.99,1.24)$ and rectal tumors $(\mathrm{HR}=1.14,95 \% \mathrm{CI}: 0.98,1.32)$ when data were modeled per $5-\mathrm{cm}$ increase. Women in the highest quintile also had a statistically significant risk of distal tumors when compared with those in the lowest quintile $(\mathrm{HR}=1.53,95 \% \mathrm{CI}: 1.03,2.27$; $P$-trend $=0.05)$. This association was borderline statistically significant for rectal tumors ( $\mathrm{HR}=1.49,95 \%$ CI: $0.98,2.27$; $P$-trend $=0.06$ ).

When data were split at 8 years of follow-up, it appeared that associations for all anthropometric variables were attenuated over time (data not shown). Tests for heterogeneity between tumor subtypes were not statistically significant for any risk factors considered.

2.3.6 Interaction between trouser/skirt size and physical activity

Table 5 shows hazard ratios for the interaction between trouser size and occupational physical activity in men. Compared with the reference category of men with a small trouser size and a high level of physical activity, there were no statistically significant associations for total CRC, but men with a small trouser size and a low level of physical activity were at an increased risk of distal colon tumors (HR $=1.63,95 \%$ CI: 1.03, 2.56). A borderline statistically significant association was also observed for men with a large trouser size and all levels of physical activity with respect to tumors of the distal colon.

Compared with the reference category of women with a small trouser/skirt size and a high level of physical activity (Table 6), the greatest risk for each subtype considered appeared to be for persons with the highest trouser/skirt size and the lowest level of physical activity (for total CRC, $\mathrm{HR}=1.83$, 95\% CI: $1.28,2.63$; for proximal colon tumors, $\mathrm{HR}=1.70,95 \% \mathrm{CI}: 1.08,2.67$; for distal colon tumors, $\mathrm{HR}=1.95,95 \% \mathrm{CI}: 1.21,3.17$; for rectal tumors, $\mathrm{HR}=2.56,95 \% \mathrm{CI}: 1.36,4.79)$. The interaction between trouser/skirt size and physical activity was statistically significant only for the proximal colon $(P<0.05)$.

\subsection{Discussion}

We observed that in men, body fat, as indicated by BMI, BMI at age 20 years, BMI change, and trouser size, appeared to be more associated with distal tumors than with tumors at other anatomic subsites; however, associations with respect to height were less clear. Body size appeared to be unrelated to CRC outcomes in women, except for height, which was related positively to cancers of the distal colon and rectum. In general, our observations, especially in men, highlight the importance of examining CRC risks according to tumor subsite, because stronger associations were observed for individual subsites than with overall risk.

Strengths of this study include the prospective cohort design and the large population, in combination with sufficient follow-up time to allow precise analysis of CRC risk at different subsites. The NLCS has almost complete ascertainment of CRC cases, and follow-up of the subcohort is almost $100 \%$. Although measures of body size in this study were obtained by self-report, there are many examples in the literature showing that this method is a reasonably valid and reliable tool for assessing body weight and height in cohort studies. ${ }^{34-37}$ A weakness was the small number of rectosigmoid and rectal tumors and the subsequently limited statistical power. 


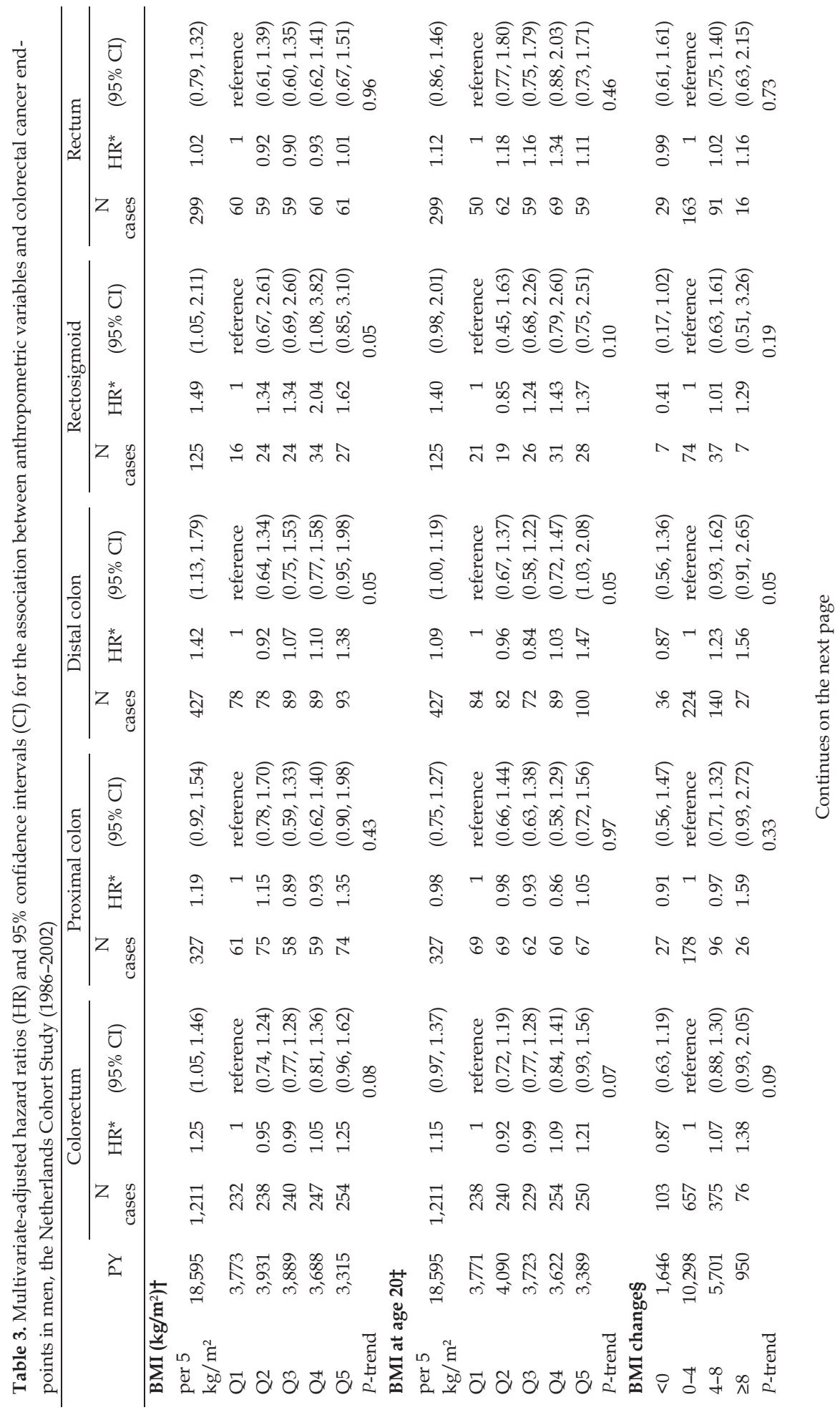




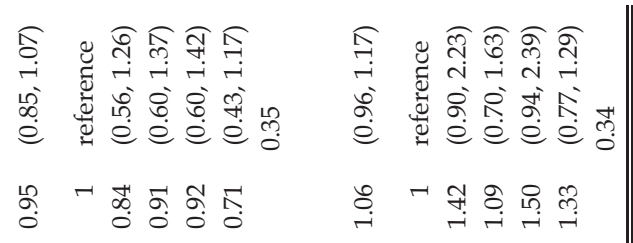

बे त ชु

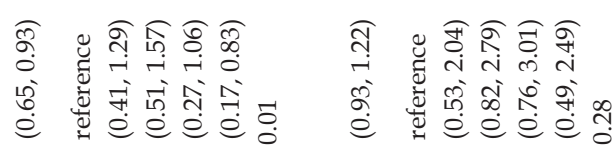

太ิ

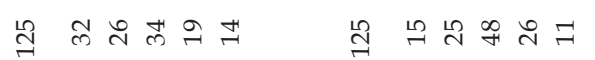

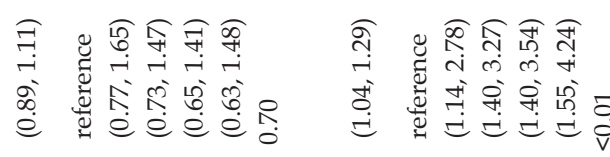

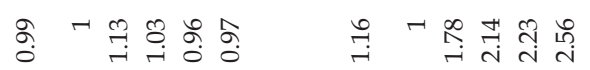

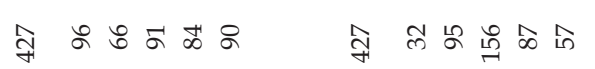

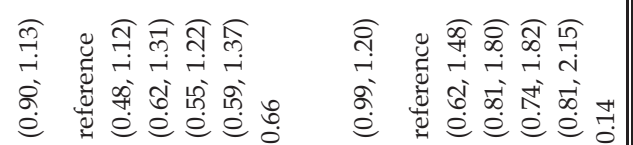

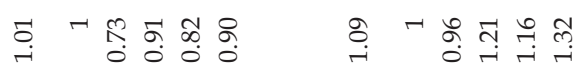

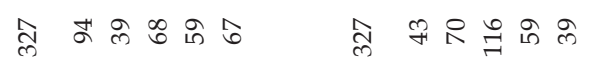

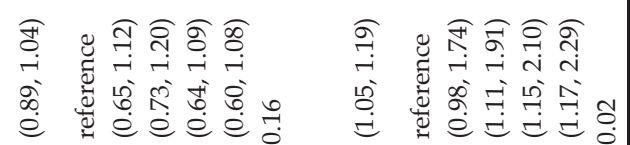

员

त्तु ले

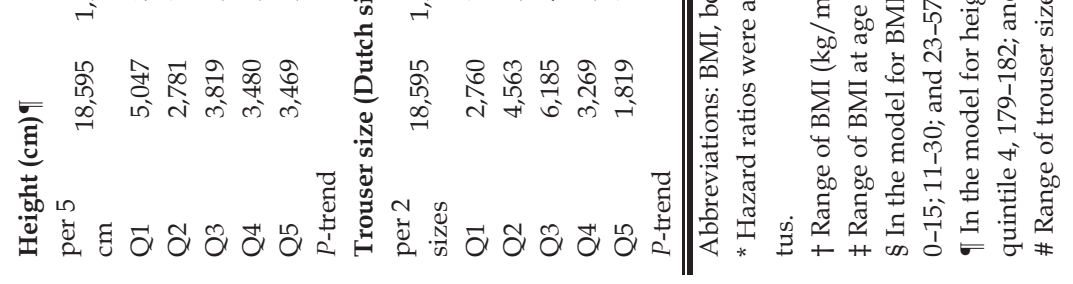




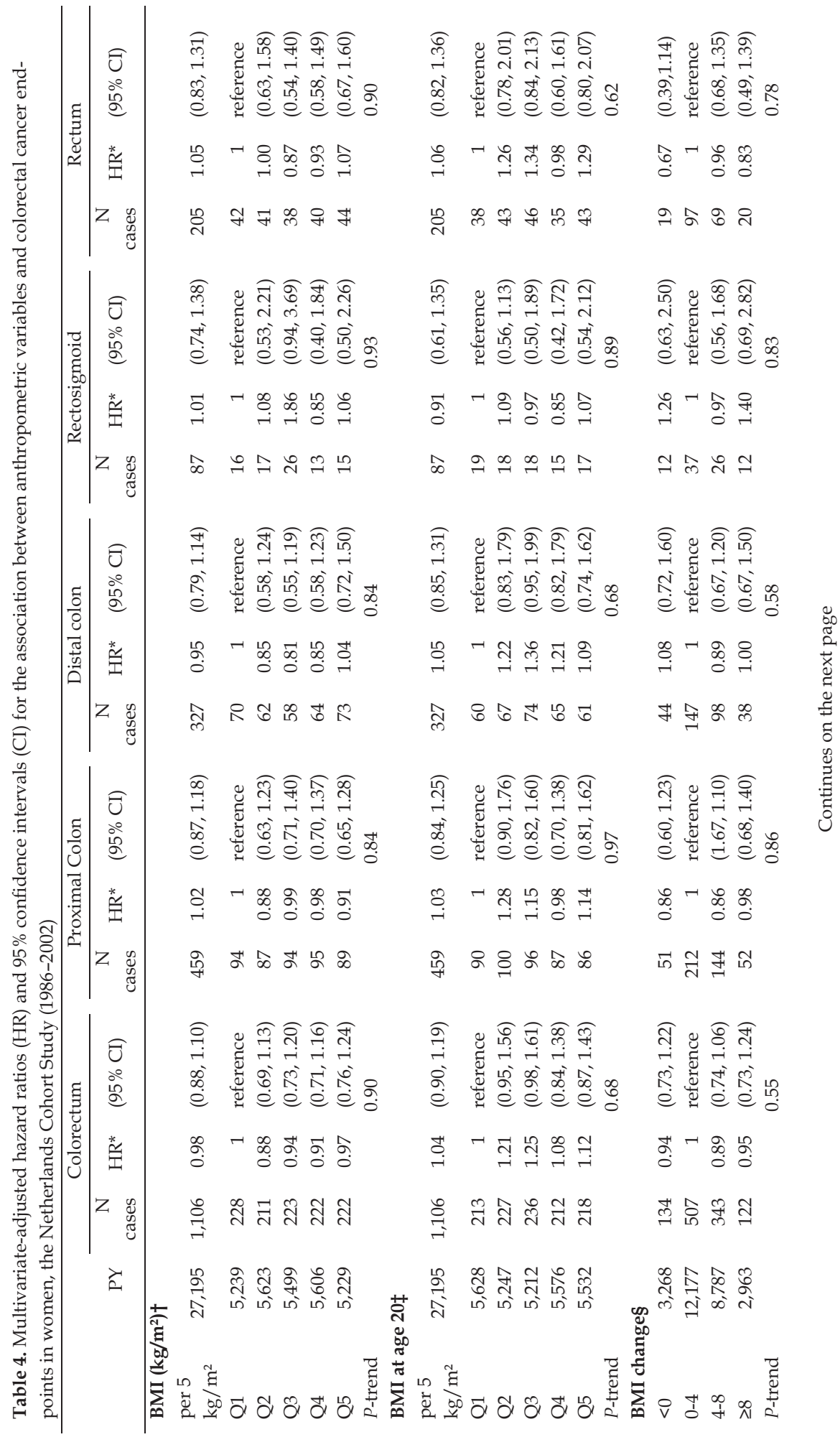




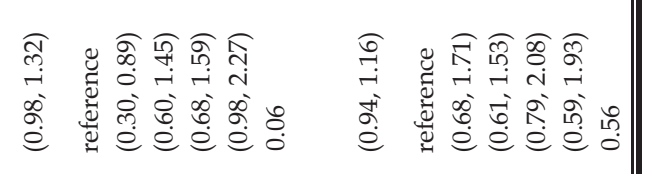

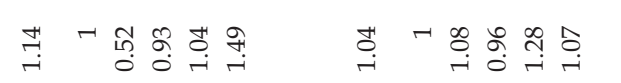

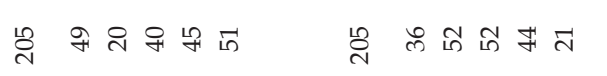

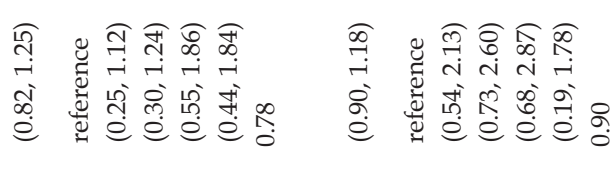

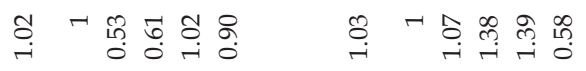
को त윰

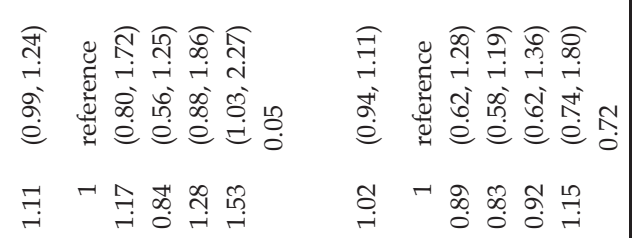

तิ

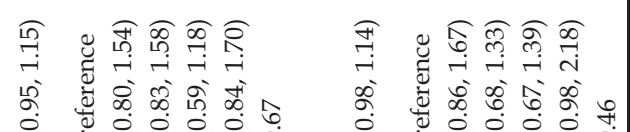

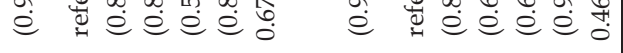

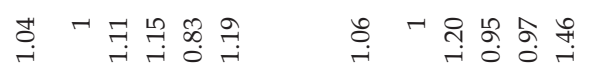

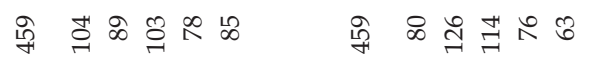

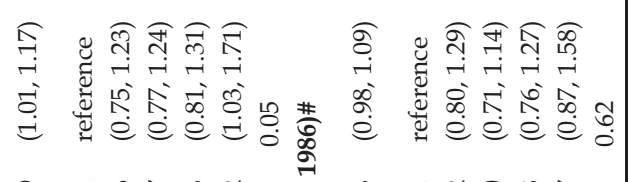

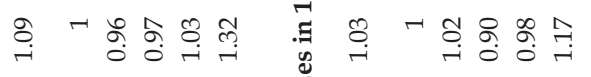

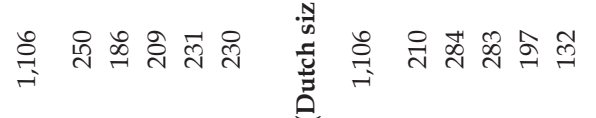

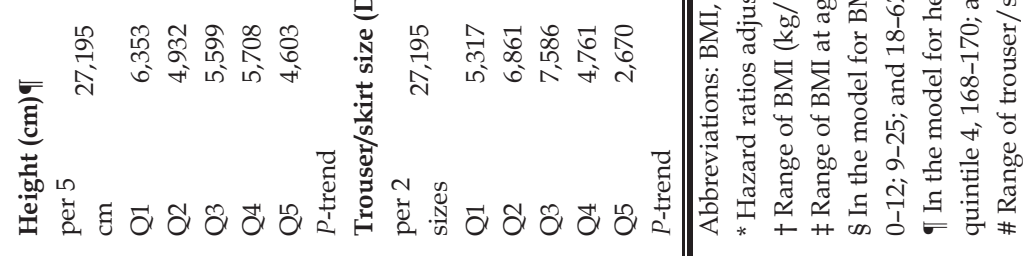




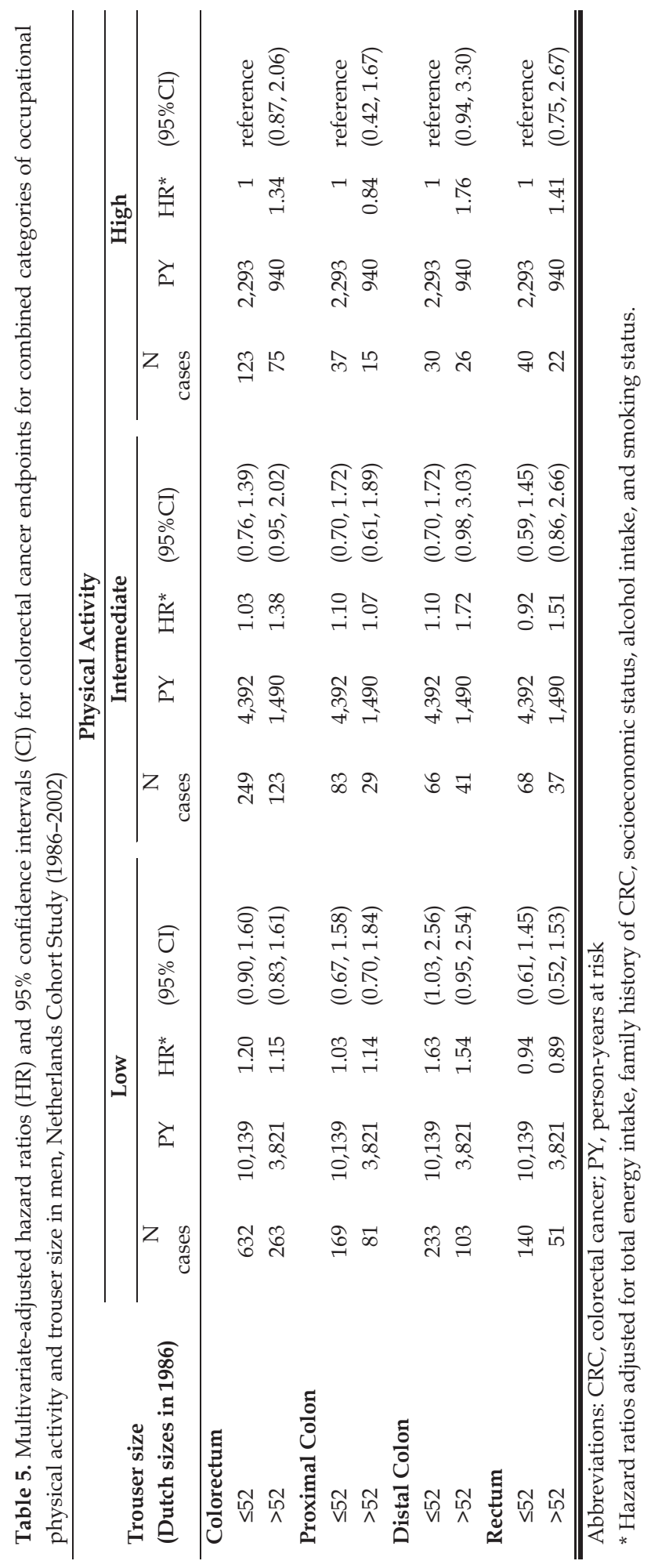




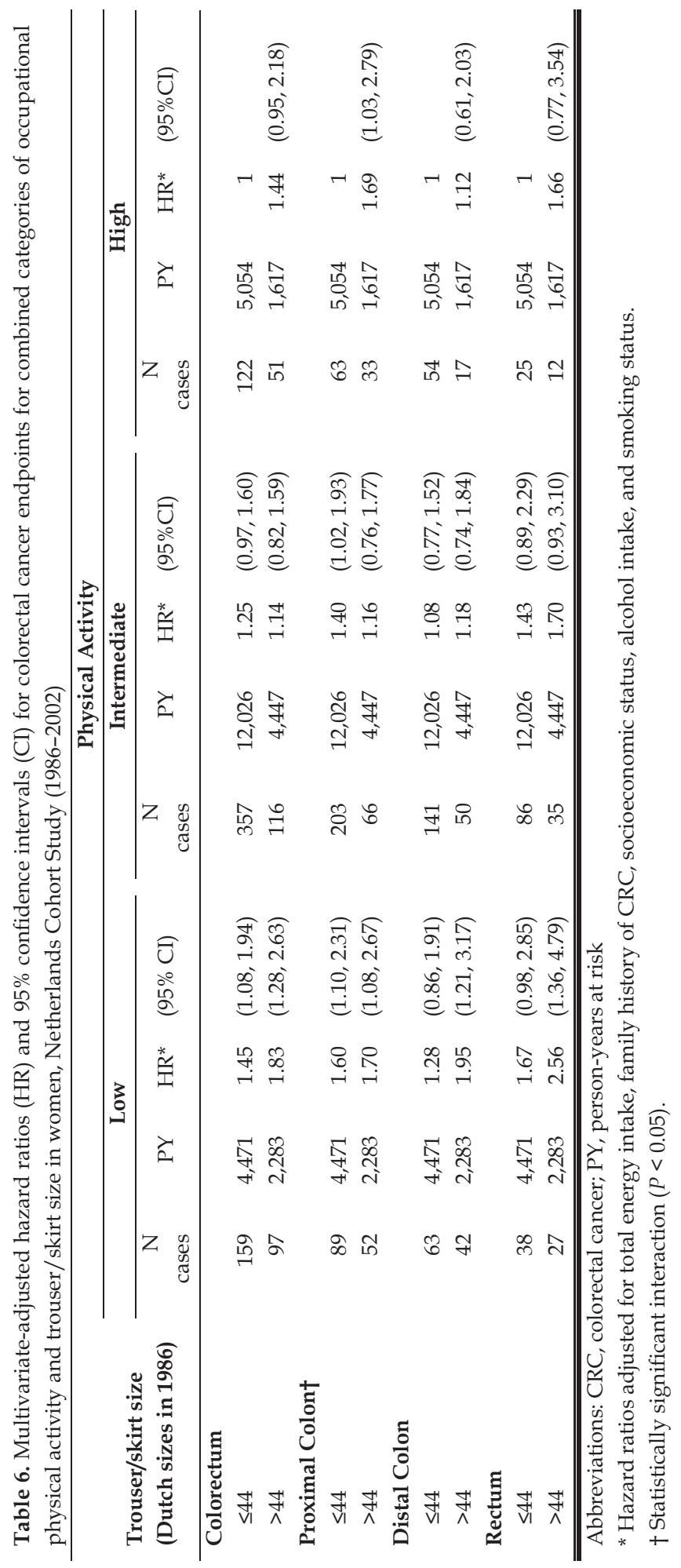


Investigators in several case-control studies have considered the association between BMI and site-specific tumors of the colon in men, and they have generally reported a stronger risk for tumors of the distal colon than for tumors of the proximal colon. ${ }^{6-10}$ This is supported by the results of a recent prospective cohort study carried out by Laake et al. ${ }^{11}$ In our study, we considered several indicators of body fat in addition to BMI, including BMI at age 20 years, BMI change, and trouser/skirt size as a proxy for waist circumference, and we consistently observed an elevated risk of distal colon tumors as compared with tumors at other subsites. Although we did not observe statistically significant heterogeneity between proximal and distal colon tumors as Laake et al. ${ }^{11} \mathrm{did}$, the bootstrapping method we used is quite conservative. The weaker associations that we observed with respect to associations between body fat and the rectum align with previous research, ${ }^{1}$ but we also observed a positive association between BMI and tumors of the rectosigmoid junction. To our knowledge, no other prospective study has considered the association between anthropometric measures and the rectosigmoid junction. This association is plausible, because the rectosigmoid junction and distal colon are anatomically linked. Our findings should be replicated in other prospective studies, but they support the hypothesis that the etiology of CRC tumors differs between anatomic subsites of the colorectal tract in men.

We did not observe clear associations between BMI, BMI at age 20 years, or BMI change and $\mathrm{CRC}$ risk in women, but our results suggest that women with a high trouser/skirt size are at increased risk of proximal colon tumors, although this association did not reach statistical significance. In contrast, positive associations between BMI and distal colon tumors have been reported in prospective cohort studies of Swedish ${ }^{12}$ and Norwegian ${ }^{11}$ women. In an American cohort, both BMI and waist circumference were associated with proximal and distal tumors. ${ }^{13}$ The proportion of obese women in the NLCS is small, and perhaps this prevented us from detecting associations of similar strength. It has also been suggested that the impact of a given risk factor along the length of the large bowel may differ according to the prevalence of other environmental factors and, thus, according to sex and country. ${ }^{2}$

Alternatively, it is plausible that a metabolic profile reflecting a combination of risk factors has a greater influence on cancer risk than do the individual effects of body size and obesity. ${ }^{38,39}$ Because evidence suggests that fat distribution is more important than body weight or BMI for CRC risk, especially in women, ${ }^{14}$ we investigated the interaction with physical activity using trouser/skirt size. Our observations for women were intriguing. As noted above, we did not observe statistically significant associations with respect to trouser/skirt size in our general analysis. However, when we considered the interaction between trouser/skirt size and physical activity, it appeared that women with a large trouser/skirt size and the lowest level of physical activity were at the greatest risk of CRC compared with women with a small trouser/skirt size and a high level of physical activity. This risk was highest for rectal tumors; however, the interaction was statistically significant only for tumors of the proximal colon. These findings require confirmation in other prospective studies, but they support case-control data.9,40 They also highlight that underlying population levels of physical activity can impair or enhance the ability to identify CRC associations with other risk factors, ${ }^{32}$ such as body fat.

Height is a reflection of earlier life exposures, such as childhood energy intake. ${ }^{41}$ In our study, height was associated with increased risk of CRC in women, which is in accordance with previous research. ${ }^{1,13,14,42,43}$ In a recent re- 
port, Oxentenko et al. ${ }^{13}$ suggested that risk is greater for tumors of the proximal colon, whereas our data suggest that risk is greater for tumors of the distal colon. However, in the former study, Oxentenko et al. did not report conducting tests for heterogeneity, and in our study, such tests did not reach statistical significance. We did not observe any association with respect to height and CRC risk in men, and we actually observed an inverse association for tumors of the rectosigmoid junction. We cannot explain this observation, and we suggest that it may be a chance finding. We have shown previously in the NLCS population that energy restriction during childhood is inversely related to later CRC risk; 44 therefore, while we have not observed strong associations between height and CRC in the present study, it is plausible that early-life nutritional factors may influence different carcinogenic pathways than later-life exposure to increased body fat. ${ }^{13}$

We can only speculate as to why body size and body fat in particular might differentially influence the etiology of colorectal tumors at different subsites. Obesity is associated with a chronic state of low-grade inflammation and thus increased circulatory levels of inflammatory markers like C-reactive protein, interleukin-6, and tumor necrosis factor a. ${ }^{45}$ These in turn may induce insulin resistance and hyperinsulinaemia. It is hypothesized that such conditions influence CRC risk; ${ }^{46-48}$ however, it remains unclear how they directly or indirectly influence specific tumor subsites. There are some distinct differences between the proximal colon and the distal colon, both anatomi- cally and genetically. ${ }^{3-5}$ The distal colon is associated more with physical/chemical stimuli and associated less with water absorption and electrolyte transport than the proximal colon. ${ }^{3}$ It has been shown that tumors characterized by chromosomal instability are more associated with tumors of the distal colon, whereas epigenetic changes like methylation are more associated with tumors of the proximal colon. ${ }^{49}$ Interestingly, recent studies have suggested that overweight and obesity do not appear to differentially influence CRC risk via epigenetic mechanisms and microsatellite instability. ${ }^{20,22,50}$ Based on the observation that body fat appears to be associated with a higher risk of distal colon tumors, a plausible hypothesis is that obesity and its associated process may influence risk via the chromosomal instability pathway. However, to our knowledge, this has not been investigated in any population-based studies. It is clear that the association between metabolic and hormonal risk factors and CRC is complex, and more research in this area is needed to elucidate clear mechanisms and how these mechanisms might differ with respect to tumor subsite.

In this Dutch population, body fat-especially abdominal fat, as indicated by trouser size was associated with tumors of the distal colon in men. In women, the influence of body fat on CRC risk may differ according to level of physical activity. Height was associated with $\mathrm{CRC}$ risk only in women. More research is needed to fully elucidate how body size may influence subsite-specific pathways of CRC carcinogenesis. 


\section{Reference}

1. World Cancer Research Fund / American Institute for Cancer Research. Food, Nutrition, Physical Activity, and the Prevention of Cancer: a Global Perspective. Washington DC: AICR, 2007.

2. Morois, S., Mesrine, S., Josset, M., ClavelChapelon, F. \& Boutron-Ruault, M. C. Anthropometric factors in adulthood and risk of colorectal adenomas: The French E3N-EPIC prospective cohort. Am J Epidemiol 172, 1166-1180 (2010).

3. Li, F. Y. \& Lai, M. D. Colorectal cancer, one entity or three. J Zhejiang Univ Sci B 10, 219-29 (2009).

4. Breivik, J. et al. Different genetic pathways to proximal and distal colorectal cancer influenced by sex-related factors. Int J Cancer 74, 664-669 (1997).

5. Lindblom, A. Different mechanisms in the tumorigenesis of proximal and distal colon cancers. Curr Opin Oncol 13, 63-69 (2001).

6. Caan, B. J. et al. Body size and the risk of colon cancer in a large case-control study. Int J Obes Relat Metab Disord 22, 178-184 (1998).

7. Gerhardsson de Verdier, M., Hagman, U., Steineck, G., Rieger, A. \& Norell, S. E. Diet, body mass and colorectal cancer: a case-referent study in Stockholm. Int J Cancer 46, 832-838 (1990).

8. Russo, A. et al. Body size and colorectal-cancer risk. Int J Cancer 78, 161-165 (1998).

9. Slattery, M. L. et al. Energy balance and colon cancer--beyond physical activity. Cancer Res 57, 75-80 (1997).

10. West, D. W. et al. Dietary intake and colon cancer: sex- and anatomic site-specific associations. Am J Epidemiol 130, 883-894 (1989).

11. Laake, I. et al. A prospective study of body mass index, weight change, and risk of cancer in the proximal and distal colon. Cancer Epidemiol Biomarkers Prev 19, 1511-1522 (2010).

12. Terry, P., Giovannucci, E., Bergkvist, L., Holmberg, L. \& Wolk, A. Body weight and colorectal cancer risk in a cohort of Swedish women: relation varies by age and cancer site. $\mathrm{Br} J$ Cancer 85, 346-349 (2001).

13. Oxentenko, A. S. et al. Body size and incident colorectal cancer: a prospective study of older women. Cancer Prev Res 3, 1608-1620 (2010).

14. Pischon, T. et al. Body size and risk of colon and rectal cancer in the European Prospective Investigation Into Cancer and Nutrition (EPIC). J Natl Cancer Inst 98, 920-931 (2006).

15. Pischon, T., Nöthlings, U. \& Boeing, H. Obesity and cancer. Proc Nutr Soc 67, 128-145 (2008).

16. Derks, S. et al. Integrated analysis of chromosomal, microsatellite and epigenetic instability in colorectal cancer identifies specific associations between promoter methylation of pivotal tumour suppressor and DNA repair genes and specific chromosomal alterations. Carcinogenesis 29, 434439 (2008).

17. Barault, L. et al. Hypermethylator phenotype in sporadic colon cancer: study on a populationbased series of 582 cases. Cancer Res 68, 8541-8546 (2008).

18. Samowitz, W. S. et al. Evaluation of a large, population-based sample supports a $\mathrm{CpG}$ island methylator phenotype in colon cancer. Gastroenterology 129, 837-845 (2005).

19. Weisenberger, D. J. et al. CpG island methylator phenotype underlies sporadic microsatellite instability and is tightly associated with BRAF mutation in colorectal cancer. Nat Genet 38, 787-793 (2006).

20. Campbell, P. T. et al. Case-control study of overweight, obesity, and colorectal cancer risk, overall and by tumor microsatellite instability status. $J$ Natl Cancer Inst 102, 391-400 (2010).

21. Hughes, L. A. et al. Body size, physical activity and risk of colorectal cancer with or without the CpG island methylator phenotype (CIMP). PloS One 6, e18571 (2011).

22. Slattery, M. L. et al. Diet and lifestyle factor associations with $\mathrm{CpG}$ island methylator phenotype and BRAF mutations in colon cancer. Int J Cancer 120, 656-663 (2007).

23. Van den Brandt, P. A. et al. A large-scale prospective cohort study on diet and cancer in The Netherlands. J Clin Epidemiol 43, 285-95 (1990).

24. Goldbohm, R. A. et al. Validation of a dietary questionnaire used in a large-scale prospective cohort study on diet and cancer. Eur J Clin Nutr 48, 253-65 (1994).

25. Goldbohm, R. A. et al. Reproducibility of a food frequency questionnaire and stability of dietary habits determined from five annually repeated measurements. Eur J Clin Nutr 49, 420-9 (1995).

26. Van den Brandt, P. A., Schouten, L. J., Goldbohm, R. A., Dorant, E. \& Hunen, P. M. Development of a record linkage protocol for use in the Dutch Cancer Registry for Epidemiological Research. Int J Epidemiol 19, 553-558 (1990).

27. Goldbohm, R. A., van den Brandt, P. A. \& Dorant, E. Estimation of the coverage of Dutch municipalities by cancer registries and PALGA based on hospital discharge data. Tijdschr Soc Gezondheidsz 72, 80-84. (1994).

28. Hughes, L. A., Schouten, L. J., Goldbohm, R. A., van den Brandt, P. A. \& Weijenberg, M. P. Self- 
reported clothing size as a proxy measure for body size. Epidemiology 20, 673-6 (2009).

29. Simons, C. C. et al. Physical activity, occupational sitting time, and colorectal cancer risk in the Netherlands cohort study. Am J Epidemiol 177, 514-530 (2013).

30. Hettinger, T. H., Mueller, B. H. \& Gebhard, H. Ermittlung des Arbeitsenergieumsatzes bei Dynamisch Muskulaerer Arbeit. Dortmund, Germany: Bundesarbeit fuer Arbeitsschutz, 1989.

31. Pylypchuk, R. D., Schouten, L. J., Goldbohm, R. A., Schouten, H. C. \& van den Brandt, P. A. Body mass index, height, and risk of lymphatic malignancies: a prospective cohort study. Am J Epidemiol 170, 297-307 (2009).

32. Slattery, M. L. \& Potter, J. D. Physical activity and colon cancer: confounding or interaction? Med Sci Sports Exerc 34, 913-919 (2002).

33. De Vogel, S. et al. Associations of dietary methyl donor intake with MLH1 promoter hypermethylation and related molecular phenotypes in sporadic colorectal cancer. Carcinogenesis 29, 17651773 (2008).

34. Bolton-Smith, C., Woodward, M., Tunstall-Pedoe, H. \& Morrison, C. Accuracy of the estimated prevalence of obesity from self-reported height and weight in an adult Scottish population. J Epidemiol Community Health 54, 143-148 (2000).

35. Nyholm, M. et al. The validity of obesity based on self-reported weight and height: Implications for population studies. Obesity 15, 197-208 (2007).

36. Spencer, E. A., Appleby, P. N., Davey, G. K. \& Key, T. J. Validity of self-reported height and weight in 4808 EPIC-Oxford participants. Public Heal Nutr 5, 561-5 (2002).

37. Wada, K. et al. Validity of self-reported height and weight in a Japanese workplace population. Int J Obes 29, 1093-9 (2005).

38. McKeown-Eyssen, G. Epidemiology of colorectal cancer revisited: are serum triglycerides and/or plasma glucose associated with risk? Cancer Epidemiol Biomarkers Prev 3, 687-695 (1994).

39. Silvera, S. A., Jain, M., Howe, G. R., Miller, A. B. \& Rohan, T. E. Energy balance and breast cancer risk: a prospective cohort study. Breast Cancer Res Treat 97, 97-106 (2006).

40. Slattery, M. L., Caan, B. J., Benson, J. \& Murtaugh, M. Energy balance and rectal cancer: an evaluation of energy intake, energy expenditure, and body mass index. Nutr Cancer 46, 166-171 (2003).

41. Okasha, M., Gunnell, D., Holly, J. \& Davey Smith, G. Childhood growth and adult cancer. Best Pract Res. Clin Endocrinol Metab 16, 225-241 (2002).

42. Engeland, A., Tretli, S., Austad, G. \& Bjørge, T. Height and body mass index in relation to colorectal and gallbladder cancer in two million Norwegian men and women. Cancer Causes Control 16, 987-996 (2005).

43. Wei, E. K. et al. Comparison of risk factors for colon and rectal cancer. Int J Cancer 108, 433-442 (2004).

44. Hughes, L. A. et al. Childhood and adolescent energy restriction and subsequent colorectal cancer risk: results from the Netherlands Cohort Study. Int J Epidemiol 39, 1333-1344 (2010).

45. Monteiro, R. \& Azevedo, I. Chronic inflammation in obesity and the metabolic syndrome. Mediators Inflamm 2010, (2010).

46. Elwing, J. E., Gao, F., Davidson, N. O. \& Early, D. S. Type 2 diabetes mellitus: the impact on colorectal adenoma risk in women. Am J Gastroenterol 101, 1866-1871 (2006).

47. Giovannucci, E. \& Michaud, D. The role of obesity and related metabolic disturbances in cancers of the colon, prostate, and pancreas. Gastroenterology 132, 2208-2225 (2007).

48. Hu, F. B. et al. Prospective study of adult onset diabetes mellitus (type 2) and risk of colorectal cancer in women. J Natl Cancer Inst 91, 542-547 (1999).

49. Jass, J. R. Classification of colorectal cancer based on correlation of clinical, morphological and molecular features. Histopathology 50, 113-30 (2007).

50. Slattery, M. L. et al. Colon tumor mutations and epigenetic changes associated with genetic polymorphism: insight into disease pathways. Mutat Res 660, 12-21 (2009). 


\section{Physical activity, occupational sitting time, and colorectal cancer risk in the Netherlands Cohort Study}

Colinda CJM Simons, Laura AE Hughes, Manon van Engeland, R Alexandra Goldbohm, Piet A van den Brandt and Matty P Weijenberg

Am J Epidemiol. 2013 Mar 15;177(6):514-30. 


\section{-Abstract-}

Background: We investigated occupational energy expenditure and sitting time in the longest held job (in men only), non-occupational physical activity, and former sports participation in relation to colorectal cancer endpoints.

Methods: The Netherlands Cohort Study includes 120,852 participants who completed a selfadministered questionnaire in 1986 when they were 55-69 years old. By 2002, 1,819 male and 1,366 female colorectal cancer cases were available for case-cohort analyses.

Results: In men, higher occupational energy expenditure levels and fewer occupational sitting hours were associated with decreased hazard ratios for colon cancer, particularly distal colon cancer (occupational energy expenditure of $\geq 12$ versus $<8 \mathrm{~kJ} /$ minute, hazard ratio $(\mathrm{HR})=0.71$, $95 \%$ confidence interval (CI): $0.52,0.97 ; P$-trend $=0.01$; occupational sitting hours of $<2$ versus $6-8$ hours $/$ day, $\mathrm{HR}=0.63,95 \% \mathrm{CI}: 0.48,0.83 ; P$-trend $=0.001$ ). The median duration of the longest held job for male subcohort members was 29 years. Non-occupational physical activity was inconsistently associated with colorectal cancer endpoints in men, and it was inversely associated with colon cancer in women, particularly distal colon cancer ( $>90$ versus $\leq 30 \mathrm{~min} /$ day, $\mathrm{HR}=0.69,95 \% \mathrm{CI}: 0.50,0.96 ; P$-trend $=0.06)$, and rectal cancer $(>90$ versus $\leq 30 \mathrm{~min} / \mathrm{day}, \mathrm{HR}=$ $0.59,95 \%$ CI: $0.39,0.90 ; P$-trend $=0.02)$.

Conclusion: Regular long-term physical activity and fewer sitting hours may protect against colon cancer, particularly distal colon cancer; results for rectal cancer were mixed. 


\subsection{Introduction}

Health recommendations include participating in moderate physical activity for at least 30 minutes every day and limiting sedentary habits. ${ }^{1}$ With respect to colorectal cancer (CRC), physical activity has been associated with a decreased colon cancer risk with indications of linear dose-response patterns; results for rectal cancer are inconsistent. ${ }^{2-10}$ It is, therefore, important to investigate different types and aspects of physical activity and sedentary behavior in relation to the subsitespecific CRC risk.

Several mechanisms may underlie the inverse association between physical activity and CRC risk. Physical activity may prevent overweight, improve immune function, reduce free radicals, increase prostaglandins, increase gut motility, improve insulin sensitivity, reduce insulin and insulin-like growth factor 1 levels, and increase the level of insulin-like growth factor binding protein 3.2,11 Any effect of physical activity on insulin-like growth factors may be especially important during puberty, when most bodily growth occurs. Intriguing in this respect is the finding of an increased CRC risk with increasing height, independent of other factors. ${ }^{12-16}$ It is, therefore, also interesting to elucidate the influence of physical activity during specific age periods.

To help clarify the unresolved issues, we analyzed occupational energy expenditure, occupational sitting time, baseline nonoccupational physical activity, and former sports participation in relation to CRC risk within the Netherlands Cohort Study. The measures we investigated cover a large part of participants' lifetime physical activity, and the Netherlands Cohort Study made possible sexspecific analyses per colorectal cancer subsite.

\subsection{Methods}

\subsubsection{Study population and design}

The Netherlands Cohort Study includes 120,852 participants who completed a selfadministered questionnaire at baseline in 1986, when they were $55-69$ years old. ${ }^{17}$ We used a case-cohort approach for efficiency reasons: 5,000 subcohort members who were randomly selected immediately after baseline were followed up for vital status to estimate the person-time at risk, whereas incident cancer cases were enumerated for the entire cohort. Participants who reported a history of cancer (other than skin cancer) at baseline were excluded. Subcohort members contributed to the person-time at risk until the end of follow-up or until censoring. Censoring occurred when a subcohort member became a cancer case, died, or was lost to follow-up. Follow-up of cancer cases was performed through linkage with the population-based cancer registry and the Netherlands national pathology database (PALGA). ${ }^{18,19}$ The estimated completeness of follow-up was more than $96 \%{ }^{20}$ for incident cancer cases and $\sim 100 \%$ for vital status. The baseline questionnaire included a 150 -item semi-quantitative food frequency questionnaire. Nutrient intake was calculated by using the Dutch food composition table of 1986-1987. ${ }^{21}$ The food frequency questionnaire was found to rank individuals adequately according to dietary intake ${ }^{22}$ and was shown to be a good indicator of dietary intake for at least 5 years. ${ }^{23}$

By 2002, 1,819 male and 1,366 female CRC cases (International Classification of Diseases for Oncology code 153) and 2,169 male and 2,247 female subcohort members were available for analyses (excluding those with incomplete/inconsistent questionnaires). Among men, there were 511 proximal colon cancer cases, 608 distal colon cancer cases, and 464 rectal cancer cases. Among women, these 
numbers were 560,417 , and 252, respectively. The total number of male and female colon cancer cases was 1,165 and 1,009, respectively, and included cases that could not be classified as either proximal or distal colon cancer. Rectosigmoid cancer cases were included only when analyzing CRC risk overall.

The Netherlands Cohort Study has been approved by the review boards of the TNO (Dutch abbreviation for Toegepast Natuurwetenschappelijk Onderzoek) Nutrition and Food Research Institute and Maastricht University.

\subsubsection{Occupational physical activity}

Data on occupational energy expenditure and occupational sitting time were derived from self-reported occupational history. A maximum of five jobs with accompanying start and stop years could be reported. The rating system we used ${ }^{24}$ distinguishes among work of low activity (e.g. desk jobs, driving); moderate activity (e.g. jobs that involve standing or walking $\leq 3 \mathrm{~km} /$ hour, jobs that involve heavy use of $1 \mathrm{arm}$, and jobs that involve moderately heavy use of both arms); and high activity (e.g. jobs that involve standing or walking $>3$ $\mathrm{km} /$ hour, jobs that involve heavy use of both arms, and physical labor such as digging, bending, or heavy lifting). These job types correspond to occupational energy expenditure of $<8,8-12$, and $>12 \mathrm{~kJ} / \mathrm{min}$. Sitting time was categorized into sitting for $>6-8,2-6$, and $<2$ working hours/day.

We did not analyze these measures in women because we were unable to classify $64.8 \%$ of female subcohort members who never held paid employment, whose jobs were uncodable, or whose data were missing. We therefore do not consider these measures to be representative of occupational physical activity levels in women. In men, we focused on occupational physical activity in the longest held job because it reflects regular long-term physical activity; the median duration of the longest held job among male subcohort members was 29 years, and 50\% held their longest job until 1983 or later. (For comparison, the median duration and stop year of the longest held job among female subcohort members were 9 years and 1956, respectively.)

\subsubsection{Baseline non-occupational physical activity}

Classification of baseline non-occupational physical activity was based on two questions. The first (open-ended) question was, "How many minutes do you spend on average per day walking/cycling to your work, to go shopping, or to walk your dog?" The reported time spent per day was categorized as $\leq 10$, $>10-30,>30-60$, and $>60 \mathrm{~min} /$ day. The second question was, "How many hours of your leisure time do you spend on average per week on 1) recreational walking/cycling, 2) gardening/doing odd jobs, and 3) sports/gymnastics?" Possible answers were never, <1, 12 , and $>2$ hours/week. Answers on both questions were summed to obtain an overall measure with categories of $\leq 30,>30-60,>60$ 90 , and $>90 \mathrm{~min} /$ day.

\subsubsection{Former sports participation}

Former sports participation was assessed by the question, "In case you formerly played sports, please indicate what sport(s), was it in a competition system, how many hours per week did you spend on each sport (exercise included), and in what period?" No more than 3 sports could be reported. Participants were categorized as having played sports if information was available on the type(s) of sport(s) played, the number of hours per week spent on sports, or the time period of sports participation. The total duration of former sports participation was calculated by aggregating the calendar years for which an individual 
was active in (one or several) sports. Sports intensity, expressed as a metabolic equivalent of task score, was based on the sport type..$^{25}$ The number of hours per week spent on a sport was derived from questionnaire responses. We analyzed the intensity and number of hours per week of sports participation with respect to the longest played sport. Information on age and the period of sports participation was used to distinguish the timing of sports initiation as either before or after the onset of puberty. Puberty was defined as the period between ages 12 and 15 years in boys, and therefore the onset of puberty was considered to have occurred at 12 years of age in all boys. In girls, individual data on age at menarche were available (mean age at menarche: 13.7 years; range: 9-22 years), and puberty was defined as the period between two years before menarche and one year after menarche. Therefore, the timing of the onset of puberty was determined individually for each female participant.

\subsubsection{Physical activity patterns}

Men with low occupational energy expenditure or high occupational sitting time may be active outside their jobs and vice versa. Therefore, for men, we combined occupational measures with the baseline non-occupational physical activity measure. Low occupational energy expenditure and occupational sitting time were defined as $<8 \mathrm{~kJ} / \mathrm{min}$ and $>6-8$ hours/day, respectively, as these categories comprised $58.6 \%$ of the male subcohort. In accordance with health recommendations, ${ }^{1}$ low non-occupational physical activity was considered to be $\leq 30 \mathrm{~min} /$ day. Moreover, because sports activity is likely to be only a small part of total physical activity, we combined the occupational energy expenditure measure in men and the baseline nonoccupational physical activity measure in women with the measures of former sports participation and sports participation around puberty.

\subsubsection{Statistical analysis}

Cox regression analysis was used to estimate hazard ratios and $95 \%$ confidence intervals by using STATA software, version 9.0 (StataCorp LP, College Station, Texas). Sex-specific hazard ratios and $95 \%$ confidence intervals were estimated for comparability with previous studies. To account for the additional variance introduced by sampling the subcohort from the entire cohort, we estimated standard errors by using the robust Huber-White sandwich estimator. ${ }^{26}$ The proportional hazards assumption was tested by using the scaled Schoenfeld residuals. ${ }^{27}$ As this test easily yields significant results when dealing with large numbers and long follow-up (as in our cohort), the use of more than one method is recommended. ${ }^{28}$ We also visually inspected the -log-log-transformed hazard curves and performed analyses for $\leq 8$ and $>8$ years of follow-up.

In multivariable-adjusted models, we included potential confounders selected from the literature and those that introduced more than a $10 \%$ change in hazard ratios. On the basis of previous studies, we included the following potential confounders: age, family history of CRC, smoking status, body mass index (BMI, calculated as weight $(\mathrm{kg}) /$ height $\left.(\mathrm{m})^{2}\right)$, total energy intake, and intakes of alcohol, meat, and processed meat. Other covariates considered were socioeconomic status, self-reported diabetes, and intakes of fiber, folate, $\beta$ carotene, vitamin $B_{6}$, vitamin $C$, vitamin $E$, fruits, vegetables, water through foods and beverages, calcium, total fat (energyadjusted), magnesium, and heme iron. None of these covariates introduced a change of more than $10 \%$. 
To check whether associations were independent of other physical activity types, we additionally adjusted models testing occupational energy expenditure and occupational sitting time for baseline non-occupational physical activity and vice versa. We also adjusted models testing occupational energy expenditure for occupational sitting time and vice versa. Models for former sports participation were adjusted for occupational energy expenditure in men and baseline nonoccupational physical activity in women. Furthermore, we checked a possible influence of having held jobs of different physical activity levels by restricting analyses for occupational energy expenditure and occupational sitting time in the longest held job to men who held jobs of the same physical activity level. As BMI may be an intermediate factor in the studied associations, we compared results derived from models adjusted for BMI with those from models not adjusted for BMI. We repeated this analysis by using trouser/skirt size as a proxy measure for waist circumference $^{29}$ because physical activity has been associated with both BMI and waist circumference. ${ }^{30}$ To check for the influence of preclinical disease, we repeated our main analyses excluding the first two years of follow-up. Statistical significance was indicated by a twotailed $P$-value of $<0.05$.

\subsection{Results}

In both men and women, CRC cases were not evidently more or less physically active than subcohort members when comparing the percentage of individuals across physical activity categories (Table 1). The percentage of cases with a family history of CRC was clearly higher than the percentage of subcohort members with a family history of CRC. Age, smoking status, alcohol intake, BMI, and intakes of the considered dietary factors were comparable between cases and subcohort members.
As occupational energy expenditure and occupational sitting time may correlate, we compared distributions in Table 2. Distributions differed significantly. Men with a low occupational energy expenditure level were spread across categories of occupational sitting time, whereas almost all men with $>6-8$ occupational sitting hours/day had a low occupational energy expenditure level.

Multivariable-adjusted results are presented in Tables 3 and 4 for men and women, respectively. We present these results instead of the mutually adjusted results for ease of comparison with the literature. Although the proportional hazards assumption was violated on some occasions when tested, -log-logtransformed hazard curves appeared to be proportional upon visual inspection. Ageadjusted results were comparable (data not shown).

Higher occupational energy expenditure levels and less occupational sitting time were significantly associated with lower hazard ratios for colon cancer in men. Hazard ratios were significantly decreased and lowest for distal colon cancer in men who had an energy expenditure of $>12 \mathrm{~kJ} / \mathrm{min}$ versus $<8 \mathrm{~kJ} / \mathrm{min}$ (hazard ratio $(\mathrm{HR})=0.71 ; 95 \%$ confidence interval (95\% CI): 0.52, 0.97; $P$-trend $=0.01$ ) and in men who had $<2$ occupational sitting hours/day versus $>6-8$ hours $/$ day $(\mathrm{HR}=0.63$, 95\% CI: 0.48, 0.83; P-trend $=0.001)$. In contrast, a significant positive trend was observed across categories of occupational energy expenditure, showing increased hazard ratios for rectal cancer, although hazard ratios were not statistically significant. There was no association between occupational sitting time and rectal cancer risk in men.

Baseline non-occupational physical activity was significantly inversely associated with colon cancer and rectal cancer risk in women $(P$-trend $=0.01$ and 0.02 , respectively), but not 
Table 1. Baseline characteristics of subcohort members and colorectal cancer (CRC) in the Netherlands Cohort Study (1986-2002)

\begin{tabular}{|c|c|c|c|c|}
\hline & \multicolumn{2}{|c|}{ Men } & \multicolumn{2}{|c|}{ Women } \\
\hline & $\begin{array}{l}\text { Subcohort } \\
\left(n^{*}=2,070\right)\end{array}$ & $\begin{array}{l}\text { CRC cases } \\
\left(n^{*}=1,739\right)\end{array}$ & $\begin{array}{l}\text { Subcohort } \\
\left(n^{*}=2,078\right)\end{array}$ & $\begin{array}{l}\text { CRC cases } \\
\left(n^{*}=1,260\right)\end{array}$ \\
\hline & $\%$ & $\%$ & $\%$ & $\%$ \\
\hline \multicolumn{5}{|l|}{ Occupational physical activity } \\
\hline Energy expenditure longest held job & $(\mathrm{n} \dagger=1,835)$ & $(\mathrm{n} \dagger=1,600)$ & & \\
\hline$<8 \mathrm{~kJ} / \mathrm{min}$ & 59.0 & 61.6 & & \\
\hline $8-12$ & 25.9 & 24.8 & & \\
\hline$>12$ & 15.1 & 13.6 & & \\
\hline Sitting time longest held job & $(\mathrm{n} \dagger=1,835)$ & $(\mathrm{n} \dagger=1,600)$ & & \\
\hline$>6-8$ hours/day & 25.9 & 30.0 & & \\
\hline $2-6$ & 45.2 & 42.6 & & \\
\hline$<2$ & 28.9 & 27.4 & & \\
\hline \multicolumn{5}{|l|}{ Non-occupational physical activity } \\
\hline Total at baseline & $(\mathrm{n} \dagger=2,052)$ & $(\mathrm{n} \dagger=1,727)$ & $(\mathrm{n} \dagger=2,053)$ & $(\mathrm{n} \dagger=1,246)$ \\
\hline$\leq 30 \mathrm{~min} /$ day & 17.7 & 15.6 & 23.4 & 27.8 \\
\hline$>30-60$ & 31.1 & 31.7 & 31.6 & 30.0 \\
\hline$>60-90$ & 19.0 & 20.2 & 23.1 & 23.4 \\
\hline$>90$ & 32.2 & 32.5 & 22.0 & 18.8 \\
\hline \multicolumn{5}{|l|}{ Daily walking/cycling } \\
\hline$\leq 10 \mathrm{~min} /$ day & 33.5 & 33.5 & 29.1 & 31.5 \\
\hline$>10-30$ & 22.2 & 21.3 & 29.6 & 29.9 \\
\hline$>30-60$ & 23.3 & 24.8 & 28.6 & 28.0 \\
\hline$>60$ & 21.1 & 20.4 & 12.8 & 10.6 \\
\hline \multicolumn{5}{|l|}{ Recreational walking/cycling } \\
\hline Never & 7.6 & 6.4 & 8.5 & 9.3 \\
\hline$<1$ hours/week & 20.3 & 20.4 & 24.2 & 23.0 \\
\hline $1-2$ & 31.4 & 29.1 & 32.3 & 34.2 \\
\hline$>2$ & 40.7 & 44.1 & 35.0 & 33.5 \\
\hline \multicolumn{5}{|l|}{ Gardening/doing odd jobs } \\
\hline Never & 15.0 & 12.6 & 37.9 & 37.4 \\
\hline$<1$ hours/week & 13.9 & 12.2 & 18.8 & 18.5 \\
\hline $1-2$ & 21.6 & 23.3 & 20.7 & 22.8 \\
\hline$>2$ & 49.5 & 51.8 & 22.6 & 21.3 \\
\hline \multicolumn{5}{|l|}{ Sports/gymnastics } \\
\hline Never & 72.3 & 68.5 & 65.5 & 67.6 \\
\hline$<1$ hours/week & 11.0 & 13.1 & 13.5 & 10.8 \\
\hline $1-2$ & 8.6 & 9.5 & 14.5 & 15.7 \\
\hline$>2$ & 8.1 & 8.9 & 6.5 & 5.9 \\
\hline \multicolumn{5}{|l|}{ Sports history } \\
\hline \multicolumn{5}{|l|}{ Former sports participation } \\
\hline Never & 45.2 & 45.1 & 53.3 & 55.4 \\
\hline Ever & 54.8 & 54.9 & 46.7 & 44.6 \\
\hline
\end{tabular}




\begin{tabular}{|c|c|c|c|c|}
\hline & \multicolumn{2}{|c|}{ Men } & \multicolumn{2}{|c|}{ Women } \\
\hline & $\begin{array}{l}\text { Subcohort } \\
\left(\mathrm{n}^{*}=2,070\right)\end{array}$ & $\begin{array}{l}\text { CRC cases } \\
\left(\mathrm{n}^{*}=1,739\right)\end{array}$ & $\begin{array}{l}\text { Subcohort } \\
\left(\mathrm{n}^{*}=2,078\right)\end{array}$ & $\begin{array}{l}\text { CRC cases } \\
\left(n^{*}=1,260\right)\end{array}$ \\
\hline Total duration former sports & $(\mathrm{n} \dagger=2,011)$ & $(\mathrm{n} \dagger=1,697)$ & $(\mathrm{n} \dagger=1,988)$ & $(\mathrm{n} \uparrow=1,199)$ \\
\hline Never played sports & 46.5 & 46.3 & 55.7 & 58.2 \\
\hline$<11$ (years) & 23.7 & 22.9 & 23.9 & 21.3 \\
\hline$\geq 11$ & 29.7 & 30.8 & 20.4 & 20.5 \\
\hline Intensity longest played sport & $(\mathrm{n} \dagger=2,005)$ & $(\mathrm{n} \dagger=1,696)$ & $(\mathrm{n} \dagger=1,980)$ & $(\mathrm{n} \dagger=1,197)$ \\
\hline Never played sports & 46.7 & 46.3 & 55.9 & 58.3 \\
\hline$<7$ (METs $\ddagger)$ & 13.6 & 14.3 & 23.6 & 21.7 \\
\hline$\geq 7$ & 39.7 & 39.4 & 20.5 & 20.0 \\
\hline Hours/week longest played sport & $(\mathrm{n} \dagger=1,977)$ & $(\mathrm{n} \dagger=1,671)$ & $(\mathrm{n} \dagger=1,960)$ & $(\mathrm{n} \dagger=1,177)$ \\
\hline Never played sports & 47.3 & 47.0 & 56.5 & 59.3 \\
\hline$<3$ & 21.6 & 20.0 & 28.2 & 26.3 \\
\hline$\geq 3$ & 31.1 & 33.0 & 15.4 & 14.4 \\
\hline Sports around puberty & $(\mathrm{n} \dagger=2,011)$ & $(\mathrm{n} \dagger=1,697)$ & $(\mathrm{n} \dagger=1,980)$ & $(\mathrm{n} \dagger=1,196)$ \\
\hline Never played sports & 46.5 & 46.3 & 55.9 & 58.4 \\
\hline After puberty & 24.7 & 24.8 & 21.4 & 20.0 \\
\hline Started before puberty & 28.7 & 28.9 & 22.7 & 21.7 \\
\hline \multicolumn{5}{|l|}{ Potential confounders } \\
\hline Family history of CRC (yes) & 5.3 & 9.1 & 6.0 & 9.7 \\
\hline \multicolumn{5}{|l|}{ Smoking status } \\
\hline Never smoker & 12.8 & 11.7 & 57.6 & 58.4 \\
\hline Ex & 51.8 & 58.0 & 20.8 & 21.6 \\
\hline Current & 35.4 & 30.3 & 21.6 & 20.0 \\
\hline \multicolumn{5}{|l|}{ Alcohol intake } \\
\hline $0 \mathrm{~g} /$ day & 14.6 & 12.0 & 32.3 & 32.5 \\
\hline $0.1-29$ & 70.7 & 70.4 & 64.1 & 62.5 \\
\hline \multirow{2}{*}{$\geq 30$} & 14.7 & 17.6 & 3.6 & 5.1 \\
\hline & Mean (SD) & Mean (SD) & Mean (SD) & Mean (SD) \\
\hline Age (years) & $61.3(4.2)$ & $61.9(4.1)$ & $61.4(4.3)$ & $62.3(4.0)$ \\
\hline BMI $\left(\mathrm{kg} / \mathrm{m}^{2}\right)$ & $24.9(2.6)$ & $25.2(2.6)$ & $25.0(3.5)$ & $25.0(3.5)$ \\
\hline Meat intake (g/day) & $106(42.9)$ & $105(41.1)$ & $93.1(39.5)$ & $92.5(40.1)$ \\
\hline Processed meat intake (g/day) & $17.2(17.6)$ & $17.7(17.6)$ & $11.2(12.4)$ & $11.2(11.7)$ \\
\hline Total energy intake (kcal/day) & $2,172(510)$ & $2,148(489)$ & $1,688(397)$ & $1,679(304)$ \\
\hline
\end{tabular}

\footnotetext{
Abbreviations: BMI, body mass index (weight $(\mathrm{kg}) /$ height $\left.(\mathrm{m})^{2}\right)$; MET, metabolic equivalent of task; SD, standard deviation

* Excluding subcohort members and colorectal cancer cases with missing data on potential confounders.

$\dagger$ Numbers do not add up to the total number of subcohort members or cases because of missing values on exposure variables.

$\ddagger$ The MET score is the ratio between the metabolic rate during a specific physical activity and the resting metabolic rate (i.e. the metabolic rate while sitting quietly).
} 
Table 2. Cross tabulation of occupational energy expenditure and occupational sitting time in the longest held job of male subcohort members in the Netherlands Cohort Study (1986-2002)

\begin{tabular}{lrrr}
\hline & \multicolumn{3}{c}{ Occupational sitting time* $^{*}$} \\
\cline { 2 - 4 } $\begin{array}{l}\text { Occupational energy } \\
\text { expenditure* }\end{array}$ & $>6-8$ hours/day & $2-6$ & $<2$ \\
$\mathrm{n} \dagger(\%)$ & $\mathrm{n} \dagger(\%)$ & $23(4.3)$ \\
\hline$<8 \mathrm{~kJ} / \mathrm{min}$ & $472(99.4)$ & $588(70.8)$ & $299(56.4)$ \\
$8-12$ & $3(0.6)$ & $173(20.8)$ & $208(39.2)$ \\
$>12$ & 0 & $69(8.3)$ & 530 \\
Total & 475 & 830 & \\
\hline \hline
\end{tabular}

* $P$-value for the $\chi^{2}$ test was $<0.001$.

† Excluded were men with missing data on occupational energy expenditure, occupational sitting time, and potential confounders.

in men. Hazard ratios were significantly lower in women who reported $>90$ minutes versus $\leq 30$ minutes of daily baseline nonoccupational physical activity (HR for proximal colon cancer $=0.71,95 \% \mathrm{CI}: 0.53,0.96$; $\mathrm{HR}$ for distal colon cancer $=0.69,95 \%$ CI: 0.50 , 0.96; HR for rectal cancer $=0.59,95 \%$ CI: 0.39 , 0.90). When investigating non-occupational physical activity types, we found that hazard ratios for rectal cancer were lower in women who walked/cycled for $>60 \mathrm{~min} /$ day versus $\leq 10 \mathrm{~min} /$ day $(\mathrm{HR}=0.47,95 \% \mathrm{CI}: 0.27,0.83 ; P$ trend $=0.03$ ). No associations were observed among women for recreational walking/cycling, gardening/doing odd jobs, or participating in sports/gymnastics. In men, there were significant positive dose-response relationships between distal colon cancer risk and gardening/doing odd jobs $(>2$ hours/week versus never: $\mathrm{HR}=1.48,95 \% \mathrm{CI}$ : $1.08,2.03$; $P$-trend $=0.04$ ) and participating in sports/gymnastics ( $>2$ hours/week versus never: $\mathrm{HR}=1.52,95 \% \mathrm{CI}: 1.11,2.10 ; P$-trend $=$ $0.01)$, but there were no associations with other types of non-occupational physical activity. Former sports participation was not associated with CRC endpoints.

Further analyses (data not shown) demonstrated that associations with occupational energy expenditure and occupational sitting time were unchanged after adjustment for baseline non-occupational physical activity in men, and that associations were most pronounced for those with long follow-up. Models in which occupational energy expenditure was adjusted for occupational sitting time and vice versa showed attenuated hazard ratios. The hazard ratio for distal colon cancer remained significant in men who sat for 2-6 hours/day versus $>6-8$ hours/day. Analyses in men who had jobs of the same physical activity level produced similar results. Associations with baseline non-occupational physical activity were unchanged after adjustment for occupational energy expenditure in men and were evident in analyses for both short and long follow-up in both men and women. Former sports participation was not associated with CRC endpoints after adjustment for occupational energy expenditure in men and baseline non-occupational physical activity in women.

The results for physical activity patterns and CRC risk are presented in Tables 5 and 6 for men and women, respectively. Patterns indicating higher daily physical activity levels in men were significantly inversely associated with distal colon cancer risk. Associations were less clear than when considering physical activity measures separately. Measures combining former sports participation with occupational energy expenditure in men and baseline non-occupational physical activity in women were significantly inversely associated 


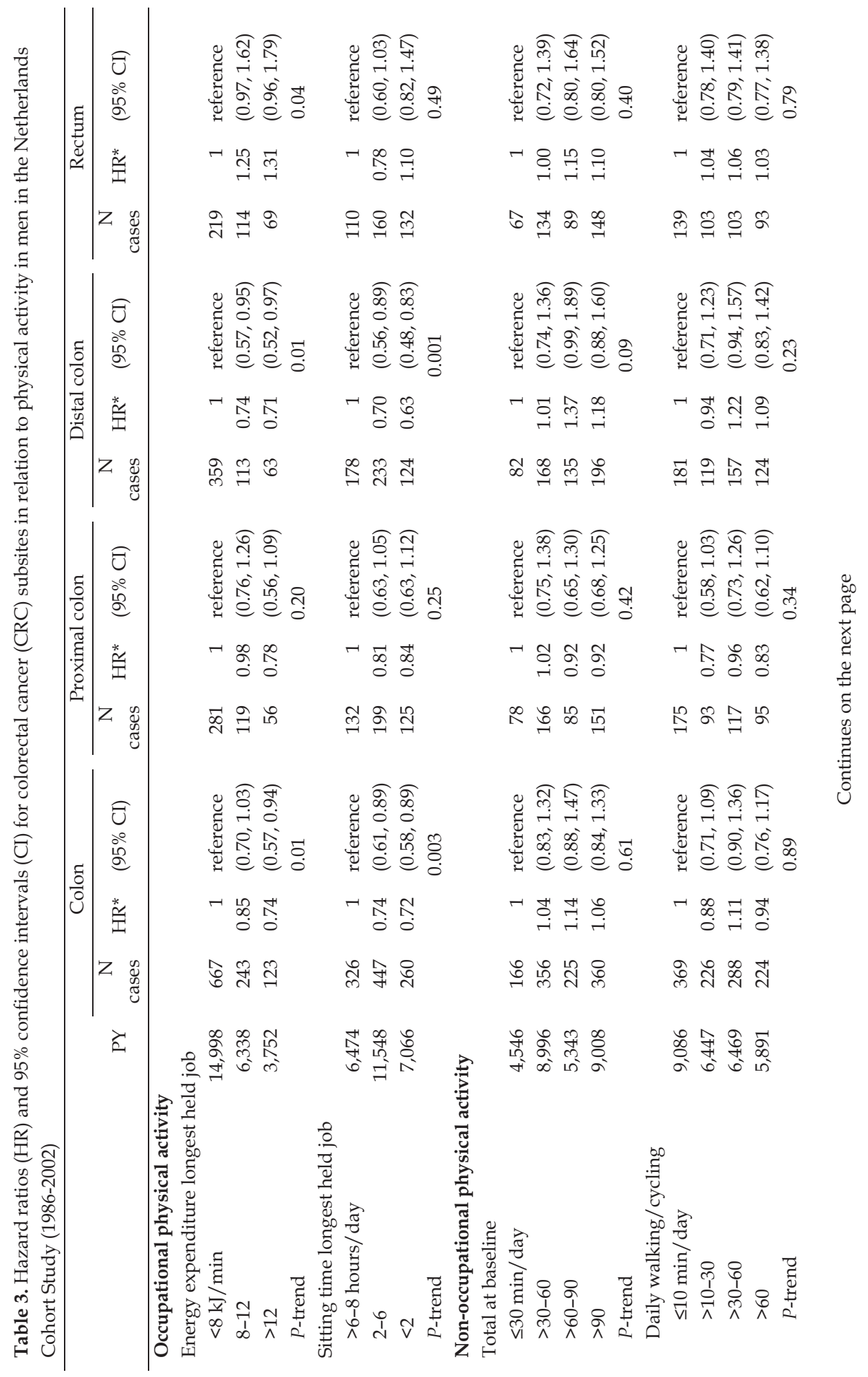




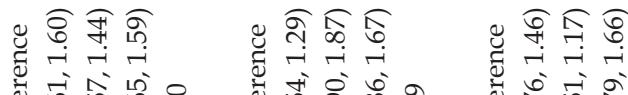

홀

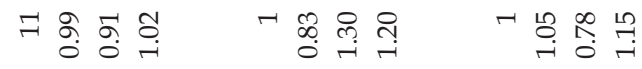

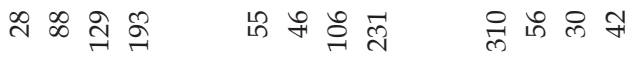

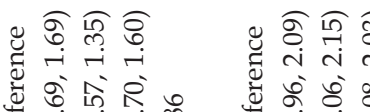

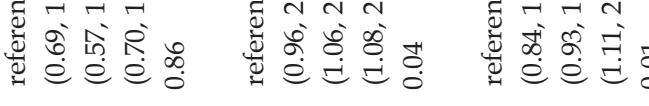

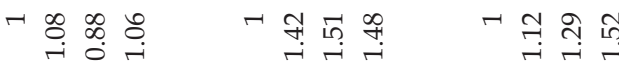

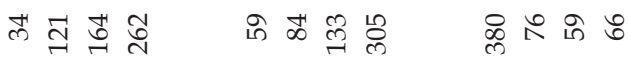

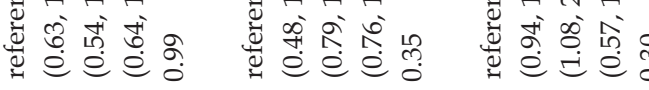

$\forall \underset{+\infty}{\infty} \underset{0}{\infty} \delta$

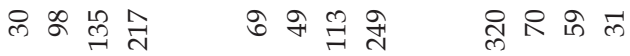

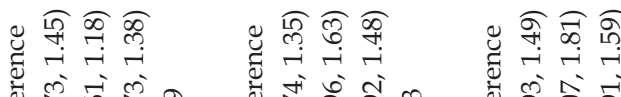

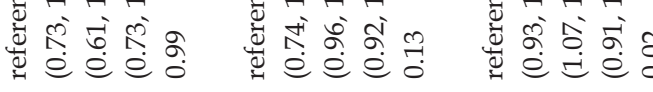

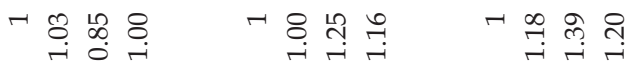

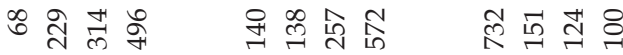

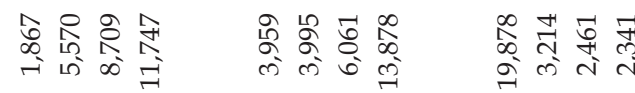

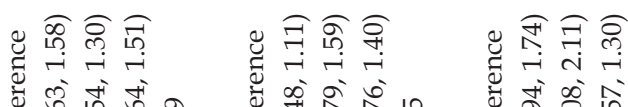

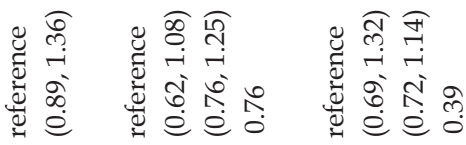

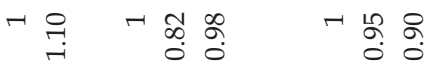

तั

๖

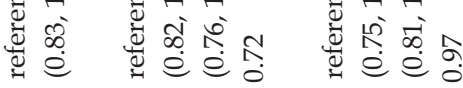

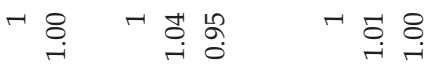

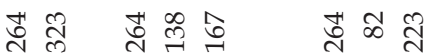

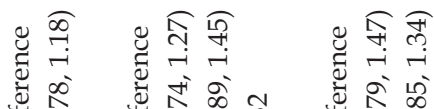

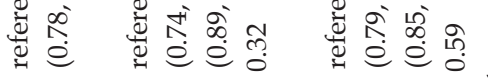

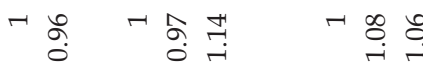

국

:

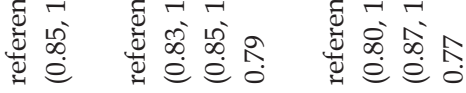

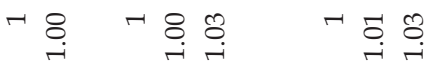

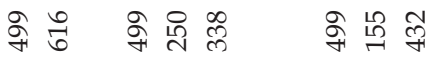

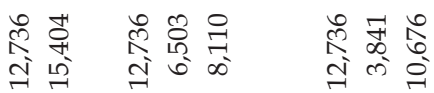

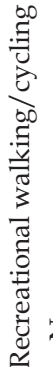

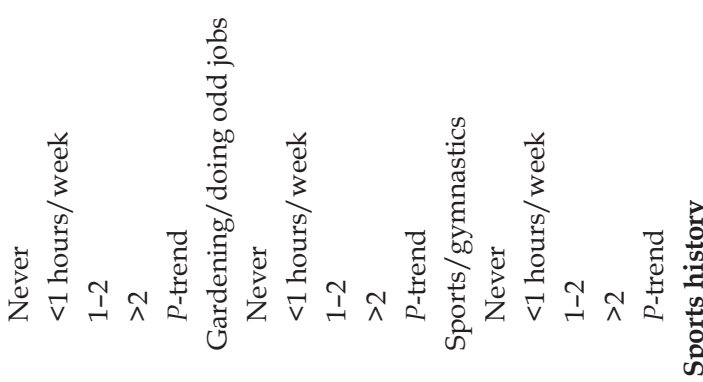




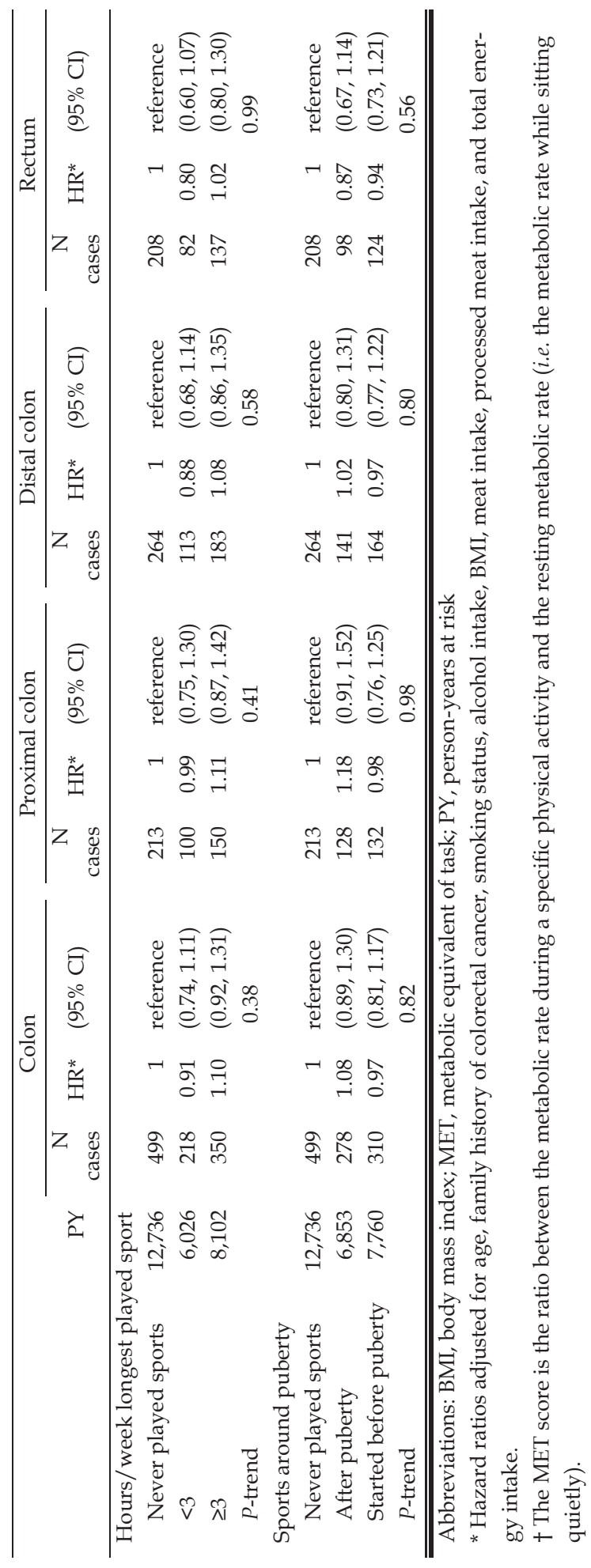




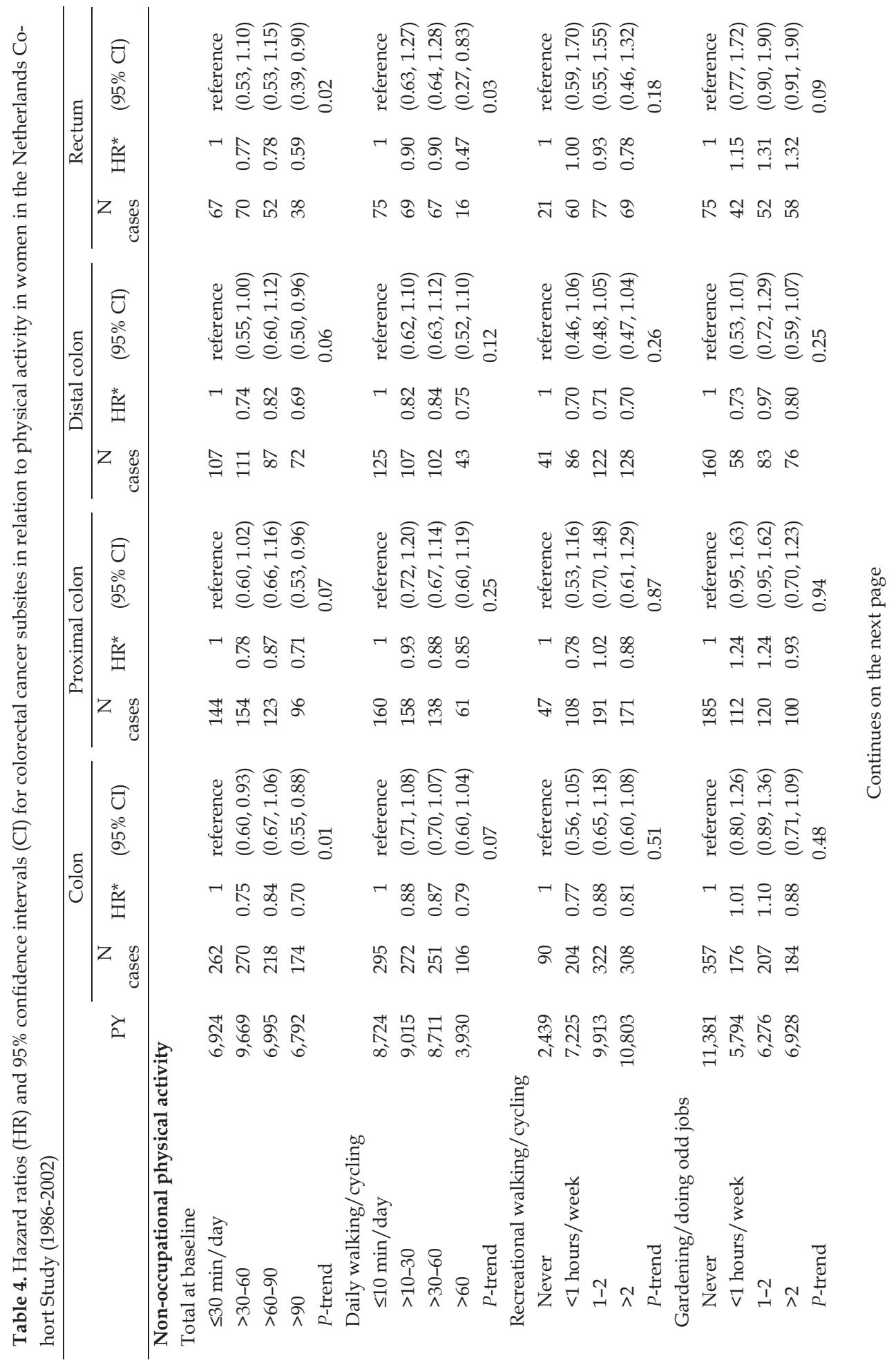




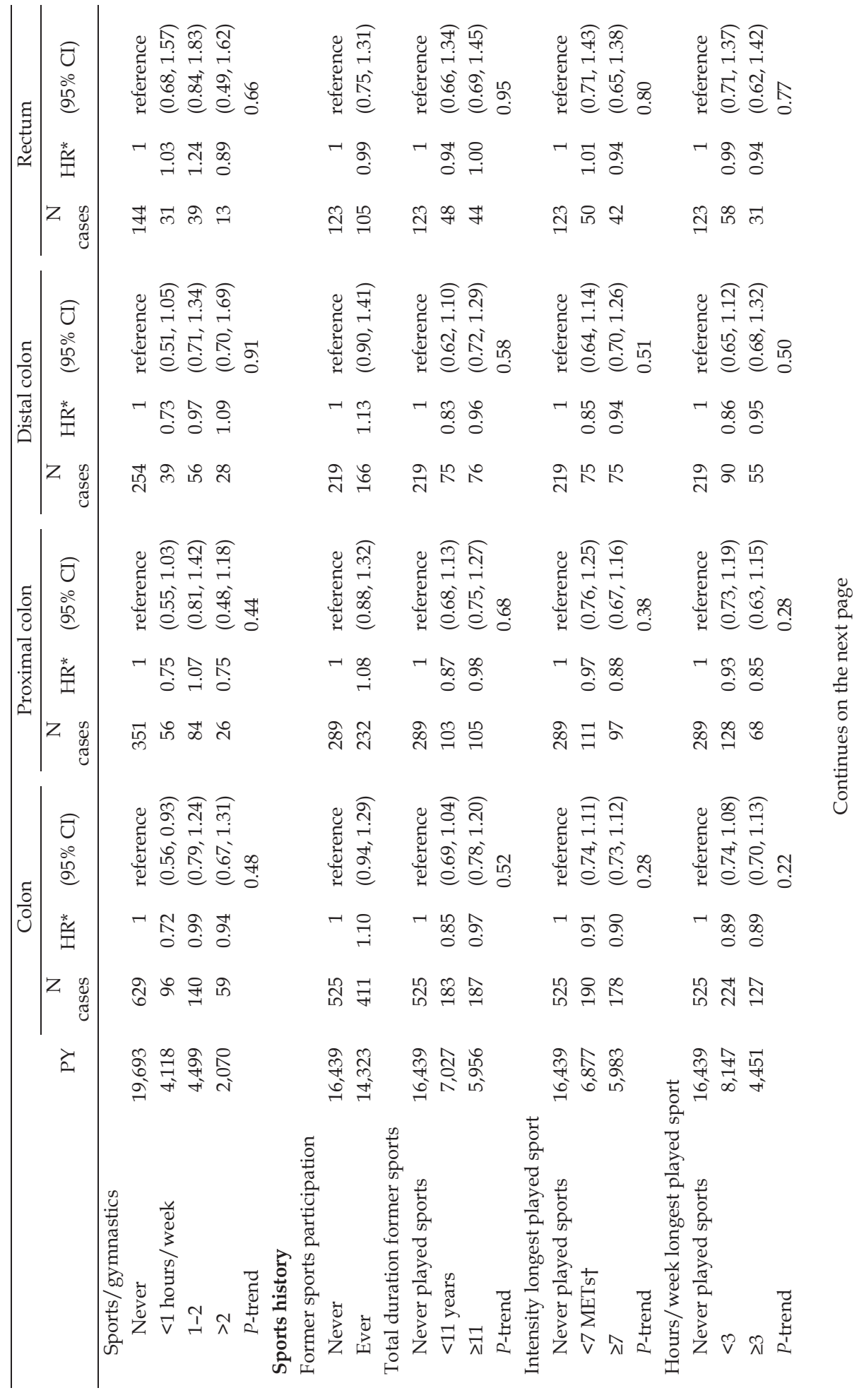




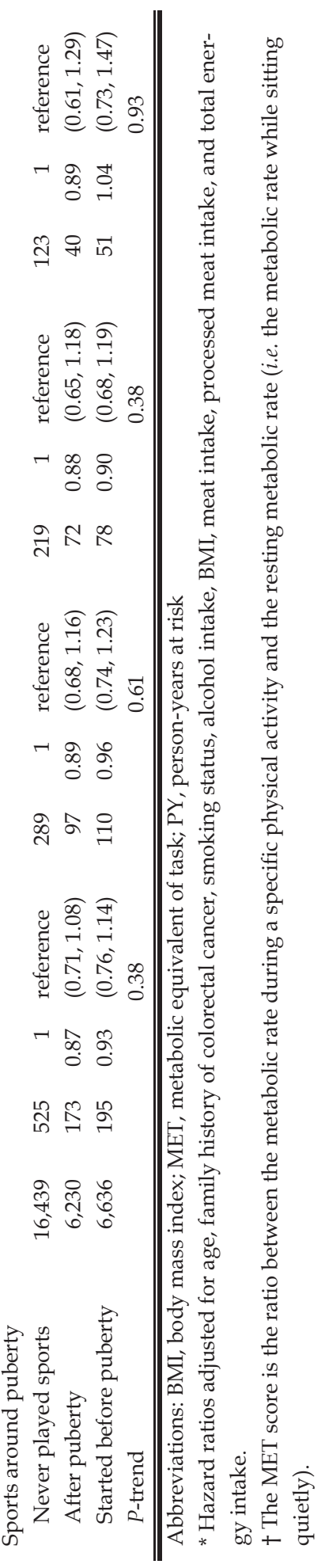




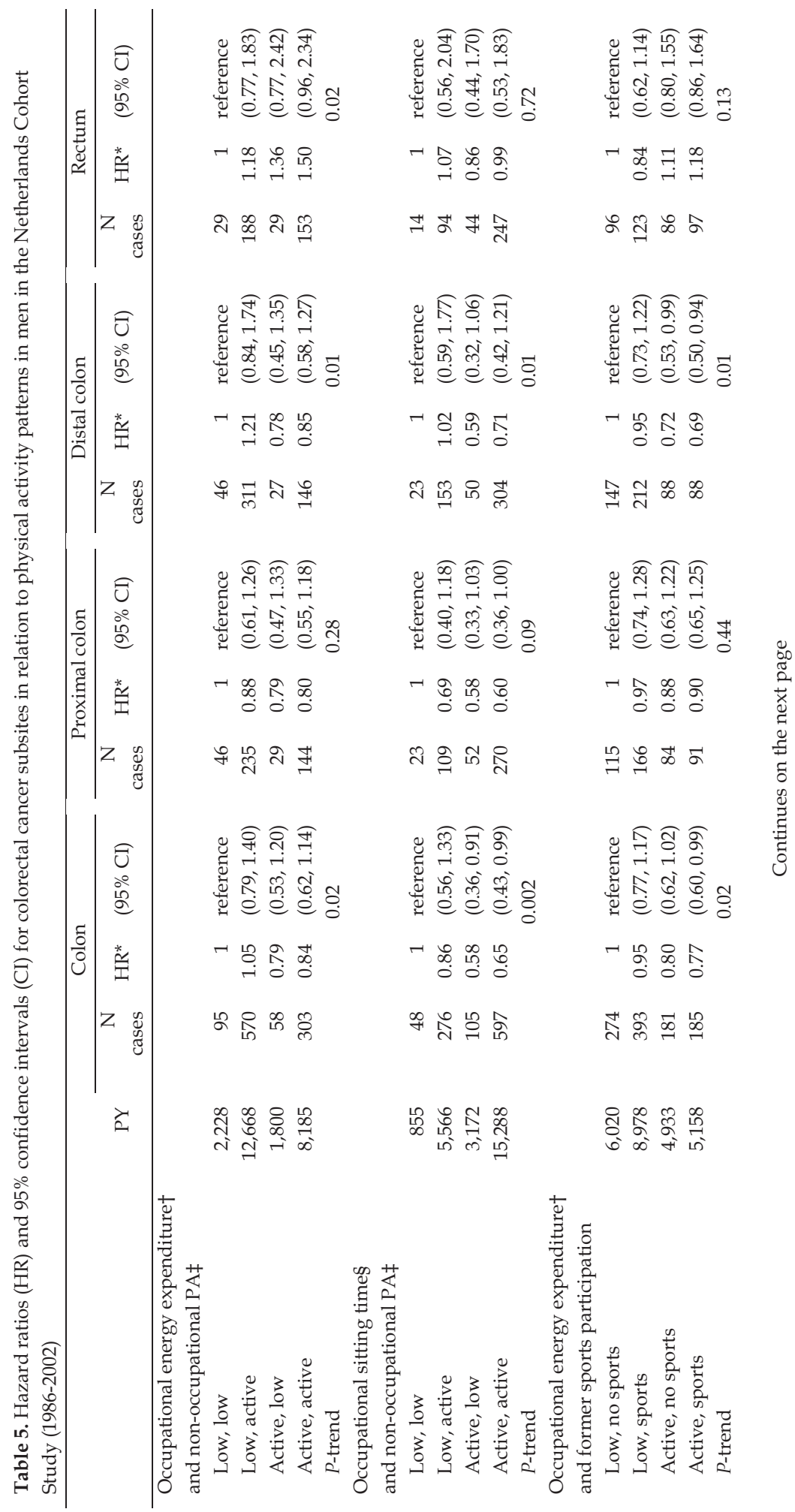




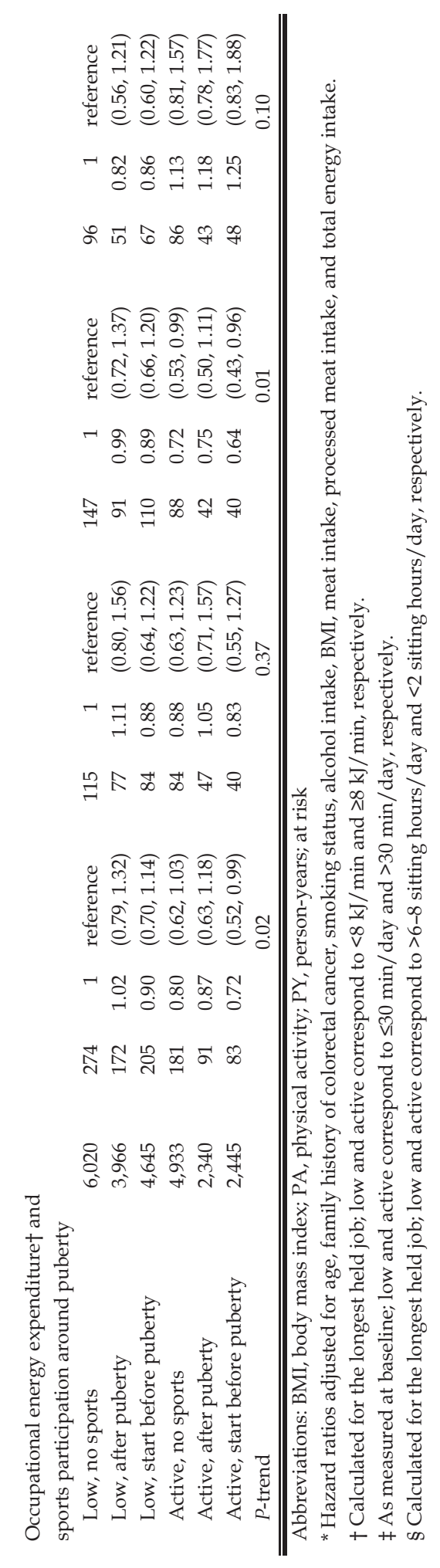




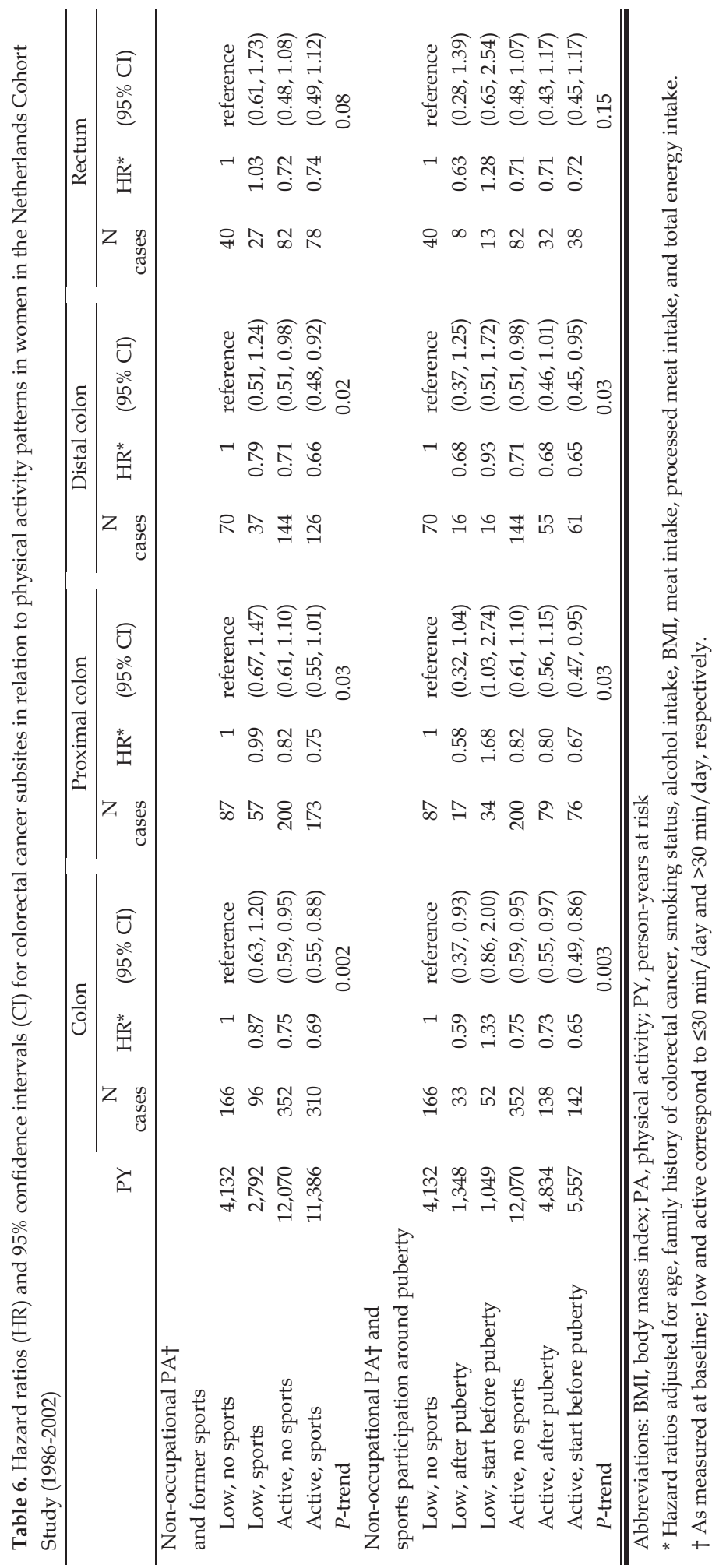


with colon cancer risk. When including the time period of sports participation, hazard ratios were lowest and significantly decreased in active participants who started playing sports before puberty compared with inactive participants who never played sports (in men: HR for distal colon cancer $=0.64,95 \%$ CI: 0.43 , 0.96; in women: HR for proximal colon cancer $=0.67,95 \%$ CI: $0.47,0.95$ and HR for distal colon cancer $=0.65,95 \%$ CI: $0.45,0.95)$. Conversely, there were a significantly increased hazard ratio for proximal colon cancer in inactive women who started playing sports before puberty $(\mathrm{HR}=1.68,95 \% \mathrm{CI}: 1.03,2.74)$ and a nonsignificantly increased hazard ratio for rectal cancer. In men, nonsignificantly increased hazard ratios for rectal cancer were also evident.

Finally, analyses did not indicate that either BMI or waist circumference was an intermediate factor. Exclusion of the first two years of follow-up produced comparable estimates.

\subsection{Discussion}

Our results are consistent with those of numerous studies suggesting that nonoccupational physical activity may protect against CRC, particularly colon cancer. ${ }^{2-10,31}$ Former sports participation was not associated with CRC risk. Previously, results have been inconsistent regarding rectal cancer risk, and it is not clear whether the inverse association with colon cancer risk is because of an inverse association with proximal or distal colon cancer risk or both. ${ }^{8}$ Differences in physical activity assessment likely contribute to this heterogeneity. ${ }^{8}$ Our study shows that physical activity may protect against distal colon cancer in particular, and that limiting daily sitting time may be important. In addition, regular long-term physical activity could be important when we consider that the occupational physical activity measure used in men was based on the longest held job, and half of the male subcohort held their longest job for 29 years or longer. In women, baseline nonoccupational physical activity is likely representative of a considerable time period, as $50.9 \%$ of female subcohort members described themselves as homemakers exclusively, and most others held jobs only briefly and in the distant past (the median duration and stop year of the longest held job were 9 years and 1956, respectively). It has been suggested previously that consistently high physical activity levels may confer the greatest decrease in colon cancer risk. 32,33

Special emphasis may be placed on our results regarding occupational sitting time because sedentary behavior is becoming more common and may differ in its effects from low levels of physical activity. We observed significantly different distributions across categories of occupational energy expenditure and occupational sitting time. Hazard ratios for occupational sitting time were less attenuated when adjusting for occupational energy expenditure than vice versa, suggesting a potential independent effect of sitting time. Lynch ${ }^{31}$ reported that sedentary behavior may influence risk through mechanisms that are both similar to and different from those of physical activity, as rat studies found that sedentary behavior had a greater effect than exercise training on lipoprotein lipase regulation, and expression of certain genes in skeletal muscle was shown to be most sensitive to inactivity.

Chromosomal instability, a tumor phenotype associated with chromosomal gains and losses and structural rearrangements, may possibly explain why physical activity was associated mainly with distal colon cancer risk. Chromosomal instability occurs more often in cancers in the distal colon than in other sublocalizations, ${ }^{34}$ and environmental factors such as physical activity may act predominantly through the chromosomal instability pathway. The findings of a recent exercise inter- 
vention trial in which exercising affected colon crypt cell proliferation ${ }^{35}$ and expression of the apoptotic-regulating proteins B-cell lymphoma-2 and B-cell lymphoma-2-associated X protein in colon crypts ${ }^{36}$ indicate chromosomal instability involvement. Exercising did not affect colon mucosal prostaglandin concentrations $^{37}$ and C-reactive protein levels, ${ }^{38}$ suggesting that inflammation is less important.

Although the influence of physical activity on CRC risk may differ by subsite of cancer, sex, and type, intensity level, and duration of physical activity, our study yielded a few surprising findings. First, regarding rectal cancer risk, a positive dose-response relationship was observed with occupational energy expenditure in men. This result may have been influenced by residual confounding, as there may have been occupational exposures that are risk factors for rectal cancer (e.g. asbestos $\left.{ }^{39}\right)$ for which we could not adjust.

Second, it is unclear why no associations with CRC risk were observed in men for daily walking/cycling and recreational walking/cycling, although positive associations with distal colon cancer risk emerged for gardening/doing odd jobs and participating in sports/gymnastics. These latter positive associations contradicted our hypothesis but might be explained if men with inactive jobs compensated for their lack of occupational physical activity in their spare time, thus reporting high non-occupational physical activity levels while having been inactive most of the time.

Men who were retired at baseline $(43.7 \%$ of the male subcohort) may also have reported high non-occupational physical activity levels that were not representative of lifetime levels. However, cross tabulation of gardening/doing odd jobs and participating in sports/ gymnastics on one hand and occupa- tional energy expenditure on the other hand in male subcohort members and in male subcohort members who were retired at baseline did not support this. In women, the inverse associations between non-occupational physical activity and colon and rectal cancer risk were consistent with our hypothesis. The fact that daily walking/cycling (but not other types of non-occupational physical activity) was inversely associated with rectal cancer risk supports the importance of regular physical activity. Results also differed between men and women in previous cohort studies, yet it remains difficult to draw conclusions regarding sex differences. ${ }^{8}$

Third, when analyzing physical activity patterns, we observed decreased hazard ratios for colon cancer in active women who started playing sports before puberty, yet increased hazard ratios in inactive women who started playing sports before puberty as compared with inactive women who never played sports. Based on this finding, it is difficult to determine whether puberty is a critical period for physical activity that could later influence CRC risk. Previously, we found that participants in the Netherlands Cohort Study who were exposed to famine in childhood and adolescence during the "Hunger Winter" of 1944-1945 were at a significantly decreased risk of CRC characterized by the promoter hypermethylation phenotype. ${ }^{40,41}$ It could be that sports participation was not an extreme enough early life exposure to influence future cancer risk regardless of physical activity levels in adulthood.

Strengths of our study include the prospective design and high completeness of follow-up, making selection and information bias unlikely. Our physical activity measures also cover a large part of participants' total physical activity, which enabled the exploration of physical activity patterns. Limitations include the single baseline measurement; that is, changes in 
physical activity levels and in levels of potential confounders over time may have influenced results. However, in sensitivity analyses, results proved fairly robust. Another limitation may be that physical activity was self-reported and not tested for validity and reproducibility. According to Ferrari et al.,42 measurement errors in self-reported physical activity can attenuate associations, and age and BMI may influence validity, with higher coefficients observed for younger individuals and individuals below sex-specific BMI medians. Although our cohort consists of elderly individuals, our self-reported physical activity measures represent long-term physical activi- ty, which is difficult to assess otherwise. Furthermore, an expert assessment scheme was used to derive occupational physical activity from self-reported occupational history, and individuals have been shown to be able to report accurately on their occupational history. ${ }^{43}$

In conclusion, our findings support an inverse association between physical activity and colon cancer risk, in particular distal colon cancer risk, whereas results for rectal cancer were mixed. Measures of regular long-term physical activity seemed to be the best predictors of risk, and it may be important to avoid sedentary behavior. 
1. World Cancer Research Fund / American Institute for Cancer Research. Food, Nutrition, Physical Activity, and the Prevention of Cancer: a Global Perspective. Washington DC: AICR, 2007.

2. Slattery, M. L. Physical activity and colorectal cancer. Sports Med 34, 239-252 (2004).

3. Samad, A. K., Taylor, R. S., Marshall, T. \& Chapman, M. A. A meta-analysis of the association of physical activity with reduced risk of colorectal cancer. Color Dis 7, 204-213 (2005).

4. Harriss, D. J. et al. Physical activity before and after diagnosis of colorectal cancer: disease risk, clinical outcomes, response pathways and biomarkers. Sports Med 37, 947-960 (2007).

5. Harriss, D. J. et al. Lifestyle factors and colorectal cancer risk (2): a systematic review and metaanalysis of associations with leisure-time physical activity. Color Dis 11, 689-701 (2009).

6. Pan, S. Y. \& DesMeules, M. Energy intake, physical activity, energy balance, and cancer: epidemiologic evidence. Methods Mol Biol 472, 191-215 (2009).

7. Wolin, K. Y., Yan, Y., Colditz, G. A. \& Lee, I. M. Physical activity and colon cancer prevention: a meta-analysis. Br J Cancer 100, 611-616 (2009).

8. Spence, R. R., Heesch, K. C. \& Brown, W. J. A systematic review of the association between physical activity and colorectal cancer risk. Scand J Med Sci Sports 19, 764-781 (2009).

9. Friedenreich, C. M., Neilson, H. K. \& Lynch, B. M. State of the epidemiological evidence on physical activity and cancer prevention. Eur J Cancer 46, 2593-2604 (2010).

10. Wolin, K. Y. \& Tuchman, H. Physical activity and gastrointestinal cancer prevention. Recent Results Cancer Res 186, 73-100 (2011).

11. McTiernan, A. Mechanisms linking physical activity with cancer. Nat Rev Cancer 8, 205-211 (2008).

12. Shimizu, N. et al. Height, weight, and alcohol consumption in relation to the risk of colorectal cancer in Japan: a prospective study. Br J Cancer 88, 1038-1043 (2003).

13. Pischon, T. et al. Body size and risk of colon and rectal cancer in the European Prospective Investigation Into Cancer and Nutrition (EPIC). J Natl Cancer Inst 98, 920-931 (2006).

14. Sung, J., Song, Y. M., Lawlor, D. A., Smith, G. D. \& Ebrahim, S. Height and site-specific cancer risk: A cohort study of a Korean adult population. Am J Epidemiol 170, 53-64 (2009).

15. Green, J. et al. Height and cancer incidence in the Million Women Study: prospective cohort, and meta-analysis of prospective studies of height and total cancer risk. Lancet Oncol 12, 785-794 (2011).

16. Hughes, L. A. E. et al. Body size and colorectal cancer risk after 16.3 years of follow-up: an analysis from the Netherlands Cohort Study. Am J Epidemiol 174, 1127-1139 (2011).

17. Van den Brandt, P. A. et al. A large-scale prospective cohort study on diet and cancer in The Netherlands. J Clin Epidemiol 43, 285-95 (1990).

18. Casparie, M. et al. Pathology databanking and biobanking in The Netherlands, a central role for PALGA, the nationwide histopathology and cytopathology data network and archive. Cell Oncol 29, 19-24 (2007).

19. Van den Brandt, P. A., Schouten, L. J., Goldbohm, R. A., Dorant, E. \& Hunen, P. M. Development of a record linkage protocol for use in the Dutch Cancer Registry for Epidemiological Research. Int J Epidemiol 19, 553-558 (1990).

20. Goldbohm, R. A., van den Brandt, P. A. \& Dorant, E. Estimation of the coverage of Dutch municipalities by cancer registries and PALGA based on hospital discharge data. Tijdschr Soc Gezondheidsz 72, 80-84. (1994).

21. Nevo Table. Dutch food composition table 19867. The Hague, Netherlands: Voorlichtingsbureau voor de Voeding, 1986.

22. Goldbohm, R. A. et al. Validation of a dietary questionnaire used in a large-scale prospective cohort study on diet and cancer. Eur J Clin Nutr 48, 253-65 (1994).

23. Goldbohm, R. A. et al. Reproducibility of a food frequency questionnaire and stability of dietary habits determined from five annually repeated measurements. Eur J Clin Nutr 49, 420-9 (1995).

24. Hettinger, T. H., Mueller, B. H. \& Gebhard, H. Ermittlung des Arbeitsenergieumsatzes bei Dynamisch Muskulaerer Arbeit. Dortmund, Germany: Bundesarbeit fuer Arbeitsschutz, 1989.

25. Ainsworth, B. E. et al. Compendium of physical activities: classification of energy costs of human physical activities. Med Sci Sports Exerc 25, 71-80 (1993).

26. Barlow, W. E. Robust variance estimation for the case-cohort design. Biometrics 50, 1064-1072 (1994).

27. Schoenfeld, D. Partial residuals for the proportional hazards regression model. Biometrika 69, 239-241 (1982).

28. Klein, J. P. Survival Analysis: Techniques for Censored and Truncated Data. New York, NY: Wiley, 1997. 
29. Hughes, L. A., Schouten, L. J., Goldbohm, R. A., van den Brandt, P. A. \& Weijenberg, M. P. Selfreported clothing size as a proxy measure for body size. Epidemiology 20,673-6 (2009).

30. May, A. M. et al. Effect of change in physical activity on body fatness over a 10 -y period in the Doetinchem Cohort Study. Am J Clin Nutr 92, 491-499 (2010).

31. Lynch, B. M. Sedentary behavior and cancer: a systematic review of the literature and proposed biological mechanisms. Cancer Epidemiol Biomarkers Prev 19, 2691-2709 (2010).

32. Lee, I. M., Paffenbarger, R. S., Jr \& Hsieh, C. Physical activity and risk of developing colorectal cancer among college alumni. J Natl Cancer Inst 83, 1324-1329 (1991).

33. Wolin, K. Y. et al. Change in physical activity and colon cancer incidence and mortality. Cancer Epidemiol Biomarkers Prev 19, 3000-3004 (2010).

34. Jass, J. R. Classification of colorectal cancer based on correlation of clinical, morphological and molecular features. Histopathology 50, 113-30 (2007).

35. McTiernan, A. et al. Effect of a 12-month exercise intervention on patterns of cellular proliferation in colonic crypts: a randomized controlled trial. Cancer Epidemiol Biomarkers Prev 15, 1588-1597 (2006).

36. Campbell, K. L. et al. Effect of a 12-month exercise intervention on the apoptotic regulating proteins
Bax and Bcl-2 in colon crypts: a randomized controlled trial. Cancer Epidemiol Biomarkers Prev 16, 1767-1774 (2007).

37. Abrahamson, P. E. et al. No effect of exercise on colon mucosal prostaglandin concentrations: a 12-month randomized controlled trial. Cancer Epidemiol Biomarkers Prev 16, 2351-2356 (2007).

38. Campbell, K. L. et al. No reduction in C-reactive protein following a 12-month randomized controlled trial of exercise in men and women. Cancer Epidemiol Biomarkers Prev 17, 1714-1718 (2008).

39. Siemiatycki, J. et al. Listing occupational carcinogens. Environ Health Perspect 112, 1447-1459 (2004).

40. Hughes, L. A. et al. Childhood and adolescent energy restriction and subsequent colorectal cancer risk: results from the Netherlands Cohort Study. Int J Epidemiol 39, 1333-1344 (2010).

41. Hughes, L. A. et al. Early life exposure to famine and colorectal cancer risk: a role for epigenetic mechanisms. PloS One 4, e7951 (2009).

42. Ferrari, P., Friedenreich, C. \& Matthews, C. E. The role of measurement error in estimating levels of physical activity. Am J Epidemiol 166, 832-840 (2007).

43. Baumgarten, M., Siemiatycki, J. \& Gibbs, G. W. Validity of work histories obtained by interview for epidemiologic purposes. Am J Epidemiol 118, 583-591 (1983). 


\section{4}

\section{Energy balance and unfavorable alleles in genes related to the IGF pathway jointly influence colon}

cancer risk in men

Colinda CJM Simons, Leo J Schouten, Roger WL Godschalk, Manon van Engeland, Piet A van den Brandt, Frederik-Jan van Schooten and Matty P Weijenberg

Ready for submission 


\begin{abstract}
-Abstract-
Background: We studied combined effects of energy balance-related colorectal cancer (CRC) risk factors and genetic variants in genes related to the insulin-like growth factor (IGF) pathway in relation to CRC risk. Our aim was to clarify the potential involvement of the IGF pathway in linking energy balance to CRC.
\end{abstract}

Methods: The Netherlands Cohort Study $(n=120,852)$ is characterized by a case-cohort approach. All participants completed a baseline questionnaire on diet and lifestyle, and $\sim 75 \%$ returned toenail clippings used for DNA isolation. We selected 25 SNPs in IGF pathway-related genes and an IGF1 19-CA repeat polymorphism from literature. Genetic variants were genotyped in DNA from subcohort members and CRC cases, totalling 6,234 samples after 16.3 years follow-up. With respect to SNPs, unfavorable alleles were aggregated into a literature-based genetic sum score. Categories of 8 energy balance-related CRC risk factors in combination with tertiles of the sum score and IGF1 19-CA repeat status were analyzed in relation to CRC risk by subsite in men and women.

Results: Twenty-four SNPs and the IGF1 CA repeat polymorphism were successfully genotyped. Eighteen SNPs, for which the literature was consistent on which allele was the unfavorable allele, were included in the sum score. Increasingly higher risks of proximal and distal colon cancer were observed in men with a larger trouser size, taller men (not for proximal colon cancer), and those who did not experience energy restriction in youth during the Dutch Hunger Winter (1944-45), when carrying more unfavorable alleles. Highest versus lowest combinations of categories showed hazard ratios $(95 \%$ confidence intervals, CI) of $1.85(1.15,2.99), 1.52(0.89$, $2.62)$, and $2.48(1.37,4.49)$ for proximal colon cancer, respectively, and hazard ratios $(95 \% \mathrm{CI})$ of $2.03(1.27,3.24), 2.05(1.16,3.63)$, and $1.87(1.11,3.16)$ for distal colon cancer, respectively. Combined effects were less clear in relation to rectal cancer and in women. Female (but not male) carriers of non-19/non-19 versus 19/19 IGF1 CA repeats were at a decreased proximal and distal colon cancer risk, independent of energy balance-related CRC risk factors.

Conclusion: Combined effects of energy balance-related CRC risk factors and IGF pathwayrelated SNPs influenced colon cancer development in men. Associations might have been absent in women due to effects of estrogen-related factors, counteracting insulin resistance. The strength of this study is that joint effects were evident for multiple energy balance-related CRC risk factors in the hypothesized direction. 


\subsection{Introduction}

Colorectal cancer (CRC) has emerged as a complex disease as indicated by that risk factors differ in men and women and for cancers in different subsites. ${ }^{1-3}$ Indicators of energy balance such as body fatness and physical activity are established risk factors that show heterogeneous associations with CRC risk. ${ }^{4,5}$ Elucidation of the heterogeneity in associations may be achieved by investigating pathways that link these factors to CRC risk. The study of gene-environment ( $\mathrm{G} \times \mathrm{E}$ ) interactions will help do so. ${ }^{6}$

The availability of extensive data on energy balance-related exposures is important because, in the absence of a statistically significant test for interaction, repeated significant combined effects in the hypothesized direction may still indicate the involvement of the pathway of interest. It has been described that a four times larger sample size is required to detect an interaction effect as compared to a marginal effect of similar magnitude. ${ }^{6}$ As a result, especially if effects are small, the power to detect a statistically significant interaction may be limited, 7 even in what appear to be large studies. Statistical tests for interaction also inherently assume the presence of main effects because these tests assess whether the observed combined effect differs from the expected combined effect; however, main effects need not be present. ${ }^{7,8}$

We investigated combined effects of 8 energy balance-related CRC risk factors and genetic variants in genes related to the insulin-like growth factor (IGF) pathway within the Netherlands Cohort Study (NLCS). The IGF pathway is central to regulating growth in the body and is a putative link between energy balance and CRC risk. The evidence for this includes: 1 ) obesity has been found to increase IGF-1 levels and decrease levels of IGF binding proteins (IGFBPs), particularly IGFBP-1 and -2 , whereas energy restriction and prolonged exercise have been found to accomplish the opposite of these effects; ${ }^{9} 2$ ) higher levels of IGF-1 and lower levels of IGFBPs have been associated with an increased CRC risk;9-12 and 3) type 2 diabetics have been found to be at an increased risk of CRC, which is likely explained by hyperinsulinaemia stimulating the production of IGF-1. ${ }^{13,14}$ The NLCS enabled the estimation of sex- and subsite-specific CRC risks. SNPs were studied in a cumulative fashion by aggregating the number of unfavorable alleles into a literature-based genetic sum score, representing an individual's 'at-risk' genetic background. This approach was chosen because most single SNPs have been observed to confer only minor effects ${ }^{15}$ and because gene-gene interactions ${ }^{16}$ and functional compensation between genes are likely. ${ }^{17}$

\subsection{Methods}

\subsubsection{Study population and design}

The Netherlands Cohort Study (NLCS) includes 120,852 men and women who were between 55-69 years old at baseline in 1986, when completing a self-administered questionnaire on diet and cancer. ${ }^{18}$ The baseline questionnaire included a semi-quantitative 150-item food frequency questionnaire (FFQ), which was found to rank individuals adequately according to dietary intake when compared to a 9-day dietary record, ${ }^{19}$ and was shown a good indicator of intake for at least 5 years. ${ }^{20}$ Nutrient intakes were calculated on the basis of the Dutch food composition table 1986-87. ${ }^{21}$ Participants who reported a history of cancer (other than skin cancer) were excluded from follow-up. Along with returning the questionnaire, participants were asked to return toenail clippings by way of an enclosed envelope that had to be sealed. Approximately 90,000 participants provided toenail clippings, which turned out to be a valid DNA 
source for the genotyping of germline genetic variants. ${ }^{22}$ The NLCS and the use of toenail DNA was approved by the review boards of the TNO Nutrition and Food Research Institute (Zeist, the Netherlands) and Maastricht University (Maastricht, the Netherlands).

The NLCS is characterized by a case-cohort design. This design entails that a random subcohort $(n=5,000)$, selected immediately after baseline, is followed up to estimate the accumulated person-time at risk, whereas incident cancer cases are enumerated for the entire cohort. Follow-up for vital status is performed through linkage to the Central Bureau of Genealogy and the municipal population registries ( $100 \%$ completeness). Cancer follow-up is performed through linkage with the population-based cancer registry and PALGA (the Netherlands pathology database) (more than 96\% completeness). ${ }^{23,24}$ After 16.3 years, there were 4,774 subcohort members and 3,440 incident CRC cases. Toenail clippings were available for 3,768 subcohort members (78.9\%) and 2,580 CRC cases (75.0\%); 114 subcohort members who developed CRC during followup were included in both counts, leaving a total of 6,234 unique toenail samples.

\subsubsection{Energy balance-related exposures}

Exposures related to energy balance were derived from the baseline questionnaire. Energy balance denotes the ability to maintain a stable body weight via balancing energy intake with energy expenditure.

Available adult life exposures indicative of body fatness were body mass index (BMI, $\mathrm{kg} / \mathrm{m}^{2}$ ), and trouser/skirt size. Trouser/skirt size was shown to correlate well with hip and waist circumferences in a subset of weightstable NLCS participants, and was associated with endometrial and renal cell cancer risk in a fashion as would be expected for waist cir- cumference. ${ }^{25}$ When adjusted for BMI, waist circumference is thought to reflect abdominal fatness. ${ }^{26,27}$

Available early life exposures were BMI at age $20\left(\mathrm{~kg} / \mathrm{m}^{2}\right)$, height $(\mathrm{cm})$ - which reflects exposure to growth factors during childhood - and early life energy restriction - which likely induced a negative energy balance-as measured through three proxy variables. The proxies used for energy restriction were the place of residence during the Hunger Winter (1944$45)$, the place of residence during the War Years (1940-44), and whether or not an individual's father was employed during the Economic Depression (1932-40). Nutritional differences between individuals during these periods have been described in detail. In particular living in a Western city in the Netherlands during the Hunger Winter indicated severe energy restriction. During this winter, official daily rations per capita were between $400-800$ kilocalories, although the diet remained nutritionally balanced. ${ }^{28,29}$

A healthy energy balance may be maintained through engaging in physical activity. In the NLCS, occupational physical activity in men and non-occupational physical activity in women were indicative of long-term physical activity (the number of long-term employed women was low).$^{30}$ Information on occupational physical activity was derived from an individual's self-reported longest held job. The categorization used distinguished between jobs in which there was an occupational energy expenditure of $<8,8-12$, and $>12$ $\mathrm{kJ} / \mathrm{min} .{ }^{31}$ Non-occupational physical activity in minutes per day was a sum measure of several activities: daily walking/cycling (min/day), and weekly recreational walking/cycling, weekly engagement in gardening/doing odd jobs, and weekly participation in sports/gymnastics (categories: never, 1, 12 , and $>2$ hours/week). 


\subsubsection{Toenail DNA isolation}

DNA isolation from toenail clippings was performed according to the DNA extraction protocol of Cline et al. ${ }^{32}$ with some adjustments. ${ }^{22}$ All procedures were carried out at room temperature, unless stated otherwise. In short, the first step was to soak approximately $15 \mathrm{mg}$ of toenail clippings (the optimal amount for isolation) into acetone for $10 \mathrm{~min}$ to remove any nail polish. This acetone treatment was repeated once, after which the clippings were allowed to dry. Possible exogenous material was removed by incubating the clippings in $200 \mu \mathrm{L} 1 \%$ SDS/ $25 \mathrm{mM}$ EDTA and $1 \mu \mathrm{L} 20$ $\mathrm{mg} / \mathrm{mL}$ proteinase $\mathrm{K}$ for $1 \mathrm{~h}$. The clippings were then rinsed 3 times in MilliQ and subsequently incubated in $200 \mu \mathrm{L} 2 \mathrm{~N} \mathrm{NaOH}$ on an automated vortex $\mathrm{o} / \mathrm{n}$ to solubilize the clippings. The solution was neutralized by adding $100 \mu \mathrm{L} 200 \mathrm{mM}$ Tris- $\mathrm{HCl} \mathrm{pH} 7.4$ and $34.5 \mu \mathrm{L}$ $11.6 \mathrm{~N} \mathrm{HCl}$. In the case that a precipitate was formed, diluted $\mathrm{NaOH}$ was added to redissolve the precipitate. Next, an equal volume of phenol/chloroform was added to the neutralized sample, mixed for $5 \mathrm{~min}$ by inversion and centrifuged at maximum speed (21,000 relative centrifugal force) for $5 \mathrm{~min}$. The aqueous top-layer was transferred to a clean tube and DNA was precipitated by adding $1 / 10$ volume of $3 \mathrm{M}$ sodium acetate $\mathrm{pH}$ $5.2,2$ volumes of $95 \%$ ethanol, and $1 \mu \mathrm{L} 20$ $\mathrm{mg} / \mathrm{mL}$ glycogen $\mathrm{o} / \mathrm{n}$ at $-20^{\circ} \mathrm{C}$. DNA was pelleted by centrifugation at maximum speed for $30 \mathrm{~min}$ at $4^{\circ} \mathrm{C}$, washed once in $75 \%$ ethanol, dried, and resuspended in $25 \mu \mathrm{L} 2 \mathrm{mM}$ Tris$\mathrm{HCl}$. The resulting toenail DNA solution was stored at $-30^{\circ} \mathrm{C}$ until it was used for genotyping. The average DNA yield of $15 \mathrm{mg}$ of toenail clippings was $4.7 \mu \mathrm{g}$ (SD: 8.9). Isolated toenail DNA was fragmented ( $<200$ bp on average) and showed some organic and protein contamination, requiring that its valid use on a specific genotyping platform is tested prior to proceeding to high-throughput genotyping.

\subsubsection{SNP genotyping}

SNP genotyping was performed on the SEQUENOM ${ }^{\circledR}$ MassARRAY ${ }^{\circledR}$ platform using the iPLEX ${ }^{\mathrm{TM}}$ assay (Sequenom Inc., Hamburg, Germany). This platform allows highthroughput genotyping of up to 40 SNPs at once. Toenail DNA tested suitable for use on this platform in a pilot study, showing high SNP call rates (mean: $96.8 \%$ ), sample call rates (mean: $99.0 \%$ ), and a high reproducibility (range: $98.9 \%-100 \%$ ) as based on 89 quadruple samples genotyped for 30 SNPs using a single assay. Extension primers for SNPs in the pilot study and in the current study were designed using MassARRAY ${ }^{\circledR}$ software, version 4.0. Polymerase chain reaction (PCR) amplification and extension reactions were performed according to the manufacturer's instructions. Extension product sizes were determined by mass spectrometry with the use of the iPLEX Gold system. The protocol is publicly available. ${ }^{33}$ Of importance, genotyping was performed using $100 \mathrm{ng}$ of toenail DNA, which was pipetted on to 384-well plates. A duplicate sample was included for a random selection of $\sim 5 \%$ of the samples $(\mathrm{n}=$ 314 ). In addition, 436 water controls were included, dispersed across the plates.

\subsubsection{Gene and SNP selection}

Our gene and SNP selection strategy was literature-based. We selected genes encoding for the following factors in the IGF pathway: IGF1 and -2 , their receptors and receptor substrates, IGFBP-1 through 7, IGFBP acid labile subunit, growth hormone 1 , and growth hormone-regulating factors. With respect to the IGF pathway, IGF-1 and -2 are main growth factors during adult life and gestation, respectively. IGFBPs bind to IGFs, generally inhibiting growth by preventing IGFs from reaching target tissues. The majority of IGFs $(\sim 90 \%)$ is bound to IGFBP-3. IGF-1 and IGFBPs are mainly produced in the liver under stimula- 
tion of growth hormone 1, although expression of IGFs and IGFBP-2-7 has also shown present in cells in the colorectum. ${ }^{9,10,34}$ IGF-1 and IGFBPs can form a ternary complex with IGFBP acid labile subunit, extending the halflife of IGF-1. ${ }^{35}$ Free IGFs are able to bind to the IGF-1 receptor. Upon binding of IGFs to the IGF-1 receptor, insulin receptor substrates become phosphorylated, making them the docking station for various proteins, and thereby influencing multiple downstream signalling pathways. The IGF-2 receptor mainly acts as a clearance receptor for IGF-2.10,36,37
Apart from genes directly involved in the IGF pathway, we also identified genes encoding for factors secreted by adipose tissue. These adipokines were adiponectin, its receptors, and the peroxisome proliferator-activated receptor gamma. All have been associated with glucose and lipid homeostasis, insulin resistance and compensatory hyperinsulinaemia. 13,38,39 The complete set of the 22 selected genes and their interrelationships are depicted in Figure 1.

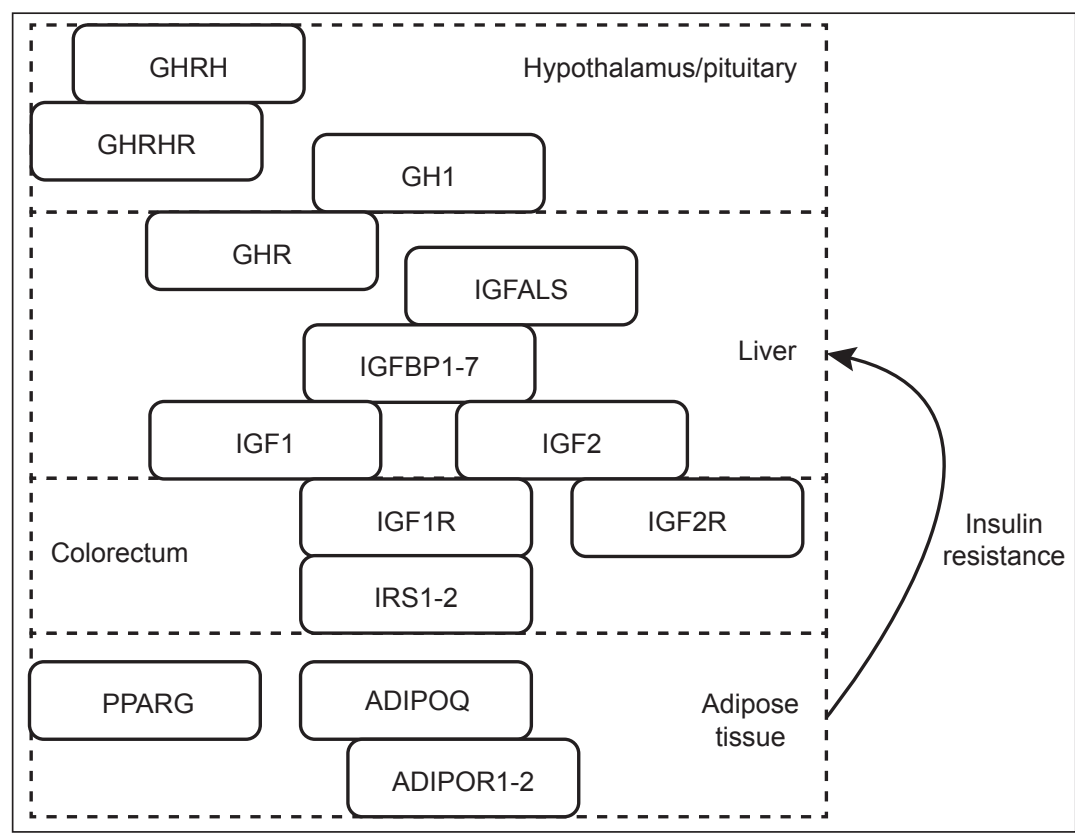

Figure 1. Genes related to the insulin-like growth factor pathway selected for study. Touching boxes indicate the binding of gene products to other gene products.

\begin{abstract}
Abbreviations: ADIPOQ, adiponectin; ADIPOR1-2, adiponectin receptor 1 and 2; $\mathrm{GH} 1$, growth hormone 1; GHR, growth hormone receptor; GHRH, growth hormone releasing hormone; GHRHR, growth hormone releasing hormone receptor; IGF1-2, insulin-like growth factor 1 and 2, IGFALS, insulin-like growth factor binding protein acid labile subunit; IGFBP17 , insulin-like growth factor binding protein 1 through 7; IGF1-2R, insulin-like growth factor 1 and 2 receptor; IRS1-2, insulin receptor substrate 1 and 2; PPARG, peroxisome proliferator-activated receptor gamma.
\end{abstract}

Our SNP selection strategy is shown in Figure 2. Four steps can be distinguished. In step 1, we searched the literature for SNPs in the selected genes that were associated with i) the risk of $\mathrm{CRC}$, ii) traits of interest, i.e. obesity, insulin resistance or blood levels of IGF pathway-related factors, iii) the risk of type 2 dia- betes mellitus, and iv) the risk of other obesity-related cancers. We also specifically searched for GWAS hits in these genes in relation to v) CRC, vi) type 2 diabetes mellitus, and vii) traits of interest. The search was concluded in March 2012. For power considerations, only SNPs with more than a $10 \%$ pre- 
valence of the rare homozygous and heterozygous genotypes were taken forward to step 2.

In step 2, SNPs were scored on each of the seven points (i-vii) described according to the level of evidence. If no association was reported in the literature with respect to the specific endpoint, SNPs were given a score of ' 0 ' on that point; SNPs were scored ' 1 ' if found associated once; and SNPs were scored ' 2 ' if found associated in two or more studies.

In step 3, the total score across points i-vii was used to rank SNPs. SNPs associated with several endpoints - and thus a higher sum score - were thought to more likely influence CRC risk in combination with indicators of energy balance. In order to minimize the chance of selecting SNPs based on falsepositive results, SNPs with a sum score of less than two, with the exception of missense SNPs, were excluded.

Step 4 concerned the assay design. Here, we designated the 20 highest ranked SNPs as high-priority SNPs (all had a sum score higher than 2), because multiplexing is often not $100 \%$ efficient due to sequence incompatibilities between the sequences flanking the SNPs. The remaining low-priority SNPs were used for 'superplexing', i.e. given a fixed, optimal design as based on the set of high-priority SNPs, these SNPs were added to the design if possible. In total, 25 SNPs in 9 genes were included in the final genotyping assay.

\subsubsection{IGF1 CA repeat}

Several studies have hypothesized the potential importance of the IGF1 19-CA repeat polymorphism in CRC. Therefore, we also included this variant and genotyped it by PCR amplification and subsequent analysis of the PCR products' length using the 96-capillary ABI 3730xl DNA Analyzer. The PCR was car- ried out using $100 \mathrm{ng}$ of DNA, $10.75 \mu \mathrm{l}$ MilliQ, $2.5 \mu \mathrm{l} 10 \mathrm{x}$ PCR buffer, $0.875 \mu \mathrm{l}$ of $50 \mathrm{mM}$ MgCL2, 2x $0.125 \mu$ l of Primer predilution-mix (10 times diluted), $0.5 \mu \mathrm{l}$ of $10 \mathrm{mM}$ dNTP mix, and $0.125 \mu \mathrm{l}$ of Platinum Taq Polymerase (Life Technologies, Bleiswijk, the Netherlands). The primers (forward: 5'-ACCACTCTGGGAGAAGGGTA-3'; reverse: 5'-GCTAGCCAGCTGGTGTTATT-3') were fluorescently labelled with 6-FAM (blue), NED (yellow), and PET (red), which enabled the simultaneous analysis of three samples in a single 96-well. The protocol was carried out in the dark due to the lightsensitivity of the fluorescent labels. The PCR reactions were performed using the following cycles: $94^{\circ} \mathrm{C}$ for $10 \mathrm{~min}$, followed by 35 cycles of $94^{\circ} \mathrm{C}$ for $30 \mathrm{sec}, 55^{\circ} \mathrm{C}$ for $30 \mathrm{sec}$, and $72^{\circ} \mathrm{C}$ for $30 \mathrm{sec}$, followed by $72^{\circ} \mathrm{C}$ for $10 \mathrm{~min}$ and $4^{\circ} \mathrm{C}$ for $30 \mathrm{~min}$. The analysis included $314 \mathrm{dupli}-$ cate samples and 436 water controls. In line with previous studies, ${ }^{40-48}$ individuals were categorized as homozygous for the wild type allele (19/19), heterozygous with one wild type and one variant allele (19/non-19) or homozygous for variant alleles (non-19/non19).

\subsubsection{Statistical analysis}

Genotyping results were evaluated as to sample call rates, SNP call rates, reproducibility, allele frequencies, and deviations from Hardy-Weinberg Equilibrium (using Pearson $\chi^{2-}$ tests). To evaluate SNP-disease associations, a literature-based genetic sum score was created by aggregating the number of unfavorable alleles in each individual. For instance, in a twoSNP example, an individual heterozygous for one SNP and homozygous for the unfavorable allele on the other SNP would receive a sum score of 3. Alleles were considered 'unfavorable' if associated with CRC endpoints, type 2 diabetes mellitus or other obesity-related cancers in a risk-increasing manner, or if associated with overweight, obesity, insulin resistance or blood levels of IGF pathway-related factors 


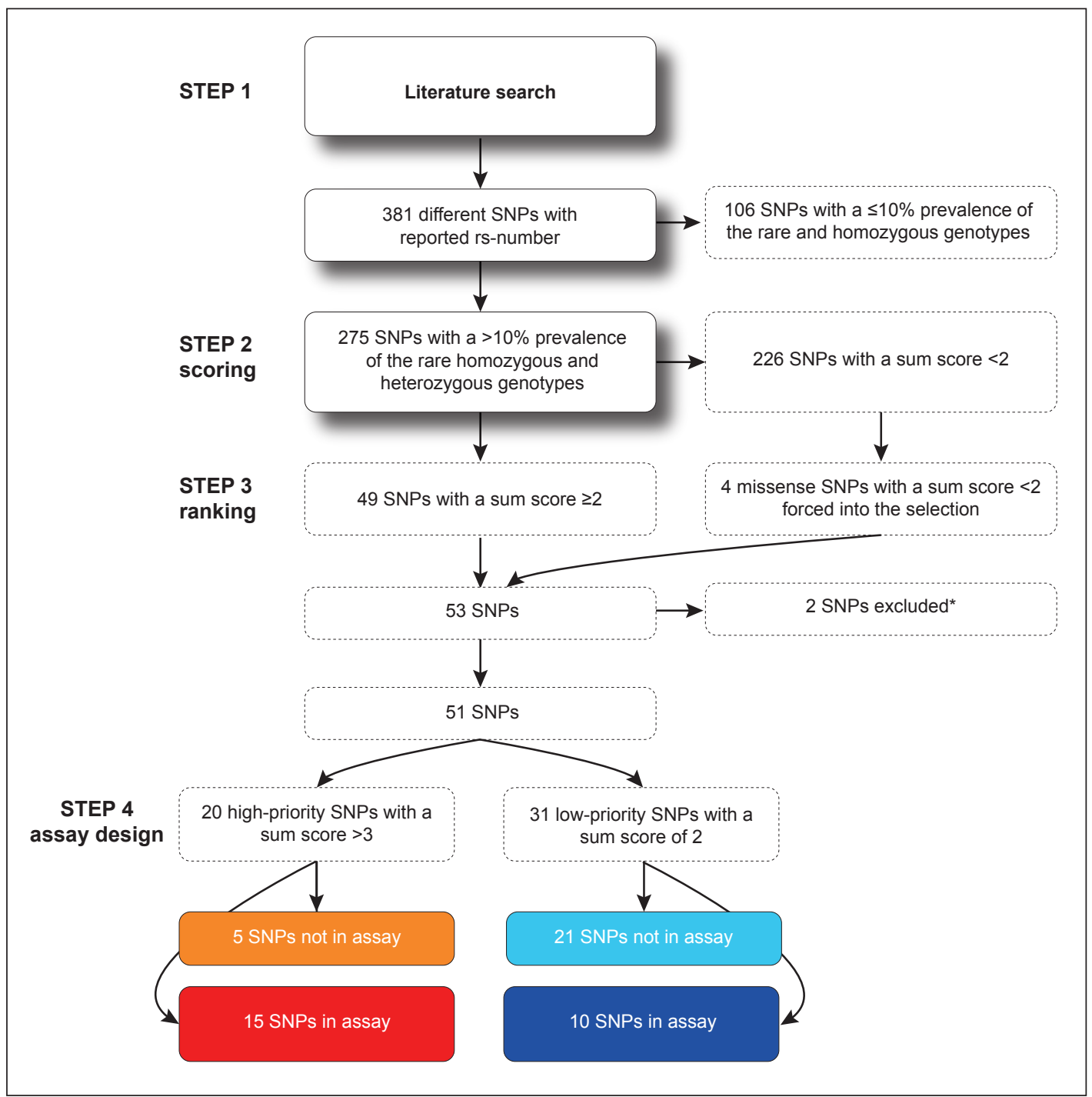

Figure 2. SNP selection strategy.

* One SNP was excluded because of complete linkage disequilibrium (LD) with another SNP ( $\left.\mathrm{r}^{2}=1\right)$; a second SNP was excluded because it failed in a pilot and no proxy was available. Another SNP that failed in the pilot could be replaced by a proxy SNP in complete LD with the original SNP.

in a manner that may increase CRC risk. The literature-based genetic sum score was categorized into tertiles to enable tests for trend, while maintaining optimal power.
In previous molecular epidemiological studies, we have shown a relationship between a genetic sum score and biomarkers of carcinogenesis. ${ }^{49,50}$ Here, sex- and subsite-specific hazard ratios and $95 \%$ confidence intervals for $\mathrm{CRC}$ were estimated in relation to the litera- 
ture-based genetic sum score and in relation to the IGF1 CA repeat using an age-adjusted Cox model. For literature comparison, potential single SNP effects on the sex- and subsitespecific risk of CRC were analyzed assuming an additive inheritance model.

To investigate combined effects, Cox regression was used to estimate hazard ratios for linear combinations of tertiles of the literaturebased genetic sum score and categories of the following 8 factors related to energy balance: adult BMI, adult trouser/skirt size, BMI at age 20 , height, early life energy restriction as measured through three proxy variables, and physical activity. Adult BMI, BMI at age 20, and height were categorized into sex-specific tertiles for power considerations. Analyses were performed using age-adjusted and multivariable-adjusted models. The latter included the predefined potential confounders: age (years), smoking status (never, ex, current), alcohol intake $(0,0.1-29, \geq 30 \mathrm{~g} /$ day $)$, total energy intake ( $\mathrm{kcal} /$ day), processed meat intake (g/day), meat intake (g/day), and a firstdegree family history of CRC (yes or no). In addition, all multivariable-adjusted models, except models for (non-)occupational physical activity, were adjusted for (non-)occupational physical activity; models for adult trouser/skirt size, (non-)occupational physical activity, and early life energy restriction were adjusted for adult BMI $\left(\mathrm{kg} / \mathrm{m}^{2}\right)$; and models for height were adjusted for adult weight $(\mathrm{kg})$. Analyses of combined effects of energy balance-related factors and the IGF1 CA repeat were performed in the same manner.

To account for the additional variance introduced by sampling the subcohort from the entire cohort, standard errors were estimated using the robust Huber-White sandwich estimator. ${ }^{51}$ The proportional hazards assumption was tested using the scaled Schoenfeld residuals ${ }^{52}$ and by visually inspecting the -log-log- transformed hazard curves (there were no apparent violations).

Multiplicative interactions were tested using the Wald test. We also calculated the relative excess risk due to interaction (RERI) ${ }^{53}$ to assess departure from additivity of effects on a hazard ratio scale. The RERI was derived by applying the formula $R E R I=R R_{11}-R R_{10}-$ $R R_{01}+1$ to the hazard ratios derived from the Cox models, and corresponding 95\% biascorrected confidence intervals were estimated by bootstrapping ( $\mathrm{n}$ bootstrap samples $=$ 1,000). ${ }^{54}$ As the RERI was developed for risk factors, we recoded the reference category to that hypothesized to confer the highest protective effect when genes and environment were considered jointly in models for early life energy restriction and physical activity, as suggested by Knol et al. ${ }^{55}$

All analyses were conducted using Stata version 12 (Stata Corp., College Station, TX). Graphical plots were produced using $R$ version 2.15.1 (the R Foundation for Statistical Computing). To check for the influence of preclinical disease in the combined effects analyses, sensitivity analyses were conducted in which the first two years of follow-up were excluded. These analyses are not presented because the interpretation of results was unchanged. Statistical significance was indicated by a $P$-value $<0.05$ for two-sided testing.

\subsection{Results}

\subsubsection{Laboratory analyses}

SNPs. Twenty-four of the 25 SNPs in IGF pathway-related genes were successfully genotyped in 6,230 toenail DNA samples and 311 duplicate samples of subcohort members and CRC cases. The IGF1 SNP rs35767 was excluded because it only showed calls for the C allele due to self-extension of the primer. The median sample call rate in samples was 100\% 
(mean: 95.4\%). The median SNP call rate was 96.1\% (range: $83.6 \%-97.9 \%$ ). The reproducibility of the 24 successfully analyzed SNPs in duplo pairs ranged from $98.8 \%-100 \%$ between SNPs, as based on duplo pairs with successful analyses for the SNP in question (n ranged from 243-303). One duplo pair was excluded from further analysis because of highly irreproducible results. Exclusion of samples with a SNP call rate $<95 \%$ resulted in the exclusion of another 532 samples (8.5\%), leaving 5,697 samples. All SNPs adhered to Hardy Weinberg Equilibrium (HWE) in subcohort members, except SNP rs1342387. Table 1 shows the genotype and allele frequencies per SNP in subcohort members, and shows that for 18 SNPs a decision could be made with respect to which allele was the unfavorable allele on the basis of the literature. These SNPs were used to generate the literature-based genetic sum score.

IGF1 19-CA repeat. Genotyping of the IGF1 19CA repeat yielded a sample call rate of $70.7 \%$ in 6,230 samples. The reproducibility of the IGF1 CA repeat was $93.6 \%$, as based on duplo pairs with successful analyses $(n=202)$. The 19/19, 19/non-19, and non-19/non-19 genotypes occurred in 707 (33.1\%), 818 (38.3\%), and $609(28.5 \%)$ subcohort members, respectively. The IGF1 CA repeat was not in $\operatorname{HWE}(P$ $<0.001$ ).

\subsubsection{Baseline characteristics}

Participants with inconsistent or incomplete baseline questionnaires were excluded from the analysis, leaving a total of 3,203 subcohort members and 2,274 CRC cases with available genotyping data. Of these, 134 subcohort members and 120 CRC cases could not be classified into one of the tertiles of the literature-based genetic sum score because of uncertainty about which tertile the person belonged to due to missing data (one SNP per individual was missing at most). Tertiles of the genetic sum score ranged from 6-14, 1518, and 19-29 unfavorable alleles, respectively. The theoretical maximum was 36 .

Comparison of energy balance-related exposures and potential confounders between subcohort members and CRC cases most clearly showed a difference in the percentage of individuals with a family history of CRC (Table 2). To check for bias due to the potential selective return of toenail clippings, the baseline characteristics were also compared between subcohort members who returned toenail clippings and those who did not; there were no essential differences (data not shown).

\subsubsection{Marginal associations}

NLCS data on marginal associations between energy balance-related exposures and CRC risk were published previously. In short, these data indicated that overweight in men particularly as indicated by a larger trouser size - and height in women were risk factors for distal colon cancer, ${ }^{56}$ whereas occupational physical activity in men and non-occupational physical activity in women were protective against distal colon cancer and cancer in all colorectal subsites, respectively..$^{30}$ Furthermore, early life energy restriction decreased the risk of proximal colon and rectal cancer in men and the risk of rectal cancer in women. ${ }^{57}$

With respect to the literature-based genetic sum score, men in the highest versus lowest tertile, i.e. those having 19 or more versus 6-14 unfavorable alleles, were at a $36 \%$ increased risk of overall CRC (95\% confidence interval (CI): $1.11,1.65$; $P$-trend $=0.002)$, a $34 \%$ increased risk of proximal cancer (95\% CI: 1.00, $1.79 ; P$-trend $=0.06)$, and a $48 \%$ increased risk of distal colon cancer (95\% CI: 1.12, 1.94; $P$ trend $=0.006)$; there was no association with rectal cancer risk (highest versus lowest tertile: hazard ratio $(\mathrm{HR})=1.17,95 \% \mathrm{CI}: 0.86$, 1.60; $P$-trend $=0.25$ ) (Table 3 ). In women, no 


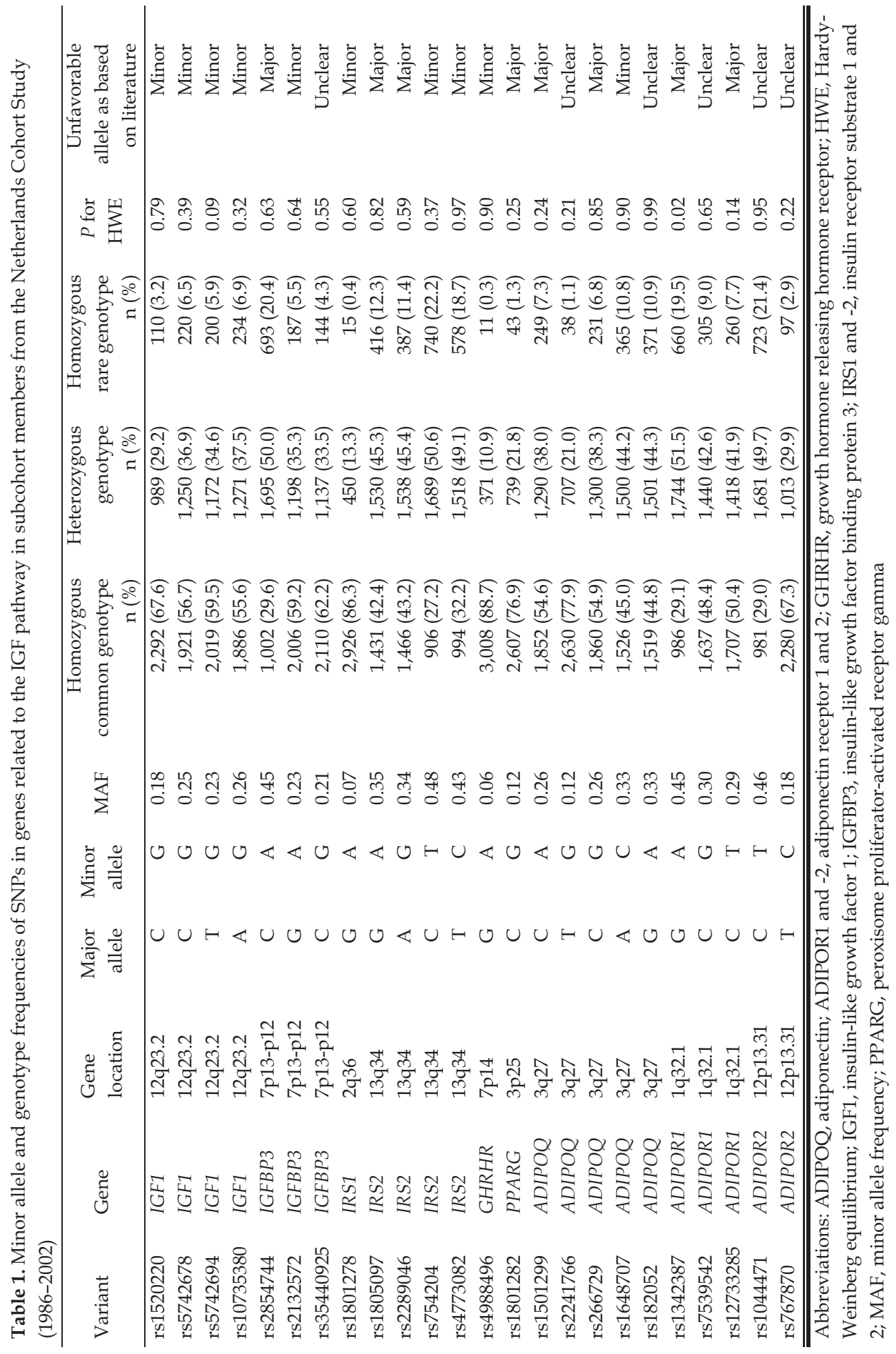




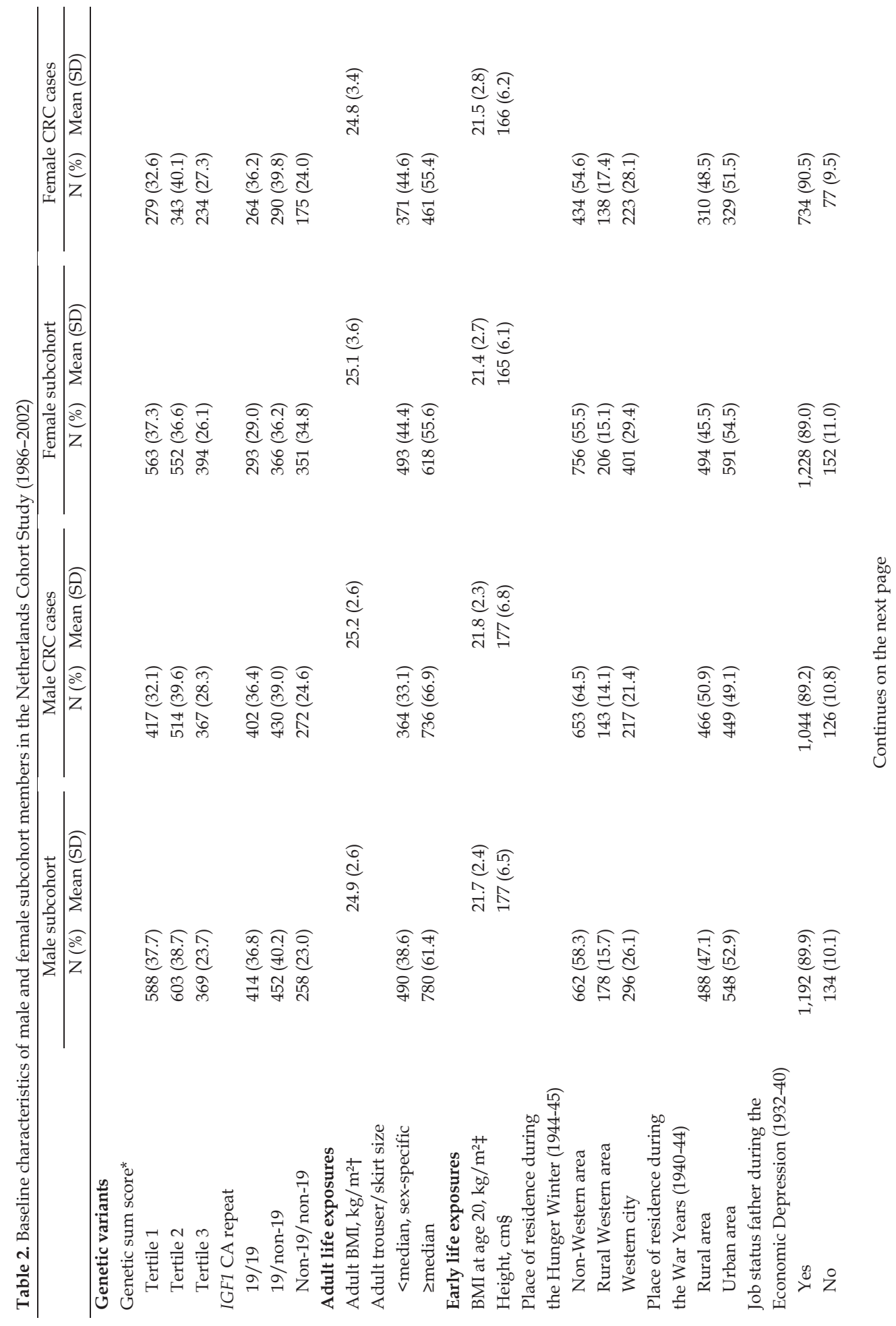


กิ 6 वृ वे ब्व तปสำ 웜

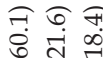

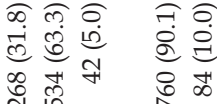

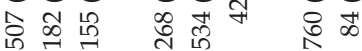

อิ สิ वे लुं व्य

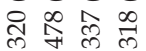

그들 进 o c 용

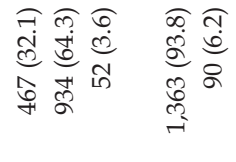

เ઼

๓ $\infty$

กุ่ กู่ กุ

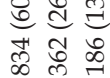

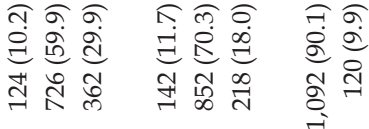

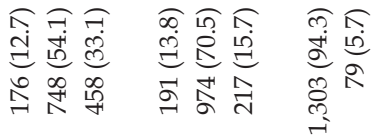

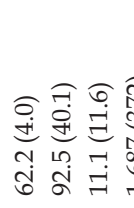

Ũ

(

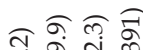
पँ

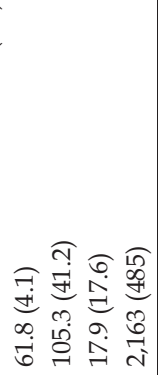

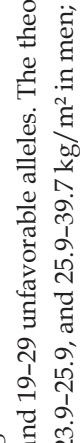

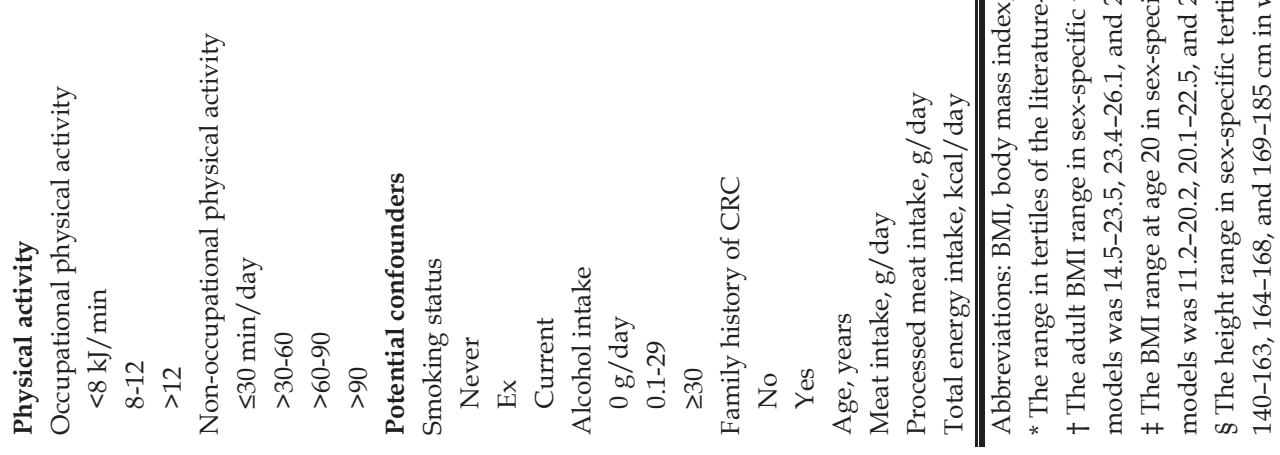


significant associations were observed, although there was a borderline significant trend towards an increased rectal cancer risk for those in higher tertiles of the genetic sum score (middle and highest versus lowest tertile: $\mathrm{HR}=1.28,95 \%$ CI: $0.85,1.91$ and $\mathrm{HR}=$ 1.50, 95\% CI: $0.98,2.28$, respectively; $P$-trend $=$ $0.06)$.

The IGF1 CA repeat was not associated with $\mathrm{CRC}$ risk in men, yet was associated with a significant decrease in proximal and distal colon cancer risk in women homozygous for variant alleles versus those homozygous for the wild type allele (19 repeats) (HR for CRC $=0.54,95 \%$ CI: $0.42,0.70$; $P$-trend < 0.001) $(\mathrm{Ta}$ ble 3). Single SNP-CRC associations were generally not significant, though indicative of potentially increased risks. Associations are shown in Supplemental Table 1.

\subsubsection{Combined effects of $\mathrm{G}$ \& E}

Linear combinations of categories of energy balance-related factors and tertiles of the literature-based genetic sum score showed differential results in men and women. Although the multiplicative interactions and estimated relative excess risks due to interaction in both men and women were generally not statistically significant (Supplemental Tables 2 and $3)$, associations in men were repeatedly in the hypothesized direction (Figure 3; Supplemental Table 2).

Men in the highest tertile of the genetic sum score who had an equal to or above median adult trouser size were at a significantly increased risk of overall CRC, specifically proximal and distal colon tumors, as compared with those in the lowest category of combined exposures (HR for proximal colon cancer = 1.85, 95\% CI: 1.15, 2.99; HR for distal colon cancer $=2.03,95 \%$ CI: 1.27, 3.24) (Figure 3; Supplemental Table 2). This association did not exist with respect to rectal cancer. Adult
BMI, when considered in combination with the genetic sum score, was significantly associated with CRC risk and borderline significantly associated with proximal colon cancer risk in men (HR $=1.55,95 \% \mathrm{CI}: 1.06,2.25$; and $\mathrm{HR}=1.67,95 \% \mathrm{CI}: 0.99,2.83$, respectively); there were no associations with distal colon and rectal cancer risk (Figure 3; Supplemental Table 2).

With respect to early life exposures in men, $\mathrm{BMI}$ at age 20 was not associated with CRC endpoints when considered in conjunction with the genetic sum score. Men, who were among the tallest individuals in this study, and who were in the highest tertile of the genetic sum score, were at a significantly increased risk of CRC, particularly distal colon tumors (HR $=2.05,95 \%$ CI: 1.16, 3.63) (Figure 3; Supplemental Table 2). Data on early life energy restriction during the Hunger Winter showed that the absence of energy restriction conferred strongly increased risks of proximal and distal colon cancer in men in the highest tertile of the genetic sum score; the comparison group consisted of men who had experienced energy restriction and who were in the lowest tertile of this sum score. Statistically significant hazard ratios were between 1.87 and 2.48. Models for early life energy restriction during the War Years, but not during the Economic Depression, supported the combined effects in relation to the risk of proximal colon cancer. Rectal cancer risk appeared to be influenced by early life energy restriction during the Hunger Winter independently of the genetic sum score (Figure 3; Supplemental Table 2).

With respect to occupational physical activity in men, the highest category of combined exposures, reflecting a low level of physical activity and a high genetic sum score, was associated with a significantly increased distal colon cancer risk. However, there was also a significant main effect of the genetic sum 


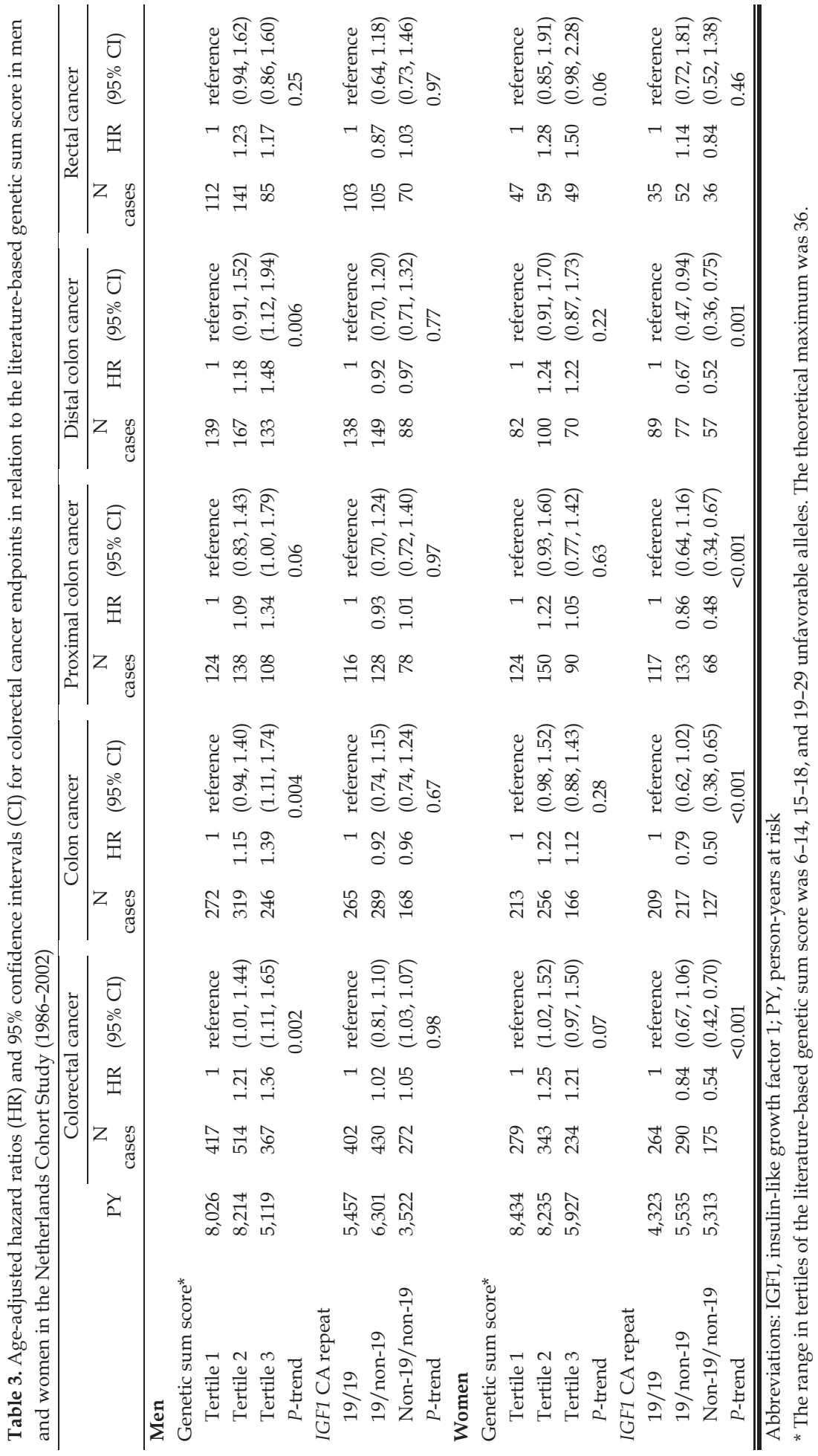



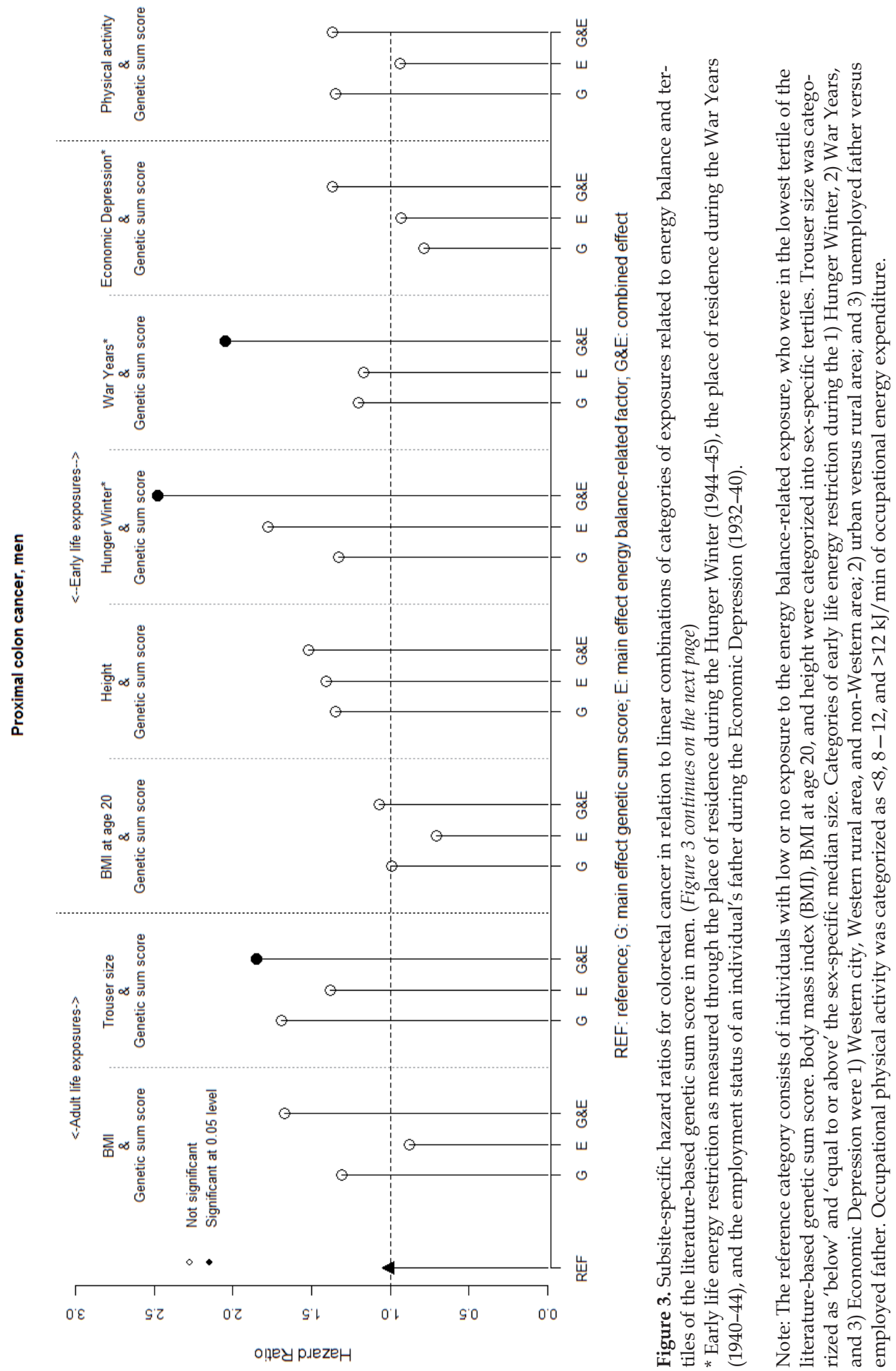

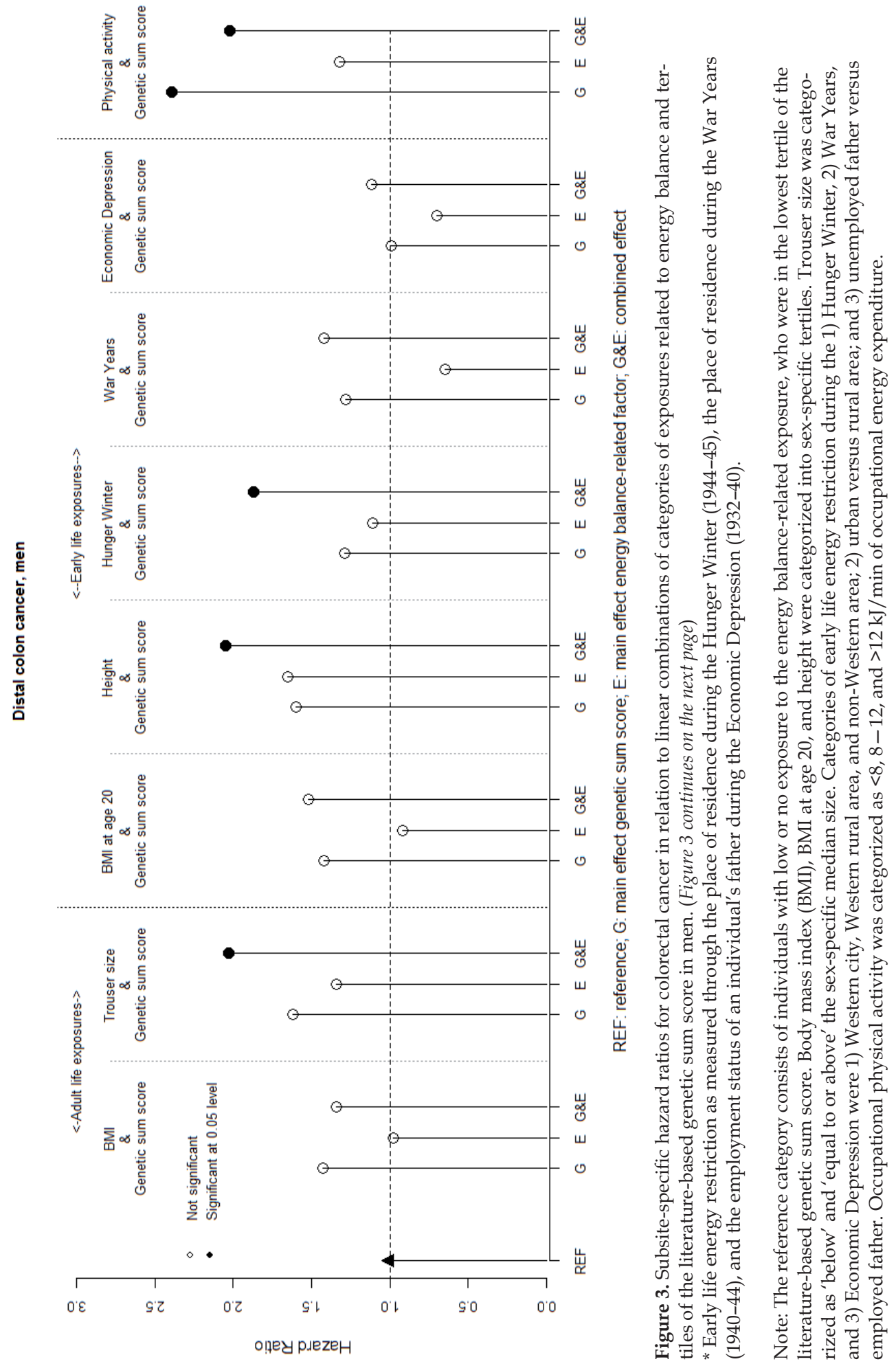

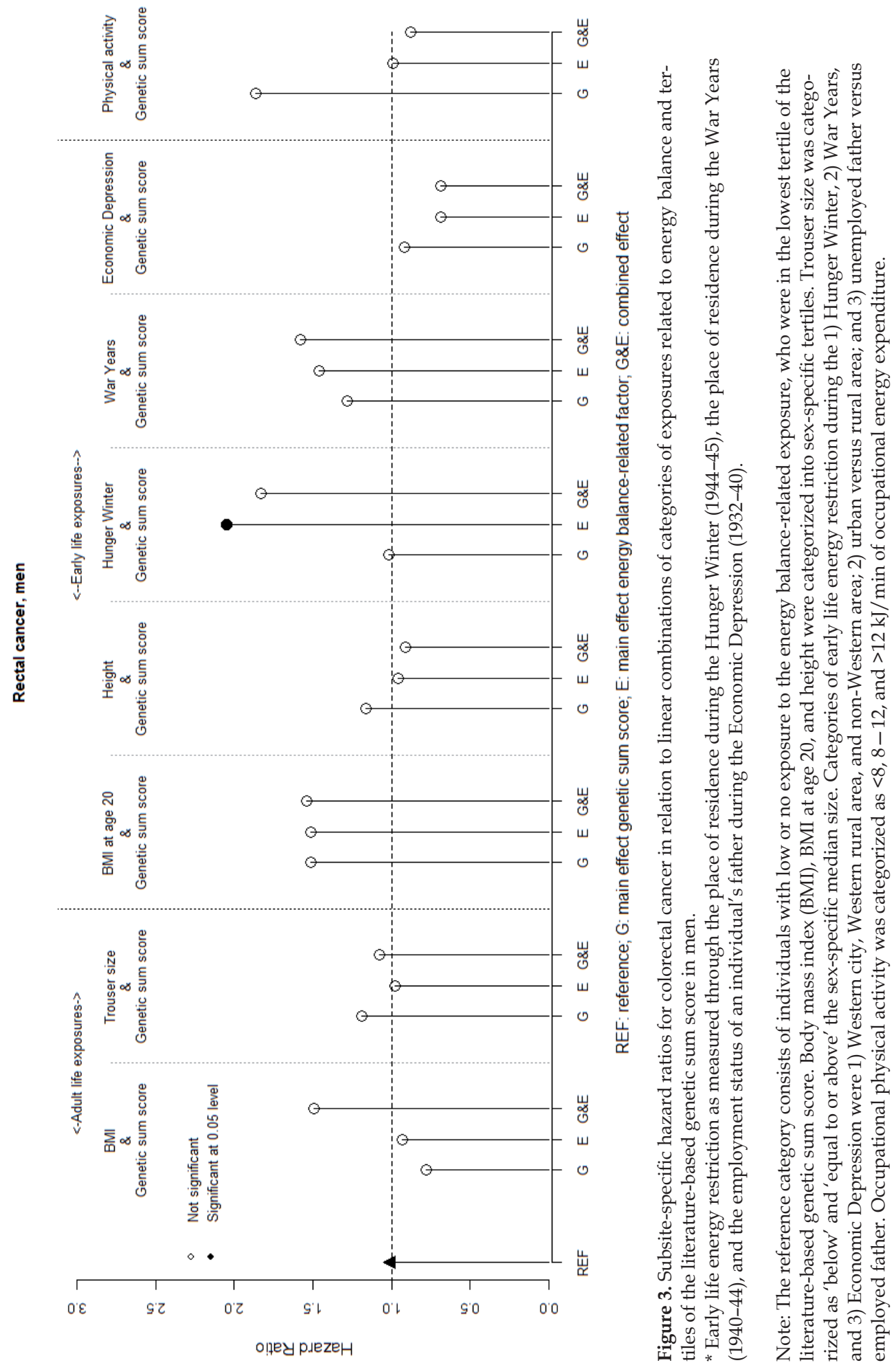

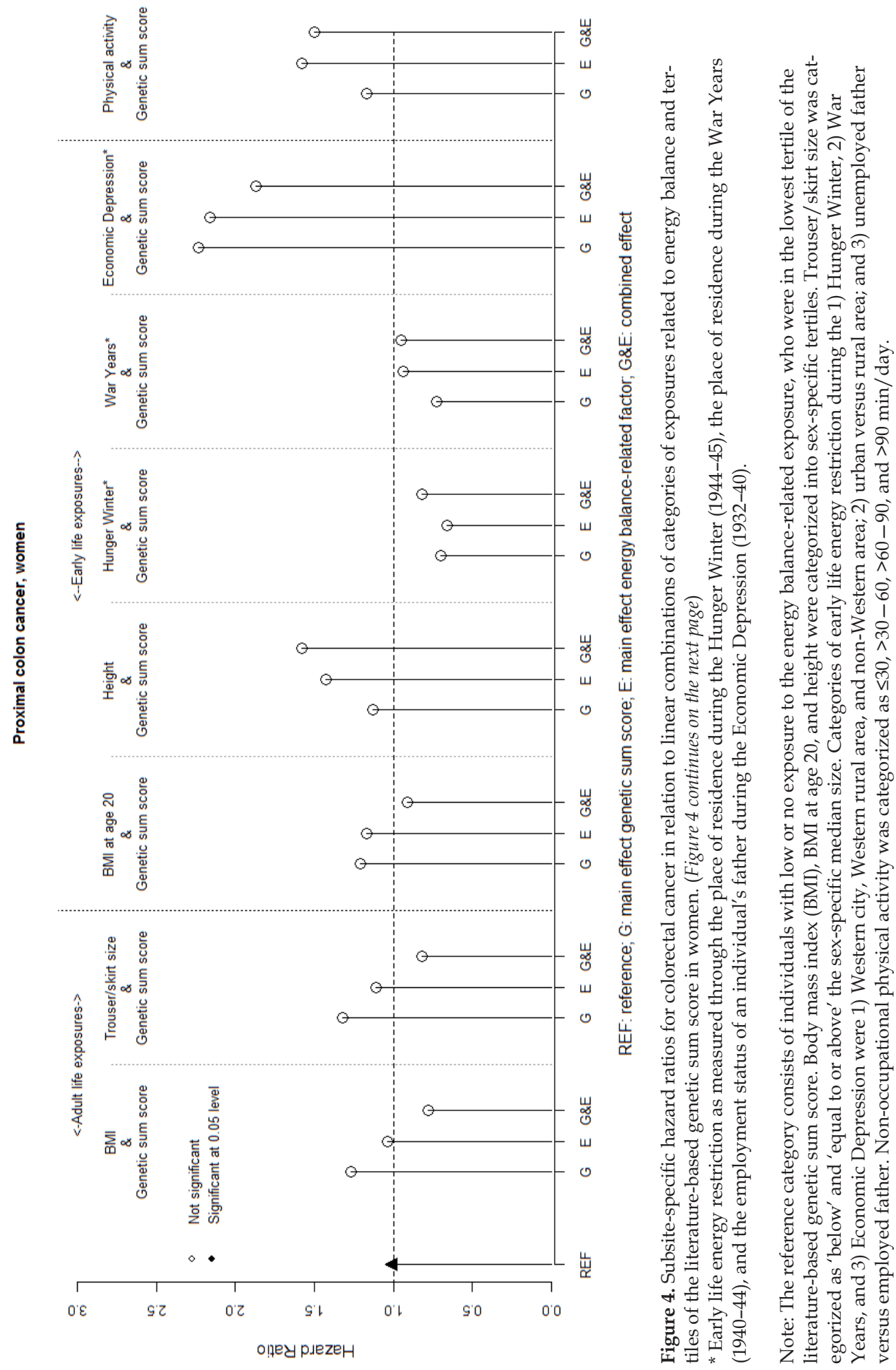

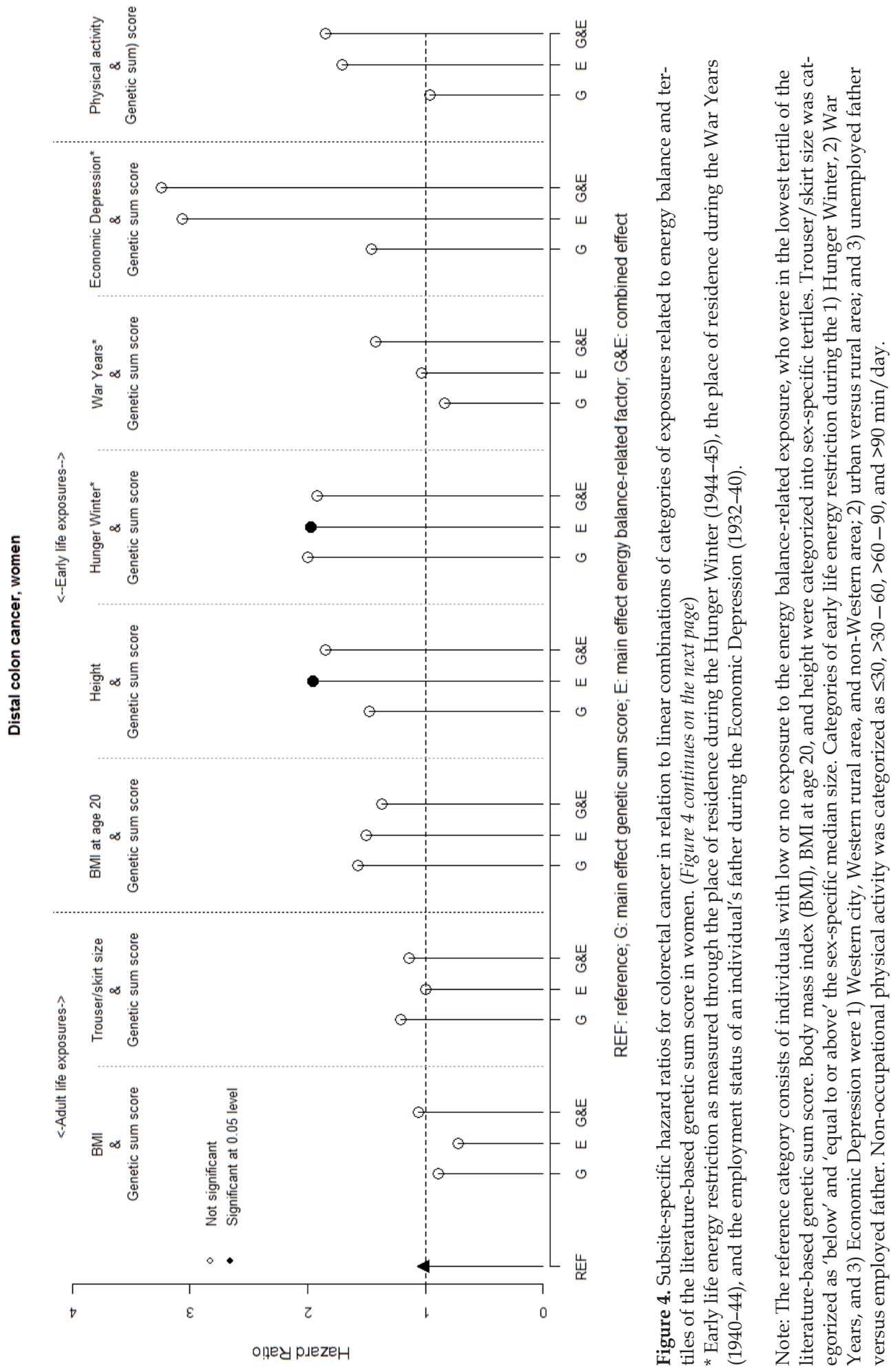

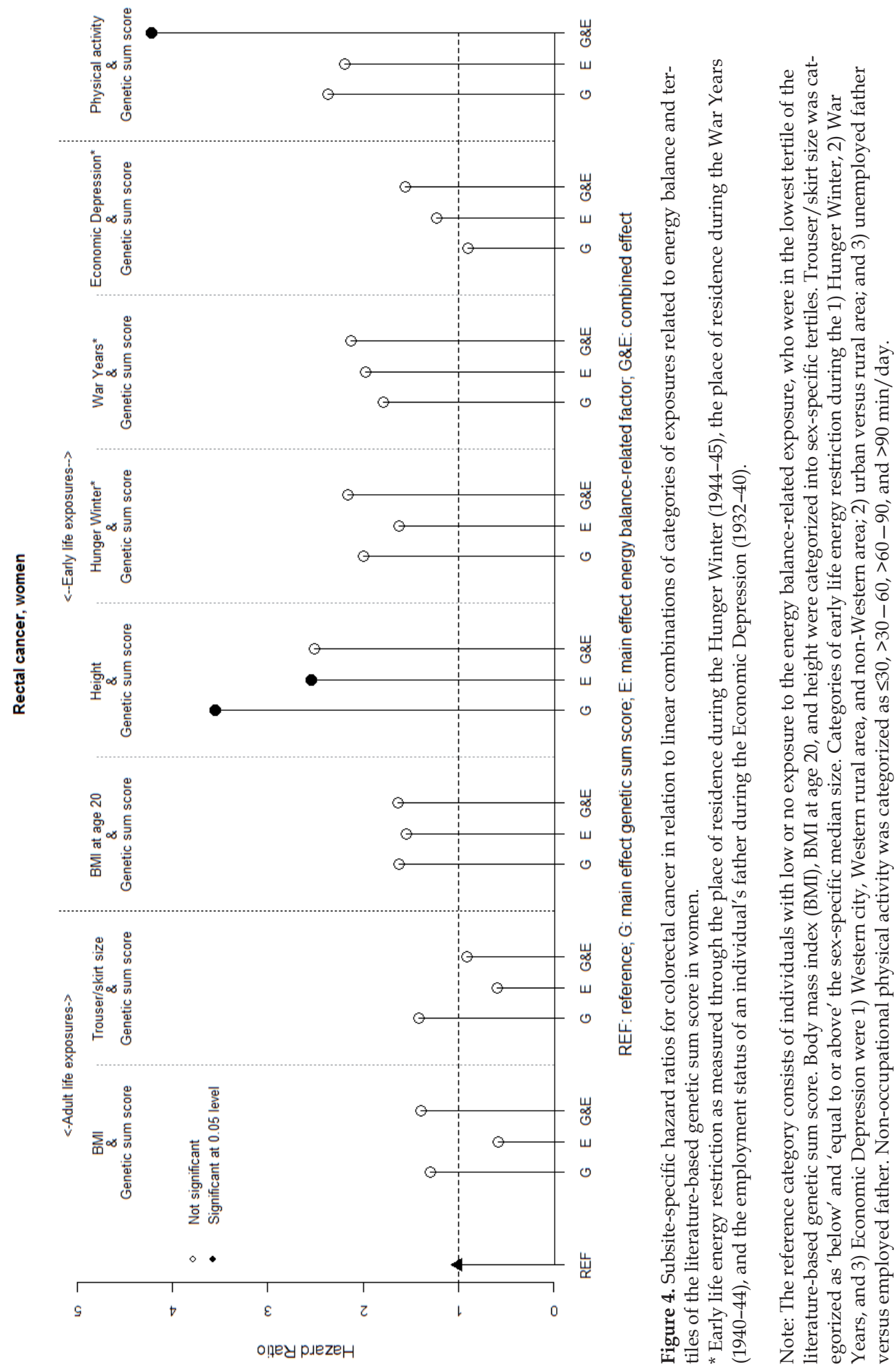
score which may have been reflected in this combined effect (Figure 3; Supplemental Table 2).

In women, a trend in favor of combined effects was less clear. Significantly increased risks were not confined to categories representing a high genetic sum score in combination with a larger body size, the absence of early life energy restriction or a low level of physical activity (Figure 4; Supplemental Table 3). The IGF1 19-CA repeat was associated with risk in women in analyses of combined effects, but this association was independent of energy balance-related factors. Significantly decreased risks were evident across colon subsites for non-19/non-19 carriers versus 19/19 carriers, and mirrored the strength of the marginal associations presented in Table 2 (therefore, data are not shown).

\subsection{Discussion}

To our knowledge, this is the most comprehensive study on potential combined effects of energy balance-related CRC risk factors and genetic variants in IGF pathway-related genes. Investigated in this study were adult life as well as early life exposures, including unique data on energy restriction during the Dutch Hunger Winter, War Years, and Economic Depression. Our study builds on previous publications showing indicators of energy balance to be CRC risk factors, ${ }^{4,5}$ and on publications reporting associations between genetic variants in IGF pathway-related genes and CRC risk. ${ }^{40,43,45,47,58-74}$ Only few studies have investigated combined effects between energy balance-related CRC risk factors and genetic variants in genes related to the IGF pathway and tested GxE interactions; the results in these studies were inconsistent. ${ }^{43,75,76}$

Our findings highlight the importance of the IGF pathway as a link between energy balance and incident colon cancer in men. Risks were between 1.9-2.5-fold in men with more central body fat, taller men and men without early life energy restriction, who were in the highest tertile of the literature-based genetic sum score, relative to those with more favorable profiles. Combined effects were evident for both proximal and distal colon cancer, while marginal associations based on NLCS data mainly showed associations with distal colon cancer. The absence of combined effects in women may be explained by that female reproductive hormones counteract insulin resistance. Experimental findings have shown that estrogens improved insulin sensitivity in obesity-induced mice, which influenced CRC progression. ${ }^{77}$ In addition, the European Prospective Investigation into Cancer observed a positive association between waist/hip ratio and colon cancer risk in postmenopausal women who did not use hormone replacement therapy (HRT), while this association was absent in those who did. ${ }^{78}$ Possibly, HRT counteracted effects of insulin resistance associated with abdominal fatness in these women. HRT in itself has been consistently associated with a reduced CRC risk. ${ }^{79}$

Findings with respect to the IGF1 CA repeat in this study indicated a strongly decreased proximal and distal colon cancer risk in female non-19/non-19 carriers versus 19/19 carriers, which was independent of energy balance. Results in previous studies were conflicting, showing an increased risk, $43,45,60$ decreased risk, ${ }^{73}$ and no association. ${ }^{40,47,61}$ It may be that the distribution of specific variant alleles in the non-19/non-19 group influences the direction of the association, and reconsideration of the commonly used IGF1 CA repeat categorization might be necessary in future studies.

Current data must be viewed in light of the BMI distribution in the NLCS, which was concentrated in the normal range at age 20 (18.5$<25 \mathrm{~kg} / \mathrm{m}^{2}$; categories defined by the World 
Health Organization ${ }^{80}$ ), and in the normal and overweight range in adulthood (18.5-<30 $\mathrm{kg} / \mathrm{m}^{2}$ ). A mere $0.4 \%$ of NLCS subcohort members was obese $\left(\geq 30 \mathrm{~kg} / \mathrm{m}^{2}\right)$ at age 20 , and $6.5 \%$ of subcohort members at adult age. As a result, more pronounced combined effects may be expected when comparing obese versus normal weight individuals, which would be possible in populations with higher obesity prevalences. On the gene side, the inclusion of more genetic variants from closely related pathways into the sum score could be rewarding, as pathways do not exist in isolation. A particular interesting pathway may be the mTOR pathway, which is downstream from the IGF pathway and which integrates signals from growth factors, nutrients, mutagens, and hormones in order to induce cell proliferation, resistance to apoptosis and autophagy. ${ }^{81}$ In women, the progesterone/estrogen pathway might be of interest for reasons explained above.

Alternatively, taking into account tumor heterogeneity seems of importance, because adult BMI and early life indicators of energy balance have been associated with the $\mathrm{CpG}$ island methylator phenotype (CIMP) in CRC and associated BRAF mutations in NLCS data. ${ }^{82-84}$ Major molecular alterations in colorectal tumors such as CIMP may be indicative of general disease pathways, culminating in tumors in specific colorectal sublocalizations. For example, CIMP has been associated with a proximal tumor location, whereas chromosomal instability has been associated with a distal location. ${ }^{85}$ The relative contribution of different disease pathways at the same time may render particular pathways more or less important, which could explain the absence of combined effects on rectal cancer risk at present.

The specific methodology used in this study requires discussion. First of all, our SNP selection strategy was aimed at minimizing the chance of selecting SNPs based on falsepositive results. To accomplish this, we included SNPs that were associated with CRC, diabetes, other obesity-related cancers and relevant traits (e.g. overweight and insulin resistance) in at least two different studies. We prioritized SNPs that were associated with several of these endpoints in order to select those SNPs that most likely interact with energy balance-related CRC risk factors. Selecting SNPs on the basis of potential main effects fitted with our mode of analysis (Cox regression), because main effects are inherently assumed in this technique when testing interactions. 7,8

Second, the use of a literature-based genetic sum score in which unfavorable alleles were aggregated demonstrated significant associations with proximal and distal colon cancer risk in men, and a borderline significant association with rectal cancer risk in women. Advantages of the use of a genetic sum score included a reduction in multiple testing and an optimization of power. In addition, no explicit assumptions had to be made regarding the inheritance models of the SNPs, as the misclassification associated with aggregating unfavorable alleles of SNPs was likely to even out under the assumption that one is not more likely to select SNPs that adhere to a dominant or recessive model. Several literature examples exist in which a genetic sum score has been shown successful for studying carcinogenic biomarkers, ${ }^{49,50,86}$ cancer risk, ${ }^{87-94}$ and cancer survival. ${ }^{91,95,96}$ However, in contrast to many of these studies, we based our choice of the unfavorable allele on previous studies instead of own data, to avoid overfitting of the cumulative model due to possible falsepositive findings in a single study population.

Despite the above considerations, multiplicative and additive interactions between the genetic sum score and energy balance-related exposures were generally not statistically sig- 
nificant in this study. As described in the introduction, power may have been an issue. It has also been described that statistical interaction does not equal biological interaction. Although biological interaction may best translate into a departure from additivity of risk differences, additivity of effects does not necessarily imply the absence of interaction. .53 Under the sufficient-component cause model, interaction may be defined as the participation of two component causes in the same sufficient cause. ${ }^{97}$ Therefore, that associations in men were repeatedly in the same direction, with significantly increased risks observed in men with the most adverse profiles, suggests the co-participation of energy balance and IGF pathway polymorphisms in CRC development in men, even though tests for interaction were not significant.

Limitations to our approach of modelling interactions may have included that we could not take into account the diploid character of the human genome, i.e. for those genes for which several SNPs were genotyped, the presence of multiple unfavorable alleles on the same parental chromosome might have resulted in a different gene product and associated disease risk than when alleles were distributed evenly across both chromosomes. It could also have been that a particular combination of the investigated SNPs interacted with energy balance-related factors and that the remaining SNPs introduced noise into the genetic sum score. Nevertheless, the use of a literature-based genetic sum score appears promising, considering that most single SNPdisease associations were not significant in this study.

In conclusion, specific energy balance-related CRC risk factors and SNPs in genes related to the IGF pathway jointly influenced colon cancer development in men. Associations may have been absent in women due to effects of estrogen-related factors, counteracting insulin resistance. The strength of this study is that combined effects were evident for multiple energy balance-related risk factors in the hypothesized direction. 


\section{References}

1. Iacopetta, B. Are there two sides to colorectal cancer? Int J Cancer 101, 403-408 (2002).

2. Li, F. \& Lai, M. Colorectal cancer, one entity or three. J Zhejiang Univ Sci B 10, 219-229 (2009).

3. Koo, J. H. \& Leong, R. W. L. Sex differences in epidemiological, clinical and pathological characteristics of colorectal cancer. J Gastroenterol Hepatol 25, 33-42 (2010).

4. World Cancer Research Fund / American Institute for Cancer Research. Food, Nutrition, Physical Activity, and the Prevention of Cancer: a Global Perspective. Washington DC: AICR, 2007.

5. Colorectal cancer | Continuous Update Project | WCRF. At: http:/ / www.wcrf.org. Accessed: Janu-ary 30, 2013.

6. Aschard, H. et al. Challenges and opportunities in genome-wide environmental interaction (GWEI) studies. Hum Genet 131, 1591-1613 (2012).

7. Greenland, S. Basic problems in interaction assessment. Environ Health Perspect 101 Suppl 4, 5966 (1993).

8. Khoury, M. J., Beaty, T. H. \& Cohen, B. H. Fundamentals of genetic epidemiology. New York, NY: Oxford University Press, 1993.

9. Kaaks, R. \& Lukanova, A. Energy balance and cancer: the role of insulin and insulin-like growth factor-I. Proc Nutr Soc 60, 91-106 (2001).

10. Yu, H. \& Rohan, T. Role of the insulin-like growth factor family in cancer development and progression. J Natl Cancer Inst 92, 1472-89 (2000).

11. Sandhu, M. S., Dunger, D. B. \& Giovannucci, E. L. Insulin, insulin-like growth factor-I (IGF-I), IGF binding proteins, their biologic interactions, and colorectal cancer. J Natl Cancer Inst 94, 972-80 (2002).

12. Durai, R., Yang, W., Gupta, S., Seifalian, A. M. \& Winslet, M. C. The role of the insulin-like growth factor system in colorectal cancer: review of current knowledge. Int J Colorectal Dis 20, 203-20 (2005).

13. Komninou, D., Ayonote, A., Richie, J. P. \& Rigas, B. Insulin resistance and its contribution to colon carcinogenesis. Exp Biol Med 228, 396-405 (2003).

14. Deng, L., Gui, Z., Zhao, L., Wang, J. \& Shen, L. Diabetes mellitus and the incidence of colorectal cancer: an updated systematic review and metaanalysis. Dig Dis Sci 57, 1576-1585 (2012)

15. Maher, B. Personal genomes: The case of the missing heritability. Nature 456, 18-21 (2008).

16. Van Steen, K. Travelling the world of gene-gene interactions. Brief Bioinformatics 13, 1-19 (2012).
17. Zhang, J. Genetic redundancies and their evolutionary maintenance. Adv Exp Med Biol 751, 279_ 300 (2012).

18. Van den Brandt, P. A. et al. A large-scale prospective cohort study on diet and cancer in The Netherlands. J Clin Epidemiol 43, 285-95 (1990).

19. Goldbohm, R. A. et al. Validation of a dietary questionnaire used in a large-scale prospective cohort study on diet and cancer. Eur J Clin Nutr 48, 253-65 (1994).

20. Goldbohm, R. A. et al. Reproducibility of a food frequency questionnaire and stability of dietary habits determined from five annually repeated measurements. Eur J Clin Nutr 49, 420-9 (1995).

21. Nevo Table. Dutch food composition table 19867. The Hague, Netherlands: Voorlichtingsbureau voor de Voeding, 1986.

22. Van Breda, S. G. et al. Toenails: an easily accessible and long-term stable source of DNA for genetic analyses in large-scale epidemiological studies. Clin Chem 53, 1168-70 (2007).

23. Goldbohm, R. A., van den Brandt, P. A. \& Dorant, E. Estimation of the coverage of Dutch municipalities by cancer registries and PALGA based on hospital discharge data. Tijdschr Soc Gezondheidsz 72, 80-84. (1994).

24. Casparie, M. et al. Pathology databanking and biobanking in The Netherlands, a central role for PALGA, the nationwide histopathology and cytopathology data network and archive. Cell Oncol 29, 19-24 (2007).

25. Hughes, L. A., Schouten, L. J., Goldbohm, R. A., van den Brandt, P. A. \& Weijenberg, M. P. Selfreported clothing size as a proxy measure for body size. Epidemiology 20, 673-6 (2009).

26. Moore, L. L. et al. BMI and waist circumference as predictors of lifetime colon cancer risk in Framingham Study adults. Int J Obes Relat Metab Disord 28, 559-67 (2004).

27. Wang, Y. et al. A prospective study of waist circumference and body mass index in relation to colorectal cancer incidence. Cancer Causes Control 19, 783-92 (2008).

28. Burger, G., Sandstead, H. \& Drummond, J. Malnutrition and Starvation in Western Netherlands, September 1944 to July 1945. Part I and II. The Hague, the Netherlands: General State Printing Office, 1948.

29. Trienekens, G. Tussen ons volk en de honger. De voedselvoorziening, 1940-45. (English translation: Between our nation and the hunger. The food supply, 1940-45). Utrecht, the Netherlands: Stichting Matrijs, 1985. 
30. Simons, C. C. et al. Physical activity, occupational sitting time, and colorectal cancer risk in the Netherlands cohort study. Am J Epidemiol 177, 514-530 (2013).

31. Hettinger, T. H., Mueller, B. H. \& Gebhard, H. Ermittlung des Arbeitsenergieumsatzes bei Dynamisch Muskulaerer Arbeit. Dortmund, Germany: Bundesarbeit fuer Arbeitsschutz; 1989.

32. Cline, R. E., Laurent, N. M. \& Foran, D. R. The fingernails of Mary Sullivan: developing reliable methods for selectively isolating endogenous and exogenous DNA from evidence. J Forensic Sci 48, 328-333 (2003).

33. Oeth, P. et al. iPLEXTM Assay: Increased Plexing Efficiency and Flexibility for MassARRAY® System Through Single Base Primer Extension with Mass-Modified Terminators. Freely available online.

34. Ali, O., Cohen, P. \& Lee, K. W. Epidemiology and biology of insulin-like growth factor binding protein-3 (IGFBP-3) as an anti-cancer molecule. Horm Metab Res 35, 726-33 (2003).

35. Domené, H. M., Hwa, V., Jasper, H. G. \& Rosenfeld, R. G. Acid-labile subunit (ALS) deficiency. Best Pract Res Clin Endocrinol Metab 25, 101-113 (2011).

36. Yee, D. Insulin-like Growth Factor Receptor Inhibitors: Baby or the Bathwater? J Natl Cancer Inst 104, 975-981 (2012).

37. Dearth, R., Cui, X., Kim, H., Hadsell, D. \& Lee, A. Oncogenic transformation by the signaling adaptor proteins insulin receptor substrate (IRS)-1 and IRS-2. Cell Cycle 15, 705-13

38. Bull, A. The role of peroxisome proliferatoractivated receptor gamma in colon cancer and inflammatory bowel disease. Arch Pathol Lab Med 127, 1121-3

39. Pais, R., Silaghi, H., Silaghi, A. C., Rusu, M. L. \& Dumitrascu, D. L. Metabolic syndrome and risk of subsequent colorectal cancer. World J Gastroenterol. 15, 5141-5148 (2009).

40. Slattery, M. L. et al. Associations among IRS1, IRS2, IGF1, and IGFBP3 genetic polymorphisms and colorectal cancer. Cancer Epidemiol Biomarkers Prev 13, 1206-14 (2004).

41. Neuhausen, S. L. et al. Prostate cancer risk and IRS1, IRS2, IGF1, and INS polymorphisms: strong association of IRS1 G972R variant and cancer risk. Prostate 64, 168-174 (2005).

42. Schildkraut, J. M. et al. IGF1 (CA)19 repeat and IGFBP3 -202 A/C genotypes and the risk of prostate cancer in Black and White men. Cancer Epidemiol Biomarkers Prev 14, 403-408 (2005).
43. Slattery, M. L. et al. Energy balance, insulinrelated genes and risk of colon and rectal cancer. Int J Cancer 115, 148-54 (2005).

44. Sweeney, C. et al. Insulin-like growth factor pathway polymorphisms associated with body size in Hispanic and non-Hispanic white women. Cancer Epidemiol Biomarkers Prev 14, 1802-1809 (2005).

45. Samowitz, W. S. et al. Polymorphisms in insulinrelated genes predispose to specific KRAS2 and TP53 mutations in colon cancer. Mutat Res 595, 117-24 (2006).

46. Slattery, M. L. et al. PPARgamma and colon and rectal cancer: associations with specific tumor mutations, aspirin, ibuprofen and insulin-related genes (United States). Cancer Causes Control 17, 239-49 (2006).

47. Pechlivanis, S. et al. Polymorphisms in the insulin like growth factor 1 and IGF binding protein 3 genes and risk of colorectal cancer. Cancer Detect Prev 31, 408-16 (2007).

48. Huang, Q., Wang, C., Qiu, L. J., Shao, F. \& Yu, J. $\mathrm{H}$. The association between IGF1 CA repeat polymorphisms and breast cancer risk: a metaanalysis. Breast Cancer Res Treat 129, 191-194 (2011).

49. Ketelslegers, H. B. et al. Interindividual variations in DNA adduct levels assessed by analysis of multiple genetic polymorphisms in smokers. Cancer Epidemiol Biomarkers Prev 15, 624-629 (2006).

50. Langie, S. A. et al. Modulation of nucleotide excision repair in human lymphocytes by genetic and dietary factors. Br J Nutr 103, 490-501 (2010).

51. Barlow, W. E. Robust variance estimation for the case-cohort design. Biometrics 50, 1064-1072 (1994).

52. Schoenfeld, D. Partial residuals for the proportional hazards regression model. Biometrika 69, 239-241 (1982).

53. Rothman, K. J. \& Greenland, S. Modern Epidemiology. Philadelphia, PA: Lippincott-Raven Publishers, 1998.

54. Carpenter, J. \& Bithell, J. Bootstrap confidence intervals: when, which, what? A practical guide for medical statisticians. Stat Med 19, 1141-1164 (2000).

55. Knol, M. J. et al. Estimating measures of interaction on an additive scale for preventive exposures. Eur J Epidemiol 26, 433-438 (2011).

56. Hughes, L. A. et al. Body size and colorectal cancer risk after 16.3 years of follow-up: an analysis from the Netherlands Cohort Study. Am J Epidemiol 174, 1127-1139 (2011). 
57. Hughes, L. A. et al. Childhood and adolescent energy restriction and subsequent colorectal cancer risk: results from the Netherlands Cohort Study. Int J Epidemiol 39, 1333-1344 (2010).

58. Landi, S. et al. Association of common polymorphisms in inflammatory genes interleukin (IL)6, IL8, tumor necrosis factor alpha, NFKB1, and peroxisome proliferator-activated receptor gamma with colorectal cancer. Cancer Res 63, 3560-6 (2003).

59. Jiang, J. et al. Influence of the C161T but not Pro12Ala polymorphism in the peroxisome proliferator-activated receptor-gamma on colorectal cancer in an Indian population. Cancer Sci 96, 507-12 (2005).

60. Morimoto, L. M., Newcomb, P. A., White, E., Bigler, J. \& Potter, J. D. Insulin-like growth factor polymorphisms and colorectal cancer risk. Cancer Epidemiol Biomarkers Prev 14, 1204-11 (2005).

61. Wong, H. L. et al. A new single nucleotide polymorphism in the insulin-like growth factor I regulatory region associates with colorectal cancer risk in singapore chinese. Cancer Epidemiol Biomarkers Prev 14, 144-51 (2005).

62. Koh, W. P., Yuan, J. M., Van Den Berg, D., Ingles, S. A. \& Yu, M. C. Peroxisome proliferatoractivated receptor (PPAR) gamma gene polymorphisms and colorectal cancer risk among Chinese in Singapore. Carcinogenesis 27, 1797-802 (2006).

63. Theodoropoulos, G. et al. Relation between common polymorphisms in genes related to inflammatory response and colorectal cancer. World J Gastroenterol 12, 5037-43 (2006).

64. Kaklamani, V. G. et al. Variants of the adiponectin (ADIPOQ) and adiponectin receptor 1 (ADIPOR1) genes and colorectal cancer risk. JAMA 300, 1523-31 (2008).

65. Kury, S. et al. Low-penetrance alleles predisposing to sporadic colorectal cancers: a French casecontrolled genetic association study. BMC Cancer 8, 326 (2008).

66. Wong, H. L. et al. Insulin-like growth factor-1 promoter polymorphisms and colorectal cancer: a functional genomics approach. Gut 57, 1090-6 (2008).

67. Feik, E. et al. Association of IGF1 and IGFBP3 polymorphisms with colorectal polyps and colorectal cancer risk. Cancer Causes Control 21, 91-7 (2009).

68. Tsilidis, K. K. et al. Association of common polymorphisms in IL10, and in other genes related to inflammatory response and obesity with colorectal cancer. Cancer Causes Control 20, 1739-51 (2009).
69. Xiang, H. et al. Association between two functional polymorphisms of insulin-like growth factor binding protein 3 and colorectal cancer risk in a Chinese population. J Toxicol Environ Health 72, 706-11 (2009).

70. Gao, C. M. et al. Relationship between growth hormone 1 genetic polymorphism and susceptibility to colorectal cancer. J Hum Genet 55, 163-6 (2010).

71. Xu, W. et al. PPARgamma polymorphisms and cancer risk: a meta-analysis involving 32,138 subjects. Oncol Rep 24, 579-85 (2010).

72. He, B. et al. Effects of genetic variations in the adiponectin pathway genes on the risk of colorectal cancer in the Chinese population. BMC Med Genet 12, 94 (2011).

73. Keku, T. O. et al. Genetic variants in IGF-I, IGF-II, IGFBP-3, and adiponectin genes and colon cancer risk in African Americans and Whites. Cancer Causes Control 23, 1127-38 (2012).

74. Slattery, M. L. et al. Colon tumor mutations and epigenetic changes associated with genetic polymorphism: insight into disease pathways. Mutat Res 660, 12-21 (2009).

75. Khoury-Shakour, S. et al. Recreational physical activity modifies the association between a common GH1 polymorphism and colorectal cancer risk. Cancer Epidemiol Biomarkers Prev 17, 3314-8 (2008).

76. Liu, L. et al. Interactions between genetic variants in the adiponectin, adiponectin receptor 1 and environmental factors on the risk of colorectal cancer. PLoS One 6, e27301 (2011).

77. Rondini, E. A., Harvey, A. E., Steibel, J. P., Hursting, S. D. \& Fenton, J. I. Energy balance modulates colon tumor growth: Interactive roles of insulin and estrogen. Mol Carcinog 50, 370-382 (2011).

78. Pischon, T. et al. Body size and risk of colon and rectal cancer in the European Prospective Investigation Into Cancer and Nutrition (EPIC). J Natl Cancer Inst 98, 920-931 (2006).

79. Grodstein, F., Newcomb, P. A. \& Stampfer, M. J. Postmenopausal hormone therapy and the risk of colorectal cancer: a review and meta-analysis. Am J Med 106, 574-582 (1999).

80. WHO: Global Database on Body Mass Index. At: http://apps.who.int/bmi/index.jsp?introPage=i ntro_3.html. Accessed: January 30, 2013.

81. Weijenberg, M. P. et al. The mTOR pathway and the role of energy balance throughout life in colorectal cancer etiology and prognosis: unravelling mechanisms through a multidimensional molecular epidemiologic approach. Curr Nutr Rep 2, 19 26 (2013). 
82. Hughes, L. A. et al. Early life exposure to famine and colorectal cancer risk: a role for epigenetic mechanisms. PLoS One 4, e7951 (2009).

83. Hughes, L. A. et al. Body size, physical activity and risk of colorectal cancer with or without the CpG island methylator phenotype (CIMP). PLoS One 6, e18571 (2011).

84. Hughes, L. A. et al. Body size and risk for colorectal cancers showing BRAF mutations or microsatellite instability: a pooled analysis. Int J Epidemiol 41, 1060-1072 (2012)

85. Issa, J. P. Colon cancer: it's CIN or CIMP. Clin Cancer Res 14, 5939-40 (2008).

86. Peluso, M. et al. Smoking, DNA adducts and number of risk DNA repair alleles in lung cancer cases, in subjects with benign lung diseases and in controls. J Nucleic Acids 2010, 386798 (2010).

87. Wang, W. et al. Genetic variants in cell cycle control pathway confer susceptibility to lung cancer. Clin Cancer Res 13, 5974-5981 (2007).

88. Yang, H. et al. Profiling of genetic variations in inflammation pathway genes in relation to bladder cancer predisposition. Clin Cancer Res 14, 2236-2244 (2008).

89. Ye, Y. et al. Genetic variations in microRNArelated genes are novel susceptibility loci for esophageal cancer risk. Cancer Prev Res 1, 460-469 (2008).

90. Bao, B. Y. et al. Individual and cumulative association of prostate cancer susceptibility variants with clinicopathologic characteristics of the disease. Clin Chim Acta 411, 1232-1237 (2010).

91. Liang, D. et al. Genetic variants in MicroRNA biosynthesis pathways and binding sites modify ovarian cancer risk, survival, and treatment response. Cancer Res 70, 9765-9776 (2010).

92. Yin, J. et al. Genetic variants in TGF- $\beta$ pathway are associated with ovarian cancer risk. PLoS One 6, e25559 (2011).

93. Chang, J., Dinney, C. P., Huang, M., Wu, X. \& Gu, $\mathrm{J}$. Genetic variants in telomere-maintenance genes and bladder cancer risk. PLoS One 7, e30665 (2012).

94. Slattery, M. L., Lundgreen, A., Wolff, R. K., Herrick, J. S. \& Caan, B. J. Genetic variation in the transforming growth factor- $\beta$-signaling pathway, lifestyle factors, and risk of colon or rectal cancer. Dis Colon Rectum 55, 532-540 (2012).

95. Lin, M. et al. Genetic variations in the transforming growth factor-beta pathway as predictors of survival in advanced non-small cell lung cancer. Carcinogenesis 32, 1050-1056 (2011).

96. Slattery, M. L., Lundgreen, A., Herrick, J. S., Wolff, R. K. \& Caan, B. J. Genetic variation in the transforming growth factor- $\beta$ signaling pathway and survival after diagnosis with colon and rectal cancer. Cancer 117, 4175-4183 (2011).

97. Rothman, K. J. \& Greenland, S. Causation and causal inference in epidemiology. Am J Public Health 95 Suppl 1, S144-150 (2005). 
Supplemental Table 1. Age-adjusted hazard ratios (HR) and 95\% confidence intervals (CI) for colorectal cancer in relation to SNPs in genes related to the IGF pathway in men and women in the Netherlands Cohort Study (1986-2002)

\begin{tabular}{|c|c|c|c|c|c|c|c|c|}
\hline & \multicolumn{8}{|c|}{ Colorectal cancer } \\
\hline & \multicolumn{4}{|c|}{ Men } & \multicolumn{4}{|c|}{ Women } \\
\hline & PY & $\mathrm{N}$ cases & HR & $(95 \% \mathrm{CI})$ & PY & $\mathrm{N}$ cases & HR & $(95 \% \mathrm{CI})$ \\
\hline \multicolumn{9}{|l|}{ IGF1 } \\
\hline \multicolumn{9}{|l|}{ rs1520220 } \\
\hline $\mathrm{CC}$ & 15,286 & 895 & 1 & reference & 15,700 & 603 & 1 & reference \\
\hline CG & 6,178 & 414 & 1.15 & $(0.97,1.35)$ & 7,207 & 277 & 1.00 & $(0.83,1.19)$ \\
\hline GG & 704 & 54 & 1.37 & $(0.92,2.05)$ & 765 & 31 & 1.03 & $(0.65,1.63)$ \\
\hline$P$-trend & & & & 0.04 & & & & 0.98 \\
\hline \multicolumn{9}{|l|}{ rs5742678 } \\
\hline $\mathrm{CC}$ & 12,877 & 745 & 1 & reference & 13,025 & 497 & 1 & reference \\
\hline GC & 7,912 & 519 & 1.14 & $(0.98,1.34)$ & 9,086 & 355 & 1.01 & $(0.85,1.20)$ \\
\hline GG & 1,379 & 98 & 1.25 & $(0.92,1.69)$ & 1,561 & 59 & 0.98 & $(0.69,1.37)$ \\
\hline$P$-trend & & & & 0.05 & & & & 0.97 \\
\hline \multicolumn{9}{|l|}{ rs5742694 } \\
\hline TT & 13,469 & 778 & 1 & reference & 13,695 & 518 & 1 & reference \\
\hline GT & 7,462 & 487 & 1.13 & $(0.97,1.33)$ & 8,568 & 341 & 1.03 & $(0.86,1.22)$ \\
\hline GG & 1,237 & 96 & 1.38 & $(1.01,1.88)$ & 1,410 & 51 & 0.96 & $(0.67,1.37)$ \\
\hline$P$-trend & & & & 0.02 & & & & 0.95 \\
\hline \multicolumn{9}{|l|}{ rs10735380 } \\
\hline AA & 12,365 & 724 & 1 & reference & 12,983 & 469 & 1 & reference \\
\hline AG & 8,346 & 533 & 1.10 & $(0.94,1.28)$ & 9,052 & 378 & 1.14 & $(0.96,1.35)$ \\
\hline GG & 1,456 & 105 & 1.25 & $(0.93,1.67)$ & 1,638 & 64 & 1.06 & $(0.76,1.47)$ \\
\hline$P$-trend & & & & 0.09 & & & & 0.26 \\
\hline \multicolumn{9}{|l|}{ IGFBP3 } \\
\hline \multicolumn{9}{|l|}{ rs2854744 } \\
\hline AA & 4,654 & 279 & 1 & reference & 4,778 & 193 & 1 & reference \\
\hline CA & 11,173 & 690 & 1.04 & $(0.85,1.26)$ & 11,750 & 446 & 0.93 & $(0.75,1.15)$ \\
\hline $\mathrm{CC}$ & 6,341 & 393 & 1.03 & $(0.83,1.28)$ & 7,128 & 270 & 0.96 & $(0.76,1.22)$ \\
\hline$P$-trend & & & & 0.80 & & & & 0.82 \\
\hline \multicolumn{9}{|l|}{ rs2132572 } \\
\hline GG & 13,360 & 768 & 1 & reference & 13,901 & 518 & 1 & reference \\
\hline GA & 7,661 & 510 & 1.12 & $(0.96,1.31)$ & 8,476 & 343 & 1.07 & $(0.90,1.27)$ \\
\hline AA & 1,147 & 85 & 1.29 & $(0.93,1.79)$ & 1,295 & 50 & 1.06 & $(0.73,1.54)$ \\
\hline$P$-trend & & & & 0.06 & & & & 0.49 \\
\hline \multicolumn{9}{|l|}{ rs35440925 } \\
\hline $\mathrm{CC}$ & 13,704 & 882 & 1 & reference & 14,615 & 579 & 1 & reference \\
\hline CG & 7,479 & 422 & 0.89 & $(0.76,1.05)$ & 8,113 & 294 & 0.92 & $(0.77,1.10)$ \\
\hline GG & 985 & 59 & 0.97 & $(0.67,1.39)$ & 944 & 37 & 1.02 & $(0.67,1.57)$ \\
\hline$P$-trend & & & & 0.27 & & & & 0.54 \\
\hline
\end{tabular}

Continues on the next page 


\begin{tabular}{|c|c|c|c|c|c|c|c|c|}
\hline & \multicolumn{8}{|c|}{ Colorectal cancer } \\
\hline & \multicolumn{4}{|c|}{ Men } & \multicolumn{4}{|c|}{ Women } \\
\hline & PY & $\mathrm{N}$ cases & HR & $(95 \% \mathrm{CI})$ & PY & $\mathrm{N}$ cases & HR & $(95 \% \mathrm{CI})$ \\
\hline \multicolumn{9}{|l|}{ IRS1 } \\
\hline \multicolumn{9}{|l|}{ rs1801278 } \\
\hline GG & 19,004 & 1,155 & 1 & reference & 20,576 & 789 & 1 & reference \\
\hline GA & 3,017 & 203 & 1.11 & $(0.90,1.37)$ & 3,047 & 119 & 1.03 & $(0.80,1.31)$ \\
\hline AA & 147 & 5 & 0.60 & $(0.20,1.77)$ & 49 & 3 & 2.33 & $(0.47,11.65)$ \\
\hline$P$-trend & & & & 0.58 & & & & 0.66 \\
\hline \multicolumn{9}{|l|}{ IRS2 } \\
\hline \multicolumn{9}{|l|}{ rs1805097 } \\
\hline $\mathrm{AA}$ & 2,709 & 151 & 1 & reference & 3,018 & 111 & 1 & reference \\
\hline GA & 10,044 & 604 & 1.07 & $(0.84,1.36)$ & 10,621 & 405 & 1.01 & $(0.77,1.32)$ \\
\hline GG & 9,274 & 603 & 1.15 & $(0.90,1.47)$ & 9,985 & 392 & 1.07 & $(0.82,1.40)$ \\
\hline$P$-trend & & & & 0.20 & & & & 0.51 \\
\hline \multicolumn{9}{|l|}{ rs2289046 } \\
\hline GG & 2,511 & 134 & 1 & reference & 2,838 & 109 & 1 & reference \\
\hline GA & 10,059 & 607 & 1.13 & $(0.88,1.46)$ & 10,676 & 398 & 0.94 & $(0.72,1.23)$ \\
\hline AA & 9,598 & 622 & 1.20 & $(0.93,1.55)$ & 10,158 & 404 & 1.03 & $(0.78,1.34)$ \\
\hline$P$-trend & & & & 0.15 & & & & 0.57 \\
\hline \multicolumn{9}{|l|}{ rs754204 } \\
\hline $\mathrm{CC}$ & 5,876 & 336 & 1 & reference & 6,496 & 248 & 1 & reference \\
\hline $\mathrm{TC}$ & 11,128 & 694 & 1.08 & $(0.91,1.30)$ & 11,468 & 438 & 0.98 & $(0.81,1.20)$ \\
\hline TT & 4,754 & 326 & 1.17 & $(0.95,1.45)$ & 5,291 & 215 & 1.04 & $(0.82,1.31)$ \\
\hline$P$-trend & & & & 0.14 & & & & 0.78 \\
\hline \multicolumn{9}{|l|}{ rs4773082 } \\
\hline TT & 6,560 & 357 & 1 & reference & 6,954 & 249 & 1 & reference \\
\hline CT & 10,190 & 623 & 1.11 & $(0.93,1.33)$ & 10,299 & 382 & 1.00 & $(0.82,1.23)$ \\
\hline $\mathrm{CC}$ & 3,813 & 243 & 1.12 & $(0.89,1.41)$ & 4,082 & 152 & 1.03 & $(0.80,1.33)$ \\
\hline$P$-trend & & & & 0.27 & & & & 0.81 \\
\hline \multicolumn{9}{|l|}{ GHRHR } \\
\hline \multicolumn{9}{|l|}{ rs4988496 } \\
\hline GG & 19,743 & 1,204 & 1 & reference & 20,791 & 813 & 1 & reference \\
\hline AG & 2,346 & 153 & 1.05 & $(0.83,1.33)$ & 2,783 & 93 & 0.86 & $(0.66,1.12)$ \\
\hline AA & 63 & 4 & 1.02 & $(0.25,4.10)$ & 98 & 5 & 1.32 & $(0.40,4.38)$ \\
\hline$P$-trend & & & & 0.72 & & & & 0.39 \\
\hline \multicolumn{9}{|l|}{ PPARG } \\
\hline \multicolumn{9}{|l|}{ rs1801282 } \\
\hline GG & 187 & 12 & 1 & reference & 389 & 12 & 1 & reference \\
\hline GC & 4,814 & 280 & 0.86 & $(0.38,1.95)$ & 4,916 & 194 & 1.26 & $(0.61,2.58)$ \\
\hline $\mathrm{CC}$ & 17,151 & 1,071 & 0.92 & $(0.41,2.06)$ & 18,363 & 705 & 1.21 & $(0.60,2.44)$ \\
\hline$P$-trend & & & & 0.58 & & & & 0.93 \\
\hline
\end{tabular}

Continues on the next page 


\begin{tabular}{|c|c|c|c|c|c|c|c|c|}
\hline & \multicolumn{8}{|c|}{ Colorectal cancer } \\
\hline & \multicolumn{4}{|c|}{ Men } & \multicolumn{4}{|c|}{ Women } \\
\hline & PY & $\mathrm{N}$ cases & HR & $(95 \% \mathrm{CI})$ & PY & $\mathrm{N}$ cases & HR & $(95 \% \mathrm{CI})$ \\
\hline \multicolumn{9}{|l|}{$A D I P O Q$} \\
\hline \multicolumn{9}{|l|}{ rs1501299 } \\
\hline AA & 1,620 & 103 & 1 & reference & 1,710 & 51 & 1 & reference \\
\hline CA & 8,834 & 108 & 0.92 & $(0.68,1.23)$ & 8,779 & 366 & 1.39 & $(0.97,1.98)$ \\
\hline $\mathrm{CC}$ & 11,715 & 751 & 1.03 & $(0.77,1.37)$ & 13,184 & 493 & 1.26 & $(0.89,1.79)$ \\
\hline$P$-trend & & & & 0.34 & & & & 0.88 \\
\hline \multicolumn{9}{|l|}{ rs2241766 } \\
\hline TT & 17,305 & 1,103 & 1 & reference & 18,368 & 729 & 1 & reference \\
\hline GT & 4,562 & 244 & 0.83 & $(0.69,1.00)$ & 4,849 & 164 & 0.85 & $(0.68,1.05)$ \\
\hline GG & 170 & 10 & 0.91 & $(0.38,2.16)$ & 358 & 16 & 1.10 & $(0.57,2.12)$ \\
\hline$P$-trend & & & & 0.06 & & & & 0.25 \\
\hline \multicolumn{9}{|l|}{ rs266729 } \\
\hline GG & 1,329 & 93 & 1 & reference & 1,909 & 60 & 1 & reference \\
\hline GC & 8,742 & 545 & 0.90 & $(0.66,1.23)$ & 8,479 & 334 & 1.32 & $(0.94,1.86)$ \\
\hline $\mathrm{CC}$ & 12,098 & 724 & 0.84 & $(0.62,1.15)$ & 13,285 & 517 & 1.29 & $(0.93,1.80)$ \\
\hline$P$-trend & & & & 0.22 & & & & 0.36 \\
\hline \multicolumn{9}{|l|}{ rs1648707 } \\
\hline $\mathrm{AA}$ & 9,974 & 597 & 1 & reference & 10,948 & 426 & 1 & reference \\
\hline CA & 10,041 & 616 & 1.03 & $(0.88,1.21)$ & 9,970 & 393 & 1.00 & $(0.84,1.19)$ \\
\hline $\mathrm{CC}$ & 2,153 & 150 & 1.19 & $(0.92,1.53)$ & 2,754 & 92 & 0.81 & $(0.62,1.08)$ \\
\hline$P$-trend & & & & 0.25 & & & & 0.27 \\
\hline \multicolumn{9}{|l|}{ rs182052 } \\
\hline GG & 9,900 & 592 & 1 & reference & 10,891 & 424 & 1 & reference \\
\hline GA & 10,085 & 617 & 1.03 & $(0.88,1.21)$ & 9,966 & 393 & 1.00 & $(0.84,1.19)$ \\
\hline AA & 2,183 & 154 & 1.20 & $(0.93,1.54)$ & 2,816 & 94 & 0.82 & $(0.62,1.08)$ \\
\hline$P$-trend & & & & 0.23 & & & & 0.29 \\
\hline \multicolumn{9}{|l|}{ ADIPOR1 } \\
\hline \multicolumn{9}{|l|}{ rs1342387 } \\
\hline $\mathrm{AA}$ & 4,266 & 234 & 1 & reference & 4,555 & 195 & 1 & reference \\
\hline GA & 11,418 & 725 & 1.16 & $(0.95,1.42)$ & 12,350 & 447 & 0.86 & $(0.69,1.06)$ \\
\hline GG & 6,484 & 403 & 1.12 & $(0.90,1.40)$ & 6,751 & 267 & 0.94 & $(0.74,1.20)$ \\
\hline$P$-trend & & & & 0.42 & & & & 0.77 \\
\hline \multicolumn{9}{|l|}{ rs7539542 } \\
\hline $\mathrm{CC}$ & 10,697 & 671 & 1 & reference & 11,152 & 469 & 1 & reference \\
\hline CG & 9,496 & 566 & 0.95 & $(0.82,1.11)$ & 10,282 & 361 & 0.84 & $(0.71,1.00)$ \\
\hline GG & 1,957 & 125 & 0.99 & $(0.76,1.30)$ & 2,130 & 81 & 0.95 & $(0.70,1.29)$ \\
\hline$P$-trend & & & & 0.72 & & & & 0.19 \\
\hline \multicolumn{9}{|l|}{ rs12733285 } \\
\hline $\mathrm{CC}$ & 1,884 & 94 & 1 & reference & 1,610 & 80 & 1 & reference \\
\hline $\mathrm{CT}$ & 9,301 & 573 & 1.27 & $(0.95,1.70)$ & 9,981 & 370 & 0.76 & $(0.56,1.05)$ \\
\hline $\mathrm{TT}$ & 10,971 & 696 & 1.29 & $(0.97,1.72)$ & 12,008 & 459 & 0.80 & $(0.58,1.09)$ \\
\hline$P$-trend & & & & 0.19 & & & & 0.51 \\
\hline
\end{tabular}




\begin{tabular}{|c|c|c|c|c|c|c|c|c|}
\hline & \multicolumn{8}{|c|}{ Colorectal cancer } \\
\hline & \multicolumn{4}{|c|}{ Men } & \multicolumn{4}{|c|}{ Women } \\
\hline & PY & $\mathrm{N}$ cases & $\mathrm{HR}$ & $(95 \% \mathrm{CI})$ & PY & $\mathrm{N}$ cases & HR & $(95 \%$ CI $)$ \\
\hline \multicolumn{9}{|l|}{ ADIPOR2 } \\
\hline \multicolumn{9}{|l|}{ rs1044471 } \\
\hline $\mathrm{CC}$ & 6,286 & 409 & 1 & reference & 6,861 & 240 & 1 & reference \\
\hline CT & 11,233 & 660 & 0.90 & $(0.76,1.07)$ & 11,637 & 473 & 1.12 & $(0.92,1.36)$ \\
\hline TT & 4,614 & 293 & 0.98 & $(0.79,1.21)$ & 5,141 & 197 & 1.08 & $(0.85,1.37)$ \\
\hline$P$-trend & & & & 0.74 & & & & 0.47 \\
\hline \multicolumn{9}{|l|}{ rs767870 } \\
\hline TT & 14,593 & 923 & 1 & reference & 16,325 & 609 & 1 & reference \\
\hline $\mathrm{TC}$ & 6,917 & 403 & 0.91 & $(0.77,1.07)$ & 6,664 & 278 & 1.13 & $(0.94,1.35)$ \\
\hline $\mathrm{CC}$ & 659 & 37 & 0.87 & $(0.56,1.36)$ & 668 & 23 & 0.93 & $(0.56,1.57)$ \\
\hline$P$-trend & & & & 0.22 & & & & 0.36 \\
\hline
\end{tabular}

Abbreviations: ADIPOQ, adiponectin; ADIPOR1 and -2, adiponectin receptor 1 and 2; GHRHR, growth hormone releasing hormone receptor; IGF1, insulin-like growth factor 1; IGFBP3, insulin-like growth factor binding protein 3; IRS1 and -2, insulin receptor substrate 1 and 2; PPARG, peroxisome proliferator-activated receptor gamma; PY, person-years at risk 


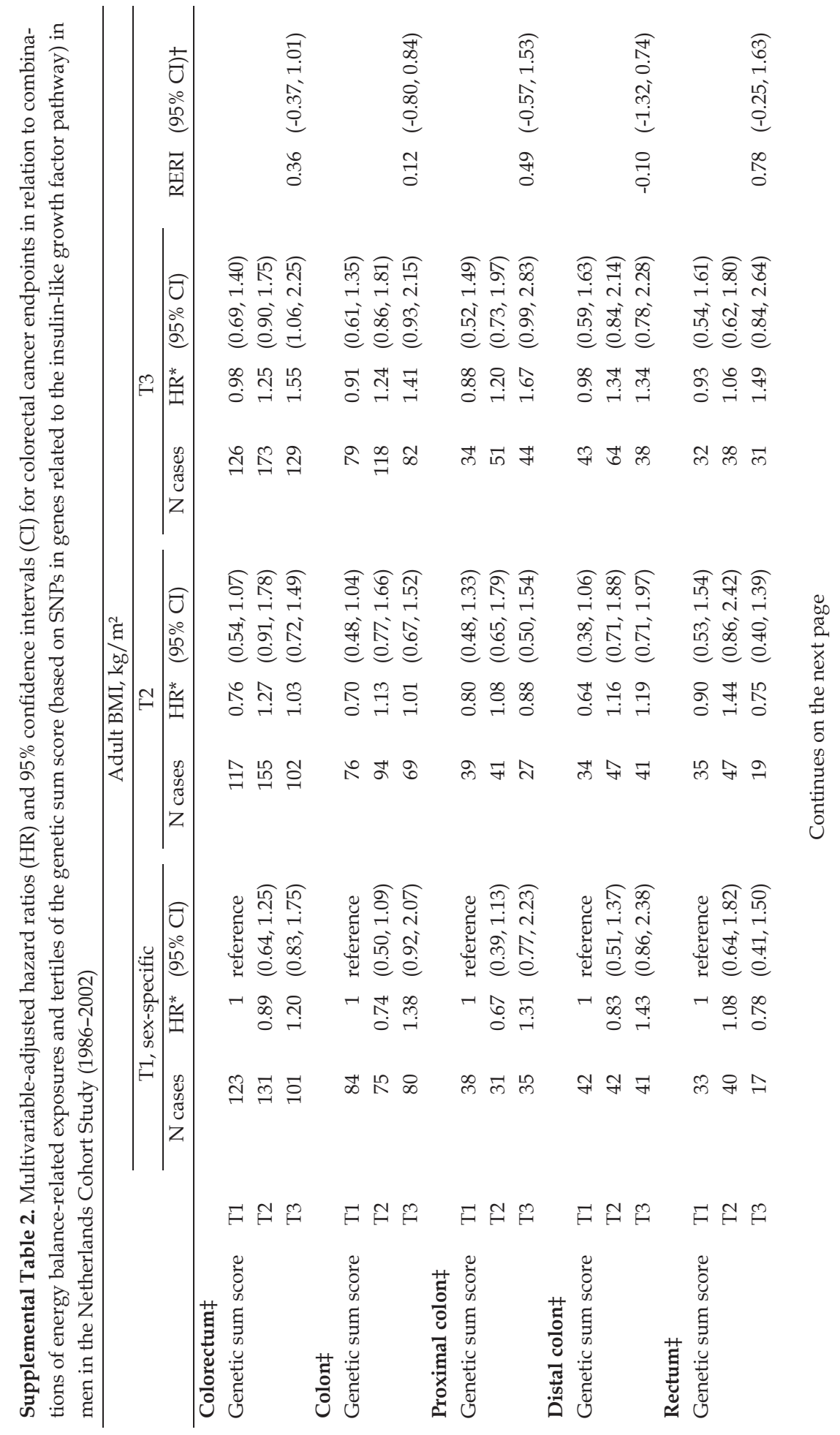




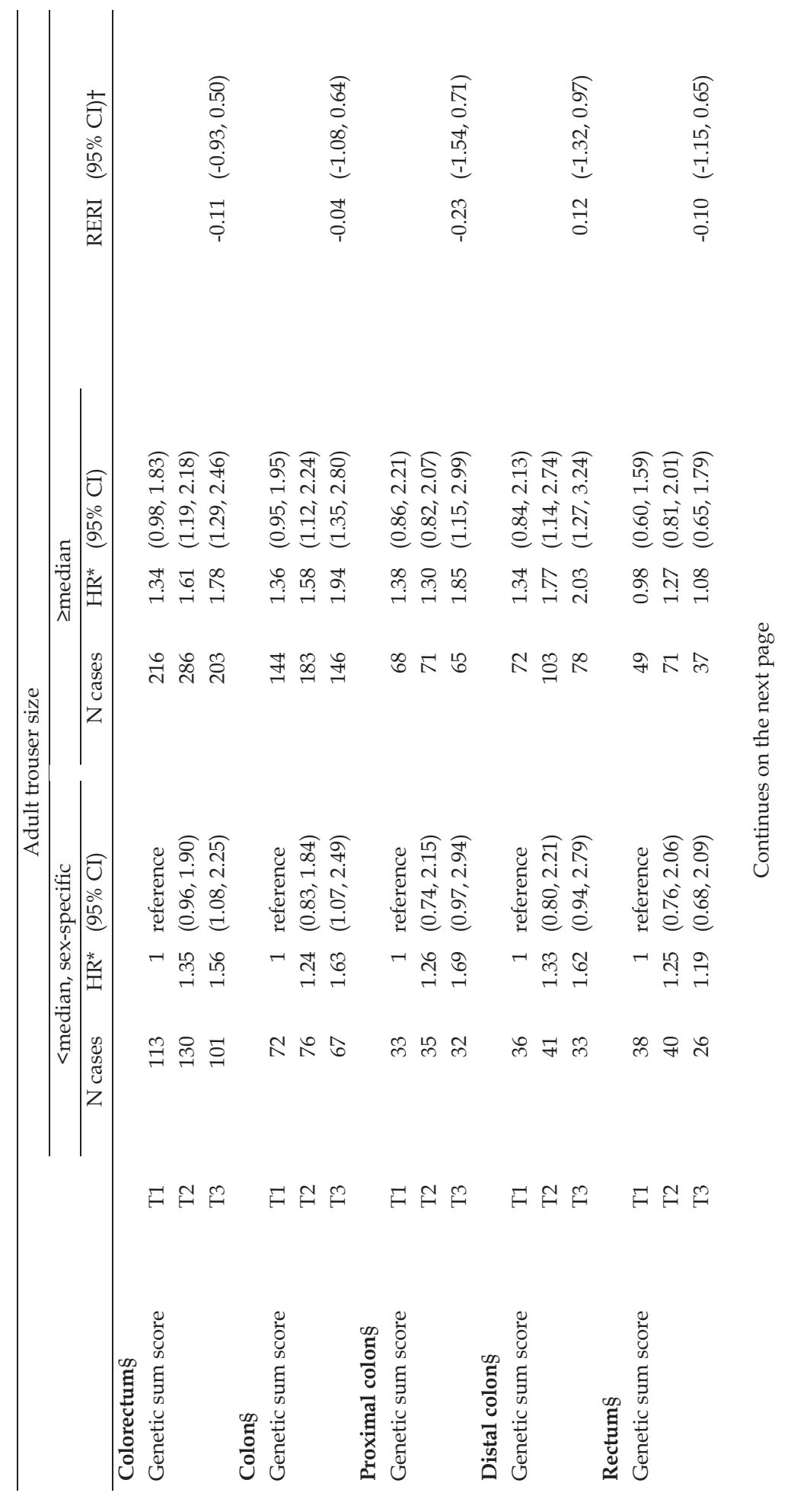




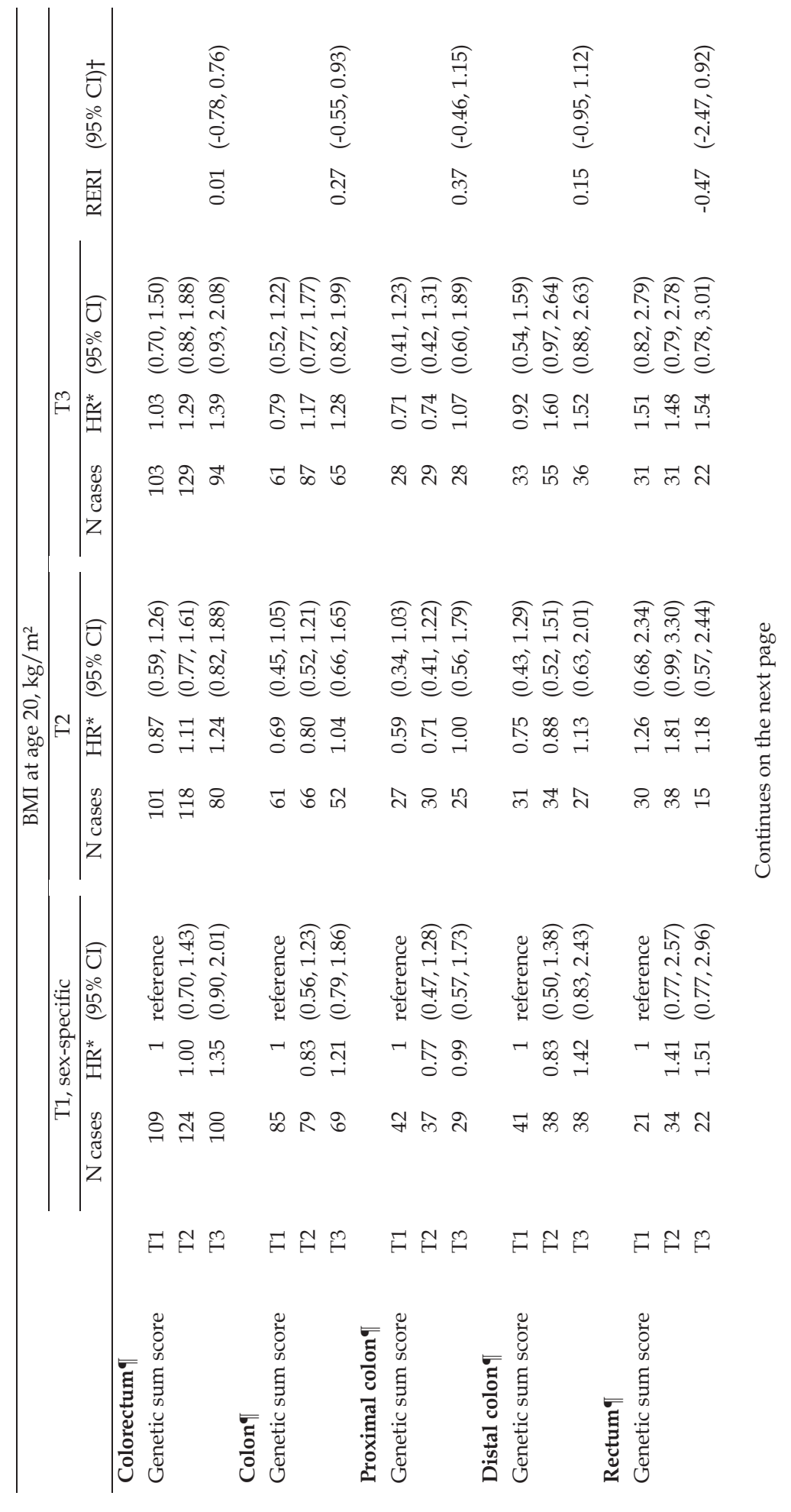




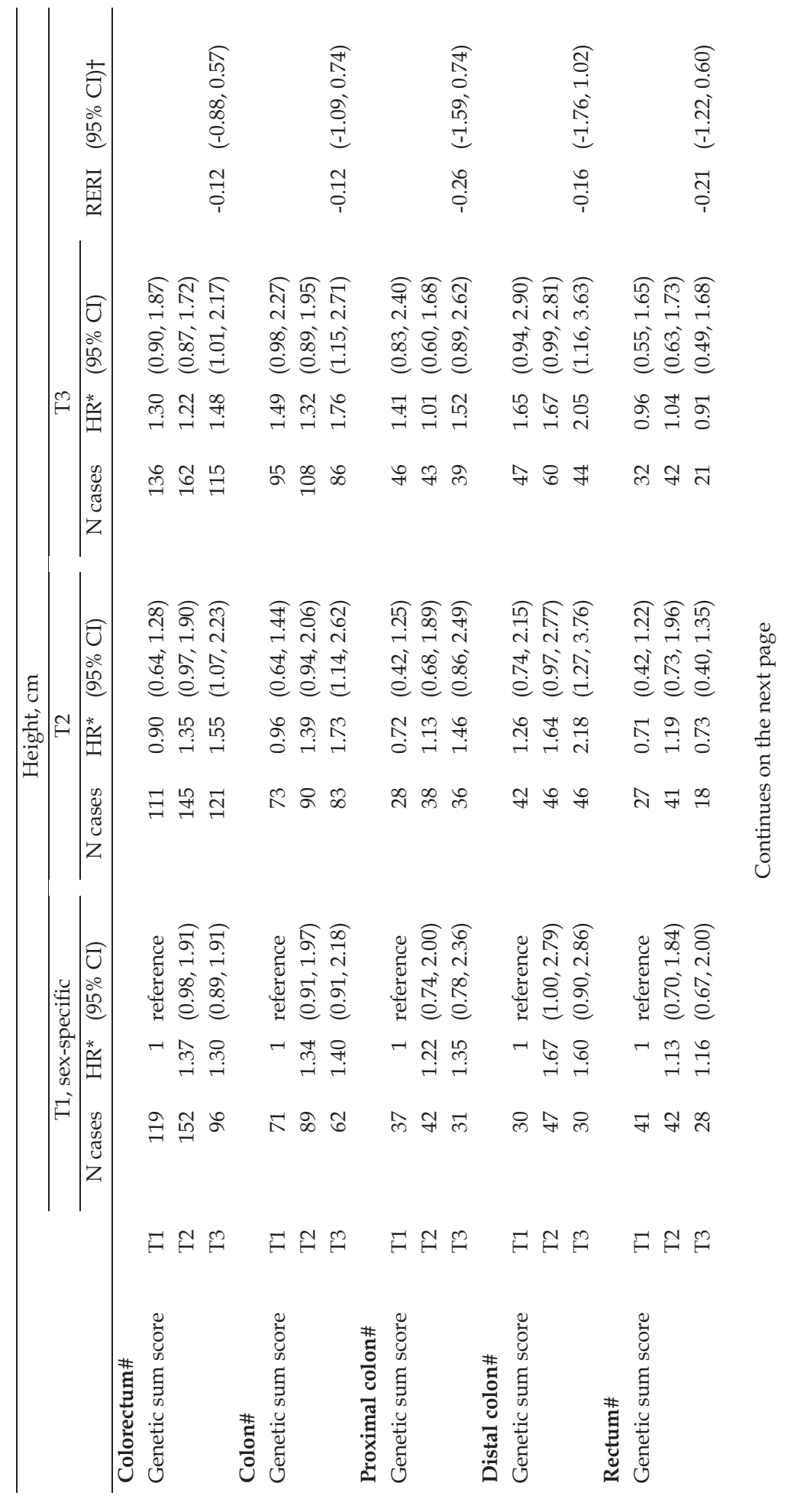




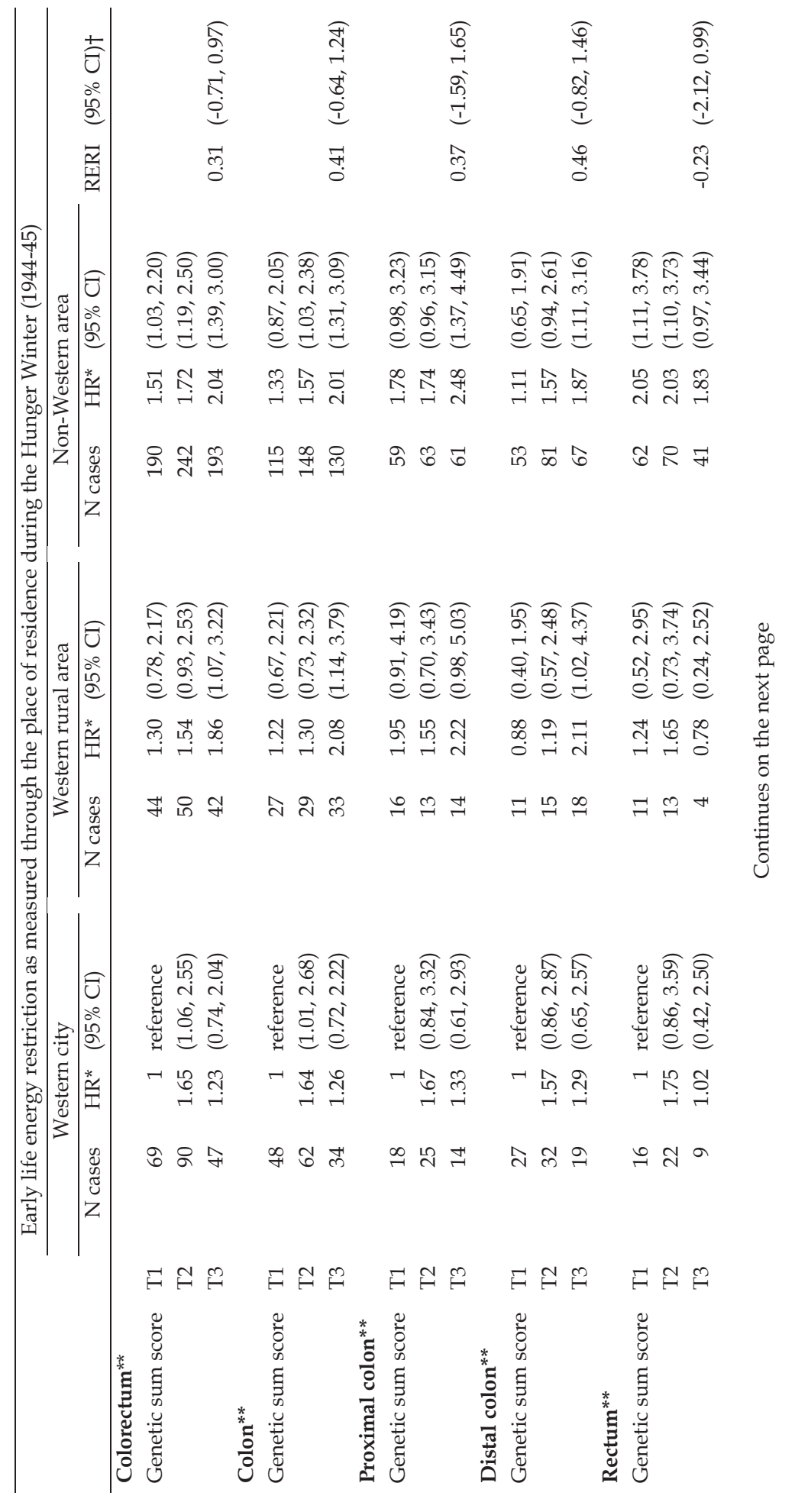




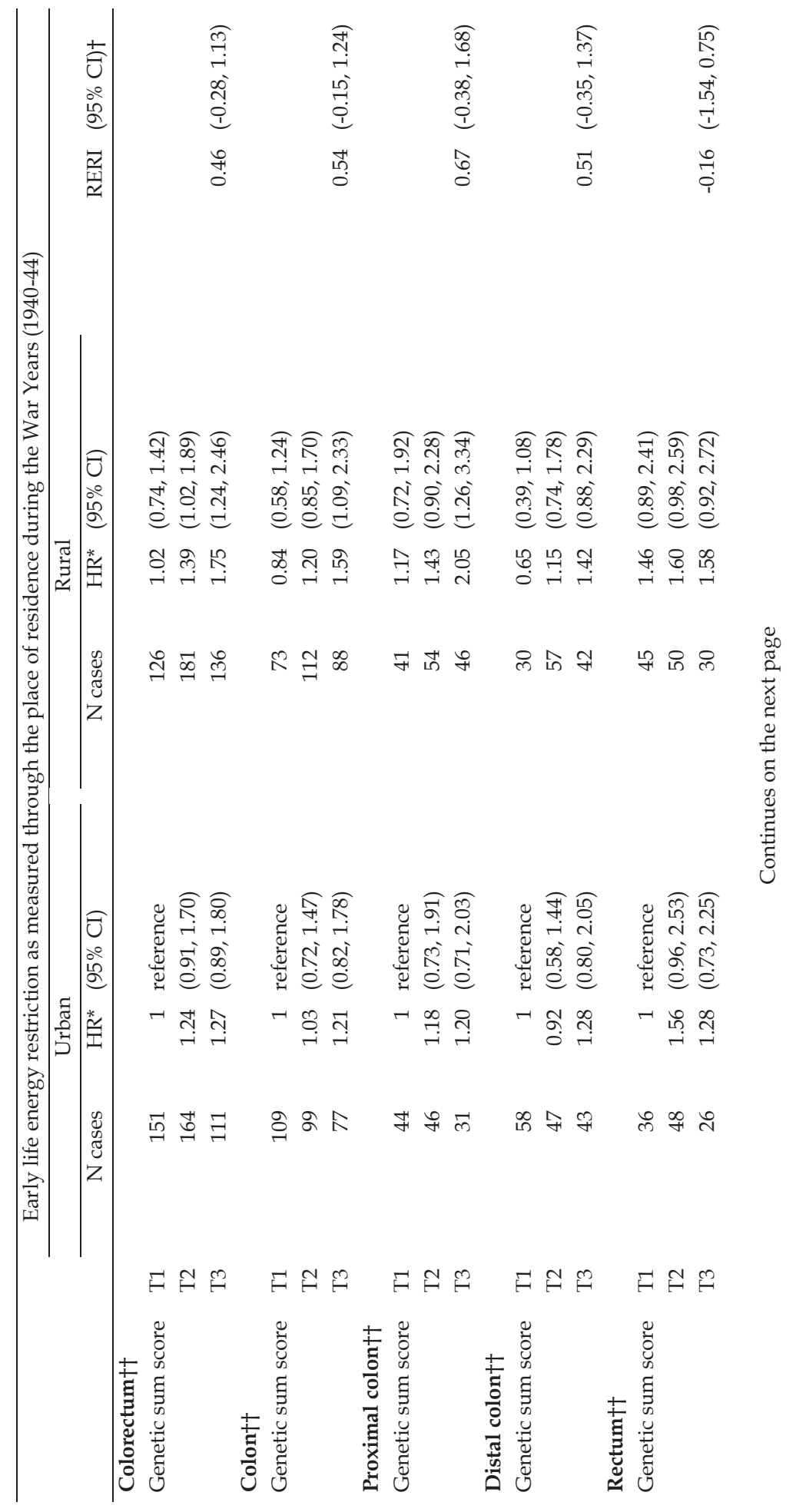




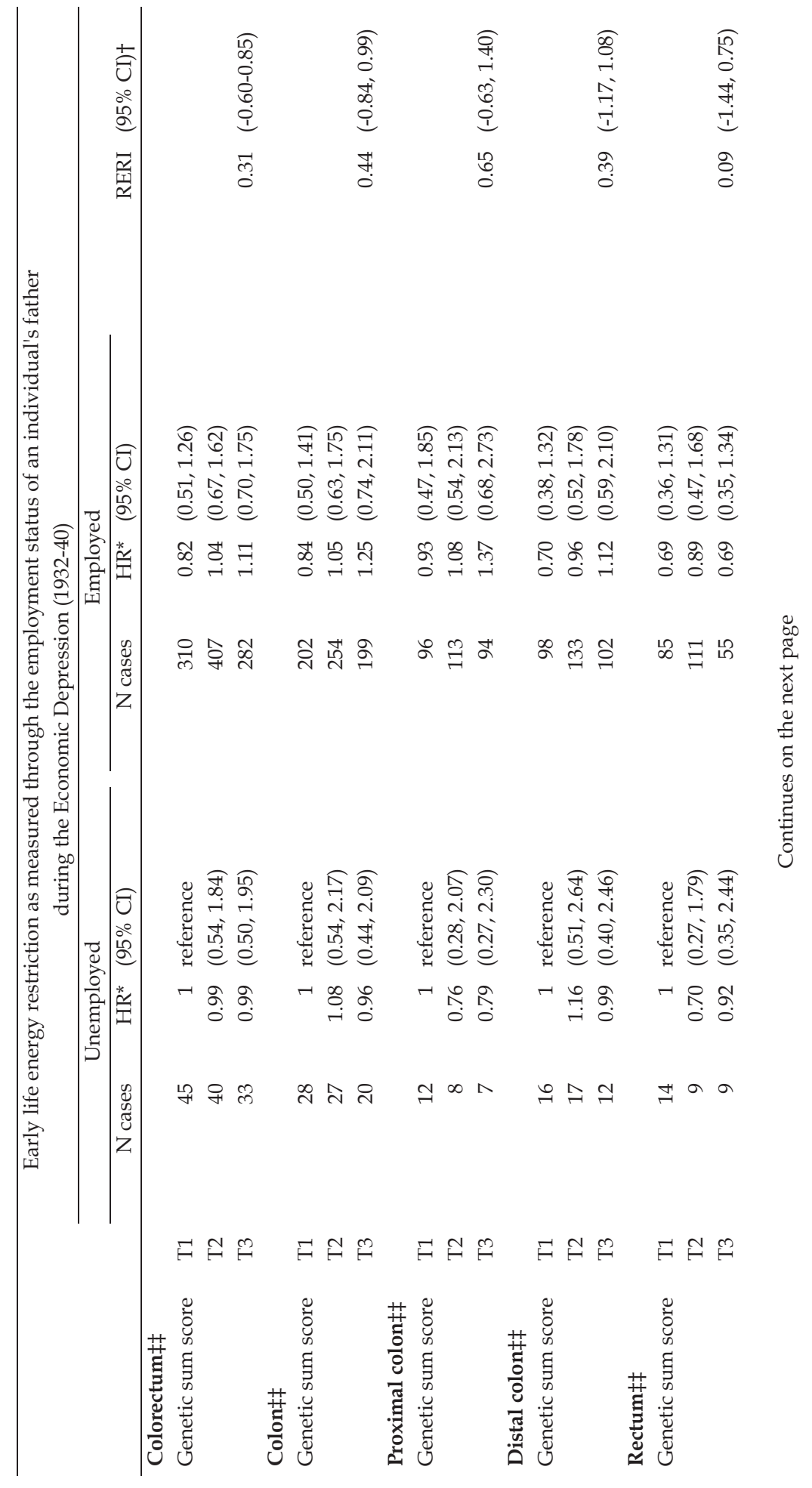




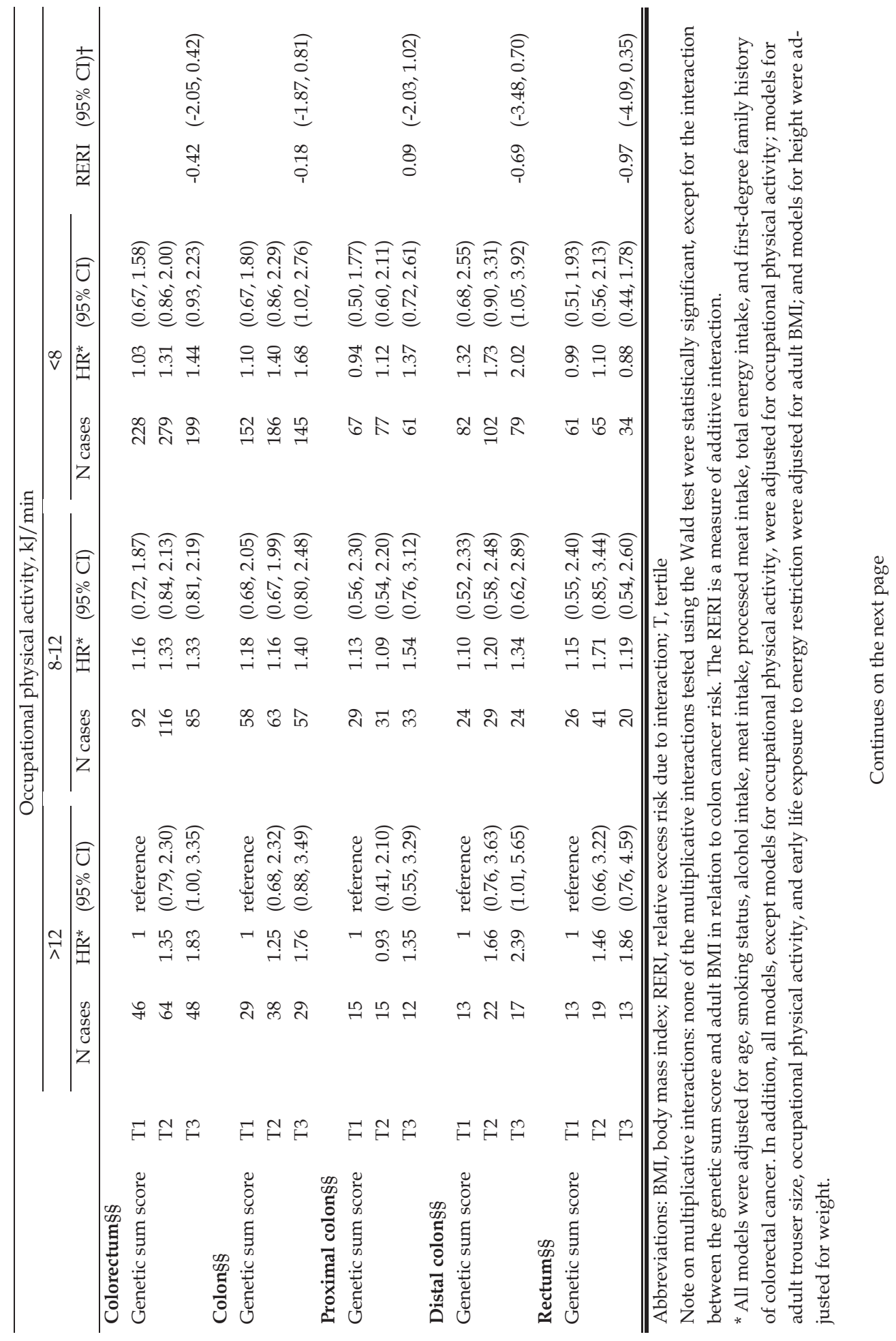




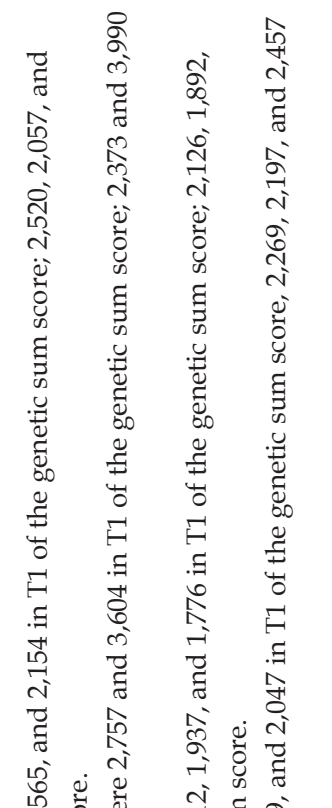

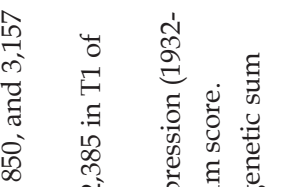

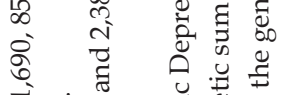

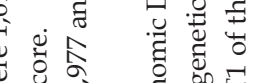

उ

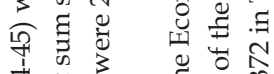

本若尔

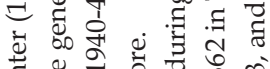

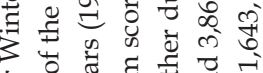

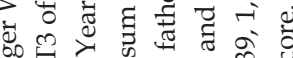

$\Xi \exists \tilde{y}$

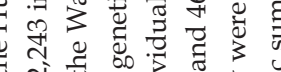

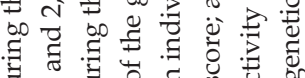

ప స రి

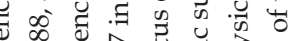

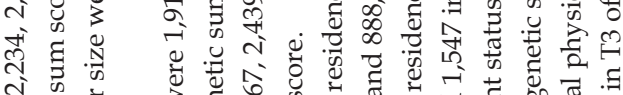

. एँ

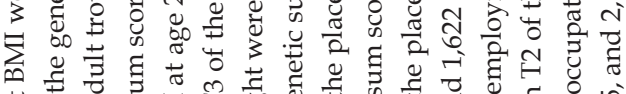

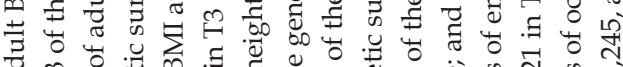

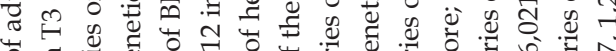
tै.

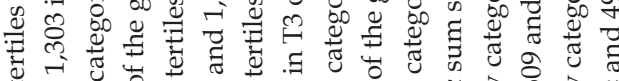
可

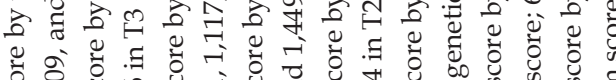

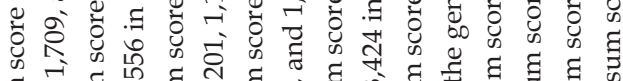

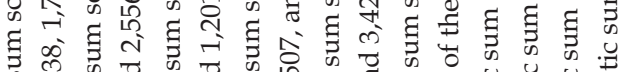

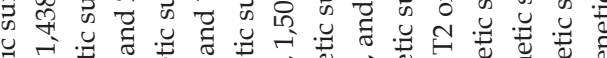

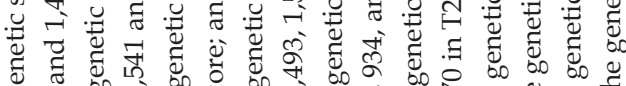

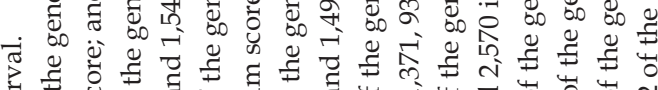
击

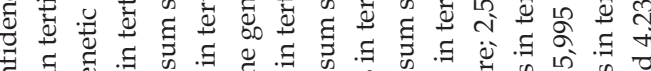

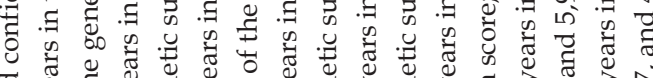

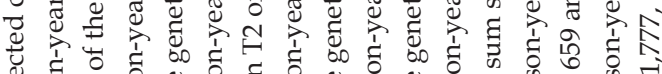

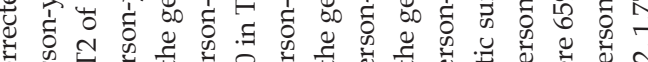
嵌

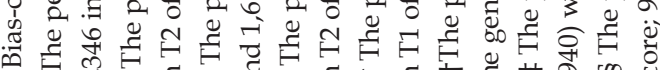
茾策的. 


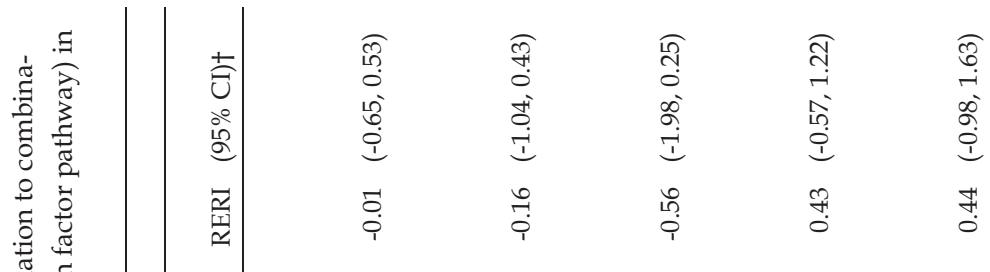

든담

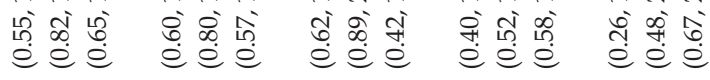

œ

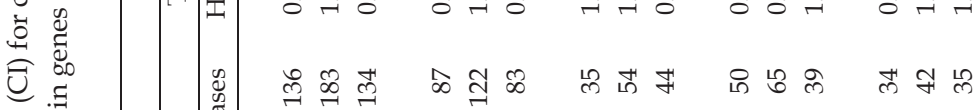

$\frac{\pi}{2}$

苞

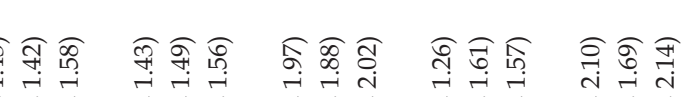

तิ

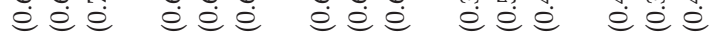

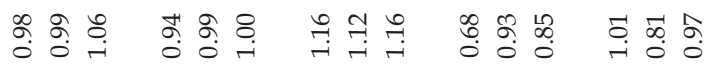

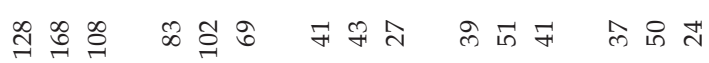

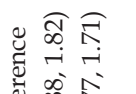

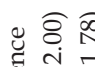

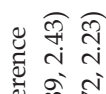

\& 0 o

踏

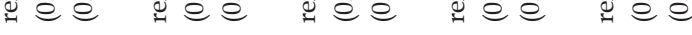

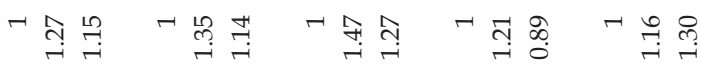

湈

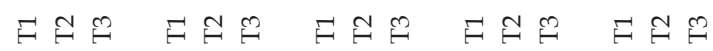




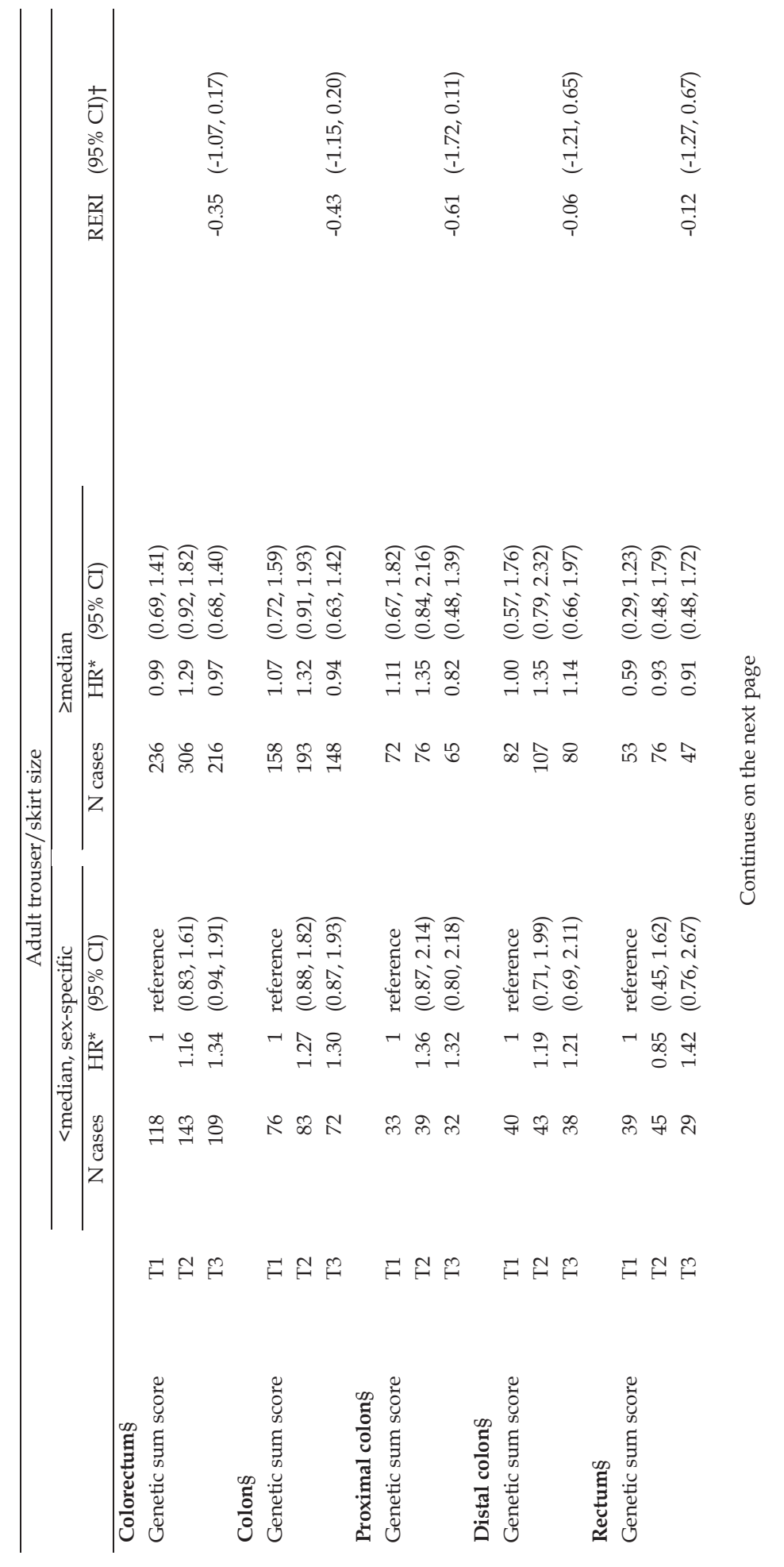




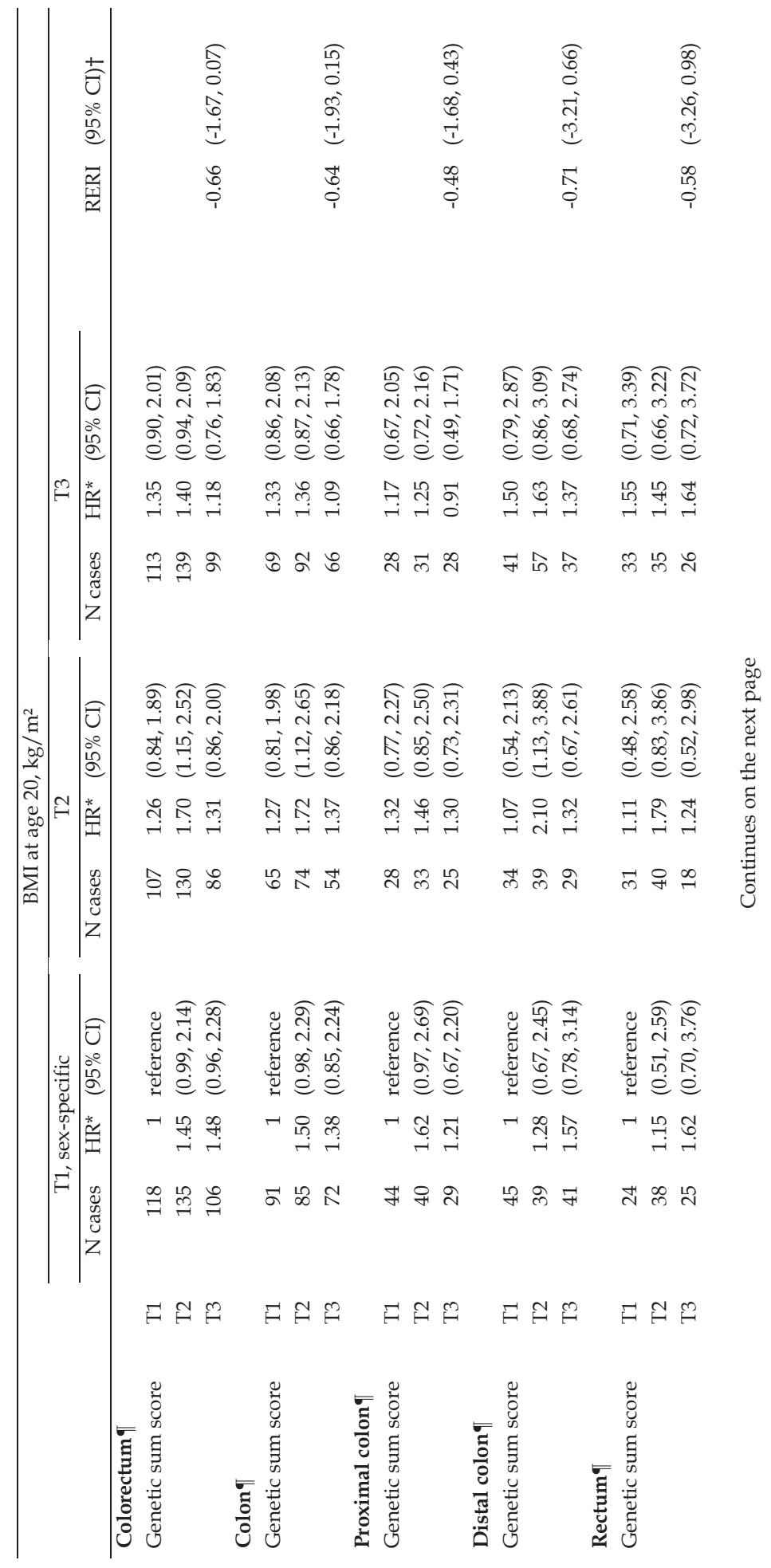




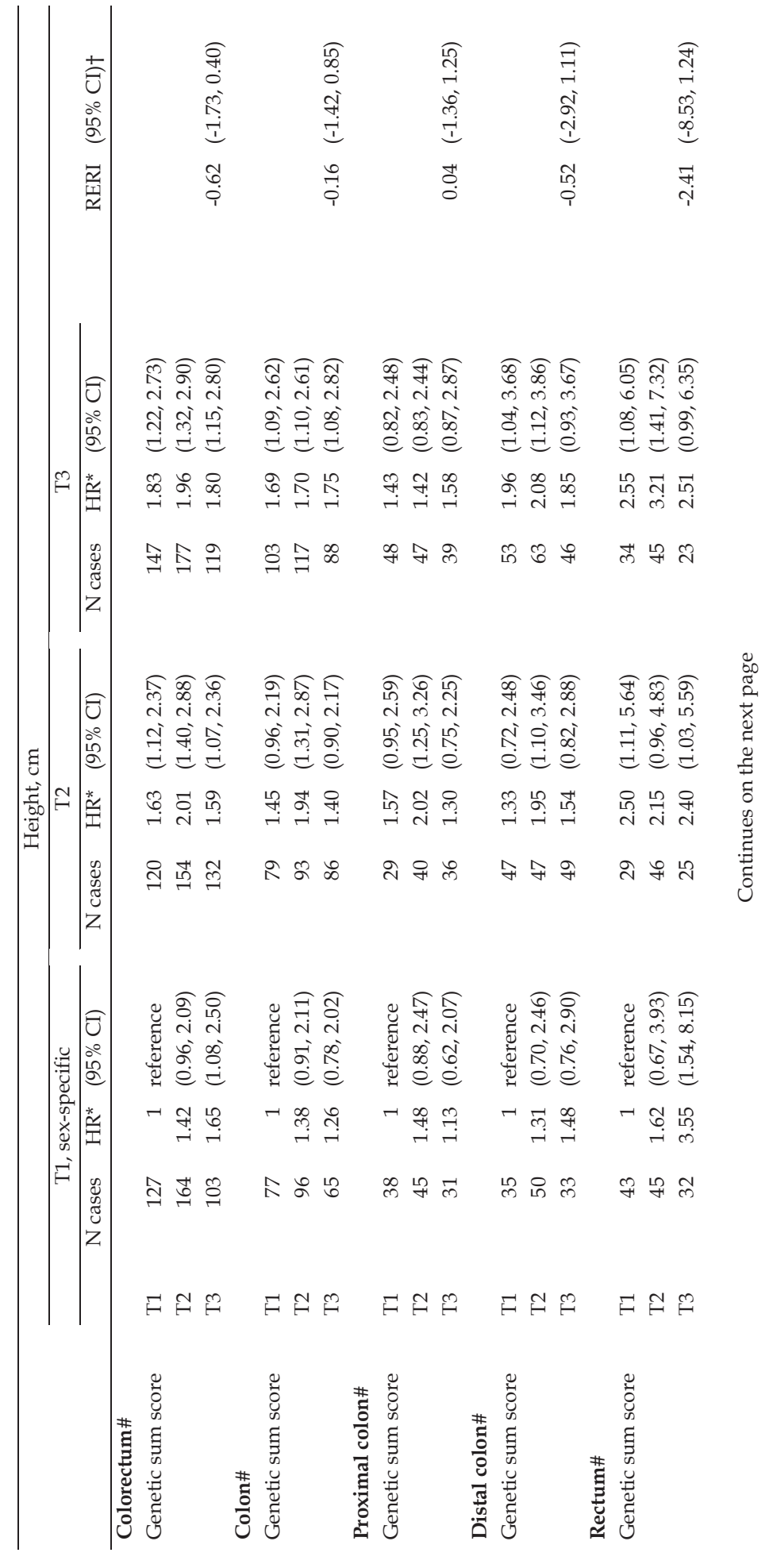




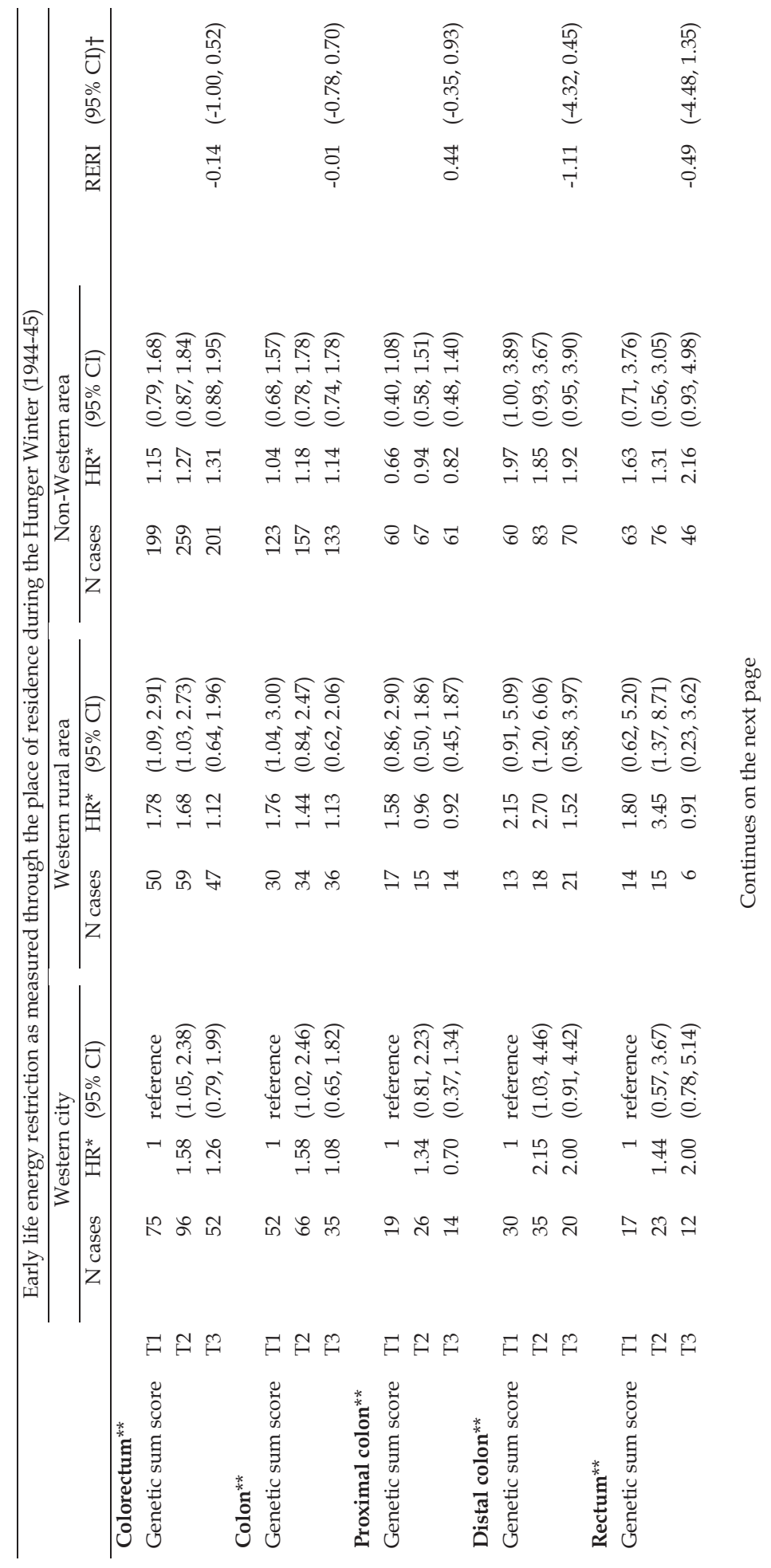




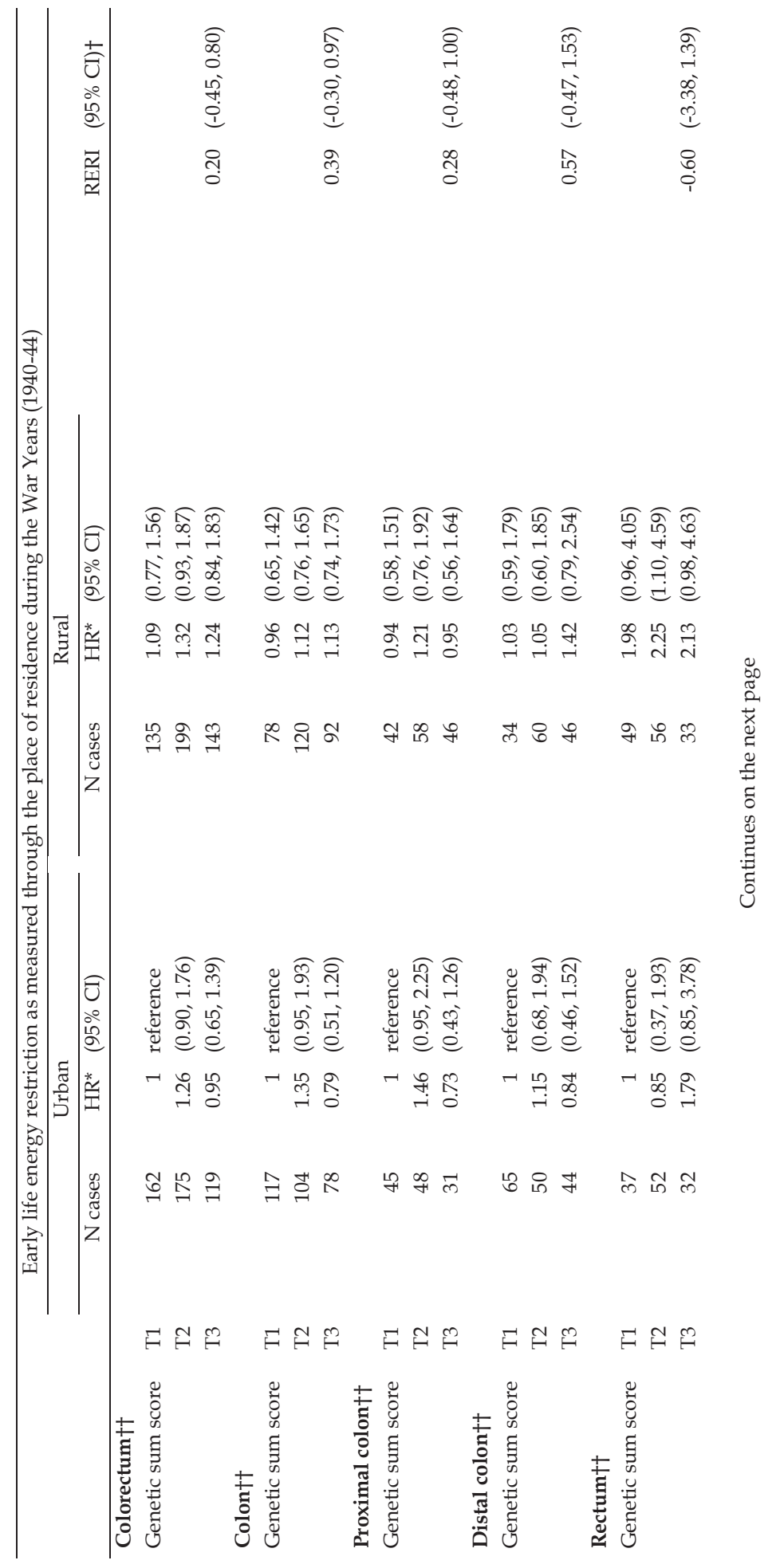




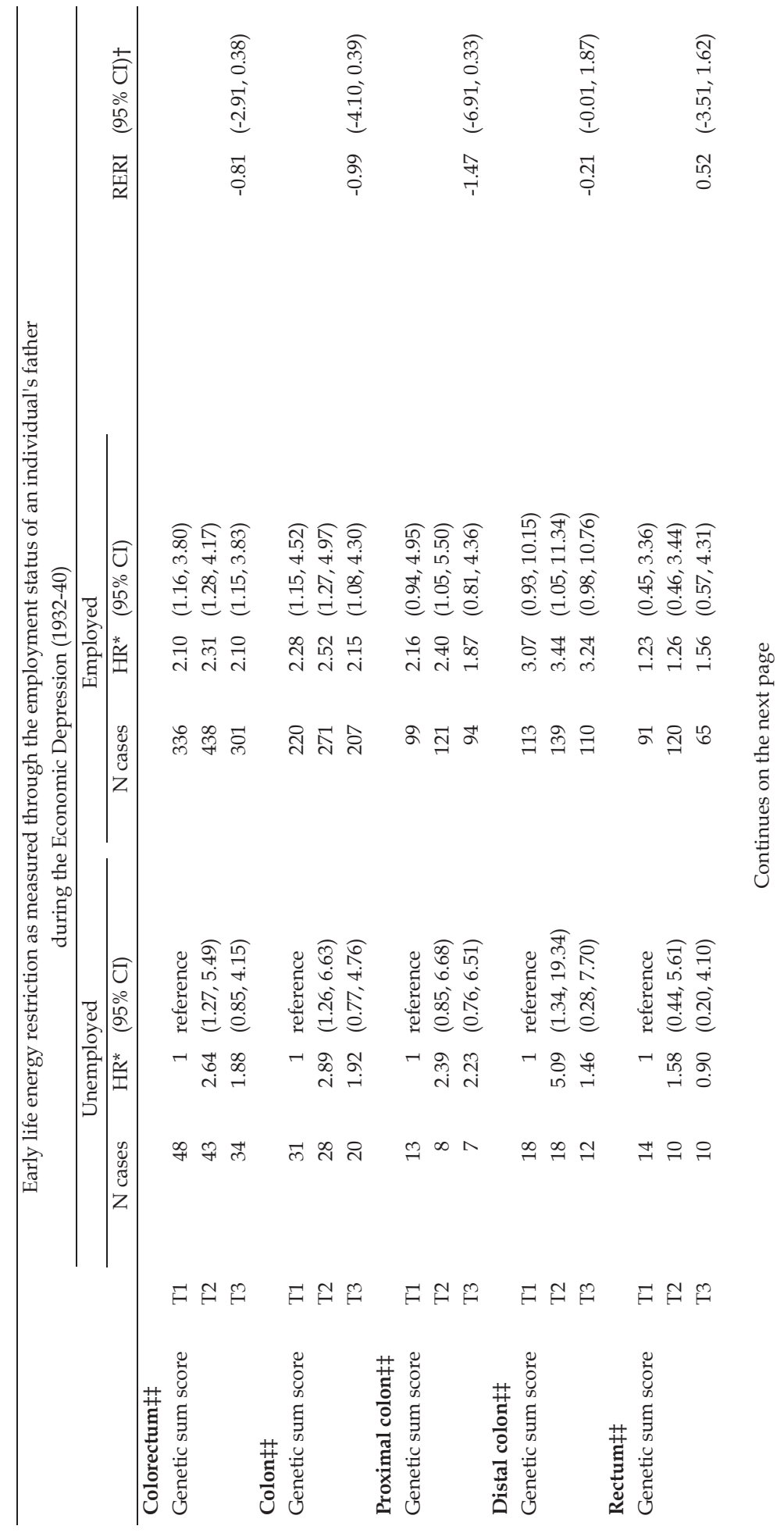




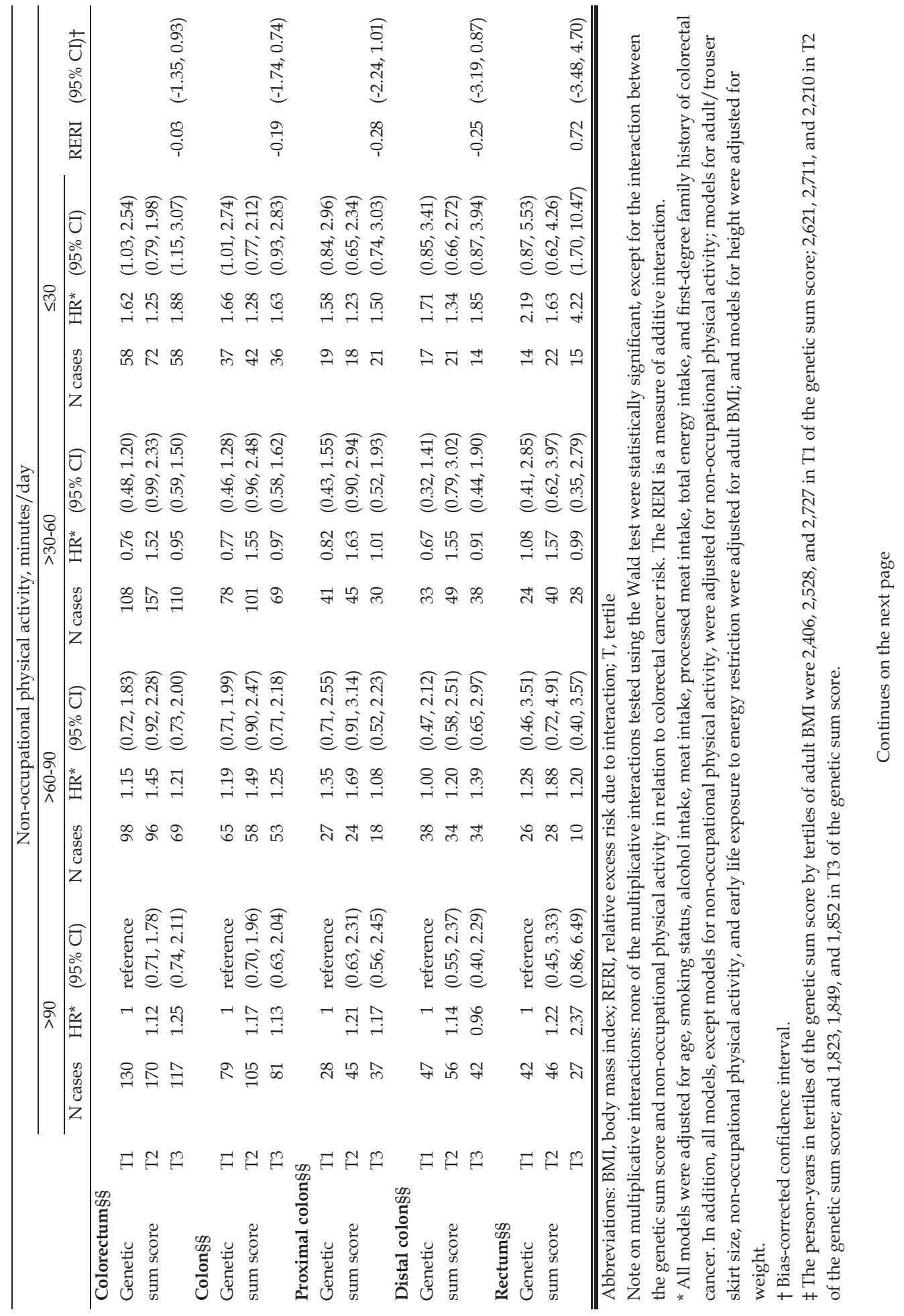




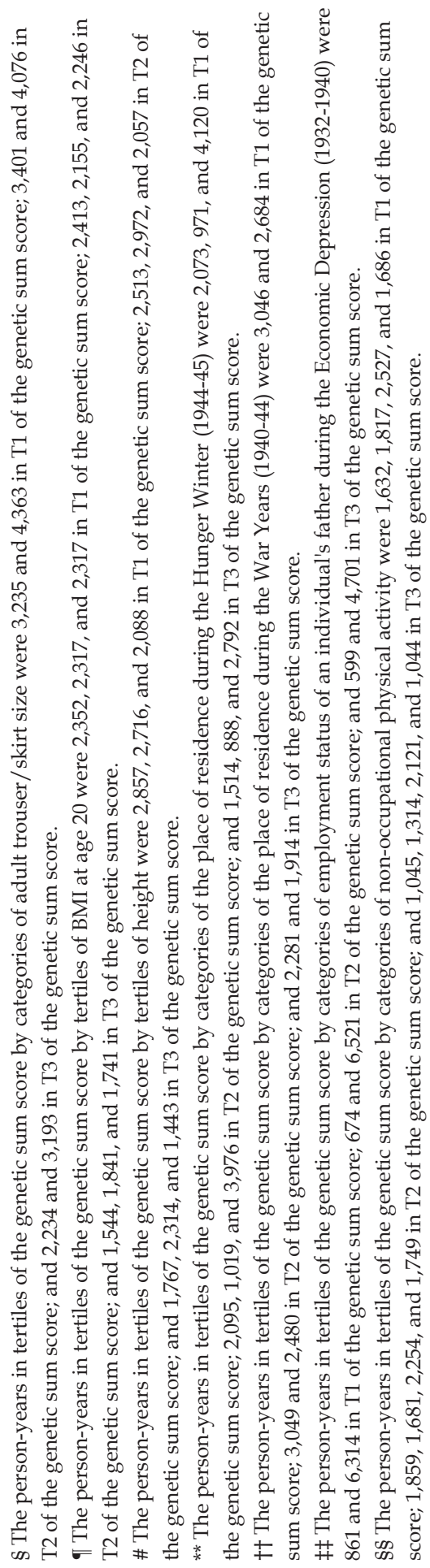


Energy balance influences the risk of having a colorectal tumor with methylated insulin-like growth factor binding protein genes

Colinda CJM Simons, Piet A van den Brandt, Coen DA Stehouwer, Manon van Engeland and Matty P Weijenberg

Submitted for publication 


\section{-Abstract-}

Background: Promoter $\mathrm{CpG}$ island hypermethylation is an early event in colorectal tumorigenesis, potentially silencing genes. We investigated whether methylation of growth-inhibiting insulin-like growth factor binding protein (IGFBP) genes links energy balance-related exposures to colorectal cancer (CRC) risk.

Methods: We determined IGFBP2, IGFBP3, and IGFBP7 promoter CpG island hypermethylation in tumors of 733 CRC cases from the Netherlands Cohort Study $(n=120,852)$. Participants self-reported on body size, physical activity, early life energy restriction, and other factors at baseline. Using a case-cohort approach (n subcohort=5,000), we estimated hazard ratios for CRC by extent of IGFBP methylation.

Results: Comparison of the highest versus lowest sex-specific tertiles of adult body mass index (BMI) gave multivariable-adjusted hazard ratios (95\% confidence intervals (CIs)) for CRCs with $0(18.7 \%), 1(29.5 \%), 2(32.4 \%)$, and $3(19.5 \%)$ methylated genes of $1.39(0.88,2.19), 1.11(0.77$, $1.62), 1.67(1.17,2.38)$ and $2.07(1.29,3.33)$, respectively. Other body size measures and physical activity were not associated with CRC risk by extent of IGFBP methylation. Exposure to early life energy restriction during the Dutch Hunger Winter versus non-exposure gave HRs (95\% CIs) for CRCs with $0,1,2$, and 3 methylated genes of $1.01(0.67,1.53), 1.03(0.74,1.44), 0.72(0.52$, $0.99)$, and $0.50(0.32,0.78)$, respectively; additional proxies corroborated this relationship.

Conclusion: IGFBP methylation linked adult BMI and early life energy restriction to CRC risk. If measurable in early lesions and representative of changes in subsequent lesions, IGFBP methylation might turn out a marker for identifying individuals who may particularly benefit from weight gain prevention. 


\subsection{Introduction}

The insulin-like growth factor (IGF) system, which regulates growth by affecting cell proliferation, differentiation, and apoptosis, has long been hypothesized to link energy balance-related exposures, such as overweight and physical activity, to colorectal cancer (CRC) risk. ${ }^{1,2}$ Silencing of genes through promoter CpG island hypermethylation (in short: methylation) is thought to be an early event in colorectal tumorigenesis. With respect to the IGF system, in particular methylation of insulin-like growth factor binding protein (IGFBP) genes - central components in the IGF system - may sensitize individuals to the effects of energy balance-related exposures on CRC development. This is because IGFBPs generally have a growth-inhibiting function as they sequester IGFs, thereby down-regulating IGF bioavailability. ${ }^{1-3}$ Of the 7 different IGFBPs that exist, IGFBP3 has the highest binding potential. ${ }^{3}$ IGFBP methylation has been indicated an unfavorable event in cancer development, including CRC development, by cell studies investigating methylation of IGFBP $1,{ }^{4}$ IGFBP $2,{ }^{5}$ IGFBP $3,{ }^{4}$ IGFBP $4,{ }^{5}$ and IGFBP $7 .{ }^{6-9}$ Observational evidence is absent.

Any modifying effect of IGFBP methylation on the association between energy balance and CRC risk is difficult to study epidemiologically as methylation is tissue-specific; ${ }^{10}$ the methylation status of genes thus likely differs in normal versus tumor tissue. However, if IGFBP methylation links energy balancerelated exposures to $\mathrm{CRC}$, one would expect these exposures associated with the risk of CRC characterized by IGFBP methylation. We studied this hypothesis within the Netherlands Cohort Study (NLCS) in order to clarify etiological pathways. We also explored the relationship between IGFBP methylation and the CpG island methylator phenotype $(\mathrm{CIMP})^{11}$ and microsatellite instability (MSI), ${ }^{12}$ as these are well-recognized, correlated events in CRC that involve methylation of several tumor suppressor- and DNA repair genes, and are associated with distinct sublocalizations, somatic mutations and etiology. ${ }^{13}$ The characterization of molecular tumor characteristics such as IGFBP methylation may eventually yield future screening markers - if measurable in early lesions and representative of changes in subsequent lesions - for the identification of individuals who may particularly benefit from preventive strategies.

\subsection{Methods}

\subsubsection{Study population and design}

The Netherlands Cohort Study (NLCS) ${ }^{14}$ includes 120,852 participants, sampled from 204 Dutch municipalities, who were 55-69 years old at baseline in 1986. At baseline, participants completed a self-administered questionnaire, including a semi-quantitative 150item food frequency questionnaire (FFQ). The FFQ was found to rank individuals adequately according to dietary intake as compared to a 9-day dietary record, ${ }^{15}$ and was shown a good indicator of intake for at least five years. ${ }^{16,17}$ Participants who reported a history of cancer (other than skin cancer) were excluded. The NLCS was approved by the review boards of the TNO Nutrition and Food Research Institute and Maastricht University in the Netherlands.

The NLCS is characterized by a case-cohort approach. This approach entails that a random subcohort $(n=5,000)$ - selected immediately after baseline and representative of the whole cohort-is followed up to estimate the person-time at risk, whereas incident cancer cases are enumerated for the entire cohort. Subcohort members contribute to the persontime at risk until the end of follow-up, cancer incidence, death or loss to follow-up. Followup for vital status is performed through linkage to the Central Bureau of Genealogy and 
the municipal population registries ( $100 \%$ completeness). Cancer follow-up is performed through linkage with the population-based cancer registry and PALGA (Netherlands pathology database) (more than 96\% completeness). ${ }^{18-20}$ In the period 1989-1993 (the followup period used for the current analyses), 939 incident CRC cases occurred. Sufficient tumor
DNA, isolated from formalin-fixed, paraffinembedded sections after macro-dissection of tumor cells, was available for 733 cases (Figure 1). Age at diagnosis, tumor sublocalization (ICD-O-1 153) and tumor node metastasis (TNM) stage were retrieved from the cancer registry.

Netherlands Cohort Study
(NLCS)
$\mathrm{n}=120,852$
$\begin{aligned} & \text { Baseline questionnaire } \\ & \text { Record linkage to the }\end{aligned}$
$\begin{aligned} & \text { Netherlands Cancer Registry } \\ & \text { and PALGA for cancer } \\ & \text { follow-up }\end{aligned}$
$\begin{aligned} & \text { Record linkage to the Central } \\ & \text { Bureau of Genealogy and the } \\ & \text { municipal population registries } \\ & \text { for follow-up of vital status to } \\ & \text { estimate the person-time at } \\ & \text { risk based on a random } \\ & \text { subcohort (n=5,000) }\end{aligned}$

Figure 1. Flow chart of colorectal cancer cases available for analysis.

Abbreviations: CRC, colorectal cancer; PALGA, Netherlands pathology database * Tumor tissue was collected after approval by the ethical review boards of Maastricht University, the population-based cancer registry, and PALGA; the pathology laboratories made available the tumor blocks between August 1999 and December 2001.

\subsubsection{Energy balance-related exposures}

Adult body mass index (BMI, weight (kg)/height $\left.(\mathrm{m})^{2}\right)$, BMI at age 20, BMI change since age 20 , height $(\mathrm{cm})$, and adult trouser/skirt size were derived from the baseline questionnaire. Adult BMI, BMI at age 20, and height were categorized into sex-specific tertiles. We deviated from the BMI categorization of the World Health Organization (WHO) that distinguishes between underweight $\left(<18.5 \mathrm{~kg} / \mathrm{m}^{2}\right)$, normal weight $(18.5-<25$ $\left.\mathrm{kg} / \mathrm{m}^{2}\right)$, overweight $\left(25-<30 \mathrm{~kg} / \mathrm{m}^{2}\right)$, and obese individuals $\left(\geq 30 \mathrm{~kg} / \mathrm{m}^{2}\right),{ }^{21}$ because of power considerations seeing that $92.3 \%$ of subcohort members fell within the normal adult weight range or overweight range. At age $20,92.3 \%$ of subcohort members fell below the overweight range. Adult trouser/skirt size was shown to correlate well with hip and waist circumferences in a subset of weightstable individuals, and was associated with endometrial and renal cell cancer risk in a fashion as would be expected for waist circumference, rendering it a good proxy.22

Non-occupational physical activity at baseline in minutes per day was a sum measure of several activities. It included daily walking/cycling ( $\mathrm{min} /$ day), weekly recreational walking/cycling, weekly engagement in gardening/odd jobs, and weekly participation in sports/gymnastics (categories: never, 1, 1-2, and $>2$ hours/week). Categories were $\leq 30$, 
$>30-60,>60-90$, and >90 minutes per day. In the present paper, the two middle categories were combined for power considerations. Occupational physical activity was derived from self-reported occupational history on the basis of a rating system developed by Hettinger et al. ${ }^{23}$ which distinguishes between jobs with an energy expenditure of $<8,8-12$, and $>12$ $\mathrm{kJ} / \mathrm{min}$.

Early life energy restriction was measured through three proxy variables: the place of residence during the Hunger Winter (194445); the place of residence in 1942, reflecting the War Years (1940-44); and the employment status of an individual's father during the Economic Depression (1932-40). Nutritional differences during these periods have been well-documented in the Netherlands. In particular living in a Western city in the Netherlands during the Hunger Winter indicated severe energy restriction. The Dutch Hunger Winter was preceded by a German food embargo and was unusually early and harsh; by the time the embargo was (partially) lifted, the canals had frozen over, which made it impossible to transport food into the Western parts of the country, and 11 cities are considered famine cities: Amsterdam, Rotterdam, The Hague, Utrecht, Zaandam, Hilversum, Amersfoort, Dordrecht, Vlaardingen/Schiedam, Delft, and Leiden. At the height of the famine, from December 1944 to April 1945 , official daily rations per capita were between 400 and 800 kilocalories, although the diet remained nutritionally balanced. ${ }^{24,25}$ During the Economic Depression, sufficient calories were available, but the variation in the food pattern had likely been limited if an individual's father was unemployed. NLCS participants were between 12-28 years old during the Hunger Winter, between 8-28 years old during the War Years, and between $0-23$ years old during the Economic Depression.
Data on adult BMI, BMI at age 20, BMI change, adult trouser/skirt size, height, nonoccupational physical activity, occupational physical activity, place of residence during the Hunger Winter, place of residence during the War Years, and employment status of an individual's father during the Economic Depression were complete for $96.1 \%, 82.0 \%, 81.8 \%$, $93.4 \%, 96.7 \%, 98.0 \%, 87.1 \%, 87.3 \%, 72.6 \%$, and $93.9 \%$ of subcohort members, respectively.

\subsubsection{Laboratory analyses}

IGFBP methylation. We determined IGFBP2, IGFBP3, IGFBP4, and IGFBP7 CpG island promoter hypermethylation by methylationspecific PCR (MSP) 26,27 after bisulfite modification of 500 ng DNA (Zymo Research), because methylation had been reported in these IGFBP genes. To facilitate the methylation-specific PCR (MSP) on DNA retrieved from formalinfixed, paraffin-embedded tissue, DNA was first amplified with flanking PCR primers that amplify bisulfite-modified DNA but do not preferentially amplify methylated or unmethylated DNA. The resulting fragment was used as a template for the MSP reaction. All PCRs were carried out with controls for unmethylated alleles (DNA from normal lymphocytes), methylated alleles (normal lymphocyte DNA treated in vitro with SssI methyltransferase (New England Biolabs, lpswich, MA)), and a control without DNA. Ten microlitres of each MSP reaction was directly loaded on to a nondenaturing $2 \%$ agarose gel, stained with gelstar and visualized under UV illumination. Primers were designed on the basis of a deep sequencing analysis of methylation in colon cancer cell lines, in order to precisely cover densely methylated CpG islands. ${ }^{10}$ IGFBP4 methylation analyses were discontinued after preliminary results showed methylation in only four out of 100 samples. We concluded that IGFBP4 methylation is rare in CRC and that analyses would not be cost-effective. The pri- 
mer sequences used to analyze IGFBP2, IGFBP 3 and IGFBP7 are shown in Table 1. Methylation analyses of IGFBP2, IGFBP3, and IGFBP7 were successful in $98.9 \%, 94.4 \%$, and $98.0 \%$ of 733 CRC cases, respectively; repro- ducibility in duplo or triplo analyses was 93.2\% $(\mathrm{n}=103$ sample pairs $/$ trios $), 95.1 \%(\mathrm{n}=$ 122 sample pairs/trios), and $84.4 \%(\mathrm{n}=77$ sample pairs/trios), respectively.

Table 1. Primer sequences used to analyze CpG island promoter hypermethylation of IGFBP2, IGFBP3, and IGFBP7 in colorectal tumors

\begin{tabular}{|c|c|}
\hline & Primer sequence \\
\hline \multicolumn{2}{|l|}{ IGFBP2 } \\
\hline Flanking primer, upstream & GTTTAGTGTYGGTTATAGGGGAAG \\
\hline Flanking primer, downstream & ACTCСТАAАAАAAAATACRAATAACTC \\
\hline Methylated sense strand & GAAGCGCGTAAACGAAGTTTTC \\
\hline Methylated antisense strand & CGACGTAACGATAAAAAAAAACG \\
\hline Unmethylated sense strand & GGGGAAGTGTGTAAATGAAGTTTTT \\
\hline Unmethylated antisense strand & AAACCAACATAACAATAAAAAAAAACA \\
\hline \multicolumn{2}{|l|}{ IGFBP3 } \\
\hline Flanking primer, upstream & TTTTTTTTAATTTTTATTTTTGGG \\
\hline Flanking primer, downstream & AAACAAAAAACRACTAATCCTCAAC \\
\hline Methylated sense strand & TTTTTATTTTTGGGCGCGTC \\
\hline Methylated antisense strand & GCCCAACCGCAATACTCG \\
\hline Unmethylated sense strand & TTTTTAATTTTTATTTTTGGGTGTGTT \\
\hline Unmethylated antisense strand & CТСААСАСССААССАСААТАСТСА \\
\hline \multicolumn{2}{|l|}{ IGFBP7 } \\
\hline Flanking primer, upstream & TTATTATTTAGGTTAGTAAGGGTATTTG \\
\hline Flanking primer, downstream & СССАТСТААСТССТААААТАССС \\
\hline Methylated sense strand & GCGAGTAAGGTGGGTACGAGC \\
\hline Methylated antisense strand & GCAATAAACAACGCGAAACG \\
\hline Unmethylated sense strand & GTATTTGTGAGTAAGGTGGGTATGAGT \\
\hline Unmethylated antisense strand & AAAATCACAATAAACAACACAAAACA \\
\hline
\end{tabular}

CIMP and MSI. CIMP was defined by CpG island promoter hypermethylation of $\geq 3$ out of five Weisenberger markers (CACNA1G, IGF2, NEUROG1, RUNX3, and SOCS1). ${ }^{28}$ Methylation of these markers was analyzed using MSP as described previously. ${ }^{29}$ Analyses were successful in $81 \%, 79 \%, 79 \%, 90 \%$, and $83 \%$ of 733 cases for CACNA1G, IGF2, NEUROG1, RUNX3, and SOCS1, respectively. MSI was determined by common approach: a pentaplex PCR using the mononucleotide repeats BAT-26, BAT-25, NR-21, NR-22, and NR-24. Allelic size variations in $\geq 3$ repeats were a marker for MSI; other tumors were classified as microsatellite stable (MSS). ${ }^{30}$ Analyses were successful in $90 \%$ of 733 cases.

\subsubsection{Statistical analysis}

We used Cox regression to estimate hazard ratios and 95\% confidence intervals for CRC risk by extent of IGFBP methylation in relation to energy balance-related exposures using Stata (Stata Corp., College Station, TX). We also performed sex-specific analyses, even though the power to detect associations was limited, because previous NLCS data regarding $\mathrm{CRC}$ risk showed heterogeneous results 
for men and women: indicators of body fat, 31 occupational physical activity, 32 and early life energy restriction ${ }^{33}$ were associated with risk in men, whereas height ${ }^{31}$ and non-

occupational physical activity ${ }^{32}$ were associated with risk in women. With respect to physical activity, we modeled non-occupational physical activity and additionally analyzed occupational physical activity in sex-specific analyses for men. Occupational physical activity is not representative of physical activity in women because a substantial number of women were never employed or only in the distant past. The risk of CRC by methylation status of IGFBP2, IGFBP3, and IGFBP7 was estimated for future literature comparisons. To account for the additional variance introduced by sampling the subcohort from the entire cohort, standard errors were estimated using the robust Huber-White sandwich estimator. ${ }^{34}$ A $P$-value $<0.05$ for two-sided testing indicated statistical significance.

We modeled risk with an age- and sexadjusted model, and a model in which we additionally adjusted for the following predefined potential confounders: all models, except models for (non-)occupational physical activity, were adjusted for non-occupational physical activity; models for adult trouser/skirt size, (non-)occupational physical activity, and early life energy restriction were adjusted for adult BMI; and models for height were adjusted for adult weight. Other potential confounders were included if these changed hazard ratios by more than $10 \%$. None of the variables considered did (family history of CRC, smoking status, socioeconomic status, diabetes, total energy intake, and intake of alcohol, meat, processed meat, fruit, vegetables, fiber, fat (energy-adjusted), water through foods and fluids, supplements, folate, beta-carotene, vitamin B6, vitamin C, vitamin E, calcium, iron, magnesium, flavonoids, and catechins). Furthermore, BMI change since age 20 was modeled within strata of adult BMI $\left(<25\right.$ and $\left.\geq 25 \mathrm{~kg} / \mathrm{m}^{2}\right)$ in an effort to disentangle the potential effect of weight gain on CRC risk from that of being overweight or obese in adulthood (BMI change correlated with adult BMI in subcohort members, Pearson $r=0.68$, $P$-value $<0.001)$. We excluded individuals with a negative BMI change in this analysis, as some weight gain with age may be expected, and (severe) weight loss could indicate preclinical disease (the mean BMI change in subcohort members was $3.5 \mathrm{~kg} / \mathrm{m}^{2}$ with a standard deviation of $3.4 \mathrm{~kg} / \mathrm{m}^{2}$ ). Finally, in sensitivity analyses, models for adult BMI and BMI at age 20 were mutually adjusted to check for independent effects; and models for early life energy restriction were additionally adjusted for BMI at age 20 as BMI at age 20 could be an intermediate factor. BMI at age 20 significantly differed between Western and rural areas during the Hunger Winter and War Years in subcohort members who had not reached 20 years of age in 1944 and 1940, respectively $(P$ values for Kruskal-Wallis test $<0.01)$. None had reached 20 years of age at the beginning of the Economic Depression; there was no difference in BMI at age 20 between subcohort members with or without an employed father during this period ( $P$-value Kruskal-Wallis test $=0.66$ ). The proportional hazards assumption was tested using the scaled Schoenfeld residuals and by visually inspecting the -loglog transformed hazard curves. No violations were detected.

\subsection{Results}

\subsubsection{Population characteristics}

Methylation of IGFBP genes was successfully analyzed in 652 CRC cases. The prevalence of IGFBP2, IGFBP3, and IGFBP7 methylation was $40.6 \%(\mathrm{n}=265), 40.2 \%(\mathrm{n}=262)$, and $71.8 \%(\mathrm{n}$ =468), respectively. An IGFBP methylation index showed that $18.7 \%(\mathrm{n}=122)$ of CRC cases had 0 methylated genes, $29.5 \%(n=192)$ had 1 methylated gene, $32.4 \%(n=211)$ had 2 
methylated genes, and 19.5\% $(n=127)$ had 3 methylated genes. Subcohort members and CRC cases did not evidently differ regarding the investigated exposures (Table 2).

The distribution of CRC cases across the IGFBP methylation index significantly differed from that across instability types as based on CIMP and MSI status $(P<0.001)$ (Table 3). The percentage of MSI tumors (of which $76.9 \%$ also had CIMP) and MSS CIMP tumors increased with an increasing number of methylated IGFBP genes $(+17.4 \%$ and $+36.3 \%$, respectively), whereas the percentage of MSS non-CIMP tumors decreased across IGFBP index groups (-53.7\%). All three instability groups were present among CRCs with 3 methylated IGFBP genes: $24.5 \%$ of CRC cases were MSI tumors, $38.7 \%$ were MSS CIMP tumors, and $36.8 \%$ were MSS non-CIMP tumors. With increasing methylated IGFBP gene numbers, the tumor location was more often proximal and less often distal $(P<0.001)$. Age at diagnosis and TNM stage did not significantly differ by extent of IGFBP methylation ( $P=0.98$ and 0.08 , respectively).

5.3.2 CRC risk by extent of IGFBP methylation

Adult BMI and early life energy restriction were associated with CRCs with an increased number of methylated IGFBP genes after multivariable corrections (Table 4). For adult BMI, comparison of the highest versus lowest sexspecific tertiles gave hazard ratios $(95 \%$ confidence intervals (CIs)) for CRCs with 0, 1, 2, and 3 methylated genes of $1.39(0.88,2.19)$, $1.11(0.77,1.62), 1.67(1.17,2.38)$, and 2.07 $(1.29,3.33)$, respectively. Significant linear trends were observed in associations between adult BMI and CRCs with 2 and 3 methylated genes $(P$-values $<0.01)$. Adult BMI modeled per 5-unit increase corroborated the observed associations. BMI at age 20, BMI change since age 20 modeled in strata of adult BMI, height, adult trouser/skirt size, and non-occupational physical activity were not associated with CRC risk by IGFBP methylation status, although there was a borderline significant inverse trend across non-occupational physical activity categories in relation to CRC with 3 methylated IGFBP genes $(P$-trend $=0.06)$. Exposure to early life energy restriction during the Hunger Winter versus non-exposure gave hazard ratios (95\% CIs) for CRCs with 0, 1, 2, and 3 methylated IGFBP genes of 1.01 ( 0.67 , $1.53), 1.03(0.74,1.44), 0.72(0.52,0.99)$, and $0.50(0.32,0.78)$, respectively. A similarly decreased hazard ratio for CRC with 3 methylated IGFBP genes was observed in those exposed to energy restriction during the War Years versus those not exposed (hazard ratio $(H R)=0.59,95 \%$ CI: 0.39, 0.89). Having had an unemployed father during the Economic Depression was not significantly associated with any of the endpoints, yet strong inverse hazard ratios were observed for CRCs with 2 and 3 methylated IGFBP genes $(\mathrm{HR}=0.59$, 95\% CI: 0.35, 1.02 and HR $=0.59,95 \%$ CI: 0.30, 1.19 , respectively). Additional adjustment for adult BMI in models for BMI at age 20 and vice versa did not essentially change results, nor did additional adjustment for BMI at age 20 in models for early life energy restriction (data not shown).

In sex-specific analyses, shown in the Supplemental Tables 1 and 2, adult BMI was significantly associated with an increased risk of CRC with several methylated IGFBP genes in men but not women, although hazard ratios in women were non-significantly increased. Height was significantly associated with an increased risk of CRC with several methylated IGFBP genes in women, but not men. Other indicators of body size and (non-)occupational physical activity were not clearly associated with CRC risk by IGFBP methylation status. Early life energy restriction was significantly inversely associated with having several methylated IGFBP genes in CRC in men and women. The Supplemental Table 3a-c shows 
Table 2. Characteristics of subcohort members and colorectal cancer (CRC) cases in the Netherlands Cohort Study (19891993)

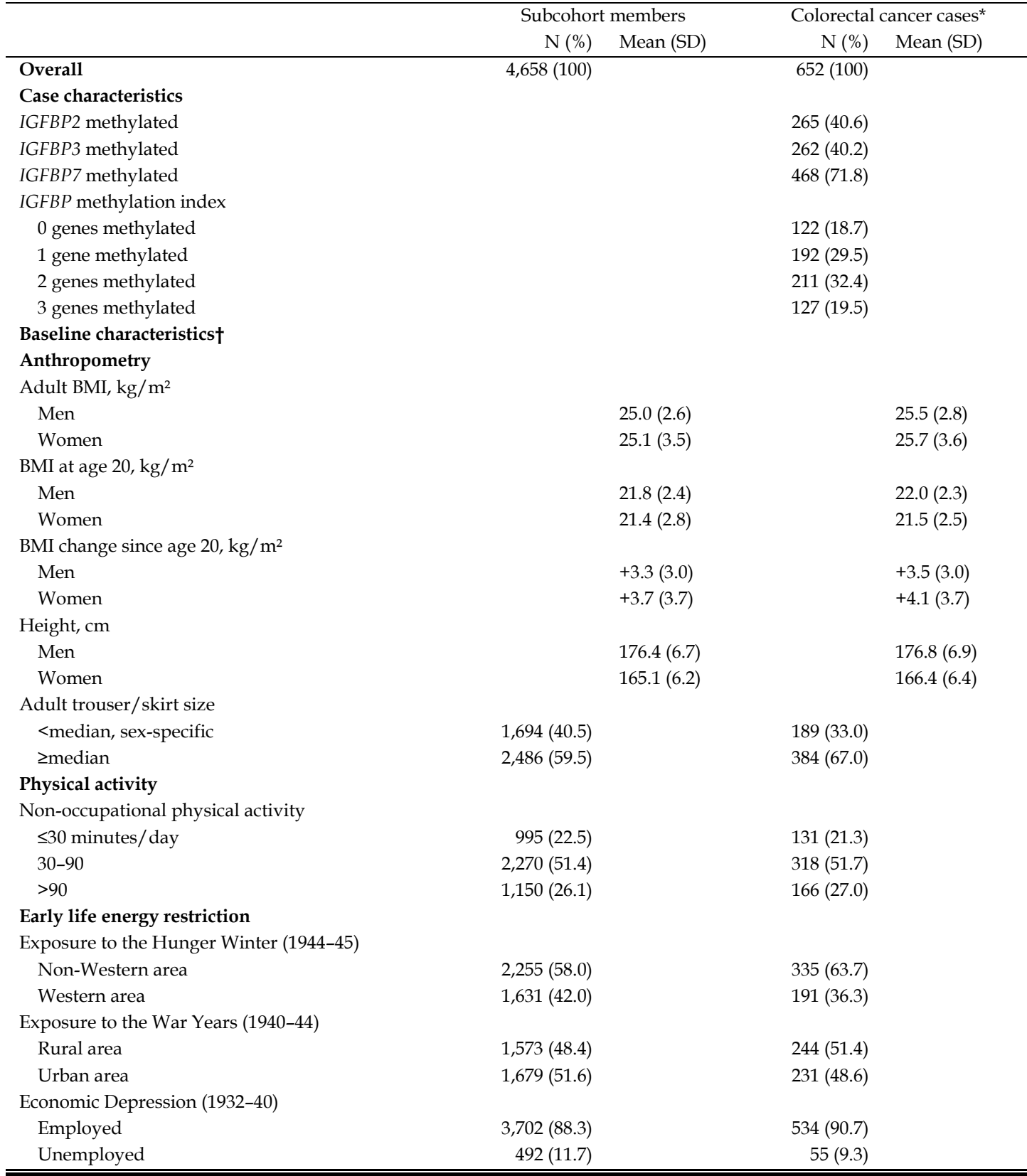

Abbreviations: BMI, body mass index; IGFBP, insulin-like growth factor binding protein; SD, standard deviation

* Cases with successful IGFBP2, IGFBP3, and IGFBP7 methylation analyses.

$\dagger$ Numbers are excluding participants with missing values on adult BMI and non-occupational physical activity. 
Table 3. Molecular and clinical characteristics of colorectal cancer cases by extent of IGFBP methylation in the Netherlands Cohort Study (1989-1993)

\begin{tabular}{|c|c|c|c|c|}
\hline & \multicolumn{4}{|c|}{ IGFBP methylation index } \\
\hline & 0 genes & 1 gene & 2 genes & 3 genes \\
\hline & methylated & methylated & methylated & methylated \\
\hline \multicolumn{5}{|l|}{ Molecular charactistics } \\
\hline \multicolumn{5}{|l|}{ Tumor instability type, N (\%) } \\
\hline MSI (including CIMP) & $6(7.1)$ & $9(5.9)$ & $24(14.4)$ & $26(24.5)$ \\
\hline MSS CIMP & $2(2.4)$ & $19(12.5)$ & $37(22.2)$ & $41(38.7)$ \\
\hline MSS non-CIMP & $76(90.5)$ & $124(81.6)$ & $106(63.5)$ & $39(36.8)$ \\
\hline$P$-value for $\chi^{2}$ test & \multicolumn{4}{|c|}{$<0.001^{*}$} \\
\hline \multicolumn{5}{|l|}{ Clinical characteristics } \\
\hline Age at diagnosis (years), mean (SD) & $67.8(4.5)$ & $67.9(3.9)$ & $67.9(4.5)$ & $68.1(4.1)$ \\
\hline$P$-value for Kruskal-Wallis test & \multicolumn{4}{|c|}{0.98} \\
\hline \multicolumn{5}{|l|}{ Tumor localization, N (\%) } \\
\hline Proximal colon & $29(24.4)$ & $47(25.1)$ & $73(35.3)$ & $64(52.0)$ \\
\hline Distal colon & $43(36.1)$ & $74(39.6)$ & $70(33.8)$ & $21(17.1)$ \\
\hline Rectosigmoid & 15 (12.6) & $25(13.4)$ & $18(8.7)$ & $12(9.8)$ \\
\hline Rectum & $32(26.9)$ & $41(21.9)$ & $46(22.2)$ & $26(21.1)$ \\
\hline$P$-value for $\chi^{2}$ test & \multicolumn{4}{|c|}{$<0.001^{*}$} \\
\hline \multicolumn{5}{|l|}{ TNM stage, N (\%) } \\
\hline Stage 1 & $6(5.3)$ & $14(8.0)$ & $17(8.9)$ & $11(9.2)$ \\
\hline Stage 2 & $30(26.6)$ & $36(20.5)$ & $42(22.0)$ & $19(16.0)$ \\
\hline Stage 3 & $69(61.1)$ & $120(68.2)$ & $119(62.3)$ & $73(61.3)$ \\
\hline Stage 4 & $8(7.1)$ & $6(3.4)$ & $13(6.8)$ & $16(13.5)$ \\
\hline$P$-value for $\chi^{2}$ test & \multicolumn{4}{|c|}{0.08} \\
\hline
\end{tabular}

Abbreviations: CIMP, the CpG island methylator phenotype; IGFBP, insulin-like growth factor binding protein; MSI, microsatellite instability; MSS, microsatellite stable; TNM stage, tumor node metastasis stage

* Remained statistically significant after a Bonferroni correction for multiple testing.

results by IGFBP2, IGFBP3, and IGFBP7 methylation status, respectively, to aid future literature comparison. Overall, these analyses were difficult to interpret as significant associations were not specific to CRCs with methylated IGFBP genes, but were also observed for CRCs with unmethylated IGFBP genes.

\subsection{Discussion}

This is the first study to show that IGFBP methylation may be an important molecular mechanism linking adult BMI and early life energy restriction to CRC risk. In order to fully appreciate our findings, absolute values of BMI should be considered. Using WHO crite- ria as a reference, we report here that $97.2 \%$ of the participants in the lowest tertile in this study fell within the normal adult weight range (BMI $18.5-<25.0 \mathrm{~kg} / \mathrm{m}^{2}$ ), and $80.3 \%$ of the participants in the highest tertile fell within the overweight range (BMI 25.0-<30.0 $\mathrm{kg} / \mathrm{m}^{2}$ ). The significantly increased hazard ratios regarding adult BMI were thus based on a fairly small contrast. Although we must be careful extrapolating findings, higher hazard ratios may be expected in populations with higher obesity prevalences if these allow for a larger BMI contrast to be made. With respect to BMI at age $20,92.3 \%$ of study participants had a BMI $<25.0 \mathrm{~kg} / \mathrm{m}^{2}$, and it may be 


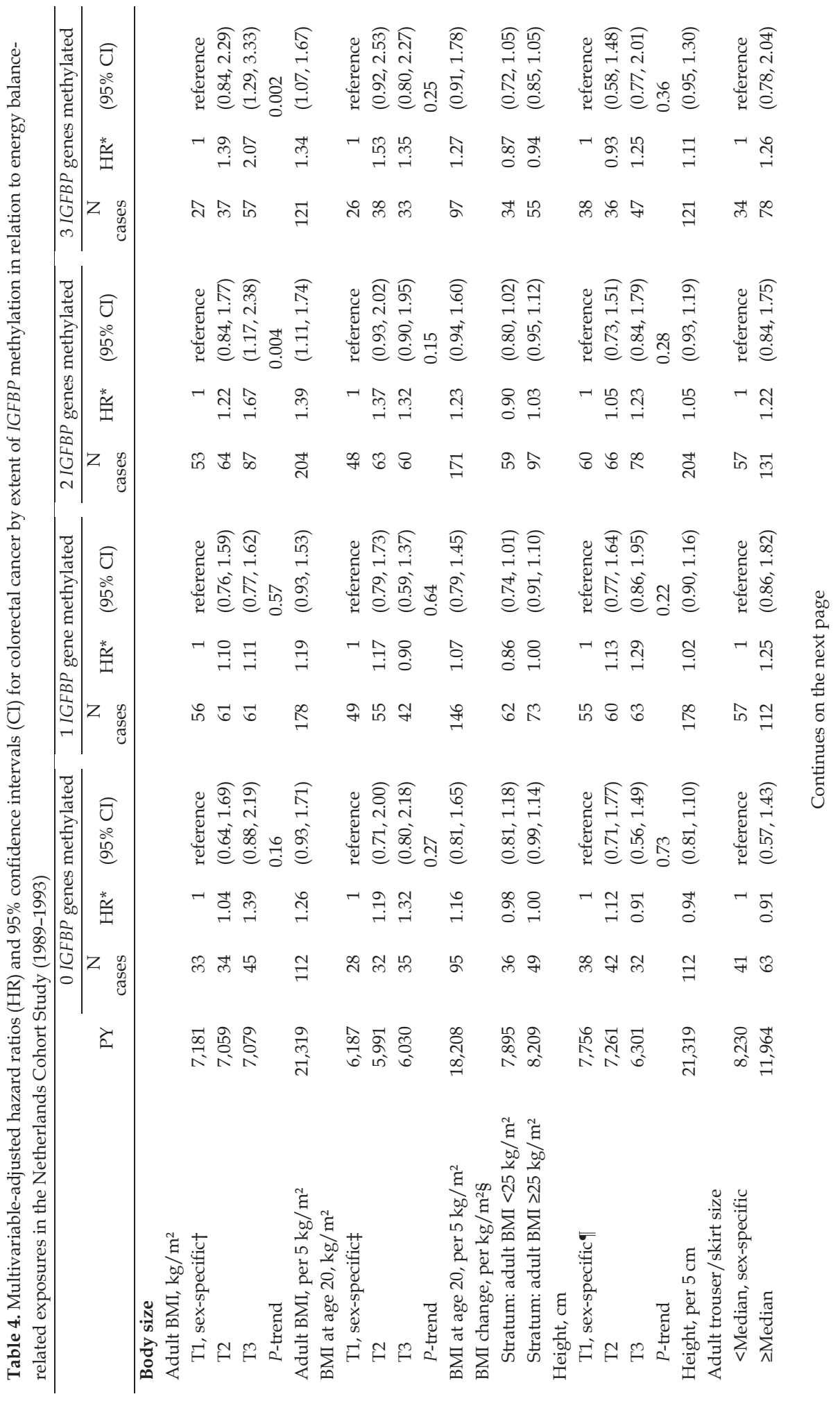




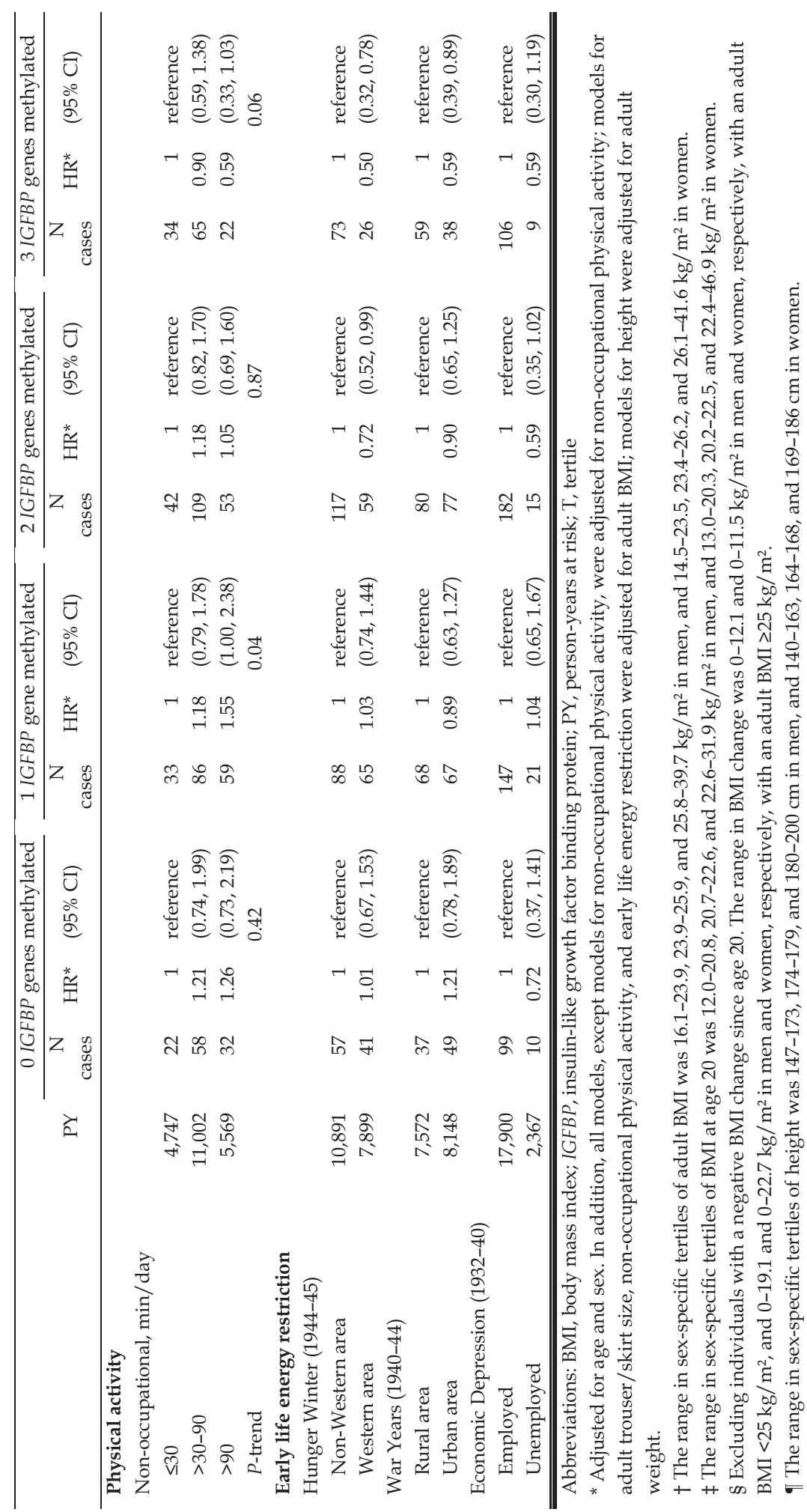


that there was too little contrast between individuals to detect associations.

Validation of our findings is important, as there are no data that link energy balance to CRC through methylation of IGFBP genes. Measures of body size, physical activity, and energy restriction have been shown determinants of CRC risk ${ }^{35}$ and IGFBP blood levels. ${ }^{2}$ Studies have also shown higher IGFBP blood levels to be associated with a decreased CRC risk, many of which focused on IGFBP-3, but results were not always consistent. ${ }^{36-38}$ An explanation for the inconsistent findings may be that the molar ratio between levels of IGF-1 and IGFBPs is more important than absolute levels of these proteins alone. ${ }^{37}$ The amount of bioavailable IGFBPs, furthermore, depends on factors such as IGFBP proteases, ${ }^{3}$ growth hormone, and insulin. ${ }^{36}$ We have measured methylation of IGFBP genes in CRC tumors, and found adult BMI and early life energy restriction associated with CRCs characterized by several methylated genes. The advantage of measuring IGFBP methylation in tumors above IGFBP blood levels may be that it is a local event, likely representative of enduring change. This approach, i.e. the investigation of risk factors in relation to molecular changes in tumors, has been termed molecular pathological epidemiology by Ogino and Stampfer. ${ }^{39}$ Seeing that molecular changes can be viewed as signatures of the tumor's developmental route, molecular pathological epidemiology is a promising tool to gain insights into carcinogenic mechanisms.

It is relatively unclear how IGFBP methylation correlates with CIMP and MSI, which are important phenotypes in CRC associated with methylation of tumor suppressor- and DNA repair genes. Single study observations were a positive correlation between IGFBP7 methylation and CIMP, ${ }^{40}$ and IGFBP3 methylation and CIMP, especially MSS CIMP tumors. ${ }^{12}$ Additionally, IGFBP3 methylation has been used as a CIMP marker. ${ }^{41}$ We observed that the percentage of MSI and MSS CIMP tumors increased with increasing numbers of methylated IGFBP genes, but that still $36.8 \%$ of CRC cases with 3 methylated IGFBP genes had MSS non-CIMP tumors. It may therefore be argued that IGFBP methylation differs from CIMP, which may be reflected in subtle differences in associations with energy balance. Using NLCS data, CIMP in CRC has been associated with early life energy restriction ${ }^{29}$ and BMI at age 20, but not adult BMI;42 and MSI has been associated with height. ${ }^{43}$ Other case-control studies found adult BMI associated with MSS tumors, ${ }^{44,45}$ but not CIMP tumors. ${ }^{46,47}$ In the present study, IGFBP methylation in CRC was associated with adult BMI, particularly in men, and early life energy restriction; sex-specific analyses, furthermore, revealed associations with height in women. Associations were repeatedly in the same direction and as hypothesized, indicating the particular importance of methylation of IGFBP genes in CRC development.

The public health implications of our findings are that weight gain prevention could be especially relevant for specific molecular subgroups. Future research may focus on whether IGFBP methylation can be detected in colorectal polyps and is representative of a defect likely to occur in future lesions. If true, this would open up the exciting possibility of the use of IGFBP methylation, as detected in precursor lesions, as a biomarker for identifying individuals who may in particular benefit from weight gain prevention. The significance of such potential benefit should be investigated in carefully designed intervention studies. So far, methylation has been shown an early event in colorectal tumorigenesis, but the methylation status of multiple adenomas $(\mathrm{n}=$ $78)$ within the same patients $(n=26)$ in a study on CIMP using the methylation markers p16, MINT2, and MINT31 correlated weakly. ${ }^{48}$ However, this study did not include sessile 
serrated adenomas and it may be important to focus on this adenoma type because these lesions often develop through a pathway characterized by methylation, ${ }^{49}$ while methylation might be more random in other lesions.

Major strengths of this study include the prospective design and completeness of followup, making selection and information bias unlikely. A limitation may be that subsitespecific associations could not be studied because of limited power, even though this study is among the largest prospective studies assessing molecular characteristics in CRC. Another limitation may be the single baseline measurement obtained by self-reports. However, our measures cover a considerable period in the lives of study participants, which is important because CRC development is a process of decades, and because, in the case of physical activity, regular, long-term physical activity may predict CRC risk best. ${ }^{32}$ Self- reports of body size have been shown to have good validity. ${ }^{50}$ Regarding early life energy restriction, we used area-exposure data instead of individual data. Individual data may be more accurate, yet a shortcoming of these is the subjective nature. We refrained from subgroup analyses by timing of exposure to energy restriction because of limited power; however, the derived benefit in terms of colorectal cancer risk may have been more pronounced in individuals exposed before or during puberty as compared with those exposed after puberty.

To conclude, IGFBP methylation was shown to link adult BMI and childhood energy restriction to CRC risk. If measurable in early lesions and representative of changes in subsequent lesions, IGFBP methylation might turn out a marker for identifying individuals who may particularly benefit from weight gain prevention. 


\section{References}

1. $\mathrm{Yu}, \mathrm{H}$. \& Rohan, T. Role of the insulin-like growth factor family in cancer development and progression. J Natl Cancer Inst 92, 1472-89 (2000).

2. Kaaks, R. \& Lukanova, A. Energy balance and cancer: the role of insulin and insulin-like growth factor-I. Proc Nutr Soc 60, 91-106 (2001).

3. Durai, R., Yang, W., Gupta, S., Seifalian, A. M. \& Winslet, M. C. The role of the insulin-like growth factor system in colorectal cancer: review of current knowledge. Int J Color Dis 20, 203-20 (2005).

4. Ibanez de Caceres, I. et al. Identification of novel target genes by an epigenetic reactivation screen of renal cancer. Cancer Res 66, 5021-5028 (2006).

5. Sato, H. et al. Growth regulation via insulin-like growth factor binding protein-4 and -2 in association with mutant K-ras in lung epithelia. Am J Pathol 169, 1550-1566 (2006).

6. Lin, J. et al. Methylation patterns of IGFBP7 in colon cancer cell lines are associated with levels of gene expression. J Pathol 212, 83-90 (2007).

7. Ye, F. et al. Decreased expression of insulin-like growth factor binding protein 7 in human colorectal carcinoma is related to DNA methylation. J Cancer Res Clin Oncol 133, 305-314 (2007).

8. Vizioli, M. G. et al. IGFBP7: an oncosuppressor gene in thyroid carcinogenesis. Oncogene 29, 3835-3844 (2010).

9. Chen, Y. et al. IGFBP7 is a p53 target gene inactivated in human lung cancer by DNA hypermethylation. Lung Cancer 73, 38-44 (2011).

10. Van Vlodrop, I. J. H. et al. Analysis of promoter $\mathrm{CpG}$ island hypermethylation in cancer: location, location, location! Clin Cancer Res 17, 4225-4231 (2011).

11. Toyota, M. et al. CpG island methylator phenotype in colorectal cancer. Proc Natl Acad Sci U.S.A. 96, 8681-8686 (1999).

12. Boland, C. R. \& Goel, A. Microsatellite instability in colorectal cancer. Gastroenterology 138, 20732087.e3 (2010).

13. Jass, J. R. Classification of colorectal cancer based on correlation of clinical, morphological and molecular features. Histopathology 50, 113-30 (2007).

14. Van den Brandt, P. A. et al. A large-scale prospective cohort study on diet and cancer in The Netherlands. J Clin Epidemiol 43, 285-295 (1990).

15. Goldbohm, R. A. et al. Validation of a dietary questionnaire used in a large-scale prospective cohort study on diet and cancer. Eur J Clin Nutr 48, 253-65 (1994).

16. Goldbohm, R. A. et al. Reproducibility of a food frequency questionnaire and stability of dietary habits determined from five annually repeated measurements. Eur J Clin Nutr 49, 420-9 (1995).

17. Nevo Table. Dutch food composition table 19867. The Hague, Netherlands: Voorlichtingsbureau voor de Voeding, 1986.

18. Casparie, M. et al. Pathology databanking and biobanking in The Netherlands, a central role for PALGA, the nationwide histopathology and cytopathology data network and archive. Cell Oncol 29, 19-24 (2007).

19. Goldbohm, R. A., van den Brandt, P. A. \& Dorant, E. Estimation of the coverage of Dutch municipalities by cancer registries and PALGA based on hospital discharge data. Tijdschr Soc Gezondheidsz 72, 80-84. (1994).

20. Van den Brandt, P. A., Schouten, L. J., Goldbohm, R. A., Dorant, E. \& Hunen, P. M. Development of a record linkage protocol for use in the Dutch Cancer Registry for Epidemiological Research. Int J Epidemiol 19, 553-558 (1990).

21. WHO: Global Database on Body Mass Index. At http://apps.who.int/bmi/. Accessed: March 10, 2013.

22. Hughes, L. A., Schouten, L. J., Goldbohm, R. A., van den Brandt, P. A. \& Weijenberg, M. P. Selfreported clothing size as a proxy measure for body size. Epidemiology 20, 673-6 (2009).

23. Hettinger, T. H. Ermittlung des Arbeitsenergieumsatzes bei Dynamisch Muskulaerer Arbeit. Dortmund, Germany: Bundesarbeit fuer Arbeitsschutz, 1989.

24. Burger, G., Sandstead, H. \& Drummond, J. Malnutrition and Starvation in Western Netherlands, September 1944 to July 1945. Part I and II. The Hague, the Netherlands: General State Printing Office, 1948

25. Trienekens, G. Tussen ons volk en de honger. De voedselvoorziening, 1940-45. (English translation: Between our nation and the hunger. The food supply, 1940-45). Utrecht, the Netherlands: Stichting Matrijs, 1985.

26. Herman, J. G., Graff, J. R., Myöhänen, S., Nelkin, B. D. \& Baylin, S. B. Methylation-specific PCR: a novel PCR assay for methylation status of CpG islands. Proc. Natl Acad Sci U.S.A. 93, 9821-9826 (1996).

27. Derks, S. et al. Methylation-specific PCR unraveled. Cell Oncol 26, 291-299 (2004). 
28. Weisenberger, D. J. et al. CpG island methylator phenotype underlies sporadic microsatellite instability and is tightly associated with BRAF mutation in colorectal cancer. Nat Genet 38, 787793 (2006).

29. Hughes, L. A. et al. Early life exposure to famine and colorectal cancer risk: a role for epigenetic mechanisms. PloS One 4, e7951 (2009).

30. Suraweera, N. et al. Evaluation of tumor microsatellite instability using five quasimonomorphic mononucleotide repeats and pentaplex PCR. Gastroenterology 123, 1804-1811 (2002).

31. Hughes, L. A. et al. Body size and colorectal cancer risk after 16.3 years of follow-up: an analysis from the Netherlands Cohort Study. Am J Epidemiol 174, 1127-1139 (2011).

32. Simons, C. C. et al. Physical activity, occupational sitting time, and colorectal cancer risk in the Netherlands cohort study. Am J Epidemiol 177, 514-530 (2013).

33. Hughes, L. A. et al. Childhood and adolescent energy restriction and subsequent colorectal cancer risk: results from the Netherlands Cohort Study. Int J Epidemiol 39, 1333-1344 (2010).

34. Barlow, W. E. Robust variance estimation for the case-cohort design. Biometrics 50, 1064-1072 (1994).

35. World Cancer Research Fund / American Institute for Cancer Research. Food, Nutrition, Physical Activity, and the Prevention of Cancer: a Global Perspective. Washington DC: AICR, 2007.

36. Sandhu, M. S., Dunger, D. B. \& Giovannucci, E. L. Insulin, insulin-like growth factor-I (IGF-I), IGF binding proteins, their biologic interactions, and colorectal cancer. J Natl Cancer Inst 94, 972-80 (2002).

37. Ali, O., Cohen, P. \& Lee, K. W. Epidemiology and biology of insulin-like growth factor binding protein-3 (IGFBP-3) as an anti-cancer molecule. Horm Metab Res 35, 726-33 (2003).

38. Renehan, A. G. et al. Insulin-like growth factor (IGF)-I, IGF binding protein-3, and cancer risk: systematic review and meta-regression analysis. Lancet 363, 1346-53 (2004).
39. Ogino, S., Chan, A. T., Fuchs, C. S. \& Giovannucci, E. Molecular pathological epidemiology of colorectal neoplasia: an emerging transdisciplinary and interdisciplinary field. Gut 60, 397-411 (2010).

40. Derks, S. et al. Promoter methylation precedes chromosomal alterations in colorectal cancer development. Cell Oncol 28, 247-257 (2006).

41. Hughes, L. A. et al. The CpG island methylator phenotype in colorectal cancer: progress and problems. Biochim Biophys Acta 1825, 77-85 (2012).

42. Hughes, L. A. et al. Body size, physical activity and risk of colorectal cancer with or without the CpG island methylator phenotype (CIMP). PloS One 6, e18571 (2011).

43. Hughes, L. A. et al. Body size and risk for colorectal cancers showing BRAF mutations or microsatellite instability: a pooled analysis. Int J Epidemiol 41, 1060-1072 (2012).

44. Slattery, M. L. et al. Associations between cigarette smoking, lifestyle factors, and microsatellite instability in colon tumors. J Natl Cancer Inst 92, 1831-1836 (2000).

45. Campbell, P. T. et al. Case-control study of overweight, obesity, and colorectal cancer risk, overall and by tumor microsatellite instability status. J Natl Cancer Inst 102, 391-400 (2010).

46. Slattery, M. L. et al. Diet and lifestyle factor associations with $\mathrm{CpG}$ island methylator phenotype and BRAF mutations in colon cancer. Int J Cancer 120, 656-663 (2007).

47. Slattery, M. L. et al. Diet, physical activity, and body size associations with rectal tumor mutations and epigenetic changes. Cancer Causes Control 21, 1237-1245 (2010).

48. Rashid, A., Shen, L., Morris, J. S., Issa, J. P. \& Hamilton, S. R. CpG island methylation in colorectal adenomas. Am J Pathol 159, 1129-1135 (2001).

49. Jass, J. R. Molecular heterogeneity of colorectal cancer: Implications for cancer control. Surg Oncol 16 Suppl 1, S7-9 (2007).

50. Spencer, E. A., Appleby, P. N., Davey, G. K. \& Key, T. J. Validity of self-reported height and weight in 4808 EPIC-Oxford participants. Public Heal Nutr 5, 561-5 (2002). 


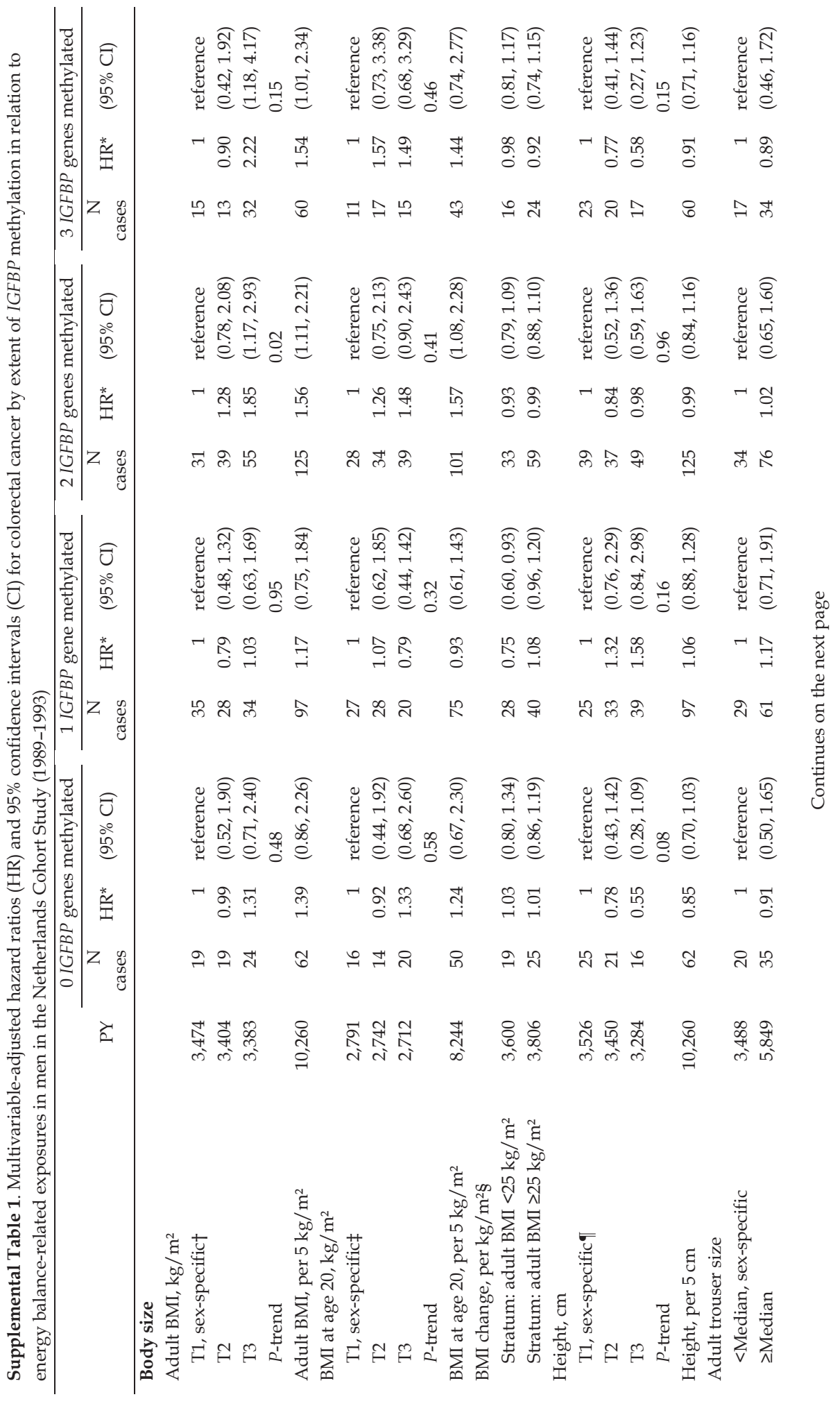




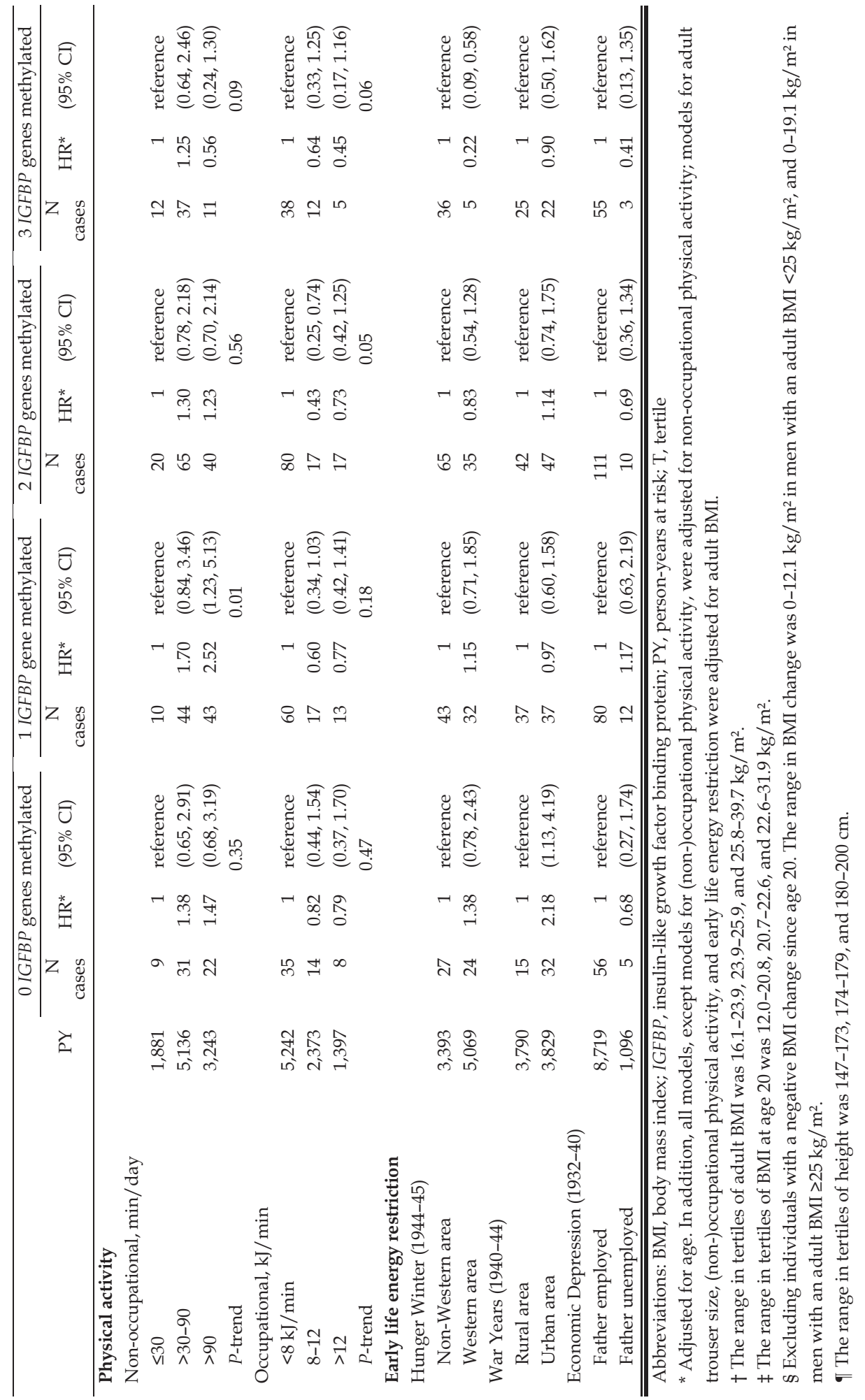




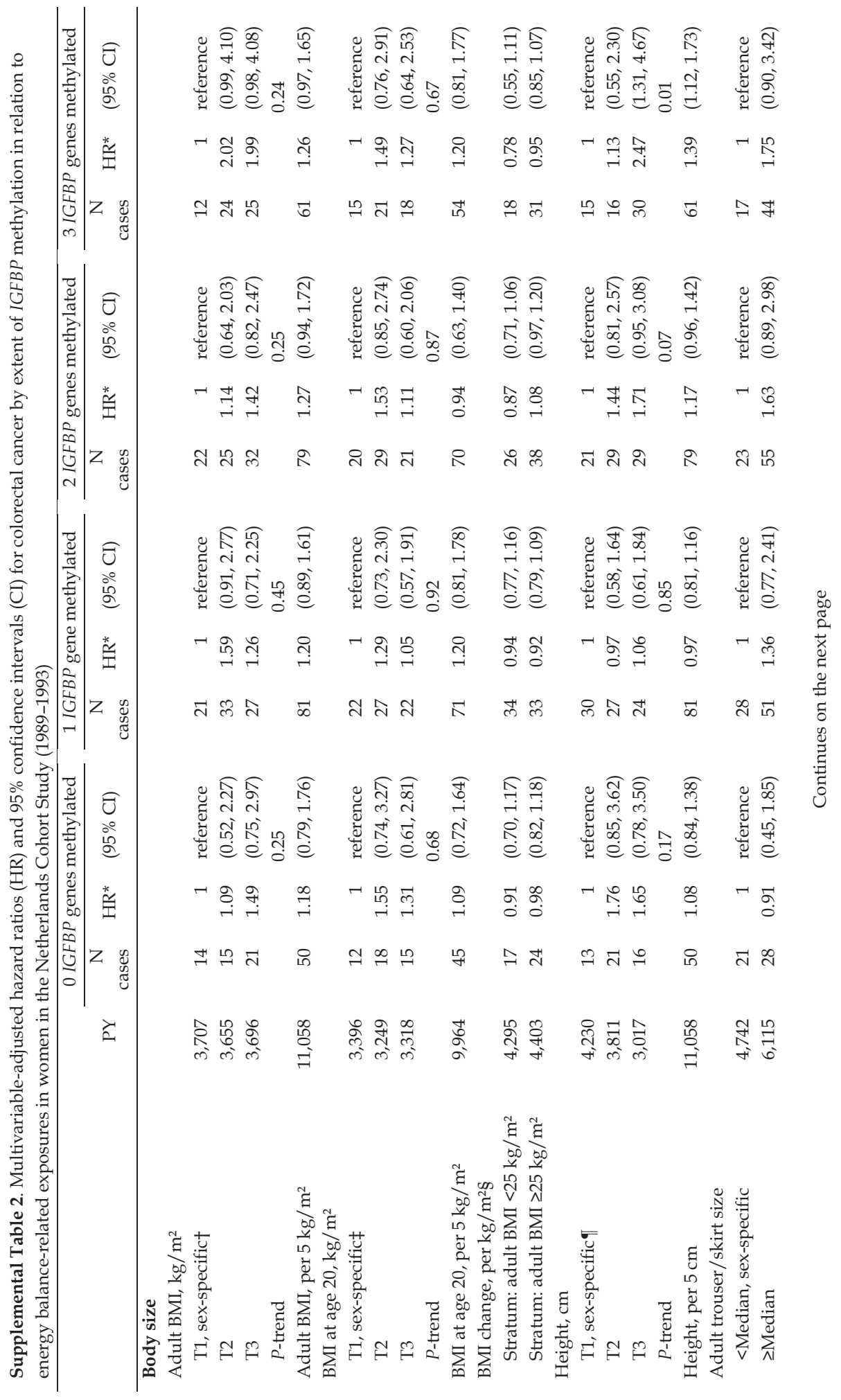




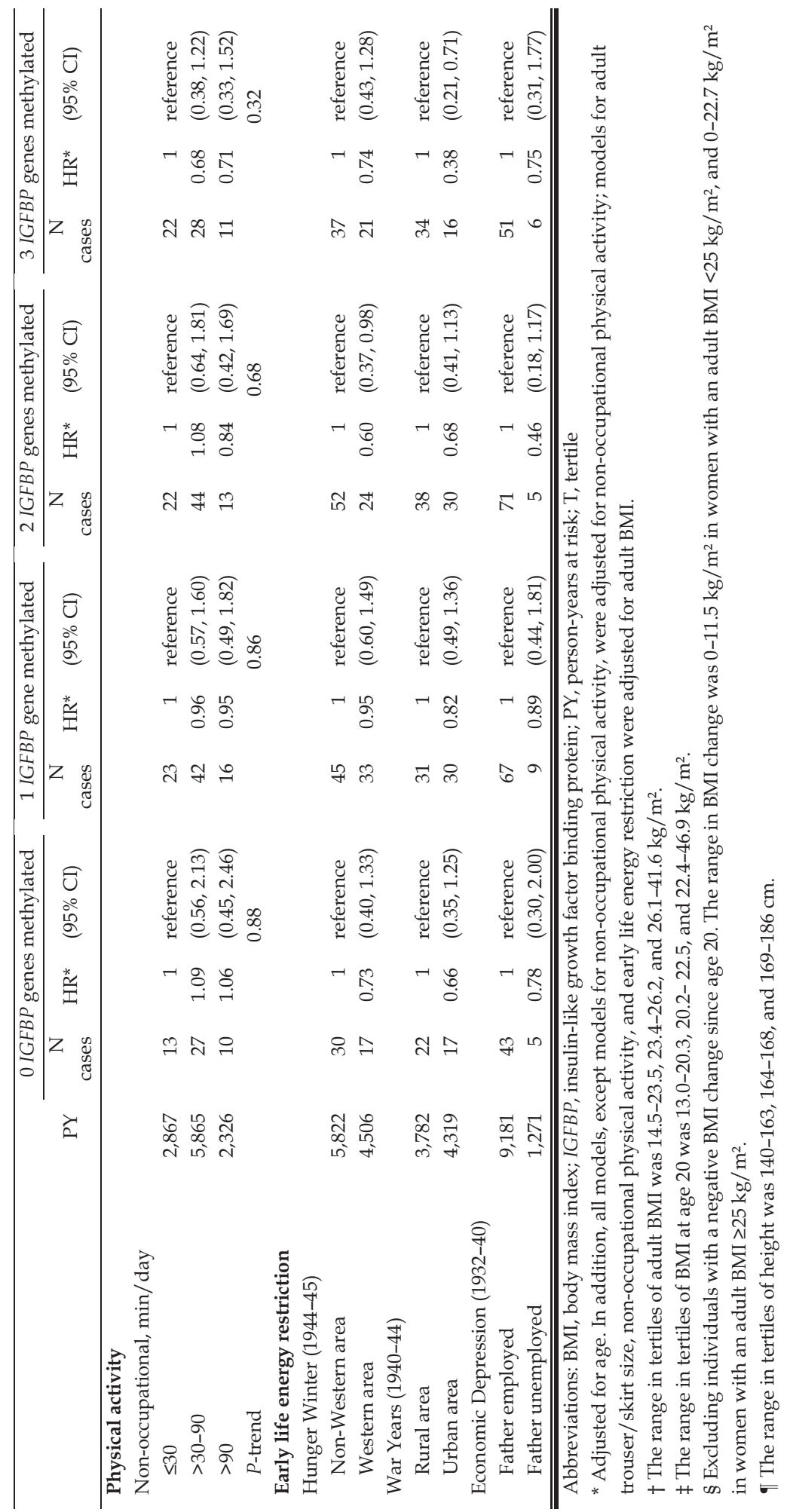


Supplemental Table 3a. Multivariable-adjusted hazard ratios (HR) and 95\% confidence intervals (CI) for colorectal cancer by IGFBP2, IGFBP3, and IGFBP7 methylation status in relation to energy balance-related exposures in the Netherlands Cohort Study

\begin{tabular}{|c|c|c|c|c|c|c|c|}
\hline & \multirow[b]{2}{*}{ PY } & \multicolumn{3}{|c|}{ IGFBP2 methylated } & \multicolumn{3}{|c|}{ IGFBP2 unmethylated } \\
\hline & & $\begin{array}{r}\mathrm{N} \\
\text { cases }\end{array}$ & $\mathrm{HR}^{*}$ & $(95 \% \mathrm{CI})$ & $\begin{array}{r}\mathrm{N} \\
\text { cases }\end{array}$ & $\mathrm{HR}^{*}$ & $(95 \% \mathrm{CI})$ \\
\hline \multicolumn{8}{|l|}{ Body size } \\
\hline \multicolumn{8}{|l|}{ Adult BMI, $\mathrm{kg} / \mathrm{m}^{2}$} \\
\hline $\mathrm{T} 1$, sex-specific $\dagger$ & 7,181 & 69 & 1 & reference & 110 & 1 & reference \\
\hline $\mathrm{T} 2$ & 7,059 & 86 & 1.26 & $(0.91,1.75)$ & 133 & 1.22 & $(0.94,1.59)$ \\
\hline $\mathrm{T} 3$ & 7,079 & 113 & 1.64 & $(1.20,2.24)$ & 150 & 1.38 & $(1.06,1.78)$ \\
\hline$P$-trend & & & & 0.002 & & & 0.02 \\
\hline Adult BMI, per $5 \mathrm{~kg} / \mathrm{m}^{2}$ & 21,319 & 268 & 1.25 & $(1.05,1.50)$ & 393 & 1.29 & $(1.09,1.53)$ \\
\hline \multicolumn{8}{|l|}{ BMI at age $20, \mathrm{~kg} / \mathrm{m}^{2}$} \\
\hline T1, sex-specific $\ddagger$ & 6,187 & 62 & 1 & reference & 102 & 1 & reference \\
\hline $\mathrm{T} 2$ & 5,991 & 93 & 1.57 & $(1.13,2.19)$ & 107 & 1.10 & $(0.83,1.46)$ \\
\hline $\mathrm{T} 3$ & 6,029 & 65 & 1.11 & $(0.78,1.59)$ & 119 & 1.23 & $(0.93,1.62)$ \\
\hline$P$-trend & & & & 0.53 & & & 0.14 \\
\hline BMI at age 20 , per $5 \mathrm{~kg} / \mathrm{m}^{2}$ & 18,208 & 220 & 1.13 & $(0.90,1.42)$ & 328 & 1.20 & $(0.98,1.47)$ \\
\hline \multicolumn{8}{|l|}{ BMI change, per $\mathrm{kg} / \mathrm{m}^{2} \S$} \\
\hline Stratum: adult BMI $<25 \mathrm{~kg} / \mathrm{m}^{2}$ & 7,895 & 82 & 0.88 & $(0.79,0.98)$ & 127 & 0.93 & $(0.84,1.03)$ \\
\hline Stratum: adult BMI $\geq 25 \mathrm{~kg} / \mathrm{m}^{2}$ & 8,209 & 119 & 0.99 & $(0.93,1.06)$ & 173 & 1.00 & $(0.93,1.07)$ \\
\hline \multicolumn{8}{|l|}{ Height, $\mathrm{cm}$} \\
\hline T1, sex-specific & 7,756 & 83 & 1 & reference & 127 & 1 & reference \\
\hline $\mathrm{T} 2$ & 7,261 & 86 & 1.03 & $(0.76,1.41)$ & 128 & 1.01 & $(0.77,1.31)$ \\
\hline $\mathrm{T} 3$ & 6,301 & 99 & 1.25 & $(0.91,1.72)$ & 138 & 1.14 & $(0.86,1.51)$ \\
\hline$P$-trend & & & & 0.18 & & & 0.36 \\
\hline Height, per $5 \mathrm{~cm}$ & 21,319 & 268 & 1.08 & $(0.97,1.20)$ & 393 & 1.01 & $(0.92,1.10)$ \\
\hline \multicolumn{8}{|l|}{ Adult trouser/skirt size } \\
\hline$<$ Median, sex-specific & 8,230 & 86 & 1 & reference & 119 & 1 & reference \\
\hline$\geq$ Median & 11,964 & 160 & 1.02 & $(0.75,1.39)$ & 252 & 1.26 & $(0.96,1.65)$ \\
\hline \multicolumn{8}{|l|}{ Physical activity } \\
\hline \multicolumn{8}{|l|}{ Non-occupational, min/day } \\
\hline$\leq 30$ & 4,747 & 64 & 1 & reference & 84 & 1 & reference \\
\hline$>30-90$ & 11,002 & 143 & 1.02 & $(0.75,1.40)$ & 196 & 1.07 & $(0.82,1.40)$ \\
\hline$>90$ & 5,569 & 61 & 0.82 & $(0.57,1.19)$ & 113 & 1.16 & $(0.86,1.56)$ \\
\hline$P$-trend & & & & 0.28 & & & 0.32 \\
\hline \multicolumn{8}{|l|}{ Early life energy restriction } \\
\hline \multicolumn{8}{|l|}{ Hunger Winter (1944-45) } \\
\hline Non-Western area & 10,891 & 157 & 1 & reference & 201 & 1 & reference \\
\hline Western area & 7,899 & 70 & 0.63 & $(0.47,0.84)$ & 137 & 0.96 & $(0.76,1.21)$ \\
\hline \multicolumn{8}{|l|}{ War Years (1940-44) } \\
\hline Rural area & 7,572 & 125 & 1 & reference & 140 & 1 & reference \\
\hline Urban area & 8,148 & 88 & 0.65 & $(0.49,0.86)$ & 159 & 1.04 & $(0.82,1.33)$ \\
\hline \multicolumn{8}{|l|}{ Economic Depression (1932-40) } \\
\hline Father employed & 17,900 & 236 & 1 & reference & 336 & 1 & reference \\
\hline Father unemployed & 2,367 & 20 & 0.61 & $(0.38,0.97)$ & 41 & 0.88 & $(0.62,1.23)$ \\
\hline
\end{tabular}

Abbreviations: BMI, body mass index; IGFBP, insulin-like growth factor binding protein; PY, person-years at risk; T, tertile

* Adjusted for age and sex. In addition, all models, except models for non-occupational physical activity, were adjusted for nonoccupational physical activity; models for adult trouser/skirt size, non-occupational physical activity, and early life energy restriction were adjusted for adult BMI; models for height were adjusted for adult weight. $†$ The range in sex-specific tertiles of adult BMI was 16.1-23.9, 23.9-25.9, and $25.8-39.7 \mathrm{~kg} / \mathrm{m}^{2}$ in men, and $14.5-23.5,23.4-26.2$, and $26.1-41.6 \mathrm{~kg} / \mathrm{m}^{2}$ in women. $¥$ The range in sex-specific tertiles of BMI at age 20 was $12.0-20.8,20.7-22.6$, and $22.6-31.9 \mathrm{~kg} / \mathrm{m}^{2}$ in men, and $13.0-20.3,20.2-22.5$, and $22.4-46.9 \mathrm{~kg} / \mathrm{m}^{2}$ in women. § Excluding individuals with a negative BMI change since age 20. The range in BMI change was $0-12.1$ and $0-11.5 \mathrm{~kg} / \mathrm{m}^{2}$ in men and women, respectively, with an adult BMI $<25 \mathrm{~kg} / \mathrm{m}^{2}$, and $0-19.1$ and $0-22.7 \mathrm{~kg} / \mathrm{m}^{2}$ in men and women, respectively, with an adult BMI $\geq 25 \mathrm{~kg} / \mathrm{m}^{2}$. ๆ The range in sexspecific tertiles of height was 147-173, 174-179, and 180-200 cm in men, and 140-163, 164-168, and 169-186 cm in women. 
Supplemental Table 3b. Multivariable-adjusted hazard ratios (HR) and 95\% confidence intervals (CI) for colorectal cancer by IGFBP2, IGFBP3, and IGFBP7 methylation status in relation to energy balance-related exposures in the Netherlands Cohort Study

\begin{tabular}{|c|c|c|c|c|c|c|c|}
\hline & \multirow[b]{2}{*}{ PY } & \multicolumn{3}{|c|}{ IGFBP3 methylated } & \multicolumn{3}{|c|}{ IGFBP3 unmethylated } \\
\hline & & $\begin{array}{r}\mathrm{N} \\
\text { cases }\end{array}$ & $\mathrm{HR}^{*}$ & $(95 \% \mathrm{CI})$ & $\begin{array}{r}\mathrm{N} \\
\text { cases }\end{array}$ & $\mathrm{HR}^{*}$ & $(95 \% \mathrm{CI})$ \\
\hline \multicolumn{8}{|l|}{ Body size } \\
\hline \multicolumn{8}{|l|}{ Adult BMI, $\mathrm{kg} / \mathrm{m}^{2}$} \\
\hline $\mathrm{T} 1$, sex-specific $\dagger$ & 7,181 & 63 & 1 & reference & 110 & 1 & reference \\
\hline $\mathrm{T} 2$ & 7,059 & 82 & 1.32 & $(0.94,1.85)$ & 116 & 1.06 & $(0.81,1.40)$ \\
\hline $\mathrm{T} 3$ & 7,079 & 109 & 1.73 & $(1.25,2.39)$ & 149 & 1.38 & $(1.06,1.79)$ \\
\hline$P$-trend & & & & 0.001 & & & 0.02 \\
\hline Adult BMI, per $5 \mathrm{~kg} / \mathrm{m}^{2}$ & 21,319 & 254 & 1.28 & $(1.06,1.55)$ & 375 & 1.31 & $(1.11,1.55)$ \\
\hline \multicolumn{8}{|l|}{ BMI at age $20, \mathrm{~kg} / \mathrm{m}^{2}$} \\
\hline T1, sex-specific $\ddagger$ & 6,187 & 65 & 1 & reference & 91 & 1 & reference \\
\hline $\mathrm{T} 2$ & 5,991 & 71 & 1.14 & $(0.81,1.62)$ & 122 & 1.40 & $(1.05,1.86)$ \\
\hline $\mathrm{T} 3$ & 6,029 & 76 & 1.25 & $(0.89,1.75)$ & 98 & 1.13 & $(0.84,1.53)$ \\
\hline$P$-trend & & & & 0.20 & & & 0.39 \\
\hline BMI at age 20 , per $5 \mathrm{~kg} / \mathrm{m}^{2}$ & 18,208 & 212 & 1.20 & $(0.93,1.54)$ & 311 & 1.14 & $(0.94,1.40)$ \\
\hline \multicolumn{8}{|l|}{ BMI change, per $\mathrm{kg} / \mathrm{m}^{2} \S$} \\
\hline Stratum: adult BMI $<25 \mathrm{~kg} / \mathrm{m}^{2}$ & 7,895 & 77 & 0.93 & $(0.83,1.05)$ & 117 & 0.88 & $(0.78,0.98)$ \\
\hline Stratum: adult BMI $\geq 25 \mathrm{~kg} / \mathrm{m}^{2}$ & 8,209 & 115 & 0.97 & $(0.89,1.05)$ & 169 & 1.02 & $(0.96,1.09)$ \\
\hline \multicolumn{8}{|l|}{ Height, $\mathrm{cm}$} \\
\hline $\mathrm{T} 1$, sex-specific & 7,756 & 76 & 1 & reference & 121 & 1 & reference \\
\hline $\mathrm{T} 2$ & 7,261 & 75 & 0.97 & $(0.70,1.36)$ & 132 & 1.08 & $(0.83,1.41)$ \\
\hline $\mathrm{T} 3$ & 6,301 & 103 & 1.38 & $(0.98,1.95)$ & 122 & 1.05 & $(0.79,1.38)$ \\
\hline$P$-trend & & & & 0.06 & & & 0.74 \\
\hline Height, per $5 \mathrm{~cm}$ & 21,319 & 254 & 1.12 & $(1.00,1.26)$ & 375 & 0.97 & $(0.89,1.06)$ \\
\hline \multicolumn{8}{|l|}{ Adult trouser/skirt size } \\
\hline$<$ Median, sex-specific & 8,230 & 70 & 1 & reference & 123 & 1 & reference \\
\hline$\geq$ Median & 11,964 & 166 & 1.42 & $(1.01,2.00)$ & 227 & 1.02 & $(0.79,1.32)$ \\
\hline \multicolumn{8}{|l|}{ Physical activity } \\
\hline \multicolumn{8}{|l|}{ Non-occupational, min/day } \\
\hline$\leq 30$ & 4,747 & 61 & 1 & reference & 74 & 1 & reference \\
\hline$>30-90$ & 11,002 & 138 & 1.05 & $(0.77,1.43)$ & 188 & 1.16 & $(0.87,1.54)$ \\
\hline$>90$ & 5,569 & 55 & 0.79 & $(0.54,1.15)$ & 113 & 1.31 & $(0.96,1.78)$ \\
\hline$P$-trend & & & & 0.19 & & & 0.09 \\
\hline \multicolumn{8}{|l|}{ Early life energy restriction } \\
\hline \multicolumn{8}{|l|}{ Hunger Winter (1944-45) } \\
\hline Non-Western area & 10,891 & 150 & 1 & reference & 194 & 1 & reference \\
\hline Western area & 7,899 & 64 & 0.60 & $(0.44,0.81)$ & 130 & 0.95 & $(0.75,1.20)$ \\
\hline \multicolumn{8}{|l|}{ War Years (1940-44) } \\
\hline Rural area & 7,572 & 110 & 1 & reference & 144 & 1 & reference \\
\hline Urban area & 8,148 & 83 & 0.69 & $(0.51,0.93)$ & 150 & 0.96 & $(0.75,1.22)$ \\
\hline \multicolumn{8}{|l|}{ Economic Depression (1932-40) } \\
\hline Father employed & 17,900 & 225 & 1 & reference & 321 & 1 & reference \\
\hline Father unemployed & 2,367 & 19 & 0.60 & $(0.37,0.97)$ & 38 & 0.86 & $(0.60,1.22)$ \\
\hline
\end{tabular}

Abbreviations: BMI, body mass index; IGFBP, insulin-like growth factor binding protein; PY, person-years at risk; T, tertile

* Adjusted for age and sex. In addition, all models, except models for non-occupational physical activity, were adjusted for nonoccupational physical activity; models for adult trouser/skirt size, non-occupational physical activity, and early life energy restriction were adjusted for adult BMI; models for height were adjusted for adult weight. $†$ The range in sex-specific tertiles of adult BMI was 16.1-23.9, 23.9-25.9, and $25.8-39.7 \mathrm{~kg} / \mathrm{m}^{2}$ in men, and $14.5-23.5,23.4-26.2$, and $26.1-41.6 \mathrm{~kg} / \mathrm{m}^{2}$ in women. $¥$ The range in sex-specific tertiles of BMI at age 20 was $12.0-20.8,20.7-22.6$, and $22.6-31.9 \mathrm{~kg} / \mathrm{m}^{2}$ in men, and $13.0-20.3,20.2-22.5$, and $22.4-46.9 \mathrm{~kg} / \mathrm{m}^{2}$ in women. § Excluding individuals with a negative BMI change since age 20. The range in BMI change was $0-12.1$ and $0-11.5 \mathrm{~kg} / \mathrm{m}^{2}$ in men and women, respectively, with an adult BMI $<25 \mathrm{~kg} / \mathrm{m}^{2}$, and $0-19.1$ and $0-22.7 \mathrm{~kg} / \mathrm{m}^{2}$ in men and women, respectively, with an adult BMI $\geq 25 \mathrm{~kg} / \mathrm{m}^{2}$. ๆ The range in sexspecific tertiles of height was 147-173, 174-179, and 180-200 cm in men, and 140-163, 164-168, and 169-186 cm in women. 
Supplemental Table 3c. Multivariable-adjusted hazard ratios (HR) and 95\% confidence intervals (CI) for colorectal cancer by IGFBP2, IGFBP3, and IGFBP7 methylation status in relation to energy balance-related exposures in the Netherlands Cohort Study

\begin{tabular}{|c|c|c|c|c|c|c|c|}
\hline & \multirow[b]{2}{*}{ PY } & \multicolumn{3}{|c|}{ IGFBP7 methylated } & \multicolumn{3}{|c|}{ IGFBP7 unmethylated } \\
\hline & & $\begin{array}{r}\mathrm{N} \\
\text { cases }\end{array}$ & $\mathrm{HR}^{*}$ & $(95 \% \mathrm{CI})$ & $\begin{array}{r}\mathrm{N} \\
\text { cases }\end{array}$ & $\mathrm{HR}^{*}$ & $(95 \% \mathrm{CI})$ \\
\hline \multicolumn{8}{|l|}{ Body size } \\
\hline \multicolumn{8}{|l|}{ Adult BMI, $\mathrm{kg} / \mathrm{m}^{2}$} \\
\hline $\mathrm{T} 1$, sex-specific $\dagger$ & 7,181 & 123 & 1 & reference & 55 & 1 & reference \\
\hline $\mathrm{T} 2$ & 7,059 & 149 & 1.22 & $(0.95,1.57)$ & 70 & 1.28 & $(0.89,1.85)$ \\
\hline $\mathrm{T} 3$ & 7,079 & 195 & 1.59 & $(1.25,2.03)$ & 64 & 1.19 & $(0.82,1.71)$ \\
\hline$P$-trend & & & & $<0.001$ & & & 0.36 \\
\hline Adult BMI, per $5 \mathrm{~kg} / \mathrm{m}^{2}$ & 21,319 & 467 & 1.37 & $(1.18,1.58)$ & 189 & 1.09 & $(0.85,1.39)$ \\
\hline \multicolumn{8}{|l|}{ BMI at age $20, \mathrm{~kg} / \mathrm{m}^{2}$} \\
\hline T1, sex-specific $\ddagger$ & 6,187 & 113 & 1 & reference & 50 & 1 & reference \\
\hline $\mathrm{T} 2$ & 5,991 & 146 & 1.35 & $(1.04,1.75)$ & 53 & 1.11 & $(0.74,1.65)$ \\
\hline $\mathrm{T} 3$ & 6,029 & 124 & 1.16 & $(0.89,1.51)$ & 57 & 1.21 & $(0.82,1.79)$ \\
\hline$P$-trend & & & & 0.27 & & & 0.33 \\
\hline BMI at age 20 , per $5 \mathrm{~kg} / \mathrm{m}^{2}$ & 18,208 & 383 & 1.19 & $(0.99,1.43)$ & 160 & 1.12 & $(0.85,1.48)$ \\
\hline \multicolumn{8}{|l|}{ BMI change, per $\mathrm{kg} / \mathrm{m}^{2} \S$} \\
\hline Stratum: adult BMI $<25 \mathrm{~kg} / \mathrm{m}^{2}$ & 7,895 & 144 & 0.88 & $(0.80,0.97)$ & 65 & 0.95 & $(0.83,1.09)$ \\
\hline Stratum: adult BMI $\geq 25 \mathrm{~kg} / \mathrm{m}^{2}$ & 8,209 & 213 & 1.00 & $(0.95,1.06)$ & 76 & 0.96 & $(0.87,1.06)$ \\
\hline \multicolumn{8}{|l|}{ Height, $\mathrm{cm}$} \\
\hline $\mathrm{T} 1$, sex-specific & 7,756 & 144 & 1 & reference & 64 & 1 & reference \\
\hline $\mathrm{T} 2$ & 7,261 & 147 & 0.99 & $(0.78,1.27)$ & 67 & 1.11 & $(0.78,1.58)$ \\
\hline $\mathrm{T} 3$ & 6,301 & 176 & 1.21 & $(0.94,1.57)$ & 58 & 1.08 & $(0.73,1.58)$ \\
\hline$P$-trend & & & & 0.14 & & & 0.69 \\
\hline Height, per $5 \mathrm{~cm}$ & 21,319 & 467 & 1.04 & $(0.96,1.13)$ & 189 & 1.01 & $(0.90,1.14)$ \\
\hline \multicolumn{8}{|l|}{ Adult trouser/skirt size } \\
\hline$<$ Median, sex-specific & 8,230 & 128 & 1 & reference & 76 & 1 & reference \\
\hline$\geq$ Median & 11,964 & 306 & 1.32 & $(1.03,1.70)$ & 101 & 0.84 & $(0.59,1.20)$ \\
\hline \multicolumn{8}{|l|}{ Physical activity } \\
\hline \multicolumn{8}{|l|}{ Non-occupational, min/day } \\
\hline$\leq 30$ & 4,747 & 108 & 1 & reference & 36 & 1 & reference \\
\hline$>30-90$ & 11,002 & 237 & 1.01 & $(0.79,1.28)$ & 101 & 1.28 & $(0.87,1.89)$ \\
\hline$>90$ & 5,569 & 122 & 0.97 & $(0.74,1.29)$ & 52 & 1.25 & $(0.81,1.92)$ \\
\hline$P$-trend & & & & 0.85 & & & 0.33 \\
\hline \multicolumn{8}{|l|}{ Early life energy restriction } \\
\hline \multicolumn{8}{|l|}{ Hunger Winter (1944-45) } \\
\hline Non-Western area & 10,891 & 251 & 1 & reference & 103 & 1 & reference \\
\hline Western area & 7,899 & 143 & 0.81 & $(0.65,1.01)$ & 64 & 0.87 & $(0.63,1.20)$ \\
\hline \multicolumn{8}{|l|}{ War Years (1940-44) } \\
\hline Rural area & 7,572 & 194 & 1 & reference & 69 & 1 & reference \\
\hline Urban area & 8,148 & 176 & 0.84 & $(0.67,1.05)$ & 71 & 0.93 & $(0.66,1.31)$ \\
\hline \multicolumn{8}{|l|}{ Economic Depression (1932-40) } \\
\hline Father employed & 17,900 & 400 & 1 & reference & 166 & 1 & reference \\
\hline Father unemployed & 2,367 & 44 & 0.79 & $(0.57,1.09)$ & 18 & 0.78 & $(0.47,1.29)$ \\
\hline
\end{tabular}

Abbreviations: BMI, body mass index; IGFBP, insulin-like growth factor binding protein; PY, person-years at risk; T, tertile

* Adjusted for age and sex. In addition, all models, except models for non-occupational physical activity, were adjusted for nonoccupational physical activity; models for adult trouser/skirt size, non-occupational physical activity, and early life energy restriction were adjusted for adult BMI; models for height were adjusted for adult weight. † The range in sex-specific tertiles of adult BMI was 16.1-23.9, 23.9-25.9, and $25.8-39.7 \mathrm{~kg} / \mathrm{m}^{2}$ in men, and $14.5-23.5,23.4-26.2$, and $26.1-41.6 \mathrm{~kg} / \mathrm{m}^{2}$ in women. ¥ The range in sex-specific tertiles of BMI at age 20 was $12.0-20.8,20.7-22.6$, and $22.6-31.9 \mathrm{~kg} / \mathrm{m}^{2}$ in men and $13.0-20.3,20.2-22.5$, and $22.4-46.9 \mathrm{~kg} / \mathrm{m}^{2}$ in women. $\S$ Excluding individuals with a negative BMI change since age 20. The range in BMI change was $0-12.1$ and $0-11.5 \mathrm{~kg} / \mathrm{m}^{2}$ in men and women, respectively, with an adult $\mathrm{BMI}<25 \mathrm{~kg} / \mathrm{m}^{2}$, and $0-19.1$ and $0-22.7 \mathrm{~kg} / \mathrm{m}^{2}$ in men and women, respectively, with an adult BMI $\geq 25 \mathrm{~kg} / \mathrm{m}^{2}$. $\uparrow$ The range in sexspecific tertiles of height was 147-173, 174-179, and 180-200 cm in men, and 140-163, 164-168, and 169-186 cm in women. 


\section{Body size, physical activity and risk of colorectal cancer with or without the $\mathrm{CpG}$ island methylator phenotype (CIMP)}

Laura AE Hughes, Colinda CJM Simons, Piet A van den Brandt, R Alexandra Goldbohm, Anton FPM de Goeij, Adriaan P de Bruïne, Manon van Engeland, Matty P Weijenberg

PLoS One. 2011 Apr 5;6(4):e18571. 


\section{-Abstract}

Background: We investigated how body size and physical activity influence the risk of the CpG island methylator phenotype (CIMP) in colorectal cancer (CRC).

Methods: In the Netherlands Cohort Study $(n=120,852)$, risk factors were self-reported at baseline in 1986. After 7.3 years of follow-up, 603 cases and 4,631 subcohort members were available. CIMP status according to the Weisenberger markers was determined using methylationspecific PCR on DNA from paraffin-embedded tumor tissue. Hazard rate ratios (HR) and 95\% confidence intervals for CIMP (27.7\%) and non-CIMP (72.3\%) tumors were calculated according to BMI, BMI at age 20, BMI change, trouser/skirt size, height, and physical activity.

Results: BMI modeled per $5 \mathrm{~kg} / \mathrm{m}^{2}$ increase was associated with both CIMP and non-CIMP tumors, however, hazard ratios were attenuated when additionally adjusted for trouser/skirt size. Trouser/skirt size, per 2 size increase, was associated with both tumor subtypes, even after adjustment for BMI (CIMP, HR = 1.20, 95\% CI: 1.01, 1.43; non-CIMP, HR = 1.14, 95\% CI: 1.04, 1.28). Height per $5 \mathrm{~cm}$ was associated with both tumor subtypes, but hazard ratios were attenuated when adjusted for body weight. BMI at age 20 was positively associated with an increased risk of CIMP tumors and the association was significantly less pronounced for non-CIMP tumors $(P$-heterogeneity $=0.01)$. Physical activity was inversely associated with both subtypes, but a dose-response association was observed only for non-CIMP tumors $(P$-trend $=0.02)$.

Conclusion: Body size, especially central adiposity, may increase the risk of both CIMP and non-CIMP tumors. Body fat at young age may differentially influence risk. Physical activity appears to decrease the risk of CRC regardless of these molecular subtypes. 


\subsection{Introduction}

It is well-accepted that indicators of energy balance influence the risk of colorectal cancer (CRC). A high body mass index (BMI), waist circumference, and adult attained height are clear risk factors for CRC, while physical activity has been shown to be protective. ${ }^{1}$ Although CRC is one of the best described cancers in terms of genetic and epigenetic events involved, ${ }^{2-5}$ little is known about how measures of anthropometry and physical activity are associated with different molecular subsets of this disease. Elucidating potential differences in risk between molecular subtypes of CRC may lead to a better understanding of CRC, treatment, and prevention. This is especially important as the global prevalence of overweight and obesity continues to rise.

A distinct characteristic of epigenetic instability in CRC is the CpG island methylator phenotype (CIMP), characterized by numerous promoter $\mathrm{CpG}$ island hypermethylated tumor suppressor- and DNA repair genes. ${ }^{6-9}$ This in turn is associated with transcriptional silencing of gene expression. ${ }^{10}$ Few studies have investigated associations between indicators of energy balance and CIMP status, and those that have, only considered BMI as an indicator of body size. In a case-control setting, Slattery et al. reported an association between a high BMI and CIMP low but not CIMP high colon tumors, ${ }^{11}$ and no association between BMI and CIMP status in rectal tumors. ${ }^{12}$ Vigorous physical activity was associated with both CIMP high and CIMP low colon tumors, but not rectal tumors. ${ }^{11,12}$ It has been hypothesized that DNA methylation is a consequence of inflammation. ${ }^{13,14}$ Central adiposity is also associated with chronic inflammation. ${ }^{15}$ Therefore, considering waist circumference as a risk factor for CIMP in addition to BMI is important. Additionally, methylation is thought to be an early event in CRC, ${ }^{16}$ so considering height and BMI at a young age may also be informa- tive as these variables are indicative of in utero and early life exposures. ${ }^{17}$

Using the prospective Netherlands Cohort Study on diet and cancer (NLCS), we investigated the association between BMI, clothing size (as a proxy for waist circumference) and physical activity and the risk of developing a tumor characterized by CIMP. Furthermore, in attempt to elucidate if timing of exposure is important for modulating this risk, we also investigated associations according to BMI at age 20, BMI change, and adult-attained height.

\subsection{Methods}

\subsubsection{Study population and design}

The NLCS is a prospective cohort study that was initiated in 1986 to investigate the association between diet and the development of cancer. It includes 58,279 men and 62,573 women between the ages of 55-69 years at baseline who completed a self-administered food frequency questionnaire involving 150 food items as well as questions on dietary habits, lifestyle, health, and demographics. Municipal registries from throughout the Netherlands were used to constitute an efficient sampling frame. The NLCS uses a casecohort approach for data processing and analysis; case subjects were derived from the entire cohort, and the number of person-years at risk for the entire cohort was estimated from a subcohort of 5,000 men and women who were randomly sampled from the full cohort at baseline. All subcohort members who reported prevalent cancer (excluding skin cancer) at baseline were excluded from analyses, leaving 4,654 . Further details of the NLCS design have been described. ${ }^{18-20}$

Incident CRC cases were identified by annual record linkage to 9 regional cancer registries and a national pathology database (PALGA). ${ }^{21}$ 
The completeness of cancer follow-up is almost $100 \% .{ }^{22}$ Paraffin-embedded tumor material from CRC patients was retrieved, as described previously. ${ }^{23}$ In total, 734 incident CRC patients were identified from a followup period of 7.3 years after baseline, excluding the first two years of follow-up, of whom a PALGA report of the lesion as well as sufficient DNA was available. ${ }^{23}$

The study protocol was approved by the Medical Ethics Committees of the University Hospital Maastricht and TNO Nutrition. On recruitment, participants were informed in writing of the details of the study and its objectives. In accordance with the regulations at that time, written informed consent was obtained when participants returned the completed baseline questionnaire. Tumor material was collected after approval by the ethical review boards of Maastricht University, the National Cancer Registry, and PALGA.

\subsubsection{Ascertainment of risk factors}

Anthropometric variables. Height (cm), body weight $(\mathrm{kg})$, and body weight at age $20(\mathrm{~kg})$ were self-reported on the baseline questionnaire. From these variables, BMI and BMI at age 20, and BMI change were subsequently calculated. At baseline, individuals were also asked to report their lower body (trouser or skirt) clothing size from their clothing label (Dutch sizes). Trouser/skirt size has been shown to be an adequate proxy measure for waist circumference when predicting cancer risk in the NLCS, and details of how clothing size corresponds to waist measurements in men and women in this Dutch population have been published. ${ }^{24} \mathrm{BMI}, \mathrm{BMI}$ at age 20, trouser/skirt size, and height were categorized into approximate sex-specific quartiles. As in previous NLCS analyses, BMI change was categorized as: $<0,0-4,4-8$, and $>8$ $\mathrm{kg} / \mathrm{m}^{2} .^{25}$
Physical activity. With respect to physical activity and CRC risk in the NLCS population, occupational physical activity appears to be more important for men and recreational physical activity for women for predicting risk. ${ }^{26}$ Therefore, we used these two variables to create sex-specific categories of 'low,' 'intermediate' and 'high' physical activity.

Occupational physical activity was derived from data on participants' occupational history. Using information on the type of job and the duration, energy expenditure was calculated for the longest held job. Energy expenditure was based on a rating system developed by Hettinger et al. ${ }^{27}$ and distinguishes between work of low, moderate and high activity which corresponds to an energy expenditure of $<8,8-12$, and $>12 \mathrm{~kJ} / \mathrm{min}$. Men were categorized into the 'low' category if their occupational physical activity was $<8 \mathrm{~kJ} / \mathrm{min}$, 'intermediate' if they fell into the $8-12 \mathrm{~kJ} / \mathrm{min}$ category, and 'high' if their occupational physical activity was $>12 \mathrm{~kJ} / \mathrm{min}$.

Baseline non-occupational physical activity was calculated based on two questions. The first (open-ended) question was 'How many minutes do you spend on average per day walking or cycling to your work, to go shopping or to take out your dog?' The reported time spent per day was categorized into $\leq 10$, $>10-30,>30-60$, and $>60 \mathrm{~min} /$ day. The second question was 'How many hours of your leisure time do you spend on average per week on 1) recreational cycling, walking, 2) gardening, doing odd jobs, and 3) sports, gymnastics?' Answering possibilities were never, $<1$, $1-2$, and $\geq 2$ hour(s)/week. The time spent on these activities and the minutes spent per day on walking or cycling to work, to go shopping or to take out the dog were summed to obtain an overall measure of baseline nonoccupational physical activity, with categories $\leq 30,>30-60,>60-90$, and >90 $\mathrm{min} /$ day. 'Low' physical activity was defined as $\leq 30 \mathrm{~min} /$ day, 
'intermediate' as >30-90 min/day, and 'high' as $>90 \mathrm{~min} /$ day.

\subsubsection{Promoter methylation analyses}

CIMP in tumor tissue of CRC cases was defined by $\mathrm{CpG}$ island promoter hypermethylation of at least 3 out of 5 methylation markers (CACNA1G, IGF2, NEUROG1, RUNX3, and SOCS1), as proposed by Weisenberger et al. ${ }^{9}$ The methylation status of these markers was determined by bisulfite modification of $500 \mathrm{ng}$ genomic DNA using a commercially available kit (Zymo Research), and subsequent methylation-specific PCR (MSP). ${ }^{28,29}$ We chose to use MSP as a method because it is effective, specific and does not require specific equipment. It has been shown that results from MSP are in accordance with other technologies, such as MethyLight. ${ }^{30}$ Additionally, the methylation status of two other genes, APC and MLH1, were determined and we added them to the CIMP markers to create a methylation index of seven genes.

To facilitate MSP analysis on DNA retrieved from formalin-fixed, paraffin-embedded tissue, DNA was first amplified with flanking PCR primers that amplify bisulfite-modified DNA but do not preferentially amplify methylated or unmethylated DNA. The resulting fragment was used as a template for the MSP reaction. All PCRs were carried out with controls for unmethylated alleles (DNA from normal lymphocytes), methylated alleles [normal lymphocyte DNA treated in vitro with SssI methyltransferase (New England Biolabs, Ipswich, MA)], and a control without DNA. Ten microliters of each MSP reaction was directly loaded on to nondenaturing 6\% polyacrylamide gels stained with ethidium bromide and visualised under UV illumination. The MSP analyses were successful for $81 \%, 79 \%, 79 \%, 90 \%, 83 \%, 93 \%$, and $93 \%$ out of the 734 cases for CACNA1G, IGF2, NEU-
ROG1, RUNX3, SOCS1, MLH1, and APC respectively.

\subsubsection{Statistical analyses}

Data were analyzed with Stata (version 10, Statacorp, College Station, TX, USA). Cox proportional hazards analysis using the casecohort approach was used to obtain hazard ratios and 95\% confidence intervals for the association between measures of anthropometry and physical activity and CRC characterized by CIMP status. To improve statistical power, we considered men and women together.

Tests for effect modification by sex were not statistically significant. The proportional hazards assumption was tested using the scaled Schoenfeld residuals and by visual inspection of the -log-log transformed hazard curves. To account for the additional variance introduced by sampling the subcohort from the entire cohort, standard errors were estimated using the robust option. Statistical significance was tested at the 0.05 level.

For all anthropometric variables in question, three models were considered. The first was adjusted only for age and sex. The second was additionally adjusted for variables identified as being associated with both CRC and energy balance from previous literature. These included family history of CRC (yes/no), smoking status (never smoker, ex-smoker, current smoker), socioeconomic status (level of education: primary school, junior high school, senior high school, higher vocational school, or university), total energy intake (kcal/day), alcohol intake (0, 0.1-4, 5-14, 15-29, $\geq 30$ g/day), physical activity (low, medium, high as previously described), and consumption of red meat, fruit, vegetables, and grains (g/day). Finally, models were mutually adjusted for other anthropometric variables. Models including BMI and BMI at age 20 were mutually adjusted for trouser/skirt size; the model including BMI change was adjusted for 
BMI at age 20; the model including trouser/skirt size was adjusted for BMI; and the model including height was adjusted for body weight.

We modeled physical activity adjusted for age and sex, and additionally adjusted for trouser/skirt size, family history of CRC (yes/no), smoking status (never, ex-, current smoker), socioeconomic status (level of educational: primary school, junior high school, senior high school, higher vocational school, or university), total energy intake (kcal/day), alcohol intake (0, 0.1-4, 5-14, 15-29, $\geq 30 \mathrm{~g} /$ day), clothing size, and consumption of red meat, fruit, vegetables, and fiber (g/day).

To assess how measures of anthropometry and physical activity were associated with the extent of promoter methylation in CRC tumors, we used the aforementioned methylation index to categorize cases into one of three groups: '0-1 genes methylated', '2-3 genes methylated', or '4-7 genes methylated'. Of the 734 cases, 556 had sufficient information to be classified into one of the three categories. Models including anthropometric variables were adjusted for age, sex, and mutually adjusted for other anthropometric variables as previously described, and the model for physical activity was adjusted for age and sex.

Tests for heterogeneity were done to evaluate differences between subtypes of tumors (CIMP versus non-CIMP) using the competing risks procedure in Stata. However, the standard error for the difference of the log-hazard ratios from this procedure assumes independence of both estimated hazard ratios, which would overestimate that standard error and thus overestimate the $P$-values for their difference. Therefore, these $P$-values and the associated confidence intervals were estimated based on a bootstrapping method that was developed for the case-cohort design, as de- scribed previously. ${ }^{31}$ Each bootstrap analysis was based on 1,000 replications.

\subsection{Results}

Baseline and molecular characteristics are described in Table 1. Proportionally, CIMP CRC cases were more likely to be female, have a tumor in the proximal colon, and be older than non-CIMP cases.

Associations between measures of anthropometry and CRC risk according to CIMP status are shown in Table 2. After adjustment for age and sex, BMI modeled per $5 \mathrm{~kg} / \mathrm{m}^{2}$ increase was statistically significantly associated with CIMP tumors [hazard ratio $(\mathrm{HR})=1.29$, $95 \%$ confidence interval (CI): 1.01, 1.66], and a statistically significant dose-response relationship was observed when modelling quartiles of BMI ( $P$-trend = 0.02). However, when models were mutually adjusted for trouser/skirt size, these associations were greatly attenuated. BMI was also positively, though not statistically significantly, associated with nonCIMP tumors. These associations were also attenuated when mutually adjusted for trouser/skirt size. The hazard ratios for CIMP and non-CIMP tumors did not differ significantly from one another $(P$-heterogeneity $=0.78)$. $\mathrm{BMI}$ at age 20 modeled per $5 \mathrm{~kg} / \mathrm{m}^{2}$ increase was associated with an increased risk of both subtypes, but hazard ratios did not reach statistical significance. When BMI at age 20 was modeled in quartiles, a statistically significant trend was observed for CIMP tumors $(P=$ 0.03 ). This trend became borderline significant when the model was adjusted for trouser/skirt size $(P=0.07)$. The test for heterogeneity between tumor subtypes was statistically significant with respect to BMI at age 20, even after adjustment for trouser/skirt size $(P$-heterogeneity $=0.01)$. No statistically significant observations were observed with respect to BMI change. 


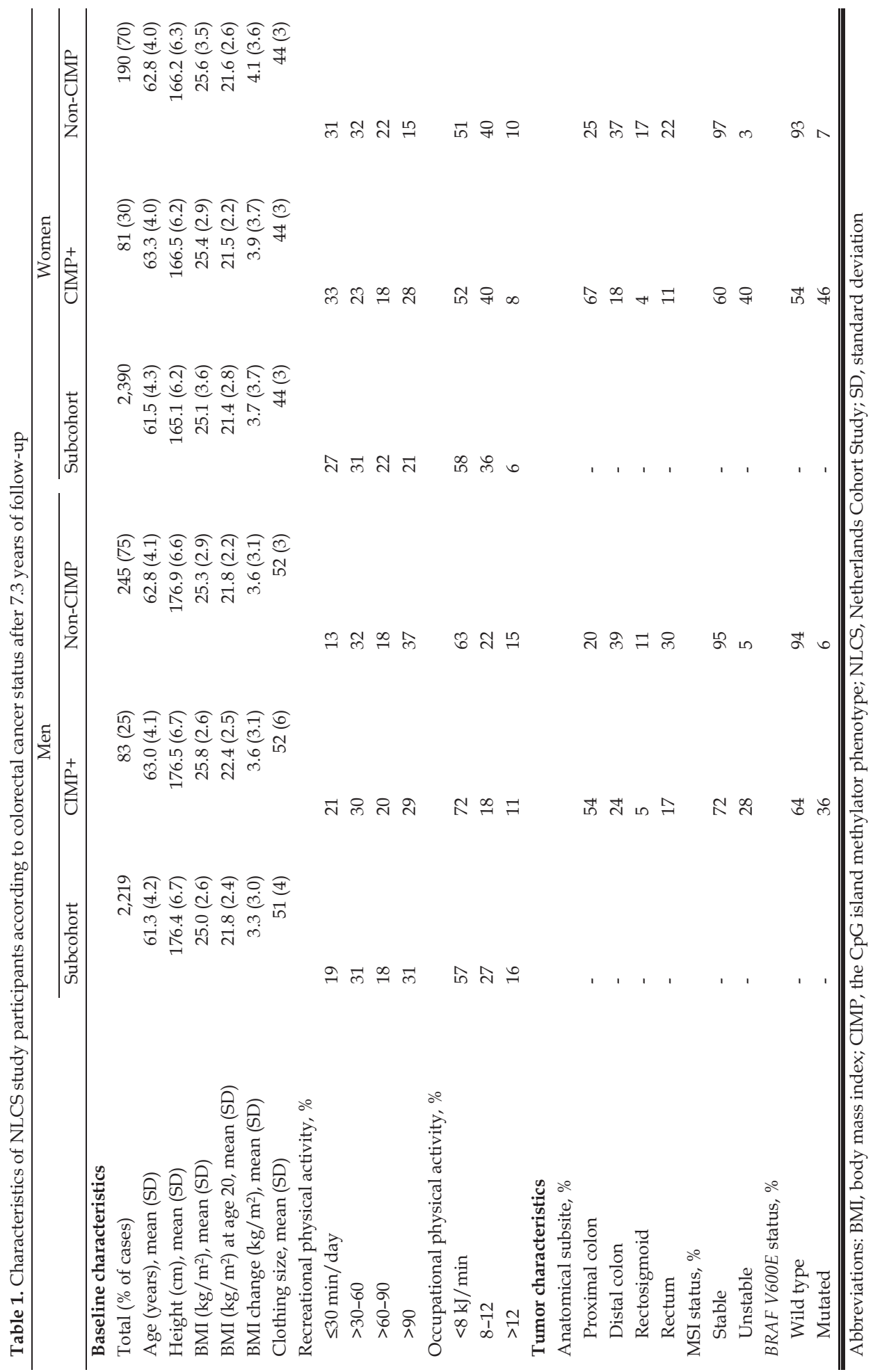




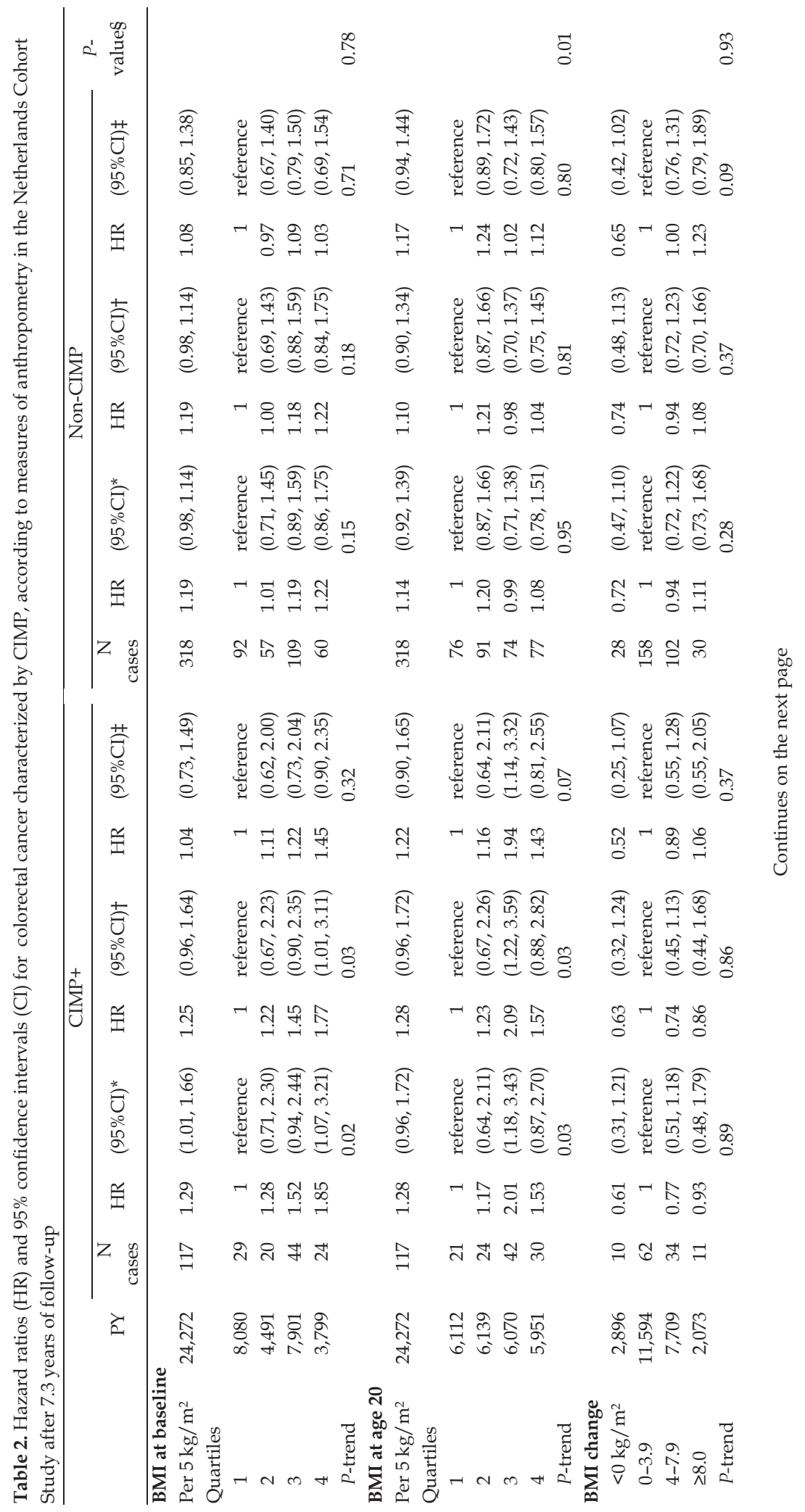




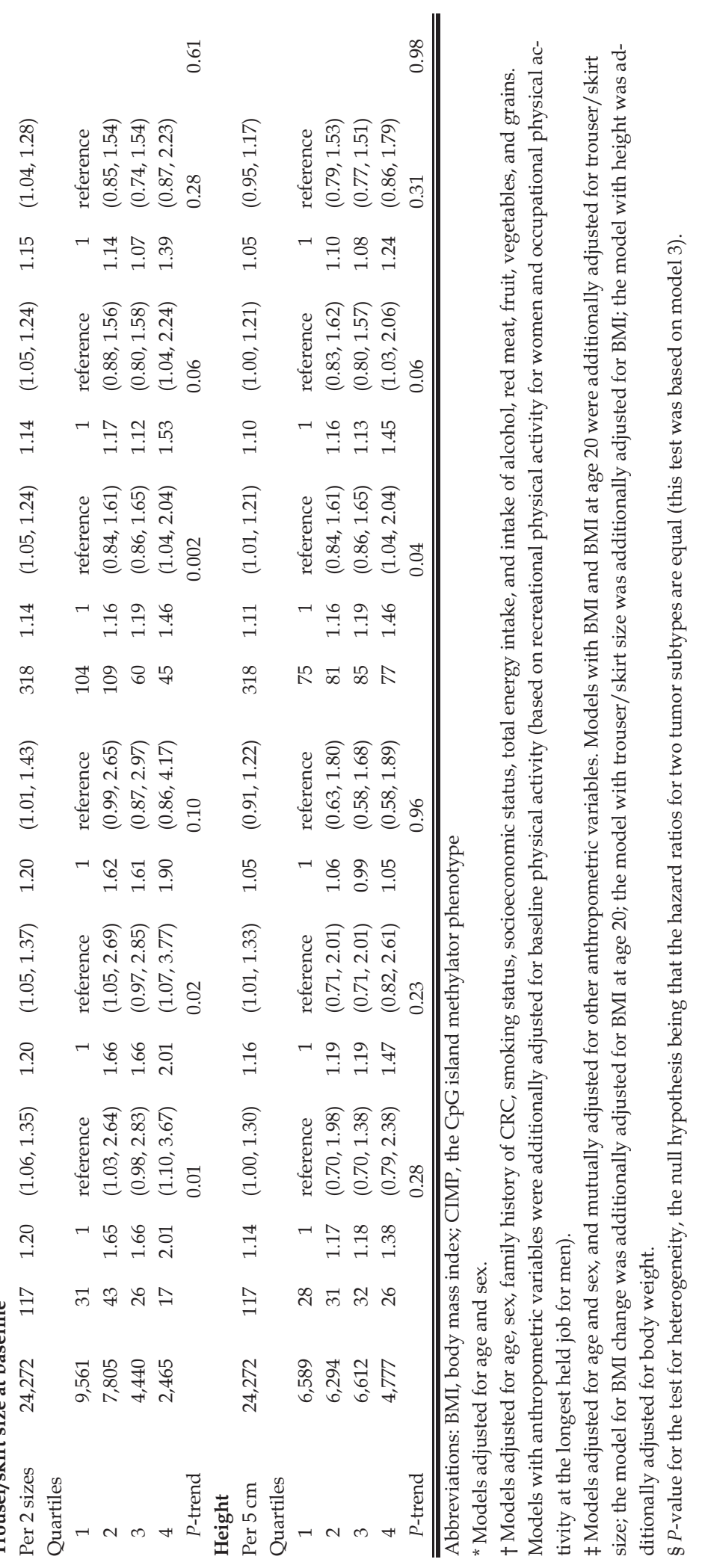


Trouser/skirt size, modeled per 2 unit size increase, was associated with both CIMP (HR $=1.20,95 \%$ CI: $1.05,1.37)$ and non-CIMP tumors (HR $=1.14,95 \%$ CI: $1.05,1.24)$ after multivariate adjustment, and these associations remained even when models were mutually adjusted for BMI. When trouser/skirt size was considered in approximate quartiles, the test for trend was significant for CIMP $(P=0.02)$ and borderline significant for non-CIMP $(P=$ $0.06)$ tumors, although these were attenuated when models were mutually adjusted for BMI. Associations observed for CIMP and non-CIMP tumors were not statistically significantly different from each other $(P$ heterogeneity $=0.61$ ).

Height, modeled per $5 \mathrm{~cm}$ increase was associated with both CIMP ( $\mathrm{HR}=1.16,95 \% \mathrm{CI}$ : $1.01,1.33)$ and non-CIMP (HR $=1.10,95 \% \mathrm{CI}$ : $1.00,1.21)$ tumors after multivariate adjustment. A borderline statistically significant trend was observed for the risk of non-CIMP tumors when height was considered in quartiles (highest versus lowest quartile $\mathrm{HR}=1.45$, 95\% CI: 1.03, 2.06; $P$-trend $=0.06)(P$ heterogeneity $=0.98)$. These associations were attenuated when the model was mutually adjusted for body weight.

With low physical activity as the reference category, there was no inverse dose-response association between physical activity and CIMP tumors, although the hazard ratio for intermediate physical activity was statistically significant (HR $=0.50,95 \%$ CI: 0.30, 0.82) (Table 3). An inverse association was observed in a dose-response fashion for non-CIMP tumors (intermediate versus low physical activity HR $=0.80,95 \%$ CI: 0.61, 1.04; high versus low physical activity HR $=0.67,95 \%$ CI: $0.47,0.96$; $P$-trend $=0.02$ ); however, associations with CIMP and non-CIMP tumors did not differ significantly from each other $(P$-heterogeneity $=0.33$ ).
Associations for a tumor methylation index in relation to anthropometric risk factors and physical activity are shown in Table 4 . There was no clear pattern with respect to the degree of methylation, however, when modeled per 2 unit size increase, trouser/skirt size was associated with tumors displaying the highest level of methylation (4-7 genes methylated $\mathrm{HR}=1.29$, 95\% CI: 1.06, 1.58).

\subsection{Discussion}

These data suggest that adult body fatness and height may increase the risk of CRC, but are not differentially associated with CIMP and non-CIMP tumors. Contrarily, BMI at age 20 may be a stronger risk factor for CIMP+ tumors. Baseline physical activity appears to decrease the risk of CRC regardless of CIMP status.

A major strength of this study is that we investigated associations in a prospective cohort setting. The NLCS has almost complete ascertainment of CRC cases and very little loss to follow-up. Although the number of total CRC cases after 7.3 years of follow-up in the NLCS was substantial, the number of cases with the CIMP phenotype was small. With limited power to detect associations, it is possible that some findings arose by chance. Another potential limitation of this study is that anthropometric variables were obtained by selfreport. However, there are many examples in the literature showing that this method is a valid and reliable tool for assessing body weight and height in cohort studies. ${ }^{32-35}$

To our knowledge, associations between indicators of energy balance and CIMP status of colorectal tumors have been reported only by Slattery et al. in a case-control setting. ${ }^{11,12}$ In addition to the study design, there are differences between our studies which should be taken into account when comparing results. 


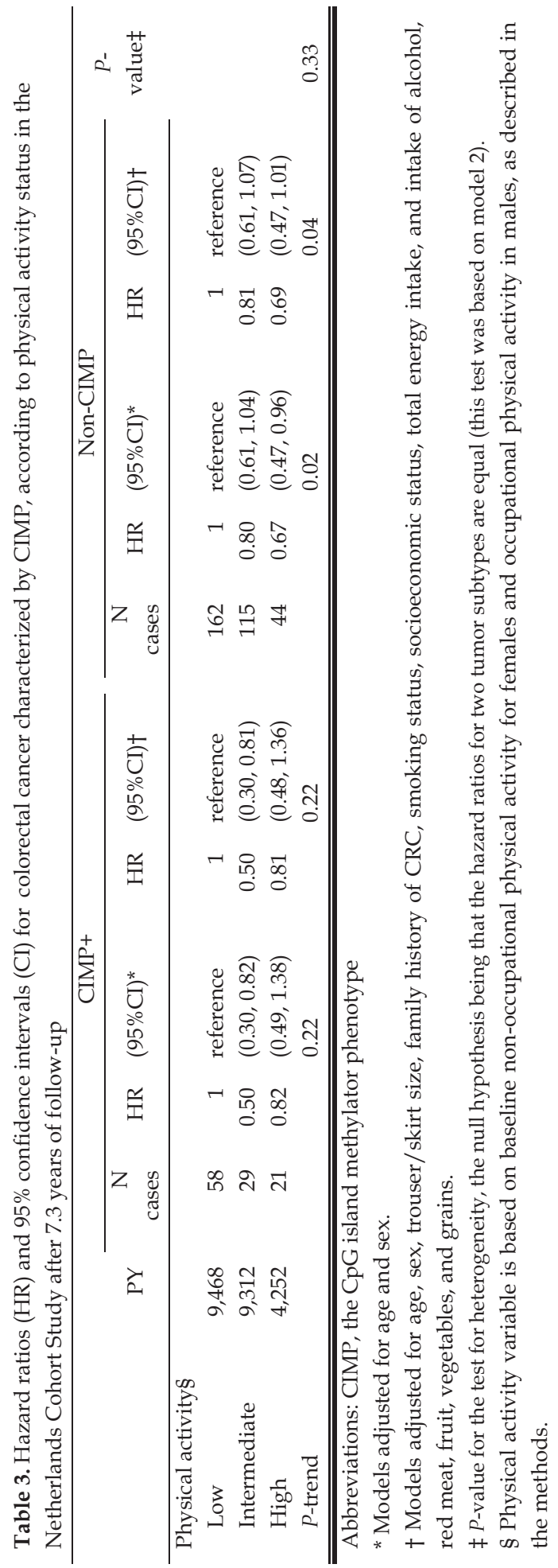




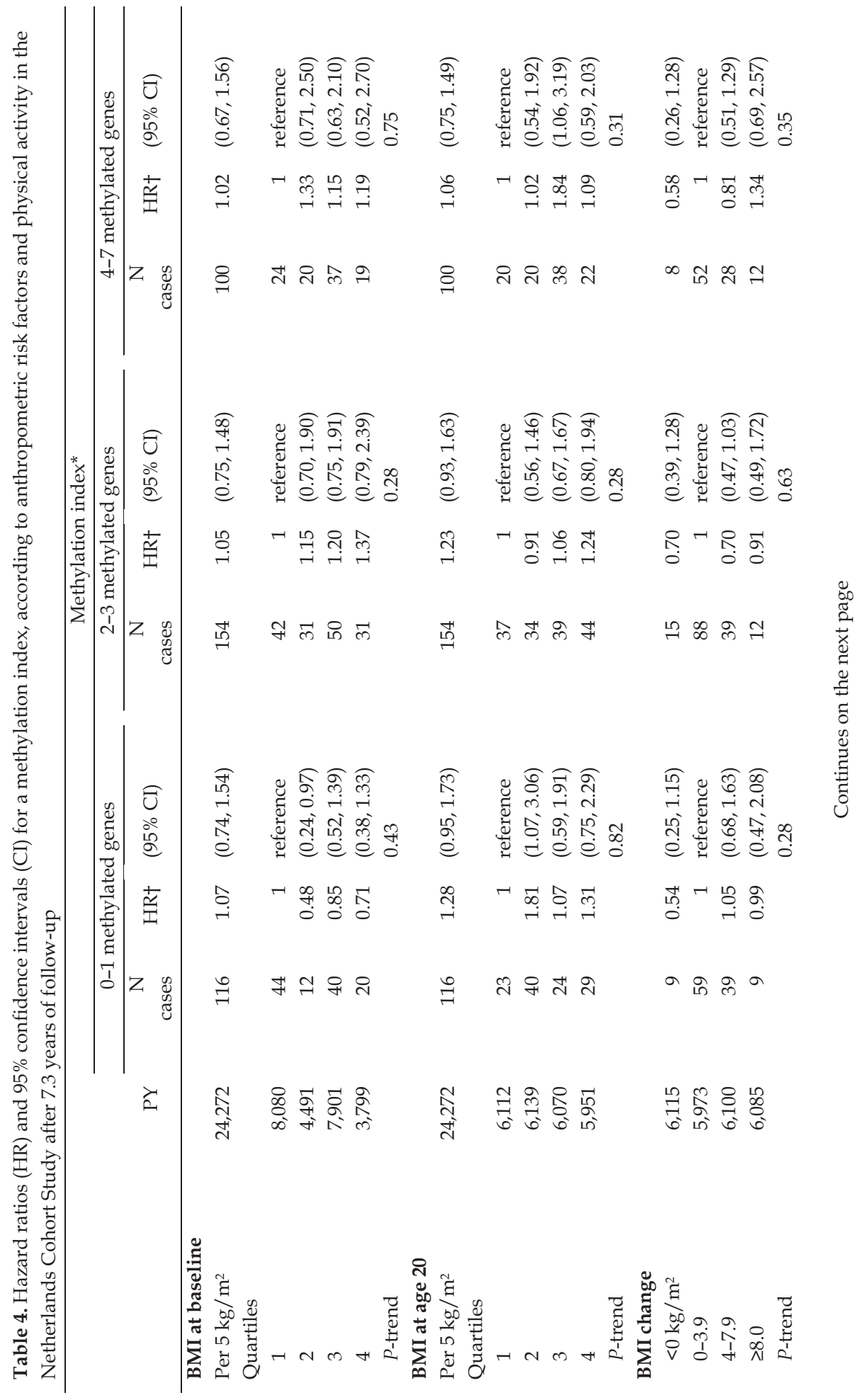




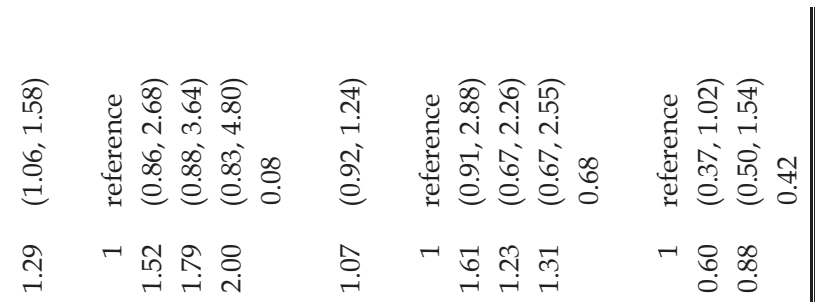

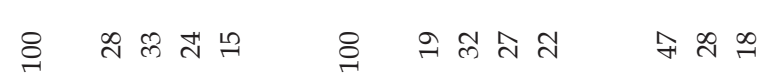

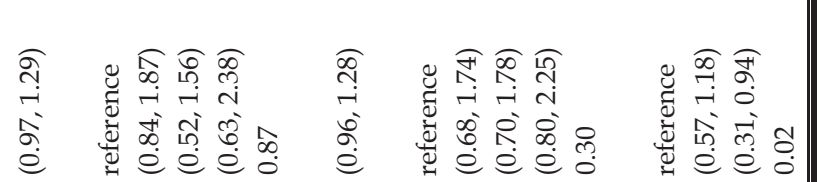

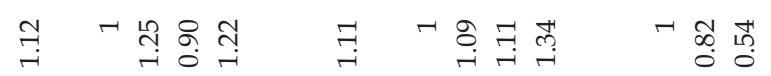

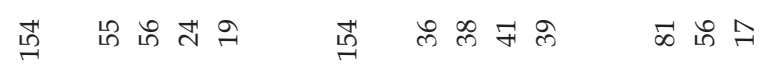

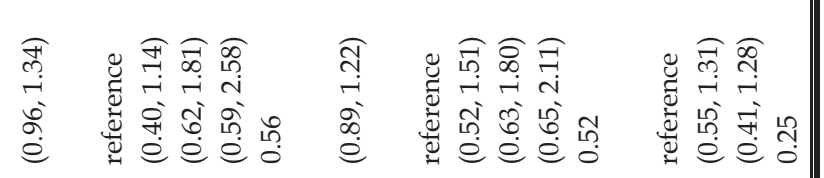

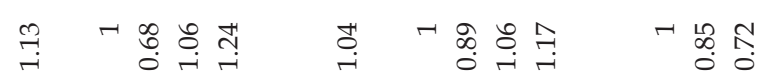

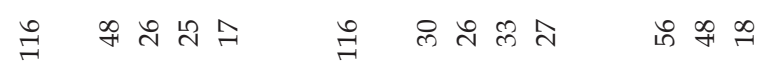

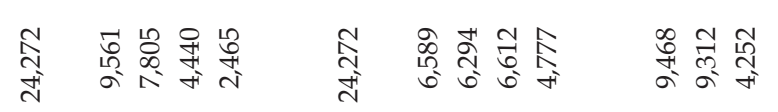

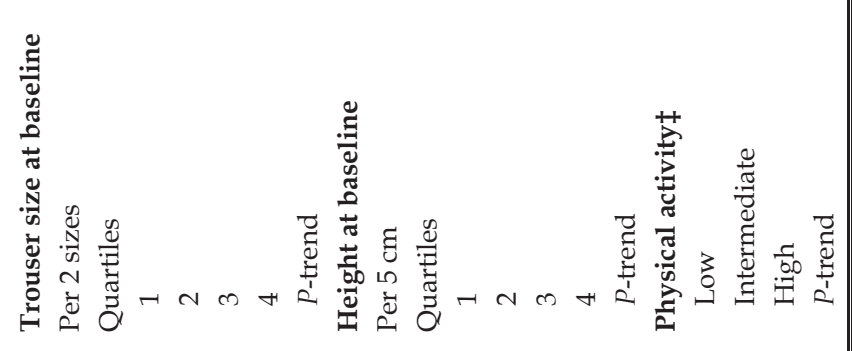

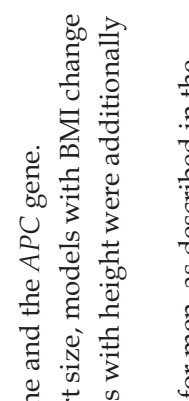

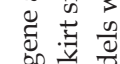

㱐部

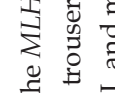

$\varsubsetneqq \pm$

矛造

की

क :

空

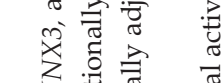

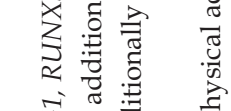

जे

0 范

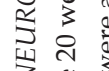

要

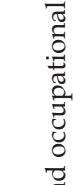

心毒泀

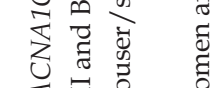

过菖

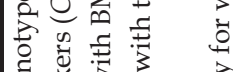

:

들

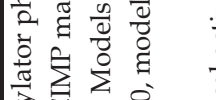

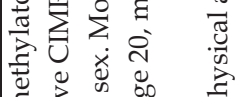

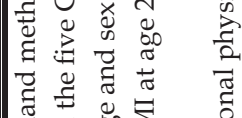

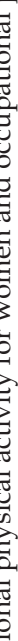
需 U. 
The NLCS utilized the Weisenberger panel of genes to define CIMP (CACNA1G, IGF2, NEUROG1, RUNX3, and SOCS1), whereas the study of Slattery et al., utilized the Classic panel (MINT1, MINT2, MINT31, p16, and $h M L H 1)$, as well as different cut-offs to define CIMP in tumors. ${ }^{36,37}$ The 'right' definition of CIMP is still a highly debated topic, as is the ideal gene panel and the appropriate method of methylation detection. ${ }^{12,38}$ While the Weisenberger panel has been validated, different markers of methylation may be more or less informative with respect to studying different exposures. The prevalence of CIMP in the NLCS population is higher than in the study by Slattery et al. (27\% vs. $11 \%) .{ }^{12}$ However, a difference in primer designs and PCR conditions may substantially change sensitivity and specificity of a particular marker for the detection of CIMP in CRC. ${ }^{39}$ Therefore, it is likely that differences in prevalence are not due to the different methods per se, but rather a difference in choice of primers. The MSP analyses that have been conducted in the NLCS have a high detection signal, and subsequently, a higher prevalence of CIMP has been observed. In the present study, we attempted to clarify our observations by constructing a methylation index with different cut-off points that included 7 genes commonly methylated in CRC.

In the present study, we considered colon and rectal tumors together to increase statistical power. A sensitivity analysis revealed that this did not bias our findings (data not shown). Furthermore, we suggest that the idea of combining sublocalizations of tumors may be acceptable when studying molecular endpoints, because this may help explain differences in etiology according to sublocalization.

We observed that BMI was associated with both CIMP tumors and non-CIMP tumors; however, after adjustment for clothing size, these associations disappeared. This is in contrast to case-cohort data suggesting that BMI is associated only with CIMP negative colon tumors and not with rectal tumors. ${ }^{11,12}$ In our study, trouser/skirt size appears to be a strong, independent predictor of both tumor subtypes, even after adjustment for BMI. This is logical, because waist circumference, an indicator of central adiposity, is a stronger predictor of CRC than BMI.1,40 When we considered associations according to a methylation index, we did not observe clear associations with respect to BMI and the degree of promoter methylation, however, we observed that trouser/skirt size was associated with the highest level of methylation. That we observed associations between trouser/skirt size and both CIMP and non-CIMP tumors suggests that central adiposity may influence $\mathrm{CRC}$ risk through both a methylation and a non-methylation pathway.

Although very few studies have considered associations between BMI and CIMP, a number have considered endpoints in the same pathway as CIMP. CRC has distinct molecular subsets, which evolve through different pathways. ${ }^{16}$ The path to a serrated adenocarcinoma appears to take one of two major routes: the traditional serrated pathway or the sessile serrated pathway. ${ }^{41}$ The sessile serrated pathway is characterized by a high degree of CIMP, BRAF V600E mutations, and ultimately develops into microsatellite instability (MSI). ${ }^{41}$ MSI may serve as a marker for CIMP or other molecular events in CRC, ${ }^{42}$ therefore, it may be informative to consider the findings of the present study in the context of that research. Two case-control studies have reported that BMI appears associated with microsatellite stable (MSS) tumors, and less with MSI tumors. ${ }^{43,44}$ Neither study reported associations according to waist circumference. In a recent pooled analysis of NLCS data and data from the Melbourne Collaborative Cohort Study, we observed similar associations, although 
the test for heterogeneity between the tumor subtypes was not statistically significant. ${ }^{45}$

There is evidence to suggest that early life exposures influence epigenetic mechanisms associated with adult disease risk. 46,47 Therefore, we also investigated associations between BMI at age 20, BMI change, height and CIMP status. Height is a marker of an aggregated fetal and childhood experience, and can be considered a proxy measure for important nutritional exposures, which affect several hormonal and metabolic axes. ${ }^{17}$ In the NLCS, we have observed that childhood and adolescent energy restriction is associated with a decreased risk of CRC later in life, ${ }^{48,49}$ which is supported by other population-based studies. ${ }^{50-52}$ We also recently reported that exposure to severe energy restriction during childhood and adolescence was associated with a decreased risk of developing a CIMP tumor. ${ }^{47}$ Furthermore, pooled data suggest that taller individuals are at greater risk of developing an MSI tumor. ${ }^{45}$ According to the present study, height is not differentially associated with the risk of CRC, however, we observed significant heterogeneity in associations with respect to BMI at age 20 and tumor subtypes. Taken together, our findings suggest that body size may differentially influence CIMP status during different periods of life, potentially affecting later CRC risk. The association between BMI at age 20 and CIMP tumors was stronger than with non-CIMP tumors, which is in line with previous findings for severe energy restriction during childhood and adolescence. Although our bootstrapping method is quite conservative, we did not observe a clear association with respect to BMI at age 20 and the methylation index and therefore we cannot rule out that the differential association with CIMP status is a chance finding. The hypothesis that timing of exposure may influence epigenetic mechanisms requires further investigation.
That we did not observe any clear associations between BMI change and risk of tumors was surprising. This may indicate that metabolic changes in fat may be more important for modulating risk over time, rather than BMI. Alternatively, considering men and women together may have attenuated these observations. Campbell et al. report that adult weight gain was associated with CRC in men, but not in women, and only with respect to individuals who gained more than $21 \mathrm{~kg}$ since age 20.43 Finally, only considering two time points may not be indicative of true BMI change.

Our findings with respect to physical activity support those of Slattery et al.,11 and suggest that high levels of daily exercise are associated with a decreased risk of both CIMP and nonCIMP tumors. Observations with respect to our methylation index suggest that physical activity may be more protective of tumors with increasing methylation, but more research is required before firm conclusions can be drawn.

Preliminary evidence suggests that molecular markers can be used to classify CRCs into distinct subtypes, which have implications for both etiology and prevention. ${ }^{42}$ Fewer tumors arise from the sessile serrated pathway compared to the traditional adenoma pathway. ${ }^{41,53}$ As overweight and obesity are such strong risk factors for $\mathrm{CRC}$, there is a possibility that these conditions may differentially influence risk through pathways and molecular mechanisms other than what we investigated here. More research is needed to clarify the association between indicators of energy balance and epigenetic mechanisms leading to CRC; preferably in a prospective cohort setting, with many cases. ${ }^{54}$ Furthermore, as the field of molecular pathological epidemiology ${ }^{55}$ continues to evolve, standardizing methods and definitions of molecular endpoints should be addressed. This will become especially critical as more opportunities for pooling data arise. 
In conclusion, our findings suggest that measures of anthropometry reflecting a large body size increase the risk of both CIMP and non-CIMP tumors, and that body fat at young age may differentially influence risk. Physical activity appears to decrease the risk of CRC regardless of these molecular subtypes. Our observations reiterate the importance of a healthy body weight with respect to general CRC prevention. 


\section{References}

1. World Cancer Research Fund / American Institute for Cancer Research. Food, Nutrition, Physical Activity, and the Prevention of Cancer: a Global Perspective. Washington DC: AICR, 2007.

2. Derks, S. et al. Integrated analysis of chromosomal, microsatellite and epigenetic instability in colorectal cancer identifies specific associations between promoter methylation of pivotal tumour suppressor and DNA repair genes and specific chromosomal alterations. Carcinogenesis 29, 434439 (2008).

3. Issa, J. P. CpG island methylator phenotype in cancer. Nat Rev Cancer 4, 988-993 (2004).

4. Sieber, O. M., Heinimann, K. \& Tomlinson, I. P. $\mathrm{M}$. Genomic instability--the engine of tumorigenesis? Nat Rev Cancer 3, 701-708 (2003).

5. Wong, J. J., Hawkins, N. J. \& Ward, R. L. Colorectal cancer: a model for epigenetic tumorigenesis. Gut 56, 140-148 (2007).

6. Samowitz, W. S. et al. Evaluation of a large, population-based sample supports a CpG island methylator phenotype in colon cancer. Gastroenterology 129, 837-845 (2005).

7. Shen, L. et al. Integrated genetic and epigenetic analysis identifies three different subclasses of colon cancer. Proc Natl Acad Sci U.S.A. 104, 1865418659 (2007).

8. Toyota, M. et al. CpG island methylator phenotype in colorectal cancer. Proc Natl Acad Sci U.S.A. 96, 8681-8686 (1999).

9. Weisenberger, D. J. et al. CpG island methylator phenotype underlies sporadic microsatellite instability and is tightly associated with BRAF mutation in colorectal cancer. Nat Genet 38, 787-793 (2006).

10. Ferguson, L. R., Karunasinghe, N. \& Philpott, M. Epigenetic events and protection from colon cancer in New Zealand. Environ Mol Mutagen 44, 3643 (2004).

11. Slattery, M. L. et al. Diet and lifestyle factor associations with CpG island methylator phenotype and BRAF mutations in colon cancer. Int J Cancer 120, 656-663 (2007).

12. Slattery, M. L. et al. Diet, physical activity, and body size associations with rectal tumor mutations and epigenetic changes. Cancer Causes Control 21, 1237-1245 (2010).

13. Hodge, D. R. et al. Interleukin 6 supports the maintenance of p53 tumor suppressor gene promoter methylation. Cancer Res 65, 4673-4682 (2005).

14. Kang, G. H. et al. Aberrant CpG island hypermethylation of chronic gastritis, in relation to ag- ing, gender, intestinal metaplasia, and chronic inflammation. Am J Pathol 163, 1551-1556 (2003).

15. Monteiro, R. \& Azevedo, I. Chronic inflammation in obesity and the metabolic syndrome. Mediators Inflamm 2010, (2010).

16. Jass, J. R. Classification of colorectal cancer based on correlation of clinical, morphological and molecular features. Histopathology 50, 113-130 (2007).

17. Okasha, M., Gunnell, D., Holly, J. \& Davey Smith, G. Childhood growth and adult cancer. Best Pract Res Clin Endocrinol Metab 16, 225-241 (2002).

18. Goldbohm, R. A. et al. Validation of a dietary questionnaire used in a large-scale prospective cohort study on diet and cancer. Eur J Clin Nutr 48, 253-65 (1994).

19. Goldbohm, R. A. et al. Reproducibility of a food frequency questionnaire and stability of dietary habits determined from five annually repeated measurements. Eur J Clin Nutr 49, 420-9 (1995).

20. Van den Brandt, P. A. et al. A large-scale prospective cohort study on diet and cancer in The Netherlands. J Clin Epidemiol 43, 285-295 (1990).

21. Van den Brandt, P. A., Schouten, L. J., Goldbohm, R. A., Dorant, E. \& Hunen, P. M. Development of a record linkage protocol for use in the Dutch Cancer Registry for Epidemiological Research. Int J Epidemiol 19, 553-558 (1990).

22. Goldbohm, R. A., van den Brandt, P. A. \& Dorant, E. Estimation of the coverage of Dutch municipalities by cancer registries and PALGA based on hospital discharge data. Tijdschr Soc Gezondheidsz 72, 80-84. (1994).

23. Brink, M. et al. K-ras oncogene mutations in sporadic colorectal cancer in The Netherlands Cohort Study. Carcinogenesis 24, 703-710 (2003).

24. Hughes, L. A., Schouten, L. J., Goldbohm, R. A., van den Brandt, P. A. \& Weijenberg, M. P. Selfreported clothing size as a proxy measure for body size. Epidemiology 20, 673-6 (2009).

25. Pylypchuk, R. D., Schouten, L. J., Goldbohm, R. A., Schouten, H. C. \& van den Brandt, P. A. Body mass index, height, and risk of lymphatic malignancies: a prospective cohort study. Am J Epidemiol 170, 297-307 (2009).

26. Simons, C. C. et al. Physical activity, occupational sitting time, and colorectal cancer risk in the Netherlands cohort study. Am J Epidemiol 177, 514-530 (2013).

27. Hettinger, T. H. , Mueller, B. H. \& Gebhard, H. Ermittlung des Arbeitsenergieumsatzes bei Dynamisch Muskulaerer Arbeit. Dortmund, Germany: Bundesarbeit fuer Arbeitsschutz, 1989. 
28. Derks, S. et al. Methylation-specific PCR unraveled. Cell Oncol 26, 291-299 (2004).

29. Herman, J. G., Graff, J. R., Myöhänen, S., Nelkin, B. D. \& Baylin, S. B. Methylation-specific PCR: a novel PCR assay for methylation status of $\mathrm{CpG}$ islands. Proc Natl Acad Sci U.S.A. 93, 9821-9826 (1996).

30. Barault, L. et al. Hypermethylator phenotype in sporadic colon cancer: study on a populationbased series of 582 cases. Cancer Res 68, 8541-8546 (2008).

31. De Vogel, S. et al. Associations of dietary methyl donor intake with MLH1 promoter hypermethylation and related molecular phenotypes in sporadic colorectal cancer. Carcinogenesis $\mathbf{2 9}$, 17651773 (2008).

32. Bolton-Smith, C., Woodward, M., Tunstall-Pedoe, H. \& Morrison, C. Accuracy of the estimated prevalence of obesity from self reported height and weight in an adult Scottish population. J Epidemiol Community Health 54, 143-148 (2000).

33. Nyholm, M. et al. The validity of obesity based on self-reported weight and height: Implications for population studies. Obes 15, 197-208 (2007).

34. Spencer, E. A., Appleby, P. N., Davey, G. K. \& Key, T. J. Validity of self-reported height and weight in 4808 EPIC-Oxford participants. Public Heal Nutr 5, 561-5 (2002).

35. Wada, K. et al. Validity of self-reported height and weight in a Japanese workplace population. Int J Obes 29, 1093-9 (2005).

36. Frazier, M. L. et al. Association of the CpG island methylator phenotype with family history of cancer in patients with colorectal cancer. Cancer Res 63, 4805-4808 (2003).

37. Park, S. J. et al. Frequent CpG island methylation in serrated adenomas of the colorectum. Am J Pathol 162, 815-822 (2003).

38. Samowitz, W. S. The CpG island methylator phenotype in colorectal cancer. J Mol Diagn 9, 281283 (2007).

39. Ogino, S. et al. Evaluation of markers for $\mathrm{CpG}$ island methylator phenotype (CIMP) in colorectal cancer by a large population-based sample. J Mol Diagn 9, 305-314 (2007).

40. Pischon, T. et al. Body size and risk of colon and rectal cancer in the European Prospective Investigation Into Cancer and Nutrition (EPIC). J Natl Cancer Inst 98, 920-931 (2006).

41. Noffsinger, A. E. Serrated polyps and colorectal cancer: new pathway to malignancy. Ann Rev Pathol 4, 343-364 (2009).

42. Ogino, S. \& Stampfer, M. Lifestyle factors and microsatellite instability in colorectal cancer: the evolving field of molecular pathological epidemiology. J Natl Cancer Inst 102, 365-7 (2010).

43. Campbell, P. T. et al. Case-control study of overweight, obesity, and colorectal cancer risk, overall and by tumor microsatellite instability status. J Natl Cancer Inst 102, 391-400 (2010).

44. Slattery, M. L. et al. Associations between cigarette smoking, lifestyle factors, and microsatellite instability in colon tumors. J Natl Cancer Inst 92, 1831-1836 (2000).

45. Hughes, L. A. et al. Body size and risk for colorectal cancers showing BRAF mutations or microsatellite instability: a pooled analysis. Int J Epidemiol 41, 1060-1072 (2012).

46. Heijmans, B. T. et al. Persistent epigenetic differences associated with prenatal exposure to famine in humans. Proc Natl Acad Sci U.S.A. 105, 17046-17049 (2008).

47. Hughes, L. A. et al. Early life exposure to famine and colorectal cancer risk: a role for epigenetic mechanisms. PloS One 4, e7951 (2009).

48. Dirx, M. J., van den Brandt, P. A., Goldbohm, R. A. \& Lumey, L. H. Energy restriction early in life and colon carcinoma risk: results of The Netherlands Cohort Study after 7.3 years of follow-up. Cancer 97, 46-55 (2003).

49. Hughes, L. A. et al. Childhood and adolescent energy restriction and subsequent colorectal cancer risk: results from the Netherlands Cohort Study. Int J Epidemiol 39, 1333-1344 (2010).

50. Frankel, S., Gunnell, D. J., Peters, T. J., Maynard, M. \& Davey Smith, G. Childhood energy intake and adult mortality from cancer: the Boyd Orr Cohort Study. BMJ 316, 499-504 (1998).

51. Svensson, E. et al. Trends in colorectal cancer incidence in Norway by gender and anatomic site: an age-period-cohort analysis. Eur J Cancer Prev 11, 489-495 (2002).

52. Svensson, E. et al. Early life events and later risk of colorectal cancer: age-period-cohort modelling in the Nordic countries and Estonia. Cancer Causes Control 16, 215-223 (2005).

53. Snover, D. C. Update on the serrated pathway to colorectal carcinoma. Hum Pathol 42, 1-10 (2011).

54. Ogino, S. et al. CpG island methylator phenotype (CIMP) of colorectal cancer is best characterised by quantitative DNA methylation analysis and prospective cohort studies. Gut 55, 1000-1006 (2006).

55. Ogino, S., Chan, A. T., Fuchs, C. S. \& Giovannucci, E. Molecular pathological epidemiology of colorectal neoplasia: an emerging transdisciplinary and interdisciplinary field. Gut 60, 397-411 (2010). 


\section{A novel classification of colorectal tumors based on microsatellite instability, the $\mathrm{CpG}$ island methylator phenotype and chromosomal instability: implications for prognosis}

Colinda CJM Simons, Laura AE Hughes, Kim M Smits, Carolina A Khalid-de Bakker, Adriaan P de Bruïne, Beatriz Carvalho, Gerrit A Meijer, Leo J Schouten, Piet A van den Brandt, Matty P Weijenberg and Manon van Engeland

Ann Oncol. 2013 Aug;24(8):2048-56. 


\section{-Abstract-}

Background: We studied the overlap between the major (epi)genomic events microsatellite instability (MSI), the CpG island methylator phenotype (CIMP), and chromosomal instability $(\mathrm{CIN})$ in colorectal cancer (CRC), and whether specific (epi)genotypes were associated with CRC-related deaths.

Methods: Molecular analyses using tumor DNA were successful in 509 CRC cases identified within the Netherlands Cohort Study in the period 1989-1993. Follow-up for the vital status until May 2005 was $100 \%$.

Results: MSI (12.6\%), CIMP-only (5.3\%), CIMP + CIN (13.4\%), CIN-only (58.2\%), and triplenegative tumors $(10.6 \%)$ differed significantly regarding tumor localization, differentiation grade, initial adjuvant therapy (AT) use, and genetic characteristics ( $P$-values $\leq 0.03)$. CIMPonly, CIMP + CIN, and triple-negative tumors, compared with CIN-only tumors, were significantly associated with a 3.67, 2.44, and 3.78-fold risk of CRC-related deaths after two-year follow-up (95\% confidence intervals (CIs): 1.70, 7.91; 1.35, 4.41; and 1.97, 7.25, respectively), but not after late follow-up. MSI tumors were borderline significantly associated with a 0.40 -fold risk of CRC-related deaths after late follow-up (95\% CI: 0.15, 1.03).

Conclusion: This is the first study to show that specific (epi)genotypes may hold a differential prognostic value that may vary over time. Although no specific treatment data were available, an explanation for the differential findings over time might be that (epi)genotypes modify therapy response. 


\subsection{Introduction}

In the pathogenesis of colorectal cancer (CRC), DNA mutations, especially due to genomic instability, chromosomal aberrations and DNA promoter hypermethylation significantly determine dysregulated gene expression contributing to tumorigenesis. Because the relative contributions of these mechanisms differ between CRCs, CRCs are classified as microsatellite unstable (MSI), CpG island methylator phenotype (CIMP), and chromosomally unstable (CIN). MSI is associated with changes in short microsatellite repeats, caused by defective mismatch repair, usually due to methylation of the MutL homolog 1 (MLH1) gene. ${ }^{1,2}$ CIMP results in transcriptional silencing of specific tumor suppressor- and DNA repair genes, including $M L H 1 .^{3-5} \mathrm{CIN}$ tumors show chromosomal gains and losses and structural rearrangements, possibly reflecting an increased mutation rate. ${ }^{6}$ The mechanisms underlying CIMP and CIN remain elusive.

MSI, CIMP, and CIN are not mutually exclusive, and although MSI and CIMP correlate well, $^{7}$ the overlap between MSI, CIMP, and $\mathrm{CIN}$ is unclear. ${ }^{8}$ Consequently, it is unknown whether specific subgroups have clinical relevance, in addition to tumor staging. ${ }^{9}$ Studies that analyzed MSI and CIMP ${ }^{10}$ or MSI and $\mathrm{CIN}^{11-16}$ have consistently shown good prognosis for MSI tumors, ${ }^{17}$ yet large CRC case series with concurrent information on MSI, CIMP, CIN, and CRC-related deaths are lacking.

Within the Netherlands Cohort Study (NLCS), we investigated the overlap between MSI, CIMP and CIN, and the association between specific (epi)genotypes and CRC-related deaths. Clinical information, P53 overexpression status, and mutation status in $A P C$, KRAS, and BRAF V600E were also available.

\subsection{Methods}

7.2.1 Population and design

CRC cases (ICD-O-1 153) were identified within the NLCS through record linkage to the population-based cancer registry and the national pathology database (PALGA). ${ }^{18,19}$ The NLCS was designed to study associations between diet and cancer, ${ }^{20}$ and was approved by the institutional review boards of the TNO Nutrition and Food Research Institute and Maastricht University. The NLCS includes 120,852 participants who completed a selfadministered questionnaire at baseline in 1986, when 55-69 years old. Participants who reported a history of cancer (excluding skin cancer) at baseline were excluded. The estimated completeness of cancer follow-up is more than $96 \% .^{21}$

Figure 1 shows the collection of tumor material from CRC cases. Sufficient DNA, isolated from formalin-fixed, paraffin-embedded (FFPE) sections after macrodissection of tumor cells, was available for 733 cases. ${ }^{22}$ Age, sex, and family history of CRC were derived from the NLCS questionnaire. Information on tumor localization, incidence date, tumor node metastasis (TNM) stage, and initial adjuvant therapy (AT) use was obtained from the cancer registry. Differentiation grade was derived from the PALGA reports. Follow-up for the vital status was carried out through linkage to the Central Bureau of Genealogy and the municipal population registries until 1 May 2005. Causes of death were retrieved from the Central Bureau for Statistics. Vital status was obtained for all cases. We excluded cases for which the cause of death was unknown $(n=4)$, cases diagnosed at autopsy (n $=6$ ), and cases who died less than 30 days after diagnosis $(\mathrm{n}=19)$. 


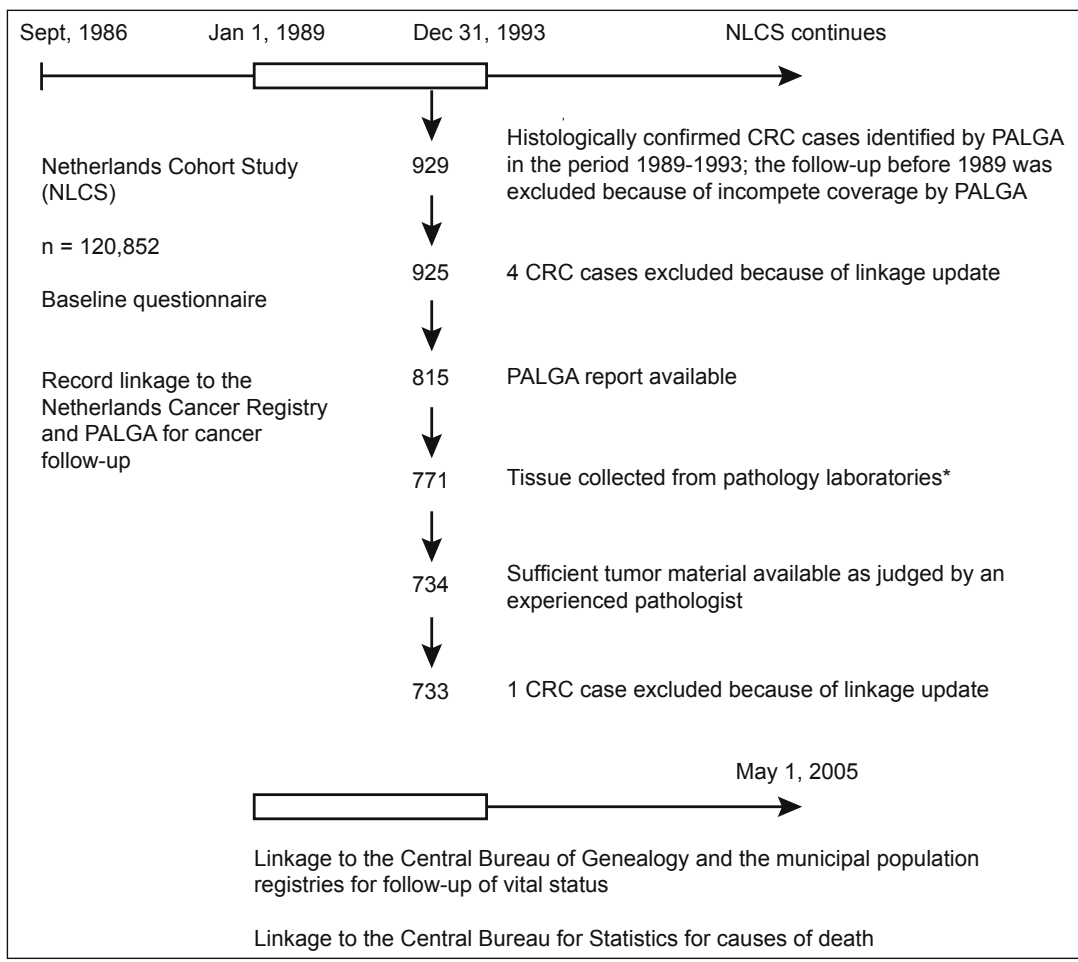

Figure 1. Flow chart of colorectal cancer cases available for analyses in the Netherlands Cohort Study.

* Tumor tissue was collected after approval by the ethical review boards of Maastricht University, the population-based cancer registry and PALGA; the pathology laboratories made available the tumor blocks between August 1999 and December 2001.

Abbreviations: CRC, colorectal cancer; PALGA, Netherlands pathology database; NLCS, Netherlands Cohort Study

\subsubsection{Epigenetic instability}

MSI. MSI was determined by a pentaplex polymerase chain reaction (PCR) using five mononucleotide repeats: BAT-26, BAT-25, NR-21, NR-22, and NR-24. Allelic size variations in three or more repeats were a marker for MSI; other tumors were classified as microsatellite stable. This method was shown to have high sensitivity and specificity. ${ }^{23}$ Analyses were successful in $90 \%$ of 733 cases.

CIMP. CIMP was defined by CpG island promoter hypermethylation of three or more out of five Weisenberger markers (CACNA1G, IGF2, NEUROG1, RUNX3, and SOCS1). ${ }^{5}$ As previously described, methylation was determined by a methylation-specific PCR (MSP) ${ }^{24,25}$ after bisulfite modification of $500 \mathrm{ng}$ DNA (Zymo Research). ${ }^{26}$ MSP is a specific, qualitative method for which the results were shown in accordance with the results of other methods. ${ }^{27}$ MSP analysis on FFPE tissue was facilitated by first amplifying the bisulfitemodified DNA (regardless of methylation status) using flanking PCR primers. All PCRs included controls for unmethylated alleles (DNA from normal lymphocytes), methylated alleles [normal lymphocyte DNA treated in vitro with SssI methyltransferase (New England Biolabs)], and a control without DNA. Analyses were successful in $81 \%, 79 \%, 79 \%$, $90 \%$, and $83 \%$ of 733 cases for CACNA1G, IGF2, NEUROG1, RUNX3, and SOCS1, respectively.

CIN. CIN was determined by multiplex ligation-dependent probe amplification (SALSA ${ }^{\circledR}$ MLPA $^{\circledR}$ reagents, MRC-Holland), ${ }^{28-30}$ after DNA purification (QIAamp ${ }^{\circledR}$ micro DNA kit, Qiagen $\mathrm{GmbH}$ ). Hundred nanograms of purified DNA were denatured at $98^{\circ} \mathrm{C}$ for $5 \mathrm{~min}$, 
after which MLPA probes were added and allowed to hybridize for $16 \mathrm{~h}$ at $60^{\circ} \mathrm{C}$ in a thermocycler. Then, $1 \mathrm{U}$ of ligase- 65 enzyme was added and ligation was allowed to proceed for $15 \mathrm{~min}$ at $54^{\circ} \mathrm{C}$. Primers, dNTPs, and Taq polymerase were added after heat inactivation of the ligase- 65 enzyme at $98^{\circ} \mathrm{C}$. PCR amplification was done for 35 cycles ( $30 \mathrm{~s}$ at $95^{\circ} \mathrm{C}, 30 \mathrm{~s}$ at $60^{\circ} \mathrm{C}$ and $1 \mathrm{~min}$ at $72^{\circ} \mathrm{C}$ ). Amplification products were quantified by capillary electrophoresis using the ABI 3730 DNA analyzer, with a LIZ-labeled internal size standard (LIZ-600, Genescan, Life Technologies Corporation, Applied Biosystems). A comparison of the sample peak pattern of case samples with that of control samples showed which sequences had copy number gains or losses.

We targeted gains in 8q23-qter, 13q14-31, and 20q13, and losses in 8p21-pter, 15q11-q21, 17p12-13, and 18q12-21 (custom-designed probe sets, MRC-Holland). Specific combinations of these abnormalities have been associated with progressed colorectal adenomas and CRC, indicating multiple CIN pathways. ${ }^{31}$ For normalization purposes, analyses included reference probes and DNA from normal FFPE colon tissue. All samples were analyzed at least in duplo. Positive controls consisted of cell lines HT29 (when targeting gains) or COLO205 (when targeting losses); negative controls consisted of cell line LS174T. Sample probe ratios were averaged across runs, and, subsequently, for probes targeting the same regions. A ratio of 1.2 or higher in $8 \mathrm{q} 23$-qter, 13q14-31, and 20q13 defined a gain; a ratio of 0.8 or lower in 8p21-pter, 15q11-q21, 17p1213 , and 18q12-21 defined a loss. CIN was defined as the presence of two or more chromosomal changes. ${ }^{31,32}$ Analyses were successful in $87 \%$ of 733 cases.
7.2.3 Gene mutation and expression analyses

APC and KRAS mutations were analyzed using a nested PCR approach, amplifying the mutation cluster region in $A P C$ and the exon 1 fragment in $K R A S$, followed by direct sequencing using the purified fragments. 22,33 Immunohistochemical staining for P53 expression was carried out according to the avidin-biotin-peroxidase complex method; positive staining of $20 \%$ or more of the tumor nuclei indicated overexpression. ${ }^{34}$ The BRAF V600E mutation was analyzed by a seminested PCR and subsequent restriction fragment length polymorphism analysis ${ }^{35} A P C$, KRAS, P53, and BRAF V600E analyses were successful in $90 \%, 100 \%, 99 \%$, and $95 \%$ of 733 cases, respectively.

\subsubsection{Classification (epi)genotypes}

MSI, CIMP, and CIN status were available for 509 CRC cases. Our classification of (epi)genotypes was hypothesis-based, firstly differentiating MSI from MSS cases, because MSI cases are universally acknowledged as a distinct subgroup concerning biology and prognosis. ${ }^{1,2,17}$ In the MSS group, we then differentiated CIMP-only, CIMP + CIN, and CIN-only tumors, because CIMP is an early event in CRC development, distinct from CIN, although overlap is possible. ${ }^{36}$ Finally, we differentiated triple-negative tumors, which have been recognized in the literature. ${ }^{8}$

7.2.5 Statistical analysis

Cause-specific survival was defined as the time from CRC diagnosis until CRC-related deaths or the end of follow-up. We estimated the influence of (epi)genotypes on causespecific survival using Kaplan-Meier curves and Wilcoxon tests. Hazard ratios and 95\% confidence intervals for CRC-related deaths were estimated using Cox regression. The proportional hazards assumption was tested 
using the scaled Schoenfeld residuals ${ }^{37}$ and by inspecting -log-log transformed survival curves. Multivariable-adjusted models included the predefined potential prognostic factors age at diagnosis, sex, tumor localization, TNM stage, differentiation grade, and initial AT use. Furthermore, analyses were stratified for TNM stage and we checked the influence of adjustment for P53 overexpression status, and mutation status in $A P C$, $K R A S$, and BRAF V600E. All analyses were conducted using Stata (Stata Corp., College Station, TX). Statistical significance was indicated by a $P$-value of $<0.05$ using two-sided tests.

\subsection{Results}

7.3.1 Prevalences (epi)genotypes

MSI, CIMP-only, CIMP + CIN, CIN-only, and triple-negative (epi)genotypes comprised $12.6 \%(n=64), 5.3 \%(n=27), 13.4 \%(n=68)$, $58.2 \%(n=296)$, and $10.6 \%(n=54)$ of cases, respectively (Figure 2). For descriptive purposes, we report that the MSI group contained 11 MSI-only, 3 MSI + CIN, 35 MSI + CIMP, and 15 triple-positive cases.

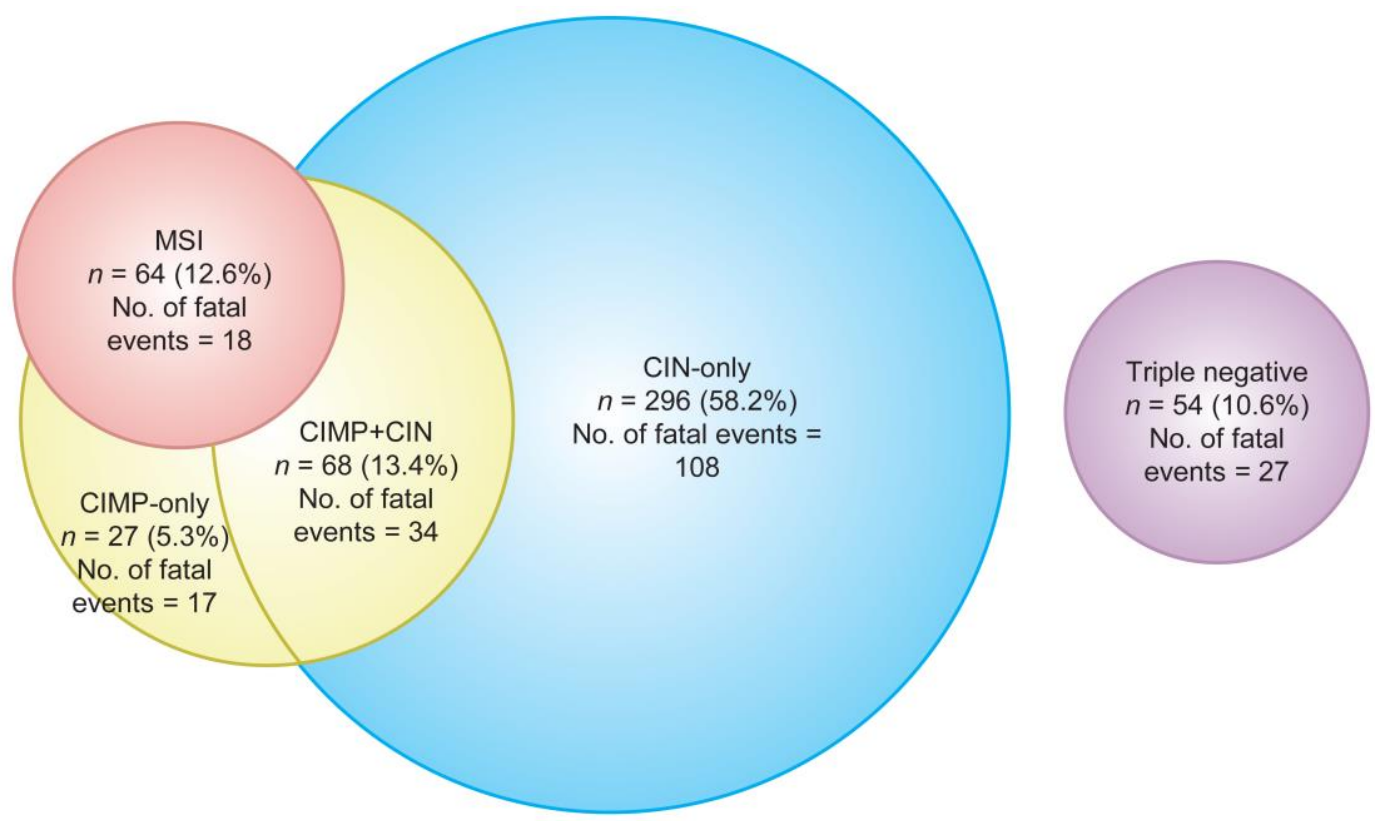

Figure 2. Venn diagram of (epi)genotypes based on MSI, CIMP, and CIN status in colorectal cancer cases from the Netherlands Cohort Study (total $\mathrm{n}=509$ ).

Abbreviations: CIMP, the CpG island methylator phenotype; CIN, chromosomal instability; MSI, microsatellite instability

\subsubsection{Clinical and genetic characteristics}

(Epi)genotypes differed significantly regarding tumor localization, differentiation grade, and initial AT use $(P$-values $\leq 0.03)$ (Table 1$)$.
MSI and CIMP-only tumors were mostly proximal colon tumors $(85.7 \%$ and $51.9 \%$, respectively), whereas CIMP + CIN and CINonly tumors were mostly distally located (distal colon to rectum: $52.3 \%$ and $82.1 \%$, respec- 


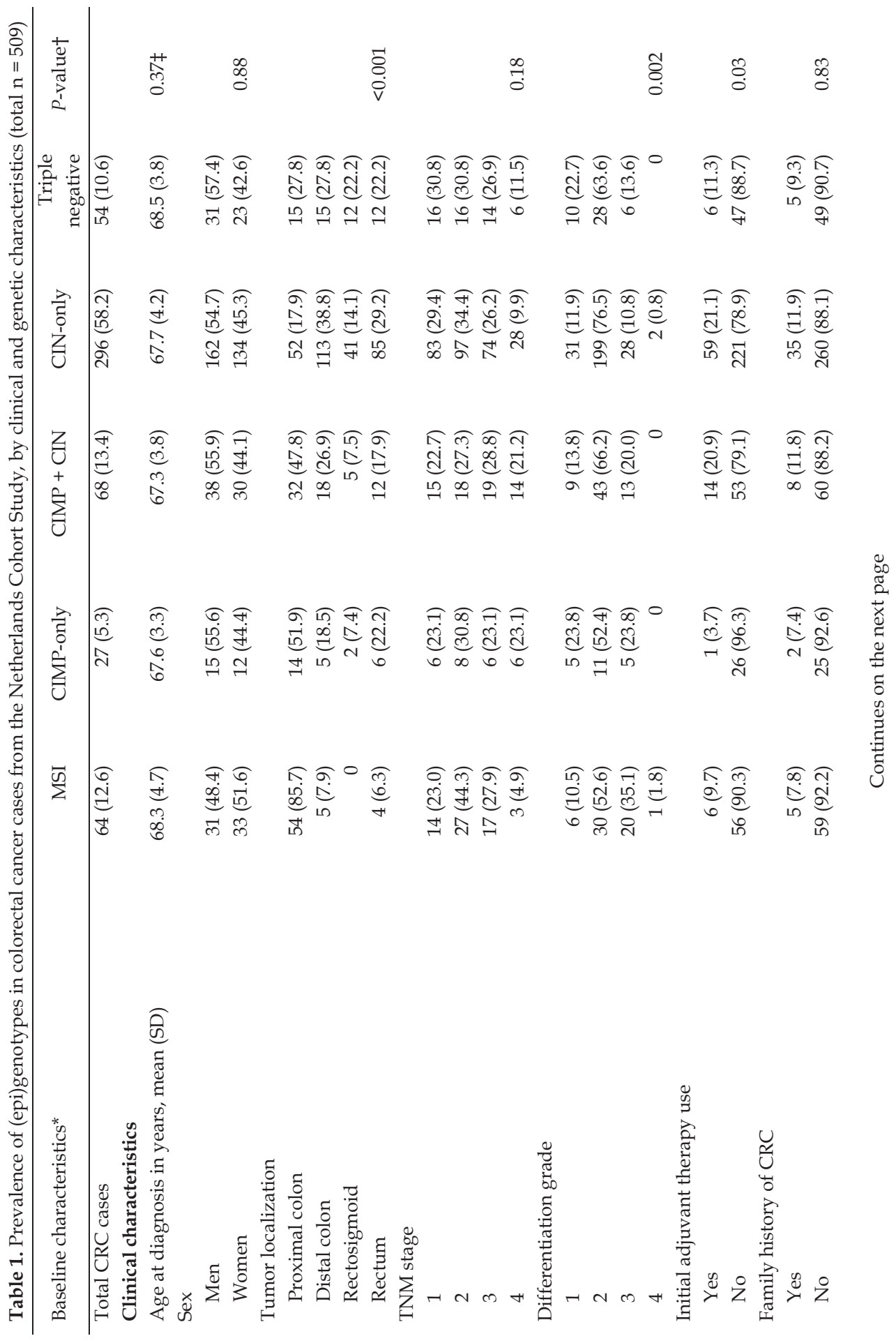




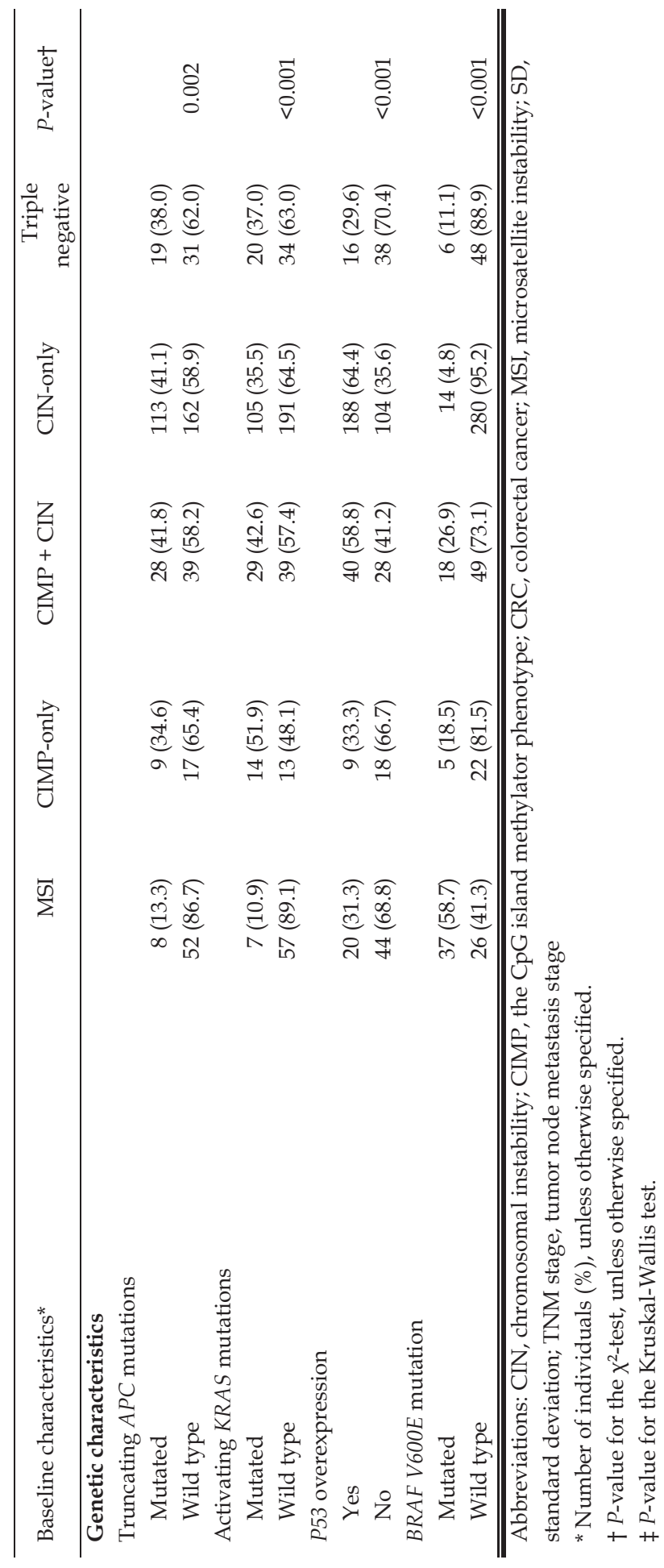


tively). Triple-negative tumors were almost equally distributed across subsites. MSI, CIMP-only, and CIMP + CIN tumors were more often poorly differentiated than other tumors $(20.0 \%-36.9 \%$ were grade 3 or 4 tumors), although the majority of tumors within all groups were classified as grade 2. CIMPonly tumors were rarely treated by initial AT (3.7\%); treatment occurred slightly more often in CIMP + CIN and CIN-only tumor groups (20.9\% and $21.1 \%$, respectively). Groups did not significantly differ regarding the age at diagnosis, sex, TNM stage, and family history of CRC.

Genetic characteristics differed significantly between (epi)genotypes ( $P$-values $\leq 0.002)$. $A P C$ and KRAS mutations occurred less often in MSI tumors $(13.3 \%$ and $10.9 \%$, respectively), but were prevalent in other groups (range: $34.6 \%-41.8 \%$ and $35.5 \%-51.9 \%$, respectively). P53 overexpression was present in all groups (range: $29.6 \%-64.4 \%$ ), but most often occurred in CIN-only tumors. The BRAF V600E mutation most often occurred in MSI tumors (58.7\%), and was particularly rare in CIN-only tumors $(4.8 \%)$.

The distributions of clinical and genetic characteristics across MSI, non-MSI, CIMP, nonCIMP, CIN, and non-CIN tumor groups are given in the Supplemental Table 1 for literature comparison.

\subsubsection{Survival}

Information on potential confounders was complete for 422 CRC cases. In this group, 169 CRC-related deaths were identified. The median follow-up was 8.4 years; the maximum follow-up was 16.3 years. Kaplan-Meier curves show that survival significantly differed between (epi)genotypes $(P<0.001)$ (Figure 3). MSI cases showed the best survival of all groups. Increasingly worse survival was observed in cases with CIN-only, CIMP + $\mathrm{CIN}$, triple-negative, and CIMP-only tumors.

In Cox models, the proportional hazards assumption was violated. As the -log-log transformed survival curves seemed to converge at two-year follow-up, we estimated hazard ratios after two-year follow-up and after late follow-up, by interacting the (epi)genotypes with the analysis time (Table 2). Unadjusted and multivariable-adjusted estimates were comparable. After early follow-up, CIMPonly, CIMP + CIN, and triple-negative cases, compared with $\mathrm{CIN}$-only cases, were at a significantly increased risk of CRC-related deaths. Multivariable-adjusted hazard ratios were 3.67 (95\% confidence intervals (CI): 1.70, 7.91), 2.44 (95\% CI: 1.35, 4.41), and 3.78 (95\% CI: 1.97, 7.25), respectively. After late followup, the hazard ratios were attenuated and no longer statistically significant. MSI cases, compared with CIN-only cases, did not differ in their risk of CRC-related deaths after early follow-up (multivariable-adjusted hazard ratio $(\mathrm{HR})=1.51,95 \% \mathrm{CI}: 0.66,3.47)$, but had a borderline significantly decreased risk after late follow-up (multivariable-adjusted $\mathrm{HR}=$ $0.40,95 \%$ CI: $0.15,1.03)$. This association became statistically significant after additional adjustment for BRAF V600E mutation status $(\mathrm{HR}=0.33,95 \% \mathrm{CI}: 0.12,0.88)$. Additional adjustment for $P 53$ overexpression status or mutation status in APC, KRAS, or BRAF V600E, showed no significant alterations (data not shown).

Kaplan-Meier curves show that survival significantly differed between (epi)genotypes within TNM stages 3 and 4 (Wilcoxon $P=$ 0.008 and $<0.001$, respectively), but not within stages 1 and 2 (Figure 4). In stratified Cox analyses, the proportional hazards assumption was violated, but numbers did not allow interacting (epi)genotypes with analysis time. Instead, we repeated the overall analyses using the stratified estimation option (Table 2). 
A

(Epi)genotypes in CRC

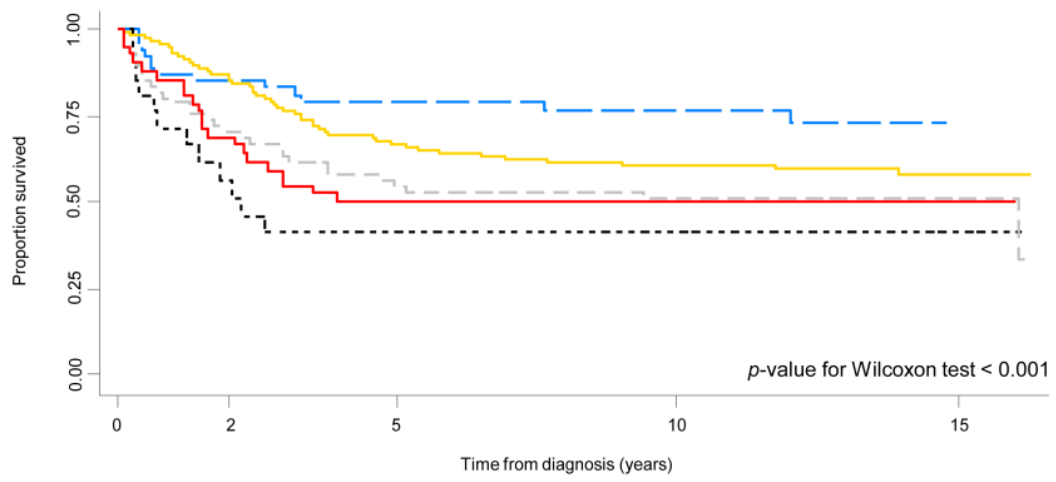

Number at risk

$\begin{array}{lccccc}\text { MSI } & 54 & 45 & 37 & 27 & 0 \\ \text { CIMP-only } & 21 & 11 & 8 & 5 & 3 \\ \text { CIMP+CIN } & 62 & 42 & 31 & 24 & 5 \\ \text { CIN-only } & 243 & 201 & 145 & 118 & 18 \\ \text { Triple negative } & 42 & 29 & 20 & 18 & 3 \\ & & & & & \end{array}$

B

(Epi)genotypes in CRC
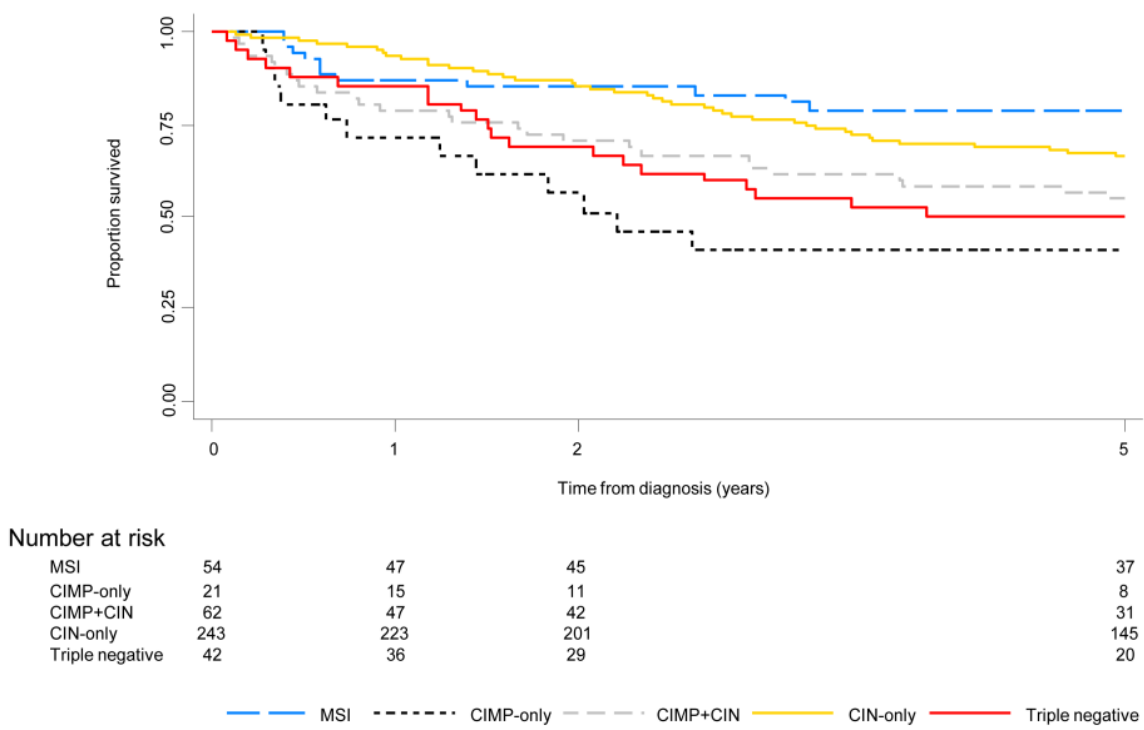

Figure 3. Cause-specific Kaplan-Meier curves according to (epi)genotypes in colorectal cancer cases from the Netherlands Cohort Study (total n = 422), showing (A) complete follow-up and (B) five-year follow-up.

Abbreviations: CRC, colorectal cancer; CIMP, the CpG island methylator phenotype; CIN, chromosomal instability; MSI, microsatellite instability 
Table 2. Hazard Ratios (HR) and 95\% confidence intervals (CI) for colorectal cancer-related deaths according to (epi)genotypes in colorectal cancer after early ( $\leq 2$ year) and late follow-up ( $>2$ years) $(n=422)$

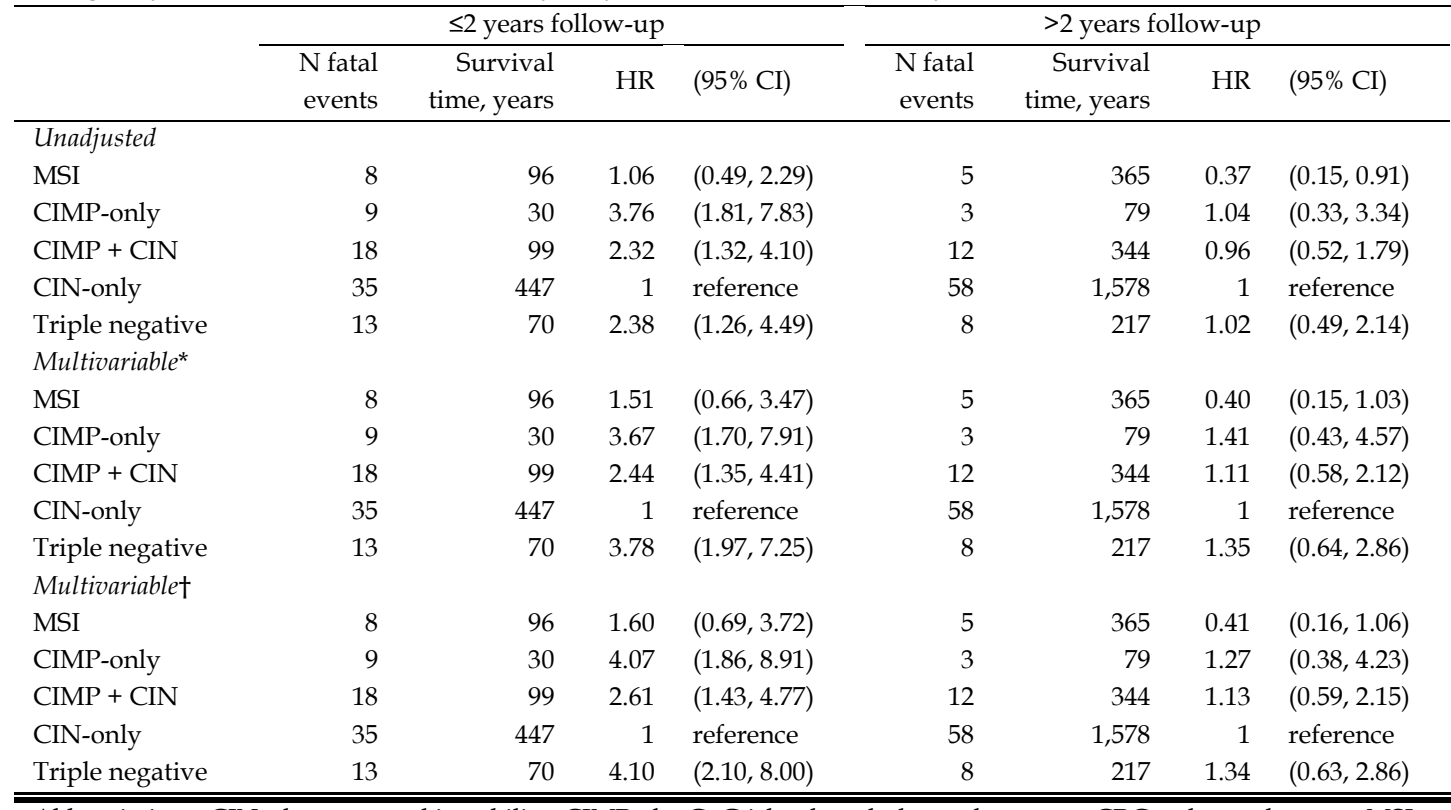

Abbreviations: CIN, chromosomal instability; CIMP, the CpG island methylator phenotype; CRC, colorectal cancer; MSI, microsatellite instability; TNM stage, tumor node metastasis stage

* The model included age at diagnosis, sex, tumor localization, TNM stage, differentiation grade, and initial adjuvant therapy use.

† The model included age at diagnosis, sex, tumor localization, differentiation grade, and initial adjuvant therapy use; stratified estimation was performed for TNM stage.

This option allowed survival curves to be disproportional between tumor stage strata, while estimating hazard ratios from a single model. ${ }^{38}$ Our results were not essentially altered.

\subsection{Discussion}

No prior studies analyzed the overlap between MSI, CIMP, and CIN, or have classified tumors accordingly. The molecular classification of tumors is complicated by different definitions in the literature. With the exception of MSI, there is no gold standard regarding gene panels, marker thresholds and techniques to define CIMP ${ }^{39}$ and CIN. We have used wellaccepted methods to define MSI, ${ }^{23}$ CIMP, 5,24,32 and $\mathrm{CIN},{ }^{28-32}$ and observed a prevalence for tumors characterized by MSI, CIMP-only, CIMP + CIN, CIN-only, and triple negative of $12.6 \%, 5.3 \%, 13.4 \%, 58.2 \%$, and $10.6 \%$, respectively. Comparably, prevalence rates of MSI and CIN in CRC were previously reported to be $\sim 15 \%$ and $\sim 65-70 \%$, respectively. ${ }^{8}$ The prevalence of CIMP ranges widely between studies $(9 \%-90 \%)$, and consensus on the definition is called for. ${ }^{39}$

Our classification, using MSI, CIMP, and CIN, was hypothesis-based. A classification by Is$\mathrm{sa}^{36}$ incorporated mutation status in APC, KRAS, P53, and BRAF V600E. We acknowledge that molecular, clinical and morphological features of CRCs displaying different instability types differ. ${ }^{40}$ However, when applying Issa's classification to our 


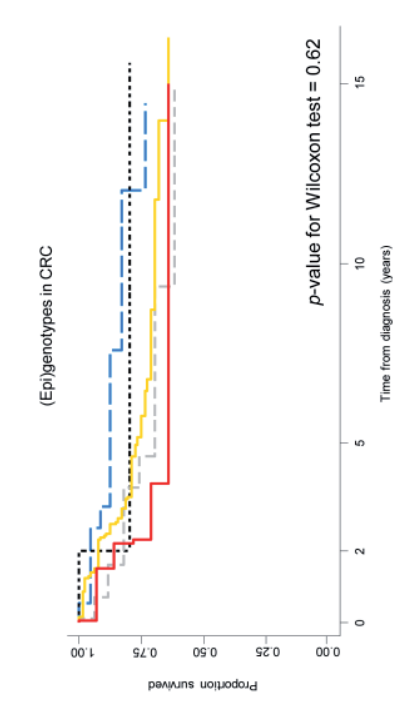

$\mathbf{m}$

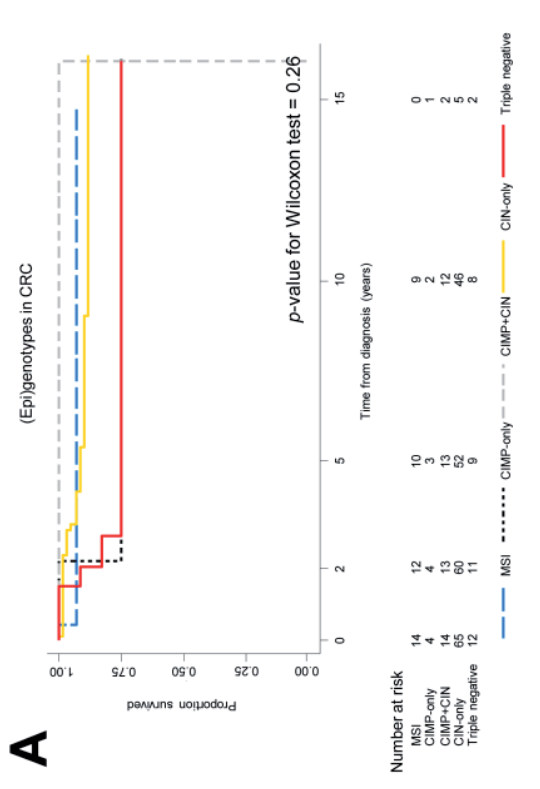

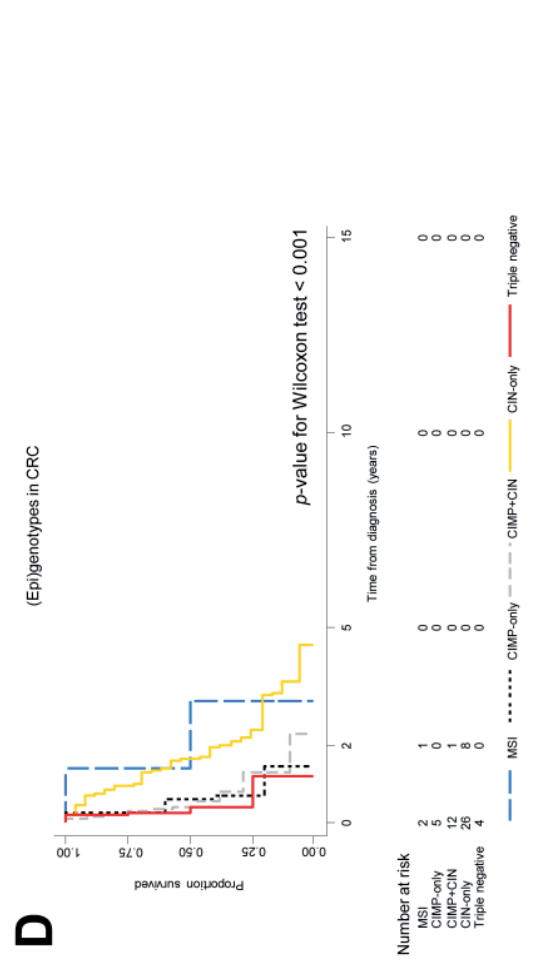

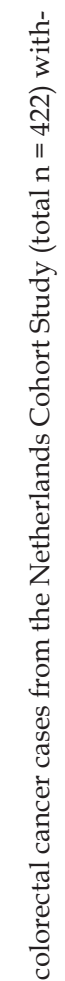

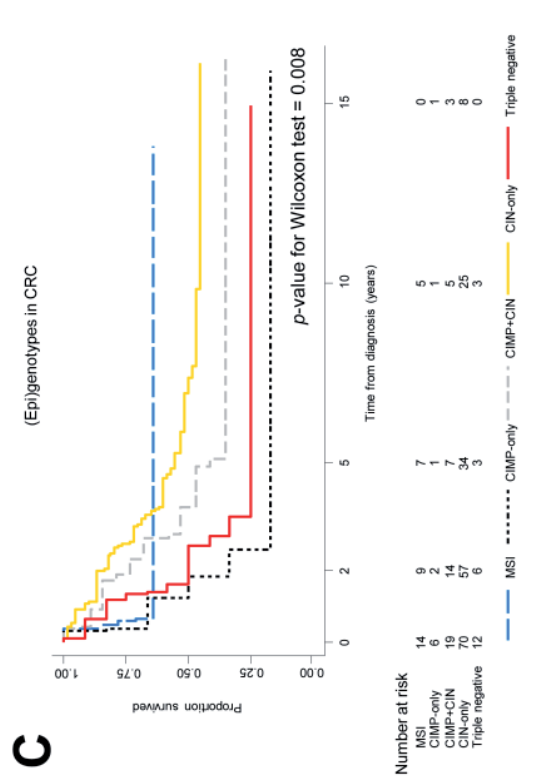

娄

品 ํํㄹㄷำ

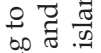

का

: 造

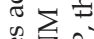

度

0

त

$\sum_{\infty}^{\infty}$

䨌

部

능응

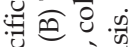

के नi讠 㐘

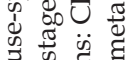

च $\sum^{\infty}$

+

总造㝴 
population, numbers in groups were low (Supplemental Figure 1), and triple-negative tumors were not distinguished. Triplenegative tumors might constitute covert CIN or CIMP tumors, ${ }^{8}$ but - unlike CIN or CIMP tumors - these did not show a predisposition for a particular subsite, nor did we observe distinct other features, e.g. a frequent family history of CRC. Therefore, the underlying biology of triple-negative tumors requires investigation. Novel genome-wide technologies appear to be promising for identifying the molecular alterations associated with this phenotype.

Next, we studied the association between (epi)genotypes and CRC-related deaths, and we observed CIMP-only, CIMP + CIN, and triple-negative cases, compared with CINonly cases, at an increased risk of CRC-related deaths after two-year follow-up, whereas MSI cases were at a decreased risk after late follow-up. Although numbers in groups were small, these results are in accordance with the literature. Generally, poor prognosis has been associated with CIN tumors ${ }^{8,41,42}$ and MSS CIMP tumors, ${ }^{10}$ whereas MSI cases show good prognosis. ${ }^{17}$ Discordant results ${ }^{43}$ may be due to heterogeneity in tumor groups, when groups are based on the overlap between only two instability types. Even when agnostically including MSI, CIMP, and CIN in a prognostic model as separate variables, the triplenegative group is present in the reference groups and will influence results. Validation of our classification and prognostic results in an independent tumor series would help corroborate the prognostic value of specific (epi)genotypes.

Most heterogeneity may be present among CIMP tumors. We characterized a considerable group by CIMP + CIN (13.4\%) and a smaller group by CIMP-only (5.3\%). These groups could reflect CIMP-low tumors, as CIMP + CIN and CIMP-only tumors were fre- quently characterized by KRAS mutations and less often by the BRAF V600E mutation, which fits with the literature on CIMP-low tumors. ${ }^{40}$ CIMP + CIN and CIMP-only cases showed poor short-term prognosis compared with CIN-only cases, whereas MSI tumors, of which the majority also had CIMP, showed good long-term prognosis. The CIMP-tumors in the MSI group could reflect CIMP-high tumors, as this group frequently exhibited the $B R A F$ V600E mutation but not KRAS mutations. ${ }^{40}$ An overruling beneficial effect of MSI on survival may be due to that MSI tumor cells are less fit to progress or metastasize, although this seems incompatible with the idea that instability drives tumor development. ${ }^{44}$ Alternatively, a survival advantage of MSI cases could be related to immune response, as MSI tumors show strong infiltration with $\mathrm{CD}^{+} \mathrm{CD} 103^{+}$lymphocytes, which have been shown less common in MSS tumors. ${ }^{44}$

Complexity is added when considering the dimension of time. We observed that the prognostic value of (epi)genotypes varied over time. Partitioning of the time axis to model the potential effects of prognostic factors in the case of non-proportional hazards is an established method..$^{45} \mathrm{~A}$ non-proportional influence of prognostic factors on hazard rates was observed in several breast cancer studies, with common patterns being that of declining predictive strength or crossover. ${ }^{46}$ Our results may have been influenced by our choice of reference group and by an influence of (epi)genotypes on therapy response. At the time of diagnosis of cases, common types of AT in the Netherlands may have been radiotherapy, especially in the case of rectal tumors, and 5-fluorouracil (5-FU) chemotherapy. We have no specific information on AT use, but as our reference group of CIN-only tumors comprised the highest proportion of rectal tumors and initial AT use, this group may have derived a survival benefit from radiotherapy treatment. If this benefit faded 
over time and was not present in other groups, this could explain the attenuation of estimates that we observed after late followup. Future studies may be encouraged to carry out subsite-specific analyses, as rectal tumors have a distinct biology; 47 however, a sensitivity analysis confined to colon cancer cases did not alter conclusions at present. Response to 5-FU was previously investigated in relation to MSI and CIMP, but results were inconsistent. ${ }^{48-50} \mathrm{CIN}$ has been proposed to confer multidrug resistance. ${ }^{51}$ Inconsistent findings may be explained by an interaction between specific (epi)genotypes and therapy type (influencing response) and the relationship between other factors and therapy response.

That adjustment for TNM stage and mutation status in key CRC genes did not essentially change the results, suggests that confounding was unlikely by these factors. As shown here and as reported previously, ${ }^{40,52}$ the $B R A F$ $V 600 E$ mutation correlates with MSI and CIMP, and mutations in KRAS, APC, and P53 correlate with CIMP and CIN. However, none of these mutations have convincing prognostic relevance, although KRAS mutations may predict poor response to treatment with epi- dermal growth factor receptor inhibitors. ${ }^{8}$ To confirm the independent prognostic effects of (epi)genotypes, future studies should stratify on the mutation status in key CRC genes and tumor stage, which would require large numbers or data pooling. Data pooling is complicated by differences in $\mathrm{CIMP}^{39}$ and CIN measurements.

Strengths of our study include the populationbased character, the nearly complete followup and the low overall prevalence of initial AT use. A limitation may be the inability to carry out stratified analyses for TNM stage because of low numbers. Still, this study is among the largest studies assessing molecular changes in CRC in relation to prognosis, and the first to shed light on the relative contributions of MSI, CIMP, and CIN to survival.

In conclusion, our data on the interplay between MSI, CIMP, and CIN showed that specific (epi)genotypes may hold differential prognostic value that may vary over time. Although no specific treatment data were available, an explanation for the differential findings over time might be that specific (epi)genotypes modify therapy response. 


\section{References}

1. Boland, C. R. \& Goel, A. Microsatellite instability in colorectal cancer. Gastroenterology 138, 20732087.e3 (2010).

2. Imai, K. \& Yamamoto, H. Carcinogenesis and microsatellite instability: the interrelationship between genetics and epigenetics. Carcinogenesis 29, 673-680 (2008).

3. Toyota, M. et al. CpG island methylator phenotype in colorectal cancer. Proc Natl Acad Sci U.S.A. 96, 8681-8686 (1999).

4. Samowitz, W. S. et al. Evaluation of a large, population-based sample supports a $\mathrm{CpG}$ island methylator phenotype in colon cancer. Gastroenterology 129, 837-845 (2005).

5. Weisenberger, D. J. et al. CpG island methylator phenotype underlies sporadic microsatellite instability and is tightly associated with BRAF mutation in colorectal cancer. Nat Genet 38, 787-793 (2006).

6. Pino, M. S. \& Chung, D. C. The chromosomal instability pathway in colon cancer. Gastroenterology 138, 2059-2072 (2010).

7. Shen, L. et al. Integrated genetic and epigenetic analysis identifies three different subclasses of colon cancer. Proc Natl Acad Sci U.S.A. 104, 1865418659 (2007).

8. Walther, A. et al. Genetic prognostic and predictive markers in colorectal cancer. Nat Rev Cancer 9, 489-99 (2009).

9. Chapuis, P. H., Chan, C. \& Dent, O. F. Clinicopathological staging of colorectal cancer: Evolution and consensus-an Australian perspective. J. Gastroenterol Hepatol 26 Suppl 1, 58-64 (2011).

10. Kang, G. H. Four molecular subtypes of colorectal cancer and their precursor lesions. Arch Pathol Lab Med 135, 698-703 (2011).

11. Georgiades, I. B., Curtis, L. J., Morris, R. M., Bird, C. C. \& Wyllie, A. H. Heterogeneity studies identify a subset of sporadic colorectal cancers without evidence for chromosomal or microsatellite instability. Oncogene 18, 7933-7940 (1999).

12. Chan, T. L. et al. Early-onset colorectal cancer with stable microsatellite DNA and near-diploid chromosomes. Oncogene 20, 4871-4876 (2001).

13. Hawkins, N. J., Tomlinson, I., Meagher, A. \& Ward, R. L. Microsatellite-stable diploid carcinoma: a biologically distinct and aggressive subset of sporadic colorectal cancer. Br J Cancer 84, 232236 (2001).

14. Jones, A. M. et al. Array-CGH analysis of microsatellite-stable, near-diploid bowel cancers and comparison with other types of colorectal carcinoma. Oncogene 24, 118-129 (2005).
15. Cai, G. et al. Clinicopathologic and molecular features of sporadic microsatellite- and chromosomal-stable colorectal cancers. Int J Colorectal Dis 23, 365-373 (2008).

16. Kets, C. M. et al. Is early-onset microsatellite and chromosomally stable colorectal cancer a hallmark of a genetic susceptibility syndrome? Int J Cancer 122, 796-801 (2008).

17. Popat, S., Hubner, R. \& Houlston, R. S. Systematic review of microsatellite instability and colorectal cancer prognosis. J Clin Oncol 23, 609-618 (2005)

18. Casparie, M. et al. Pathology databanking and biobanking in The Netherlands, a central role for PALGA, the nationwide histopathology and cytopathology data network and archive. Cell Oncol 29, 19-24 (2007).

19. Van den Brandt, P. A., Schouten, L. J., Goldbohm, R. A., Dorant, E. \& Hunen, P. M. Development of a record linkage protocol for use in the Dutch Cancer Registry for Epidemiological Research. Int J Epidemiol 19, 553-558 (1990).

20. Van den Brandt, P. A. et al. A large-scale prospective cohort study on diet and cancer in The Netherlands. J Clin Epidemiol 43, 285-95 (1990).

21. Goldbohm, R. A., van den Brandt, P. A. \& Dorant, E. Estimation of the coverage of Dutch municipalities by cancer registries and PALGA based on hospital discharge data. Tijdschr Soc Gezondheidsz 72, 80-84. (1994).

22. Brink, M. et al. K-ras oncogene mutations in sporadic colorectal cancer in The Netherlands Cohort Study. Carcinogenesis 24, 703-710 (2003).

23. Suraweera, N. et al. Evaluation of tumor microsatellite instability using five quasimonomorphic mononucleotide repeats and pentaplex PCR. Gastroenterology 123, 1804-1811 (2002).

24. Herman, J. G., Graff, J. R., Myöhänen, S., Nelkin, B. D. \& Baylin, S. B. Methylation-specific PCR: a novel PCR assay for methylation status of CpG islands. Proc Natl Acad Sci U.S.A. 93, 9821-9826 (1996).

25. Derks, S. et al. Methylation-specific PCR unraveled. Cell Oncol 26, 291-299 (2004).

26. Hughes, L. A. et al. Early life exposure to famine and colorectal cancer risk: a role for epigenetic mechanisms. PloS One 4, e7951 (2009).

27. Barault, L. et al. Hypermethylator phenotype in sporadic colon cancer: study on a populationbased series of 582 cases. Cancer Res 68, 8541-8546 (2008).

28. Schouten, J. P. et al. Relative quantification of 40 nucleic acid sequences by multiplex ligation- 
dependent probe amplification. Nucleic Acids Res 30, e57 (2002).

29. Postma, C. et al. Chromosomal instability in flat adenomas and carcinomas of the colon. J Pathol 205, 514-521 (2005).

30. Jankowski, S., Currie-Fraser, E., Xu, L. \& Coffa, J. Multiplex ligation-dependent probe amplification analysis on capillary electrophoresis instruments for a rapid gene copy number study. J Biomol Tech 19, 238-243 (2008).

31. Hermsen, M. et al. Colorectal adenoma to carcinoma progression follows multiple pathways of chromosomal instability. Gastroenterology $\mathbf{1 2 3}$, 1109-1119 (2002).

32. Derks, S. et al. Integrated analysis of chromosomal, microsatellite and epigenetic instability in colorectal cancer identifies specific associations between promoter methylation of pivotal tumour suppressor and DNA repair genes and specific chromosomal alterations. Carcinogenesis 29, 434439 (2008).

33. Lüchtenborg, M. et al. APC mutations in sporadic colorectal carcinomas from The Netherlands Cohort Study. Carcinogenesis 25, 1219-1226 (2004).

34. Bongaerts, B. W. C. et al. Alcohol consumption and distinct molecular pathways to colorectal cancer. Br J Nutr 97, 430-434 (2007).

35. Sieben, N. L. et al. Clonal analysis favors a monoclonal origin for serous borderline tumours with peritoneal implants. J Pathol 210, 405-411 (2006).

36. Issa, J. P. Colon cancer: it's CIN or CIMP. Clin Cancer Res 14, 5939-40 (2008).

37. Schoenfeld, D. Partial residuals for the proportional hazards regression model. Biometrika 69, 239-241 (1982).

38. Cleves, M., Gould, W., Gutierrez, R. \& Marchenko, Y. Introduction to Survival Analysis Using Stata. College Station, Texas: Stata Press, 2008.

39. Hughes, L. A. et al. The CpG island methylator phenotype in colorectal cancer: progress and problems. Biochim Biophys Acta 1825, 77-85 (2012).

40. Jass, J. R. Classification of colorectal cancer based on correlation of clinical, morphological and molecular features. Histopathology 50, 113-30 (2007).
41. Sheffer, M. et al. Association of survival and disease progression with chromosomal instability: a genomic exploration of colorectal cancer. Proc Natl Acad Sci U.S.A. 106, 7131-7136 (2009).

42. Furlan, D. et al. Hierarchical clustering analysis of pathologic and molecular data identifies prognostically and biologically distinct groups of colorectal carcinomas. Mod Pathol 24, 126-137 (2011).

43. Ogino, S. et al. CpG island methylator phenotype, microsatellite instability, BRAF mutation and clinical outcome in colon cancer. Gut 58, 90-96 (2009).

44. Ward, R. L. et al. Adverse prognostic effect of methylation in colorectal cancer is reversed by microsatellite instability. J Clin Oncol 21, 37293736 (2003).

45. Ahmed, F. E., Vos, P. W. \& Holbert, D. Modeling survival in colon cancer: a methodological review. Mol Cancer 6, 15 (2007).

46. Hilsenbeck, S. G. et al. Time-dependence of hazard ratios for prognostic factors in primary breast cancer. Breast Cancer Res Treat 52, 227-237 (1998).

47. Li, F. Y. \& Lai, M. D. Colorectal cancer, one entity or three. J Zhejiang Univ Sci B 10, 219-29 (2009).

48. Iacopetta, B., Kawakami, K. \& Watanabe, T. Predicting clinical outcome of 5-fluorouracil-based chemotherapy for colon cancer patients: is the CpG island methylator phenotype the 5fluorouracil-responsive subgroup? Int $\mathrm{J} \mathrm{Clin} \mathrm{On}$ col 13, 498-503 (2008).

49. Des Guetz, G. et al. Does microsatellite instability predict the efficacy of adjuvant chemotherapy in colorectal cancer? A systematic review with meta-analysis. Eur J Cancer 45, 1890-1896 (2009).

50. Guastadisegni, C., Colafranceschi, M., Ottini, L. \& Dogliotti, E. Microsatellite instability as a marker of prognosis and response to therapy: a metaanalysis of colorectal cancer survival data. Eur J Cancer 46, 2788-2798 (2010).

51. Lee, A. J. et al. Chromosomal instability confers intrinsic multidrug resistance. Cancer Res 71, 1858-1870 (2011).

52. Issa, J. P. The epigenetics of colorectal cancer. Ann NY Acad Sci 910, 140-153; discussion 153-155 (2000). 


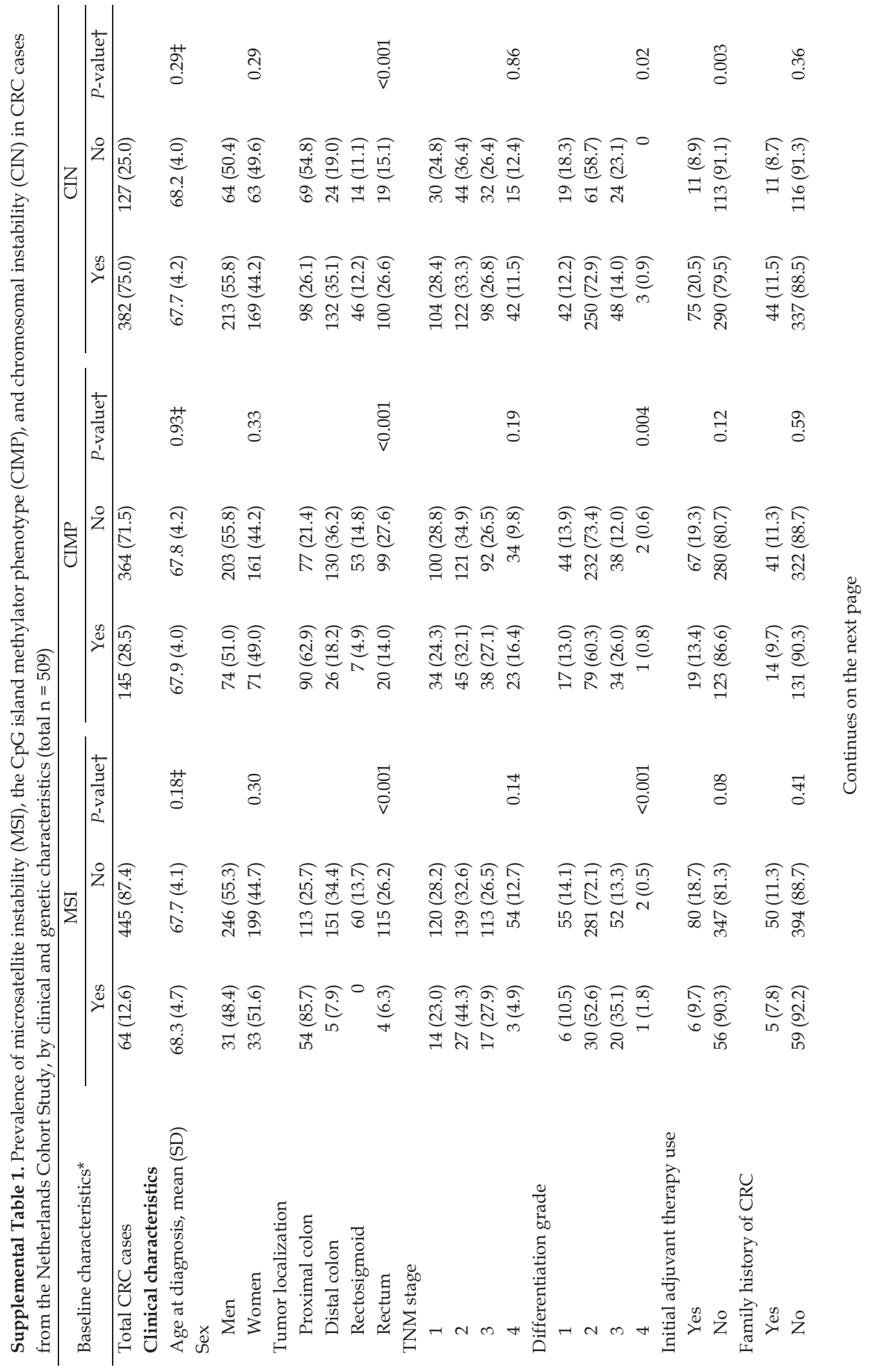




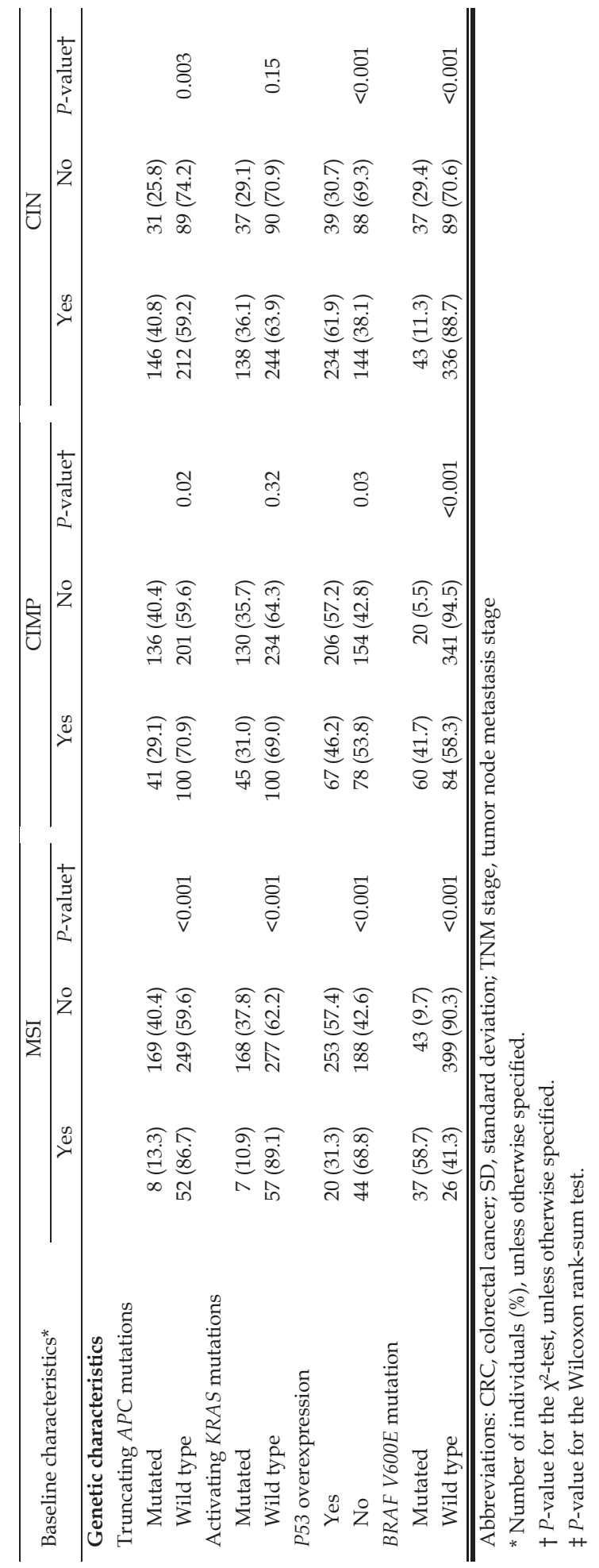




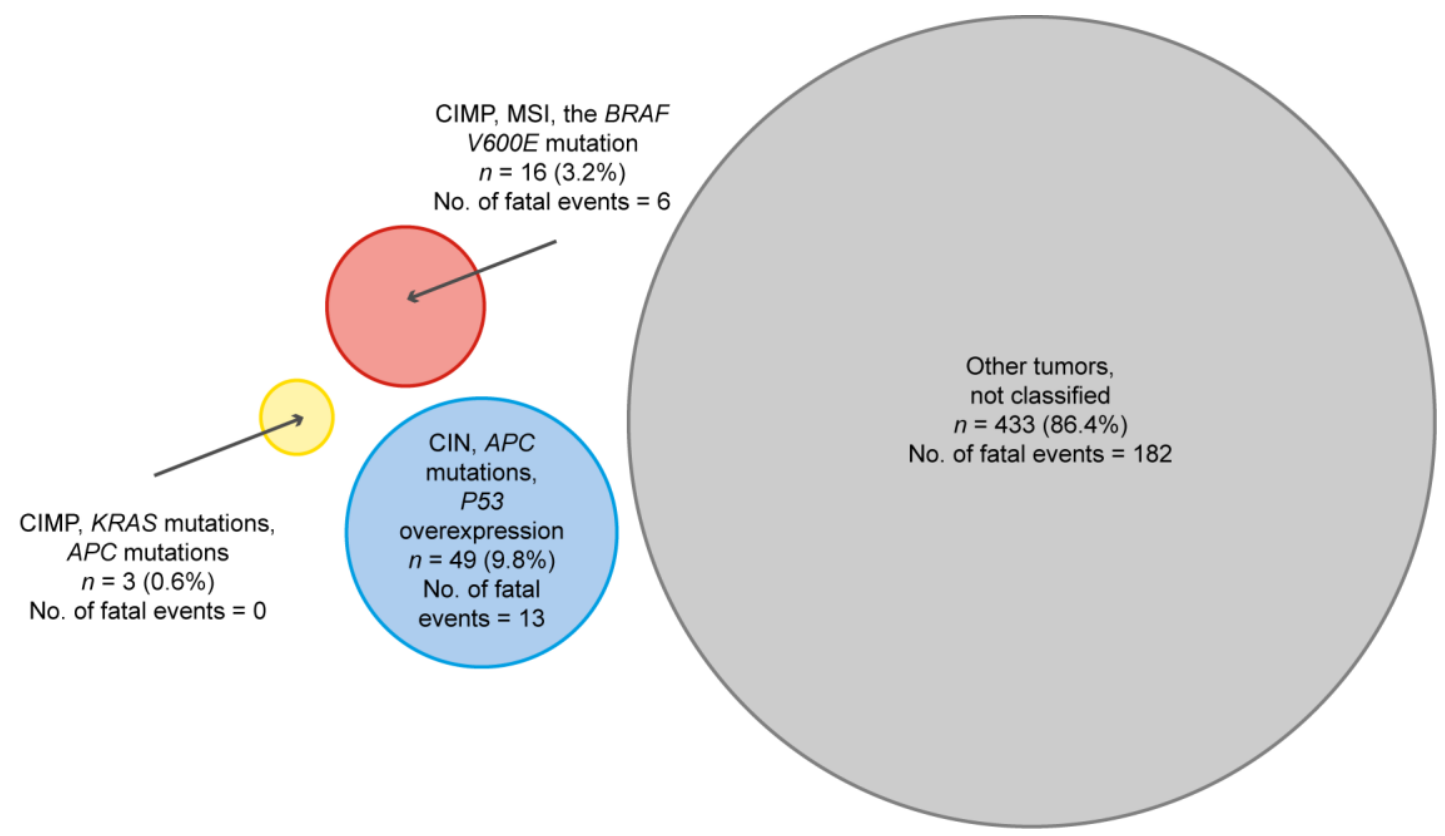

Supplemental Figure 1. Venn diagram of (epi)genotypes in colorectal cancer cases from the Netherlands Cohort Study, as based on the hypothesized pathways to colorectal cancer by Issa ${ }^{10}$ (total $n=501^{*}$ ).

* The total number of cases differs from that in Figure 2 because of missing data on P53 overexpression status and mutation status in APC, KRAS, and BRAF V600E.

Abbreviations: CIMP, the CpG island methylator phenotype; CIN, chromosomal instability; MSI, microsatellite instability 
8

\title{
General discussion
}

\author{
Colinda CJM Simons
}


The general discussion of this thesis is divided into two parts: A and B. Since chapter 4 is a central chapter in this thesis, part A concerns a perspective on gene-environment interaction studies in colorectal cancer. Part B concerns a brief discussion relating to the main findings described in this thesis. 


\section{Gene-environment interaction studies in colorectal cancer}

In this perspective, it is illustrated that myriad approaches have been used to study geneenvironment interactions in colorectal cancer when considering aspects such as the study design, the gene and SNP selection strategy, and the mode of analysis (e.g. single SNPs, haplotypes or by cumulative number of risk variants). We furthermore explore a novel SNP selection strategy based on genome-wide association studies and discuss statistical techniques to study higher-order interactions.

\section{A.1 Introduction}

The study of gene-environment (GxE) interactions in colorectal cancer (CRC) is promising because it may shed light on the pathways linking dietary and lifestyle factors to CRC. ${ }^{1}$ In the field of GXE interaction studies, a distinction can be made between hypothesisbased studies and gene-environment-wide interaction (GEWI) studies. While the first are apt for corroborating or refuting existing hypotheses, GEWI studies may identify novel hypotheses and genetic risk factors. Although there is appeal to the possibility of new, unforeseen discoveries, GEWI studies are associated with specific challenges. These challenges relate to adjusting for potential confounders, the impact of population structures, modelling changes in exposures over time, and power and sample size. ${ }^{2}$ With respect to the latter, it has been described that at least a four-fold larger sample size is needed for the detection of an interaction effect as compared to a marginal effect of similar magnitude. ${ }^{1,3}$ In the presence of imperfect exposure data (which is inevitable at some level) and small interaction effects, the required sample sizes can thus become very large. Pooling of several large case-control or cohort studies enables the conduct of GEWI studies, but may pose problems if dietary and lifestyle exposures were measured through different methods. In addition, a layer of detail may be lost if not all studies have available information on specific aspects of exposure - e.g. waist circumference as a measure of abdominal fatness in addition to weight and height - or timing of exposures.

Here, we propose that a hypothesis-based approach for GxE interaction studies in CRC is worthwhile if using background knowledge on biological mechanisms to disease. Helpful tools to pinpoint the genes of interest in hypothesis-based studies are pathway databases, such as the KEGG database. ${ }^{4}$ However, proper SNP selection remains a challenge. In this paper, we review several GxE interaction studies in CRC for the SNP selection strategy and statistical methods used. We furthermore explore a novel approach for SNP selection, and discuss possible methods for analyzing higherorder interactions between genetic variants and dietary and lifestyle factors. The analysis of higher-order interactions may be more attainable within hypothesis-based studies as opposed to GEWI studies due to being computationally intensive.

\section{A.2 GxE interaction studies in CRC}

A Pubmed search using the search terms "gene-environment interaction" and "colorectal cancer" yielded 73 articles published up until May 2013. Eighteen were original, English-language articles describing GxE interactions in relation to CRC risk (Table 1). Although the search was incomplete, it emerges from Table 1 that myriad methods have been applied when considering the agnostic or hypothesis-based character of the study, the design, including possible two-step approaches, the gene and SNP selection method, the analysis mode, the types of interaction tested (multiplicative, additive or both), and the 


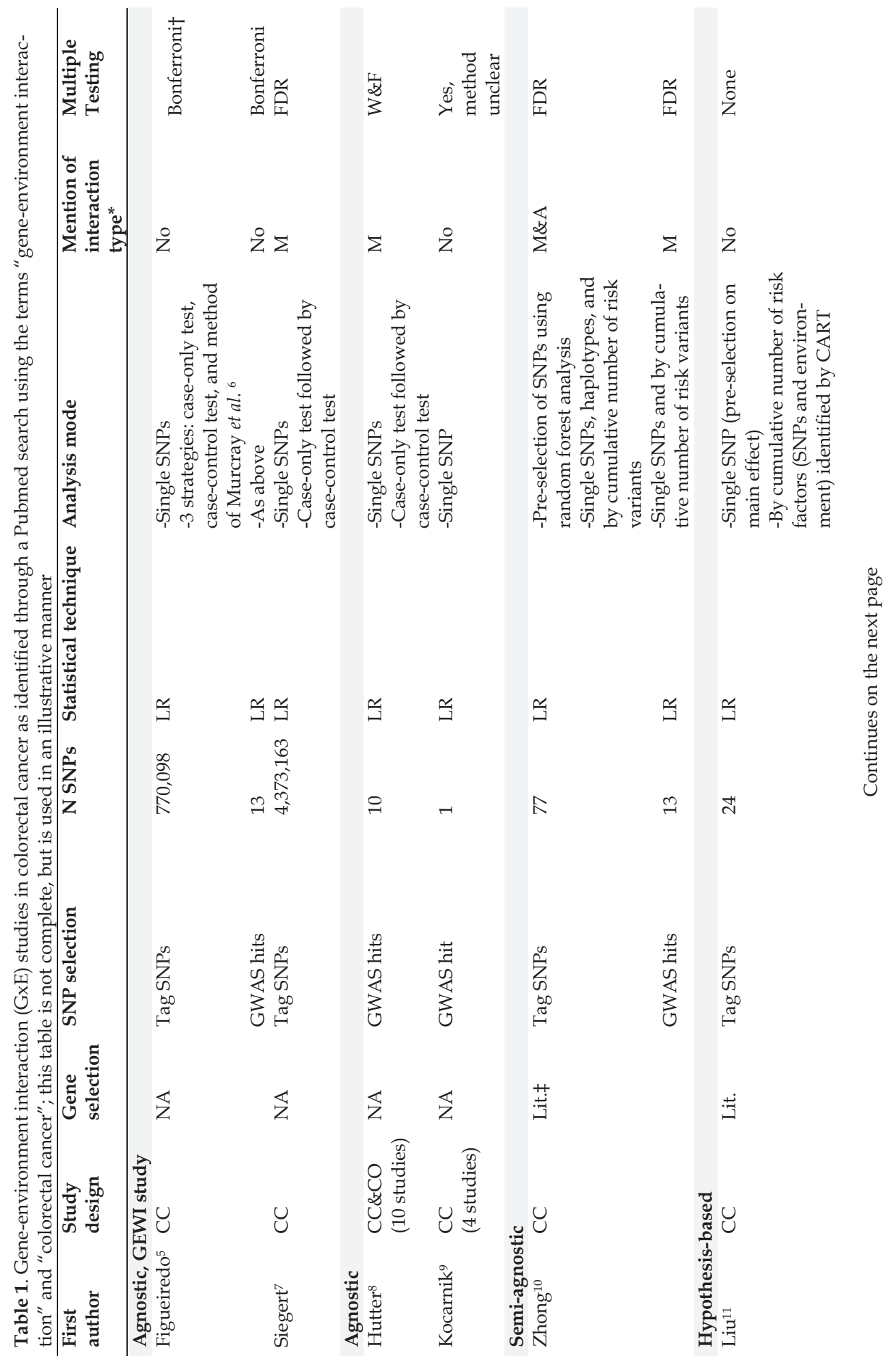




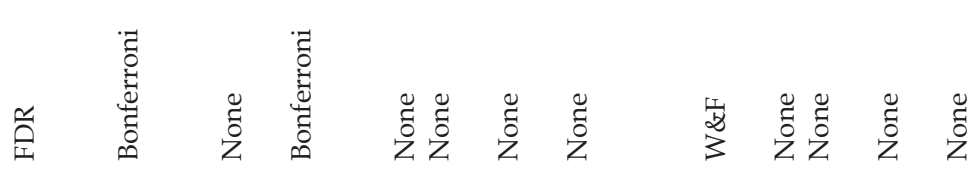

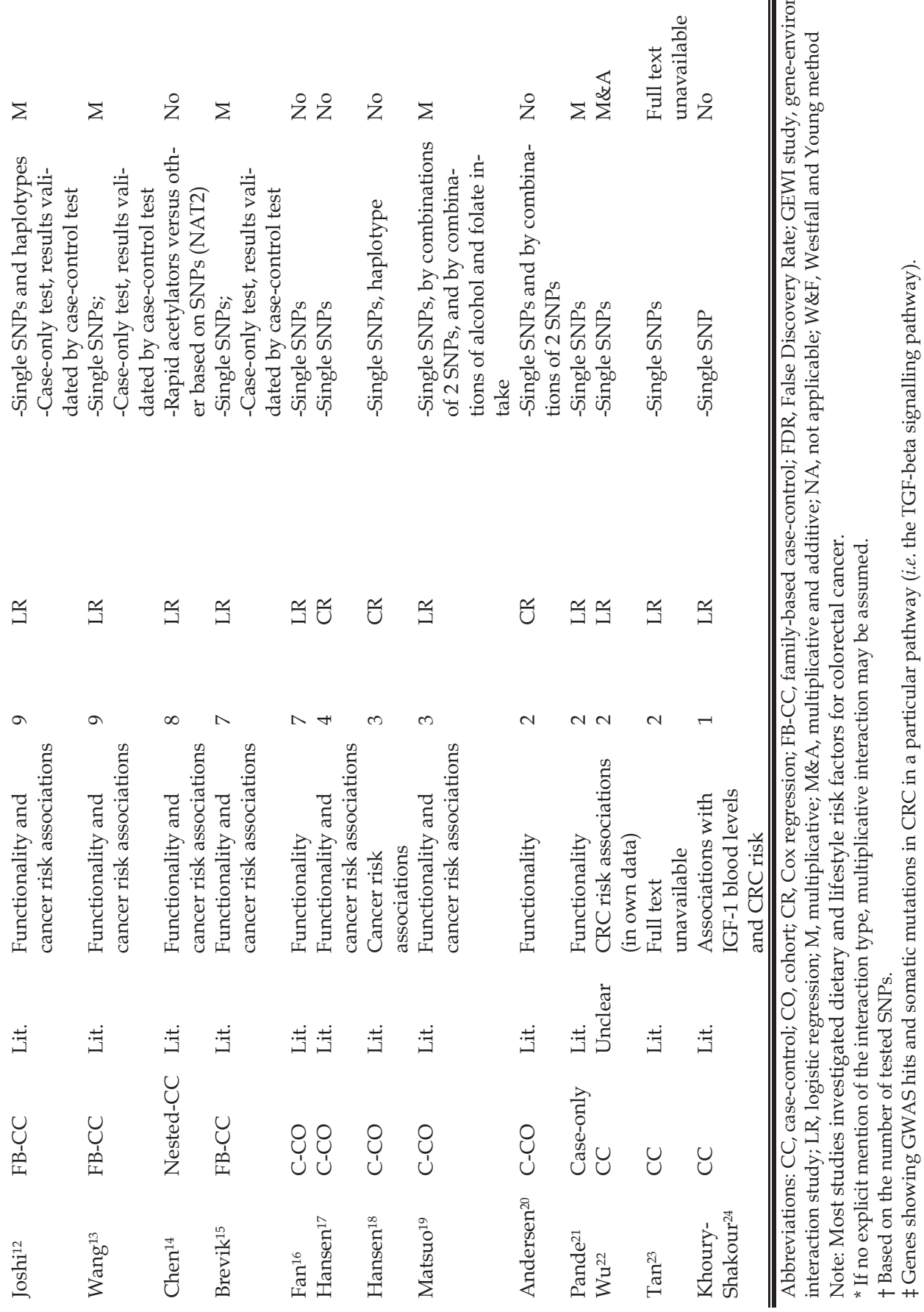


method used to correct for multiple testing. However, despite this heterogeneity, it also emerges from Table 1 that most studies were hypothesis-based, selecting genes from literature and, in these genes, selecting tagging SNPs or SNPs that had shown functional significance or cancer risk associations. One study by Siegert et al. ${ }^{7}$ qualified as a GEWI study. A second GEWI study, the only other in CRC, was cited by Siegert et al., but did not turn up in our search because it concerned cases with microsatellite stable and microsatellite instable-low CRC tumors. ${ }^{5}$ This study by Figueiredo et al. has been added to Table 1 . Both GEWI studies also investigated GxE interactions for previously reported GWAS hits; the same was done by three other studies. ${ }^{8-10}$

With respect to the design, there are advantages and disadvantages to each represented in Table 1. The case-only design has been proposed as an efficient alternative to the case-control design if the aim is to assess interaction effects. Case-only odds ratios can be interpreted as the multiplicative interaction between the gene and the environment under the assumption that these are independent of each other in the population. Trade-offs include that one cannot assess any other than a departure from multiplicativity, nor can one assess independent effects of the gene and the environment. ${ }^{25}$ Nested case-control or casecohort studies are efficient alternatives to a full cohort study. While case-control studies may be equally efficient and suited to study rare diseases, selection and information bias are less likely to occur in nested case-control and case-cohort studies because dietary and lifestyle exposures were measured in individuals still at risk of the disease. There is, however, the possibility of confounding by population stratification. Population stratification refers to differences in allele frequencies between cases and controls due to a systematic difference in ancestry, which may lead to false-positive SNP-disease associations. ${ }^{26}$ Bias due to population stratification is thought to be least likely in family-based designs, but the ascertainment of relatives as compared to non-relatives is generally logistically more difficult to accomplish.

With respect to the SNP selection strategies used, two-stage approaches were common when larger SNP numbers were selected, usually tagging SNPs (Table 1). It is beyond the scope of this paper and beyond our specific expertise to discuss all two-step approaches and GxE interaction tests that have been proposed in the literature [excellent discussions are available in e.g. ${ }^{27}$ and ${ }^{2}$. However, the essence of these approaches is to screen for SNPs that most likely interact with dietary and lifestyle factors, and then test GxE interactions for these SNPs. For example, the studies in Table 1 used as screening tools G-E association tests in cases $7,12,13,15$ or in cases and controls (Murcray's two-step procedure $\left.{ }^{6}\right),{ }^{5}$ marginal association tests, ${ }^{11}$ and tree-based techniques. ${ }^{10,11}$ Such two-step procedures increase power by reducing the multiple testing burden and may suppress genotyping expenses. Inspired by these approaches, it is our view that, with the vast number of hypothesisbased studies and GWAS already published, it may be possible to screen for SNPs that most likely interact with the environment by employing intelligent SNP selection strategies. We will discuss the possibilities next.

\section{A.3 SNP selection in hypothesis-based studies}

En route of a hypothesis-based GxE interaction study, having identified the pathway(s) and genes of interest, there is a crossroads at which one can decide to select tagging SNPs, in order to cover the genetic variation in genes, or select SNPs based on previously reported associations with relevant endpoints in the literature. When selecting tagging SNPs, all SNPs are considered equal in relation to 
the disease, but it is likely that not all SNPs are equal, and so often screening or filtering steps are included in the analysis phase, as discussed above. Selecting SNPs from literature allows direct prioritization of SNPs according to the level of evidence, but a problem may be false-positive results, ${ }^{28}$ which is why there is a need for replication and metaanalyses in epidemiological practice. A side effect of this may be that we find ourselves more often re-investigating SNPs showing significant associations as opposed to null associations, which may result in publication bias. Other (relevant) SNPs may never be studied at all. Still, welcome initiatives in terms of summarizing evidence are databases such as the CRCgene database. This database includes meta-analyses for genetic variants investigated in relation to CRC in four or more independent case-control samples, and presents results according to biological pathways. ${ }^{29}$ In order to select SNPs most likely interacting with dietary or lifestyle factors, evidence regarding CRC could be complemented with evidence from other lines of research relating to the dietary or lifestyle exposure of interest, or relating to diseases that share the dietary or lifestyle exposure as a risk factor. However, such a strategy depends on the availability of literature, and will not always be feasible.

\section{Making the most of GWAS}

With respect to SNP-disease and SNPphenotype associations, overly complete repositories already exist. These GWAS repositories may be an under-utilized legacy in terms of SNP selection, potentially harboring many false-negatives just beneath the genome-wide significance level, which is customarily set at $5^{\star} 10^{-8}$. SNPs that surpass the stringent genome-wide significance level in GWAS are thought to be likely true-positives, pointing towards biologically important genes or gene regulatory mechanisms with respect to disease. ${ }^{30,31}$ For an overview of GWAS hits in CRC, see Supplemental Table 1. However, it remains to be seen whether these SNPs are the SNPs interacting with dietary and lifestyle factors per se. For example, Figueiredo et al. ${ }^{5}$ and Kocarnik et al. ${ }^{9}$ found no significant GxE interactions with GWAS hits, and Siegert et al. ${ }^{7}$ and Hutter et al. ${ }^{8}$ found only one interaction between SNP rs1944511 and overweight and between SNP rs16892766 and vegetable consumption, respectively (Table 1). These observations prompt the idea that SNPs just below the genome-wide significance level could be interesting for the study of GxE interactions, because SNP-disease associations may be attenuated for SNPs interacting with the environment, when investigating marginal associations rather than joint effects of genes and the environment. To screen for SNPs likely involved in GxE interactions in CRC through existing GWAS, we propose the following novel strategy for consideration (see also Figure 1):

1. Select pathway(s) and genes of interest using prior knowledge.

2. Retrieve SNPs in and near (e.g. $10 \mathrm{~kb}$ upand downstream of) the selected genes from the 'top region' of a CRC GWAS.

3. Overlay the results from step 2 with results from a GWAS for a relevant trait, e.g. overweight, or a disease with shared risk factors: the choice should be relevant with respect to the dietary and lifestyle exposures of interest. Then, retain SNPs from step 2 which are also in the top region of this second GWAS.

\section{Prioritize SNPs using prior knowledge.}

In steps 2 and 3, GWAS may be used that were conducted in populations of similar ancestry as the new study population, in order to minimize the influence of different genetic population structures. A challenge lies in 


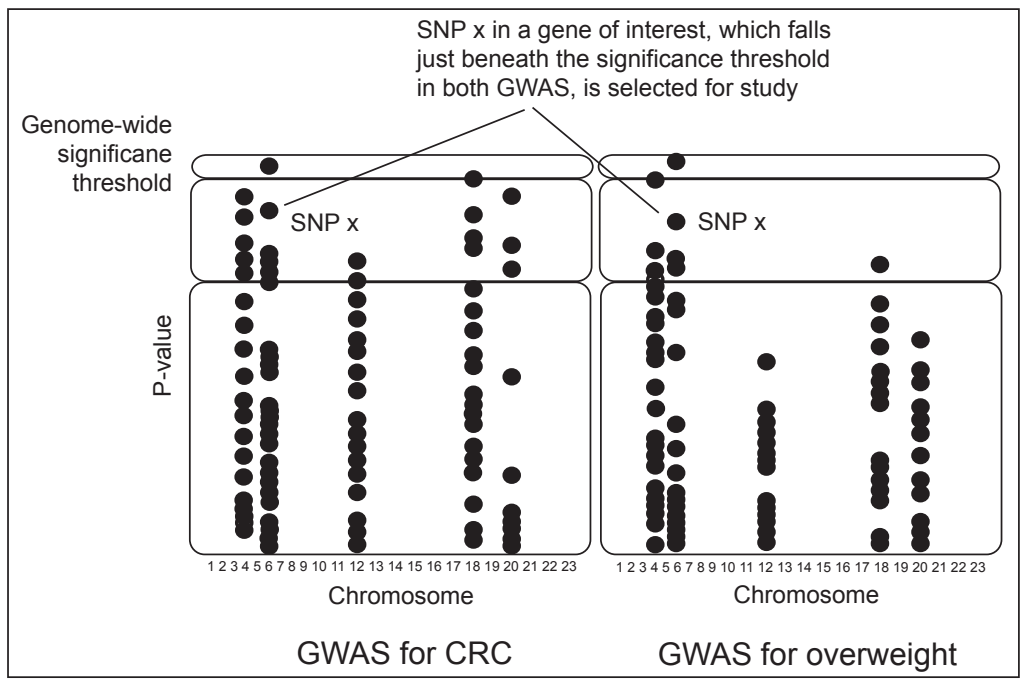

Figure 1. Illustration of a SNP selection strategy based on GWAS for CRC and overweight.

specifying the 'top region' of a GWAS, which could, for example, be operationalized as to comprising those SNPs for which the associated $P$-value falls within the top $1 \%$ of highest $P$-values. With respect to step 4 , recent research by Minelli et al. ${ }^{32}$ has shown that SNPs linked with the phenotype of interest in a meta-analysis or in more than one study were most likely true positives (relative probability: 21.9), followed by SNPs in a functional domain or in a gene previously associated with the phenotype in mouse models (relative probabilities: 9.6 and 9.5, respectively). In this case-control study, replicated GWAS hits for 7 common disease traits were treated as 'cases' representative of true positives and random SNPs were treated as 'controls'. Whether the newly suggested SNP selection strategy is feasible remains to be tested.

\section{A.4 Statistical methods for analyzing higher- order combinations of $\mathrm{G}$ and $\mathrm{E}$}

When analyzing higher-order combinations of multiple genetic variants and dietary or lifestyle factors, one is faced with the curse of dimensionality. This curse entails the occurrence of empty cells or cells with few individ- uals, and a subsequently limited statistical power. However, it is probable that it takes several SNPs in interaction with each other and the environment to produce complex disease, as most single SNPs have been shown to confer only minor risks (1.5 or less). ${ }^{30}$

Parametric approaches, such as logistic regression, appear to be the main statistical analysis method in GxE interaction studies in CRC (Table 1). There are several manners in which higher-order combinations of genetic variants and dietary or lifestyle factors can be modeled using parametric approaches. Firstly, higher-order interactions may be modeled directly, but sample sizes will often be too small to maintain sufficient power. An alternative is to model interactions between haplotypes and dietary or lifestyle factors. In these instances, a choice regarding the inheritance model of the SNP or the reference haplotype is necessary, but this may be difficult. A third possibility, which allows studying the combined effects of loci on different chromosomes, is to model interactions between the cumulative number of genetic risk variants and dietary or lifestyle factors. A logical extension would be to model interactions between the cumulative number 
of genetic risk variants and the cumulative number of dietary and lifestyle risk factors. A specific limitation of cumulative approaches is the potential accumulation of measurement errors, which could result in serious misclassification. It is therefore even more important than usual to ensure the validity of the measurements. With respect to genotyping, this may be done by excluding samples with low call rates as well as SNPs with weak reproducibility in duplicate samples and SNPs not in Hardy Weinberg Equilibrium. ${ }^{33}$

Next to parametric approaches, nonparametric machine learning methods have been gaining interest. These approaches have the advantage of being able to deal with large numbers of variables relative to the number of individuals, while allowing for higher-order interactions. In particular tree-based techniques and multifactor dimensionality reductionbased (MDR) techniques are thought interesting in the context of GxE interaction studies. ${ }^{2}$

With respect to tree-based techniques, random forest (RF) analysis has received most attention. RF analysis builds on Classification and Regression Tree (CART) methodology, as it uses a recursive-partitioning algorithm that, at each node, searches for a value on a particular factor (e.g. a BMI $\left.\geq 25 \mathrm{~kg} / \mathrm{m}^{2}\right)$ that will split individuals into the most homogenous groups possible with respect to disease status. RF analysis involves bagging, which means that trees are built on bootstrap samples from the whole sample. The out-of-bag individuals are used to calculate the classification error of the model, as well as to calculate variable importance measures through permutation testing. The discriminating feature of RF analysis is that the algorithm is only allowed to choose from a random subset of variables at each split. In this way, weakly correlated classification trees are generated, which in aggregate have demonstrated to be a strong classifier. $2,34,35$ However, it must be noted that, while
RF analysis allows for interactions, it does not model interactions per se, and it has been shown weak at identifying interaction models involving variables without marginal effects. 2,36

MDR-based methods continue to be developed. The essence of these methods is that individuals are pooled into high- and low-risk groups, as based on the ratio between cases and controls in each cell in a contingency table. In this way, a new, binary variable is derived, which is evaluated for its ability to classify and predict disease status. ${ }^{37}$ MDR-based methods include a 10 -fold cross-validation procedure that is repeated 10 times in order to find the best model possible, i.e. a robust model that is not merely an artefact of the specific structure of the data from which it is derived. Models are evaluated based on the prediction error and on the cross-validation consistency (CVC). The CVC reflects the number of times the same set of factors was identified across the 10 data subsets (averaged over the 10 cross-validation trials). The CVC theoretically ranges between $1-10$. The best final model is considered to be the model for which the CVC is highest and the prediction error smallest. If these measures point to different models, the most parsimonious model is chosen. The significance of the prediction error and CVC is derived by comparing the estimated prediction error and CVC from the observed data to the distribution of these measures under the null hypothesis of no association, as derived from 1,000 permutations. ${ }^{37-39}$ Advantages of MDR methods include an optimization of power, while allowing for empty cells. In addition, no marginal effects of variables are required. ${ }^{37}$ However, difficult to interpret models may result. ${ }^{35}$

\section{A.5 Summarizing remarks}

For the study of GxE interactions aimed at elucidating pathways linking dietary and life- 
style factors to CRC, myriad designs have been applied. A hypothesis-based approach (using biological knowledge for gene selection) within existing, well-designed epidemiological studies allows for the use of available dietary and lifestyle information without loss of detail. With respect to the SNP selection in hypothesis-based studies, GWAS may turn out useful repositories for pre-selecting SNPs on their likelihood of interacting with the dietary or lifestyle factor of interest. However, such a procedure may only be possible for certain traits, for example overweight or height. Furthermore, the analysis of higher-order combinations of SNPs and dietary or lifestyle factors must be encouraged. 


\section{References}

1. Thomas, D. Methods for investigating geneenvironment interactions in candidate pathway and genome-wide association studies. Annu Rev Public Health 31, 21-36 (2010).

2. Aschard, H. et al. Challenges and opportunities in genome-wide environmental interaction (GWEI) studies. Hum Genet 131, 1591-1613 (2012).

3. Smith, P. G. \& Day, N. E. The design of casecontrol studies: the influence of confounding and interaction effects. Int J Epidemiol 13, 356-365 (1984).

4. KEGG Pathway Maps. At: http://www.genome.jp/kegg/kegg3a.html. Accessed: April 10, 2013.

5. Figueiredo, J. C. et al. Genotype-environment interactions in microsatellite stable/microsatellite instability-low colorectal cancer: results from a genome-wide association study. Cancer Epidemiol Biomarkers Prev 20, 758-766 (2011).

6. Murcray, C. E., Lewinger, J. P. \& Gauderman, W. $\mathrm{J}$. Gene-environment interaction in genome-wide association studies. Am J Epidemiol 169, 219-226 (2009).

7. Siegert, S. et al. Genome-wide investigation of gene-environment interactions in colorectal cancer. Hum Genet 132, 219-231 (2013).

8. Hutter, C. M. et al. Characterization of geneenvironment interactions for colorectal cancer susceptibility loci. Cancer Res 72, 2036-2044 (2012)

9. Kocarnik, J. D. et al. Characterization of 9p24 risk locus and colorectal adenoma and cancer: geneenvironment interaction and meta-analysis. Cancer Epidemiol Biomarkers Prev 19, 3131-3139 (2010).

10. Zhong, R. et al. Genetic variations in the TGF $\beta$ signaling pathway, smoking and risk of colorectal cancer in a Chinese population. Carcinogenesis 34, 936-942 (2013).

11. Liu, L. et al. Interactions between genetic variants in the adiponectin, adiponectin receptor 1 and environmental factors on the risk of colorectal cancer. PLoS One 6, e27301 (2011).

12. Joshi, A. D. et al. Red meat and poultry intake, polymorphisms in the nucleotide excision repair and mismatch repair pathways and colorectal cancer risk. Carcinogenesis 30, 472-479 (2009).

13. Wang, J. et al. Carcinogen metabolism genes, red meat and poultry intake, and colorectal cancer risk. Int J Cancer 130, 1898-1907 (2012).

14. Chen, J. et al. A prospective study of Nacetyltransferase genotype, red meat intake, and risk of colorectal cancer. Cancer Res 58, 3307-3311 (1998).
15. Brevik, A. et al. Polymorphisms in base excision repair genes as colorectal cancer risk factors and modifiers of the effect of diets high in red meat. Cancer Epidemiol Biomarkers Prev 19, 3167-3173 (2010).

16. Fan, C. et al. Case-only study of interactions between metabolic enzymes and smoking in colorectal cancer. BMC Cancer 7, 115 (2007).

17. Hansen, R. D. et al. XPA A23G, XPC Lys939Gln, XPD Lys751Gln and XPD Asp312Asn polymorphisms, interactions with smoking, alcohol and dietary factors, and risk of colorectal cancer. $\mathrm{Mu}$ tat Res 619, 68-80 (2007).

18. Hansen, R. D. et al. A haplotype of polymorphisms in ASE-1, RAI and ERCC1 and the effects of tobacco smoking and alcohol consumption on risk of colorectal cancer: a Danish prospective case-cohort study. BMC Cancer 8, 54 (2008).

19. Matsuo, K. et al. One-carbon metabolism related gene polymorphisms interact with alcohol drinking to influence the risk of colorectal cancer in Japan. Carcinogenesis 26, 2164-2171 (2005).

20. Andersen, V., Egeberg, R., Tjønneland, A. \& Vogel, U. Interaction between interleukin-10 (IL-10) polymorphisms and dietary fibre in relation to risk of colorectal cancer in a Danish case-cohort study. BMC Cancer 12, 183 (2012).

21. Pande, M., Amos, C. I., Eng, C. \& Frazier, M. L. Interactions between cigarette smoking and selected polymorphisms in xenobiotic metabolizing enzymes in risk for colorectal cancer: A case-only analysis. Mol Carcinog 49, 974-980 (2010).

22. Wu, Y. Z. et al. Application of Crossover Analysis-logistic Regression in the Assessment of Geneenvironmental Interactions for Colorectal Cancer. Asian Pac J Cancer Prev 13, 2031-2037 (2012).

23. Tan, X. L. et al. Genetic polymorphisms in TP53, nonsteroidal anti-inflammatory drugs and the risk of colorectal cancer: evidence for geneenvironment interaction? Pharmacogenet. Genomics 17, 639-645 (2007).

24. Khoury-Shakour, S. et al. Recreational physical activity modifies the association between a common GH1 polymorphism and colorectal cancer risk. Cancer Epidemiol Biomarkers Prev 17, 3314-8 (2008).

25. Khoury, M. J. \& Flanders, W. D. Nontraditional epidemiologic approaches in the analysis of geneenvironment interaction: case-control studies with no controls! Am J Epidemiol 144, 207-213 (1996).

26. Tian, C., Gregersen, P. K. \& Seldin, M. F. Accounting for ancestry: population substructure 
and genome-wide association studies. Hum. Mol. Genet. 17, R143-150 (2008).

27. Hsu, L. et al. Powerful cocktail methods for detecting genome-wide gene-environment interaction. Genet Epidemiol 36, 183-194 (2012).

28. Ioannidis, J. P. Why most published research findings are false. PLoS Med 2, e124 (2005).

29. Theodoratou, E. et al. Systematic meta-analyses and field synopsis of genetic association studies in colorectal cancer. J Natl Cancer Inst 104, 14331457 (2012).

30. Hunter, D. J., Altshuler, D. \& Rader, D. J. From Darwin's finches to canaries in the coal mine-mining the genome for new biology. $N$ Engl J Med 358, 2760-2763 (2008).

31. Hunter, D. J. Lessons from genome-wide association studies for epidemiology. Epidemiology 23, 363-367 (2012).

32. Minelli, C. et al. Importance of different types of prior knowledge in selecting genome-wide findings for follow-up. Genet Epidemiol 37, 205-213 (2013).

33. Attia, J. et al. How to use an article about genetic association: B: Are the results of the study valid? JAMA 301, 191-197 (2009).

34. Random forests - classification description. At: http:/ / www.stat.berkeley.edu/ breiman/
RandomForests/cc_home.htm. Accessed: November 1, 2012.

35. Rothman, $\mathrm{N}$ et al. Molecular epidemiology: principles and practices. Geneva, Switzerland: IARC Scientific Publications, No. 163, 2011.

36. McKinney, B. A., Crowe, J. E., Guo, J. \& Tian, D. Capturing the spectrum of interaction effects in genetic association studies by simulated evaporative cooling network analysis. PLoS Genet 5, e1000432 (2009).

37. Ritchie, M. D. et al. Multifactor-dimensionality reduction reveals high-order interactions among estrogen-metabolism genes in sporadic breast cancer. Am J Hum Genet 69, 138-147 (2001).

38. Ritchie, M. D., Hahn, L. W. \& Moore, J. H. Power of multifactor dimensionality reduction for detecting gene-gene interactions in the presence of genotyping error, missing data, phenocopy, and genetic heterogeneity. Genet Epidemiol 24, 150-157 (2003).

39. Hahn, L. W., Ritchie, M. D. \& Moore, J. H. Multifactor dimensionality reduction software for detecting gene-gene and gene-environment interactions. Bioinformatics 19, 376-382 (2003).

40. Yu, W. et al. GWAS Integrator: a bioinformatics tool to explore human genetic associations reported in published genome-wide association studies. Eur J Hum Genet 19, 1095-1099 (2011). 
Supplemental Table 1. GWAS hits for colorectal cancer, as cited in HuGE Navigator ${ }^{40}$

\begin{tabular}{ccc}
\hline Variant & Gene & Region \\
\hline rs6687758 & DUSP10 & $1 \mathrm{q} 41$ \\
rs6691170 & DUSP10 & $1 \mathrm{q} 41$ \\
rs10936599 & MYNN & $3 \mathrm{q} 26.2$ \\
rs1321311 & CDKN1A & $6 \mathrm{p} 21.2$ \\
rs7758229 & SLC22A3 & $6 \mathrm{q} 25.3$ \\
rs16892766 & EIF3H & $8 \mathrm{q} 23.3$ \\
rs10505477 & ORF DQ515897 & $8 \mathrm{q} 24.21$ \\
rs6983267 & Intergenic & $8 \mathrm{q} 24.21$ \\
rs7014346 & POU5FIP1 HsG57825 DQ515897 \\
rs10795668 & Intergenic & $8 \mathrm{q} 24.21$ \\
rs3824999 & POLD3 & $10 \mathrm{p} 14$ \\
rs3802842 & Intergenic & $11 \mathrm{q} 13.4$ \\
rs11169552 & LARP4 DIP2 & $11 \mathrm{q} 23.1$ \\
rs4444235 & BMP4 & $12 \mathrm{q} 13.12$ \\
rs4779584 & Intergenic & $14 \mathrm{q} 22.2$ \\
rs9929218 & CDH1 & $15 \mathrm{q} 13.3$ \\
rs4939827 & SMAD7 & $16 \mathrm{q} 22.1$ \\
rs10411210 & RHPN2 & $18 \mathrm{q} 21.1$ \\
rs961253 & Intergenic & $19 \mathrm{q} 13.11$ \\
rs4925386 & LAMAS & $20 \mathrm{p} 12.3$ \\
rs5934683 & SHROOM2 & $20 \mathrm{q} 13.33$ \\
\hline \hline
\end{tabular}




\section{A brief discussion on energy balance and colorectal cancer}

Since colorectal cancer (CRC) affects millions of people worldwide ${ }^{1}$ and, next to that, obesity prevalences are of growing concern, ${ }^{2}$ a better characterization of indicators of energy balance as risk factors for CRC is important. The aim of this thesis was to study the sexand subsite-specific associations between indicators of energy balance and CRC risk, and to study the role of the insulin-like growth factor pathway and the CpG island methylator phenotype (CIMP) in linking energy balance to CRC development. Additionally, because etiology and prognosis are on the same continuum, and because CIMP and its correlate microsatellite instability (MSI) are thought to hold prognostic value, a prognostic study was conducted, taking into account the overlap between MSI, CIMP, and chromosomal instability (CIN). MSI, CIMP, and CIN are the three main instability types known in CRC today.

\section{8в.1 Main findings}

A qualitative summary of the findings described in chapters 2-6 of this thesis is given in Table 1 for men and in Table 2 for women. Table 1 shows that, although it may have appeared from marginal associations in men that body mass index (BMI), trouser size, and early life energy restriction were differentially associated with cancer risk in colon subsites, combined effects of these factors and genetic variants in genes related to the insulin-like growth factor (IGF) pathway revealed associations with both proximal and distal colon cancer risk. Height was not associated with risk in men, unless investigated in combination with IGF pathway-related polymorphisms. In this analysis, a joint influence was observed on distal colon cancer risk. Marginal associations furthermore existed between physical activity, BMI at age 20 and distal colon cancer risk in men; BMI change was not associated with any CRC endpoint.

In women as compared to men, early life energy restriction appeared to be less important in determining CRC risk, whereas height and physical activity appeared to be more important (Table 2). Height and physical activity were marginally associated with the risk of distal colon cancer and the risk of cancer in all colorectal subsites, respectively. In addition, when physical activity was considered in combination with trouser/skirt size, joint effects were observed on the risk of cancer across subsites. This finding was remarkable since trouser/skirt size was unrelated to CRC risk in women when studied marginally. Joint effects of indicators of energy balance and genetic variants in genes related to the IGF pathway on CRC risk were generally not evident in women.

With respect to molecular endpoints, the observed associations were always in the hypothesized direction. Associations with indicators of energy balance were specific to CRCs characterized by several methylated IGFBP genes, although mostly not specific to tumors characterized by the $\mathrm{CpG}$ island methylator phenotype (CIMP) (Tables 1 and 2). The exception was BMI at age 20, which was found to be a stronger risk factor for CIMP tumors than non-CIMP tumors, as indicated by a statistically significant test for heterogeneity. The latter may have been a chance finding, yet fitted with previous data from the Netherlands Cohort Study (NLCS) regarding early life energy restriction and CIMP tumors (included in Tables 1 and 2 for completeness). It was therefore hypothesized that timing of exposure may influence epigenetic mechanisms, specifically CIMP. 


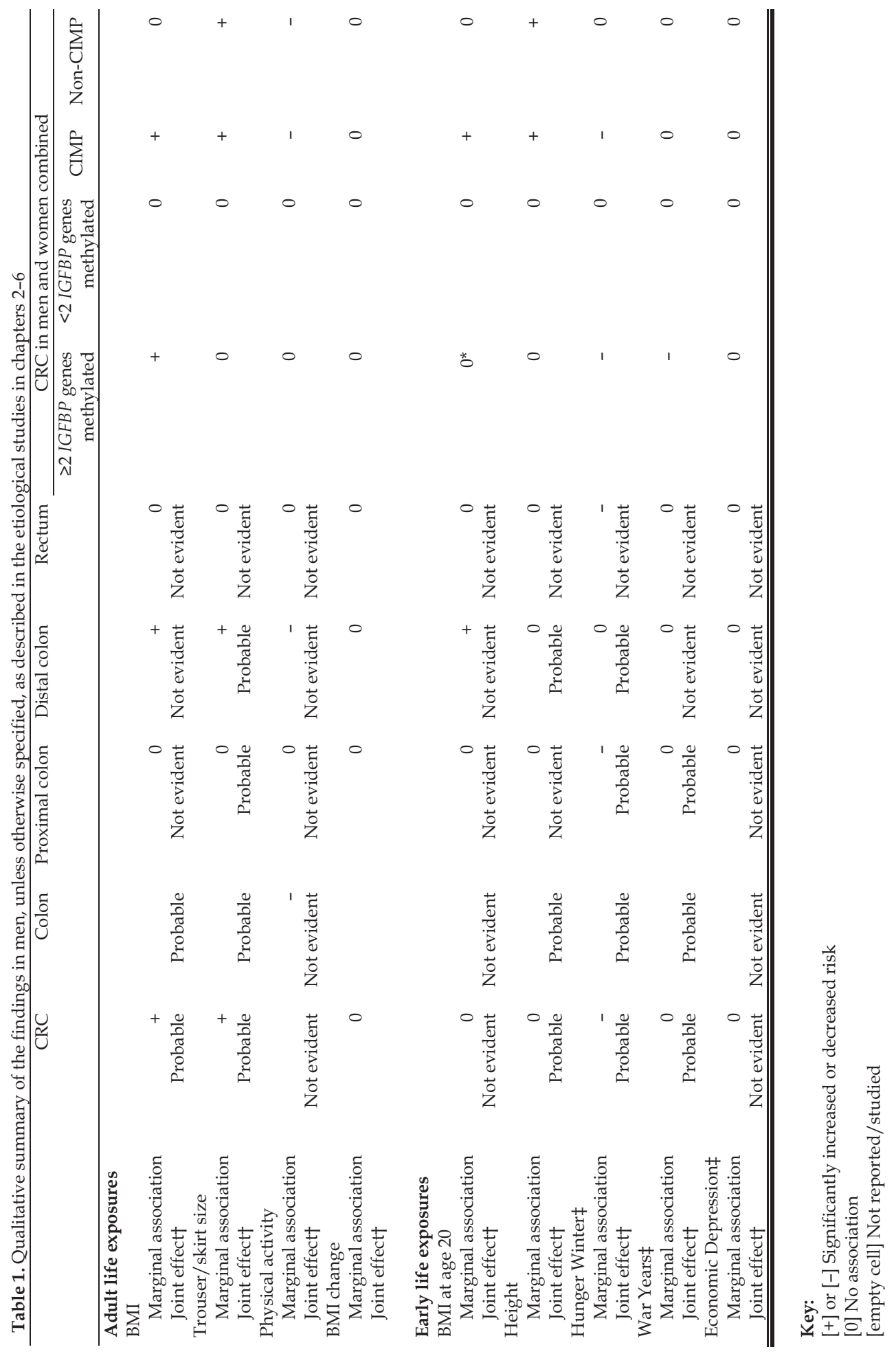

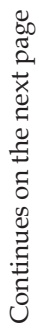




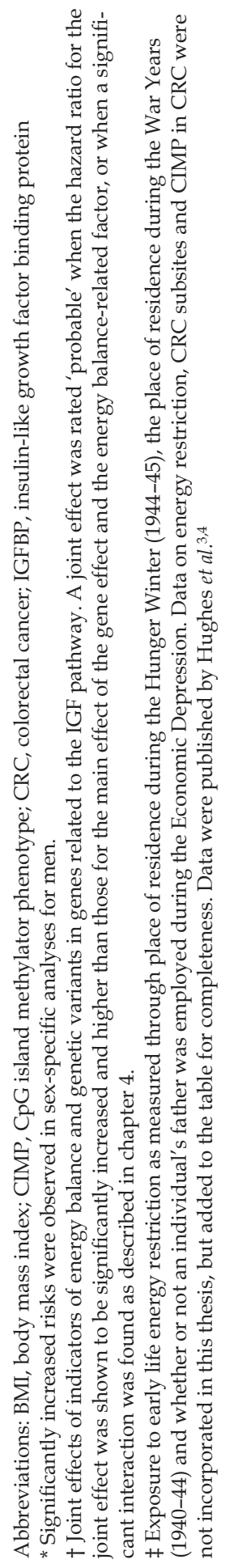




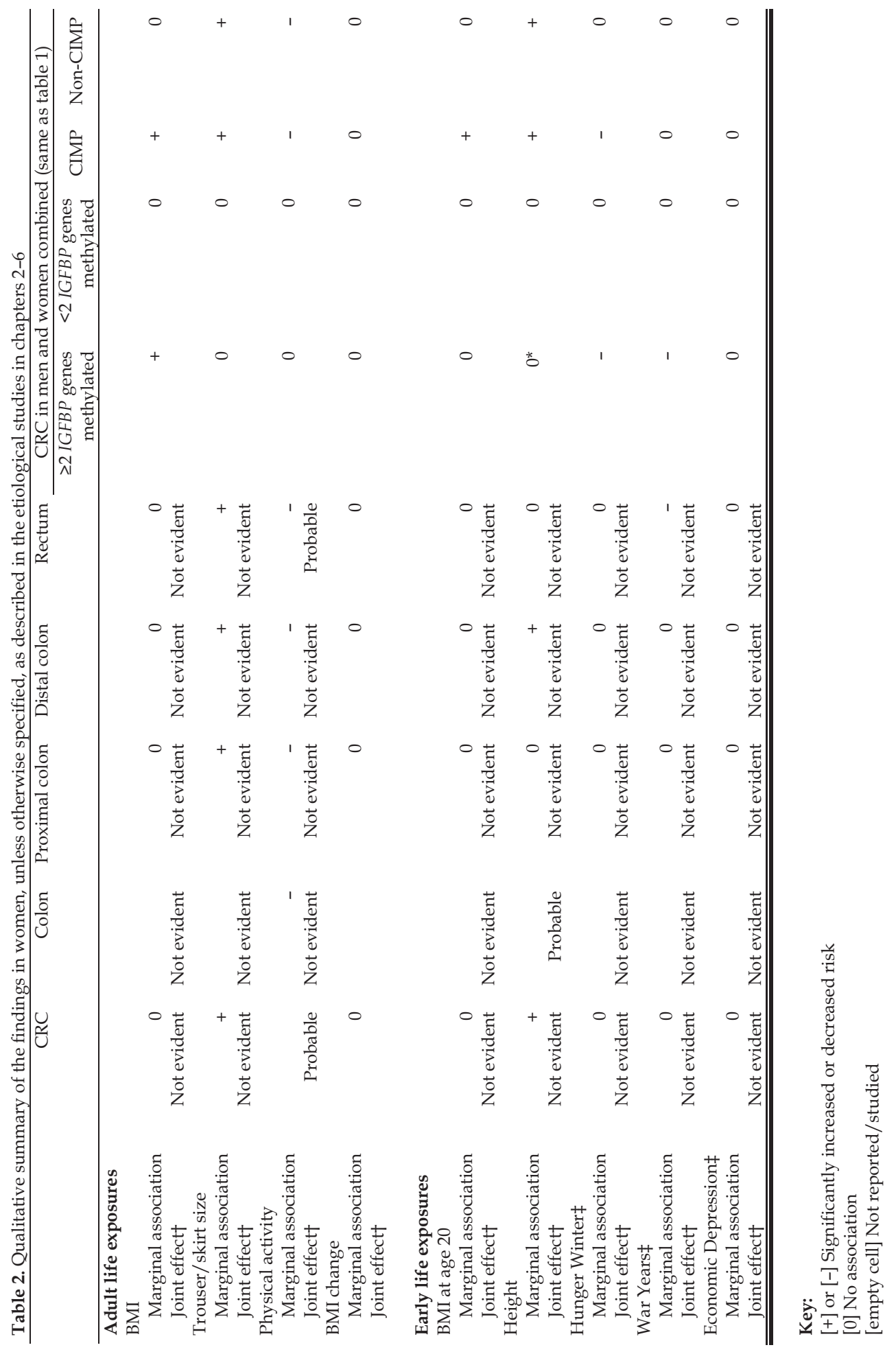

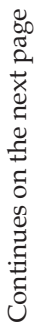




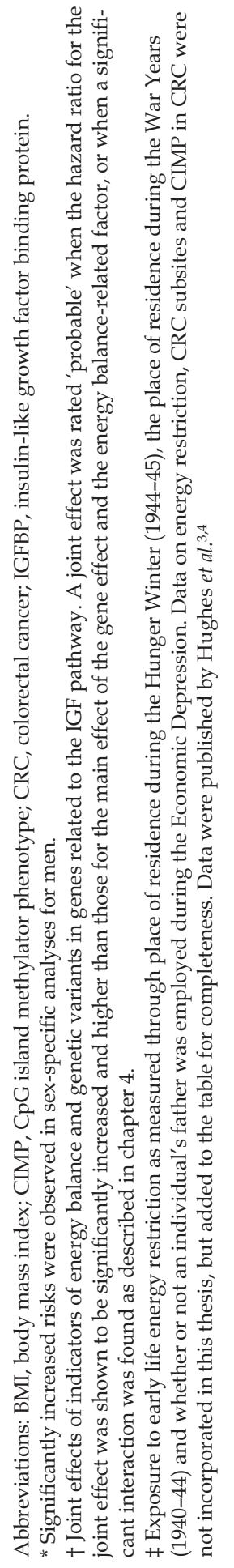


Finally, chapter 7 - of which the results were not included in Tables 1 and 2-showed that specific instability types in CRC, as based on MSI, CIMP, and CIN, hold differential prognostic value that may vary over time. A substantial number of tumors were triple negative for the investigated instability types, and it remains to be seen whether these constitute a truly novel group.

\section{B.2 Interpretation of findings}

With respect to the findings described here, two specific points call for elaboration, relating to the role of the IGF pathway and methylation of specific genes in a pathway versus CIMP. Firstly, the IGF pathway was shown to link indicators of energy balance to proximal and distal colon cancer risk in men as indicated by joint effects of indicators of energy balance and genetic variants in genes related to the IGF pathway. Hazard ratios were between 1.9 and 2.5 for men with a larger trouser size, men who were among the tallest individuals in the NLCS and men without early life energy restriction, if they were in the highest tertile of the sum of unfavorable alleles, indicating moderately increased risks. Combined effects were not evident in women, and it was hypothesized in chapter 4 that this may be because female reproductive hormones counteract insulin resistance. ${ }^{5}$ The role of the IGF pathway in linking energy balance to CRC risk was reiterated by the finding that associations between indicators of energy balance and CRC risk by IGFBP methylation status were confined to CRCs characterized by several methylated IGFBP genes in men and women combined (chapter 5). In this study, hazard ratios were repeatedly in the 1.5-2.0 range, also indicating moderately increased risks.

With respect to IGFBP methylation and CIMP, the question remains whether these are two different phenomena or whether IGFBP methylation is a marker for CIMP. Cross tabulation of tumor instability groups with the extent of IGFBP methylation in chapter 5 showed that $36.8 \%$ of tumors with three methylated IGFBP genes were microsatellite stable non-CIMP tumors. Consequently, IGFBP methylation did not seem to be a marker for CIMP. However, it can never be excluded that associations between indicators of energy balance and specific molecular alterations in CRC are confounded by (epi)genome-wide changes determining CIMP, ${ }^{6}$ unless the cause of these changes is known. A potential cause of methylation is thought to be inflammation, ${ }^{7}$ and obesity has been associated with a chronic state of lowgrade inflammation. ${ }^{8,9}$ It could therefore be that inflammation has a mediating role in the relationship between obesity and CIMP (and ultimately CRC). Since CIMP is thought to be an early event in tumorigenesis, ${ }^{10}$ associations between early life indicators of energy balance and CIMP in CRC may exist in particular. On the other hand, once methylation of certain genes has occurred, this may render individuals especially susceptible to the effects of an unfavorable energy balance, which may be independent of timing of exposure. In this thesis, for example, associations were observed between adult BMI and IGFBP methylation in CRC.

\section{Strengths and limitations}

The strengths and limitations of the conducted studies, in light of which the current findings should be viewed, have been described in detail in chapters 2-7. In brief, the results described in this thesis were unlikely influenced by selection and information bias due to the prospective design of the NLCS, nor does confounding seem to have been an issue due to the availability of extensive information on dietary and lifestyle factors, which enabled the adjustment for various potential confounders. The chance of false inferences 
due to reverse causality was minimized by repeating analyses excluding the first two years of follow-up, and checking that no essential changes in results occurred. A limitation in the NLCS may have been the single baseline exposure measurement; however, the elderly Dutch population in 1986 is likely to have had relatively stable (dietary) habits. ${ }^{11,12}$ Of specific note is that in most studies numerous associations were studied, increasing the potential for false-positive findings, and a criticism may be that no correction was applied for multiple testing. However, biologically plausible hypotheses motivated the studies in this thesis, and, in interpreting results, it was considered important that associations were repeatedly in the hypothesized direction for the different exposure variables investigated. The availability of detailed exposure information in this respect is a specific strength of the NLCS. Finally, a specific limitation with respect to the studies on molecular endpoints may have been the sample size. This limitation is present in any (cohort) study investigating molecular endpoints because any such study will involve multiple exclusions based on the availability of tumor material and valid assay results, ${ }^{6}$ and most cohort studies were not originally designed to study molecular endpoints. As a result, associations between energy balance and molecular endpoints in CRC by subsite have been understudied. Elucidation of these associations would be very valuable as specific molecular alterations tend to correlate with the tumor occurrence site (chapter 7 and e.g. ${ }^{10,13}$ ).

\section{B.3 Further research}

Future molecular epidemiological studies with respect to energy balance and CRC risk may focus on downstream signalling pathways of the IGF pathway, i.e. the PI3K/Akt/mTOR pathway and the MAP/ERK-kinase pathway, ${ }^{14,15}$ and on potential interactions between IGF pathway-related factors and estrogen-related factors in women (chapter 4 of this thesis). Furthermore, evidence is accumulating that a high BMI and a low level of physical activity may unfavorably influence prognostic outcomes in CRC, 16 and that CRC outcomes associated with BMI may depend on molecular tumor characteristics related to energy balance. ${ }^{6}$ The IGF pathway, investigated in this thesis, has been hypothesized to be a putative mechanism linking BMI and physical activity to CRC survival, 16 but the role of the IGF pathway has not yet been investigated. Molecular epidemiological studies investigating this hypothesis will be faced with having to account for changes in body composition after cancer diagnosis ${ }^{17}$ and interactions with cancer therapy (chapter 7).

\section{B.4 Conclusions}

Understanding disease pathways is important as it may improve preventive and therapeutic strategies. This thesis adds to a line of molecular epidemiological studies suggesting that the IGF pathway links indicators of energy balance to CRC risk. In particular combined effects on proximal and distal colon cancer risk were observed of trouser size, height, and early life energy restriction on one hand and IGF pathway-related SNPs on the other hand in men. Notable is that the analysis of combined effects revealed associations with proximal colon cancer risk that were not evident marginally. The role of the IGF pathway was reiterated by that methylation of IGFBP genes was shown to link indicators of energy balance to CRC risk, although it is unclear whether methylation in these specific genes is of importance or whether a general methylator phenotype is underlying associations. It is probable that specific pathways, such as the IGF pathway, link energy balance to CRC risk against a background of general disease pathways such as MSI, CIMP, and CIN. The relative contributions of different pathways may lead to differences in associations be- 
tween indicators of energy balance and CRC risk in men and women, and may cause tumors to occur at specific anatomical sublocalizations. The timing of exposure to a positive or negative energy balance may be important with respect to which pathways become important. 


\section{References}

1. International Agency for Research on Cancer (IARC). GLOBOCAN 2008. At: http://globocan.iarc.fr/factsheet.asp. Accessed: May 10, 2013.

2. WHO | Obesity and overweight. WHO. At: http://www.who.int/mediacentre/factsheets/fs 311/en. Accessed: May 10, 2013.

3. Hughes, L. A. et al. Early life exposure to famine and colorectal cancer risk: a role for epigenetic mechanisms. PLoS One 4, e7951 (2009).

4. Hughes, L. A. et al. Childhood and adolescent energy restriction and subsequent colorectal cancer risk: results from the Netherlands Cohort Study. Int J Epidemiol 39, 1333-1344 (2010).

5. Rondini, E. A., Harvey, A. E., Steibel, J. P., Hursting, S. D. \& Fenton, J. I. Energy balance modulates colon tumor growth: Interactive roles of insulin and estrogen. Mol Carcinog 50, 370-382 (2011).

6. Ogino, S., Chan, A. T., Fuchs, C. S. \& Giovannucci, E. Molecular pathological epidemiology of colorectal neoplasia: an emerging transdisciplinary and interdisciplinary field. Gut 60, 397-411 (2011)

7. Suzuki, H. et al. Roles and causes of abnormal DNA methylation in gastrointestinal cancers. Asian Pac J Cancer Prev 7, 177-185 (2006).

8. Dossus, L. \& Kaaks, R. Nutrition, metabolic factors and cancer risk. Best Pract Res Clin Endocrinol Metab 22, 551-571 (2008).
9. Monteiro, R. \& Azevedo, I. Chronic inflammation in obesity and the metabolic syndrome. Mediators Inflamm 2010, (2010).

10. Issa, J. P. Colon cancer: it's CIN or CIMP. Clin Cancer Res 14, 5939-40 (2008).

11. Van den Brandt, P. A. et al. A large-scale prospective cohort study on diet and cancer in The Netherlands. J Clin Epidemiol 43, 285-295 (1990).

12. Goldbohm, R. A. et al. Reproducibility of a food frequency questionnaire and stability of dietary habits determined from five annually repeated measurements. Eur J Clin Nutr 49, 420-9 (1995).

13. Jass, J. R. Classification of colorectal cancer based on correlation of clinical, morphological and molecular features. Histopathology 50, 113-30 (2007).

14. Arcidiacono, B. et al. Insulin resistance and cancer risk: an overview of the pathogenetic mechanisms. Exp Diabetes Res 2012, 789174 (2012).

15. Weijenberg, M. P. et al. The mTOR Pathway and the role of energy balance throughout life in colorectal cancer etiology and prognosis: unravelling mechanisms through a multidimensional molecular epidemiologic approach. Curr Nutr Rep 2,1926 (2013).

16. Vrieling, A. \& Kampman, E. The role of body mass index, physical activity, and diet in colorectal cancer recurrence and survival: a review of the literature. Am J Clin Nutr 92, 471-490 (2010).

17. Tsai, S. Importance of lean body mass in the oncologic patient. Nutr Clin Pract 27, 593-598 (2012). 


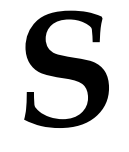

\section{SUMMARY/SAMENVATTING}

Colinda CJM Simons 


\section{SUMMARY}

'No two colorectal cancers are the same.'

The above concept has become clear in recent years and indicates the complexity of colorectal cancer as a disease. Here, the focus is on energy balance-related risk factors for colorectal cancer such as overweight and a low level of physical activity. This thesis shows that energy balance influences colorectal cancer risk via specific disease pathways in men and women, resulting in tumors at specific sublocalizations and with distinct molecular characteristics. Different tumor subtypes were shown to differentially influence prognosis.

An influence of indicators of energy balance, such as overweight and physical activity, on colorectal cancer risk has been shown quite convincingly in the literature. How risk differs between men and women and according to anatomical sublocalization in the colorectum is still relatively unclear, as are the disease pathways through which effects occur. We distinguished between proximal colon, distal colon, and rectal tumors, and set out to clarify associations between indicators of energy balance and the risk of colorectal cancer by anatomical sublocalization in men and women. With respect to disease pathways, we primarily investigated the insulin-like growth factor pathway (IGF) as a pathway through which energy balance potentially influences risk. The IGF pathway is a putative pathway via which energy balance influences colorectal cancer risk, because it regulates growth in the human body, and may also influence malignant growth. Blood levels of factors involved in the IGF axis have been associated with the risk of colorectal cancer and have been shown to be influenced by overweight and physical activity. It has furthermore been reported in literature that diabetics - who have gone through a state of insulin resistance and asso- ciated hyperinsulinaemia - are at an increased risk of colorectal cancer, which is likely explained by insulin stimulating the production of insulin-like growth factor-1.

Method of study.

All studies described in this thesis were conducted within the Netherlands Cohort Study on diet and cancer. The Netherlands Cohort Study is a large, ongoing cohort study which includes 120,852 men and women who were between 55 and 69 years old in 1986. At baseline, in 1986, all participants completed a questionnaire on dietary and other risk factors for cancer. Participants who reported a history of cancer were excluded in order to ensure all were still at risk of developing a primary cancer. The whole cohort is followed up to identify cancer cases, while a subcohort of 5,000 individuals is followed up to estimate the accumulated person-time at risk. In this thesis, 16.3 years of follow-up were used. Personyears at risk are counted until cancer incidence, death or loss-to-follow-up. The design of the Netherlands Cohort Study allows for the estimation of relative risks associated with a certain exposure.

A unique feature of the Netherlands Cohort Study is that participants were in youth during the Hunger Winter, War Years, and Economic Depression. This enabled the investigation of both ends of the energy balance spectrum by studying indicators of a larger body size in adulthood and at age 20 , as well as energy restriction during youth (which may have resulted in temporary weight loss and a temporary stagnation of growth). Information was also available on (non-)occupational physical activity and sports participation. 
In this thesis, a molecular epidemiological approach to studying disease pathways was possible because a large group of participants returned toenail clippings, which were originally meant for determining selenium status, but turned out to be a valuable DNA source for the genotyping of germline genetic variants. Next to toenail DNA, tumor DNA was isolated for the colorectal cancer cases that occurred in the period 1989-1993. In this tumor DNA, genetic and epigenetic types of instability were analyzed. The inclusion of molecular markers measured in tumor material in epidemiological studies is sometimes referred to as molecular pathologic epidemiology.

\section{Marginal associations.}

How the influence of energy balance-related factors on colorectal cancer risk differs in men and women and by anatomical sublocalization was studied in chapters 2 and 3 . In chapter 2, it was shown that body fatness as indicated by a higher adult body mass index (weight in kilograms divided by height in metres squared), a larger trouser size, and a higher body mass index at age 20 were associated with an increased risk of distal colon cancer in men. Body fatness in women did not increase colorectal cancer risk, unless considered simultaneously with physical activity. Tallness, which may be considered a proxy for exposure to growth factors in youth, was associated with an increased risk of distal colon tumors in women but not men. Exposure to energy restriction in youth was investigated outside the chapters of this thesis, but was shown to be associated with a decrease in proximal colon cancer risk in men and rectal cancer risk in men and women (Hughes et al., 2010). In chapter 3 , physical activity was shown protective of cancer in the distal colon in men and across all tumor subsites in women. Interestingly, occupational physical activity predicted risk best in men and non- occupational physical activity predicted risk best in women. It was discussed that this is likely explained by that these particular measures reflected regular, long-term physical activity in men and women best.

\section{Combined effects of genes and environment.}

From the above observations, it appeared that there were differences in risk between men and women and according to anatomical sublocalization of the tumor. In chapter 4, in which combined effects were studied between indicators of energy balance and genetic variants in growth-regulating genes related to the IGF pathway, sex differences persisted. In this chapter, it was shown that a larger trouser size, an increased height, and the absence of energy restriction in youth in combination with carrying more unfavorable alleles was associated with an increased risk of tumors in the proximal colon (not for height) and distal colon in men but not women. The fact that associations were repeatedly in the direction as hypothesized suggested the involvement of the IGF pathway in men. In women, it was hypothesized that female reproductive hormones may counteract insulin resistance, as based on recent literature findings, rendering the IGF pathway less important. With respect to differences in risk according to sublocalization, findings of increased proximal colon cancer risks in men were noteworthy, seeing that marginal associations did not reveal these. The general absence of associations with rectal tumors might be explained by that other pathways are important with respect to developing rectal tumors. Rectal tumors, for example, are more often characterized by chromosomal instability than more proximally located colorectal cancers (see chapter 7). Possibly, this pathway is less influenced by indicators of energy balance.

In the above analyses of combined effects of energy balance and genetic variants on colo- 
rectal cancer risk, the number of unfavorable alleles, as based on literature, with respect to 18 different single nucleotide polymorphisms (SNPs) in 9 genes related to the IGF pathway were summed into a sum score and divided into tertiles. This approach provided optimal power and integrated information across genes in a pathway. The latter is important considering that most single SNPs have been shown to confer only minor risks and because functional compensation between genes exists. Our selection of SNPs was based on a minimum of two different studies in which the SNP was found associated with colorectal cancer risk, indicators of energy balance, or other relevant variables. In this way, it was unlikely that our sum score was based on false-positive results and it became more likely that we selected SNPs interacting with energy balance. It was discussed in chapter 8 - the general discussion of this thesis - that genome-wide association studies (GWAS) studies might turn out interesting repositories for a hypothesis-based selection of SNPs in the future. On the basis of the results in these GWAS, a decision can be made with respect to which is the unfavorable allele. Alternatively, in future studies, machine learning algorithms may be used to analyze higher-order combinations of SNPs and dietary or lifestyle factors. These techniques do not require any hypothesis about which is the unfavorable allele.

\section{Tumor heterogeneity.}

The results in chapter 5 reiterated the role of the IGF pathway. In this chapter, adult body mass index and energy restriction in youth were shown to be associated with the risk of having a colorectal cancer with two or three methylated insulin-like growth factor binding protein genes in men and women combined. Sex-specific analyses revealed similar associations for height in women, which were not evident in analyses for men and women com- bined. No associations were observed with colorectal cancers with none or one methylated insulin-like growth factor binding protein gene(s). Methylation - i.e. the potential silencing-of genes is thought to be unfavorable if it affects insulin-like growth factor binding protein genes because the encoded proteins inhibit growth by binding to insulin-like growth factors and making the complex too large to reach target tissues. Thus, it was hypothesized that methylation of these genes may render individuals particularly susceptible to the influence of energy balance-related colorectal cancer risk factors on colorectal cancer development. Our findings supported this hypothesis.

Promoter CpG island methylation of multiple tumor suppressor- and DNA repair genes as a generic phenotype in colorectal cancer is referred to as the $\mathrm{CpG}$ island methylator phenotype (CIMP). In chapter 6 , it was shown that adult body size, body size at age 20 , and physical activity were associated with both CIMP and non-CIMP tumors in the direction as hypothesized. Only body mass index at age 20 appeared significantly more strongly associated with CIMP tumors than non-CIMP tumors. Outside the chapters of this thesis, energy restriction in youth was investigated in relation to CIMP status in colorectal tumors, and was found to decrease the risk of CIMP tumors but not non-CIMP tumors (Hughes et al., 2009). These findings together led to the hypothesis that timing of exposure to indicators of energy balance may be of importance in relation to CIMP in colorectal tumors.

Findings with respect to CIMP should be viewed in light of that there exist different definitions of CIMP (Hughes et al., 2012, 2013). We used the well-accepted Weisenberger gene panel to define CIMP, but until the causes of CIMP become clear, proper comparison of findings between studies is hampered. Previously, it has been postulated that in- 
flammation may lead to methylation, and because obesity has been associated with a chronic state of low-grade inflammationCIMP could be mediating associations between body size and colorectal cancer risk. This would fit with our findings of early life indicators of energy balance being more strongly associated with CIMP-tumors than non-CIMP tumors. On the other hand, once the mechanism of CIMP has been turned on, it may render individuals susceptible to colorectal cancer risk factors.

Much of the interest in CIMP is due to the fact that tumors solely characterized by CIMP are known to spiral into a trajectory of poor prognostic outcome. Therefore, in chapter 7 of this thesis, CIMP, its correlated phenotype microsatellite instability (MSI), and chromosomal instability (CIN) were studied in relation to colorectal cancer-related deaths. The results in chapter 7 indicated increased risks of colorectal cancer-related deaths to be associated with having a 'CIMP-only', 'CIMP + CIN', and 'triple negative' tumor as compared with having a 'CIN-only' tumor. Thus, the relative contribution of MSI, CIMP, and CIN differentially influenced prognosis. Associations were confined to the first two years of follow-up, suggesting possible interactions between tumor instability types and therapy response. After late follow-up, MSI tumors were borderline significantly associated with a decreased risk of colorectal cancer-related deaths. This was in accordance with the literature, and since most MSI tumors are also characterized by CIMP, it suggests that MSI may override CIMP. With respect to the group of triple negative tumors found in this study, it remains to be seen whether this group includes covert CIMP or CIN tumors (the majority of tumors are chromosomally unstable), or whether it constitutes a truly novel group.

\section{Conclusion.}

To conclude, this thesis shows that a larger body size increases the risk of colorectal cancer, whereas physical activity decreases risk. Associations were evident in relation to proximal and distal colon cancer risk, but less clear in relation to rectal cancer risk. The influence of indicators of energy balance on colorectal cancer risk may run via the IGF pathway, particularly in men. This pathway may be of importance against a background of general disease pathways such as CIMP. CIMP and the associated phenotypes MSI and CIN in turn influence prognosis. Increasing our understanding of the interplay between energy balance, timing of exposure, and disease pathways linking energy balance to colorectal cancer in men and women is important because this may lead to improvements in preventive and therapeutic strategies. 


\section{SAMENVATTING}

'Geen twee colorectaalkankers zijn hetzelfde.'

Dit concept is de laatste jaren duidelijk geworden en geeft aan dat colorectaalkanker een complexe ziekte is. In dit proefschrift gaat de aandacht uit naar risicofactoren voor colorectaalkanker gerelateerd aan energiebalans, zoals overgewicht en weinig beweging. Dit proefschrift laat zien dat energiebalans het risico op colorectaalkanker via specifieke routes beïnvloedt in mannen en vrouwen en dat dit resulteert in tumoren op specifieke plekken in het colorectum met verschillende moleculaire kenmerken. Het tumorsubtype beïnvloedt de prognose.

Dat indicatoren van energiebalans het risico op colorectaalkanker beïnvloeden, komt overtuigend naar voren uit de literatuur. Het is nog onduidelijk of verbanden verschillen tussen mannen en vrouwen en waar in het colorectum tumoren veelal ontstaan. Tevens is onduidelijk langs welke routes energiebalans het risico op colorectaalkanker beïnvloedt. Wij maakten onderscheid tussen het proximale deel van de dikke darm, het distale deel van de dikke darm en de endeldarm (samen colorectaalkanker) en hebben getracht verbanden tussen indicatoren van energiebalans en het risico op colorectaalkanker bij manen en vrouwen en naar anatomische sublocalisatie te verduidelijken. We onderzochten het insulinegelijkende groeifactorsysteem (IGFsysteem) als mogelijke route waarlangs energiebalans het risico op colorectaalkanker beinvloedt. Het IGF-systeem is een aannemelijke route omdat dit systeem groei reguleert en mogelijk ook kwaadaardige groei beïnvloedt. Aangetoond is dat hoeveelheden van factoren betrokken bij het IGF-systeem, wanneer gemeten in bloed, verband houden met het risico op colorectaalkanker en beïnvloedt worden door de mate van overgewicht en beweging.
Verder is voor diabeten - welke in een staat van insulineresistentie en hyperinsulinemie hebben verkeerd - een verhoogd risico op colorectaalkanker gevonden, wat mogelijk verklaard wordt doordat insuline de productie van insulinegelijkende groeifactor 1 stimuleert.

\section{Studiemethode.}

De studies beschreven in dit proefschrift zijn uitgevoerd binnen de Nederlandse Cohort Studie naar voeding en kanker. De Nederlandse Cohort Studie is een grote cohortstudie waarin 120,852 mannen en vrouwen deelnemen die tussen de 55 en 69 jaar oud waren in 1986. Aan het begin van de studie, in 1986, hebben alle deelnemers een vragenlijst ingevuld over voeding en andere risicofactoren voor kanker. Deelnemers die rapporteerden kanker te hebben (gehad) werden geëxcludeerd, zodat alle deelnemers die in de loop der tijd kanker kregen een primaire (eerste) tumor hadden. Het hele cohort werd opgevolgd om nieuwe kankerpatiënten te identificeren. Tegelijkertijd werd een subcohort van 5,000 deelnemers gevolgd voor een schatting van de opgebouwde persoonstijd. In dit proefschrift is gebruik gemaakt van 16,3 jaar 'follow-up'. Persoonsjaren werden geteld tot het optreden van kanker, overlijden of 'lossto-follow-up'. De studieopzet maakt het mogelijk relatieve risico's voor colorectaalkanker te berekenen voor het al dan niet hebben van een bepaalde blootstelling.

Een uniek kenmerk van de Nederlandse Cohort Studie is dat deelnemers in hun jeugd waren ten tijde van de Honger Winter, Oorlogsjaren en Economische Depressie. Dit stelde ons ertoe in staat beide kanten van het spectrum van energiebalans te onderzoeken door te kijken naar overgewicht op volwassen 
en op 20-jarige leeftijd en door te kijken naar blootstelling aan energierestrictie tijdens de jeugd (wat mogelijk tijdelijk gewichtsverlies en een tijdelijke stagnatie van groei tot gevolg had). Daarnaast was informatie beschikbaar over beweging in en buiten het beroep en over sportdeelname van deelnemers.

In dit proefschrift onderzochten we mogelijke routes waarlangs energiebalans colorectaalkanker beïnvloedt door middel van een moleculair epidemiologische aanpak. We maakten gebruik van ingestuurd teennagelmateriaal dat oorspronkelijk bedoeld was voor het bepalen van seleniuminname, maar ook een goede bron van DNA bleek te zijn voor het bepalen van erfelijke genetische varianten. Naast dit type DNA, is DNA geïsoleerd uit de tumor van colorectaalkankerpatiënten die tussen 1989 en 1993 werden gediagnosticeerd. In dit tumor DNA, zijn genetische en epigenetische vormen van instabiliteit bepaald. Het meenemen van moleculaire markers gemeten in de tumor in epidemiologisch onderzoek wordt ook wel moleculair pathologisch epidemiologisch onderzoek genoemd.

\section{Marginale verbanden.}

Of de invloed van factoren gerelateerd aan energiebalans op de kans op het krijgen van colorectaalkanker verschilt tussen mannen en vrouwen en tussen anatomische sublocalisaties, werd onderzocht in hoofdstuk 2 en 3 . In hoofdstuk 2 kwam naar voren dat meer lichaamsvet-zoals aangegeven door een hogere body mass index op volwassen leeftijd (gewicht in kilo's gedeeld door lichaamslengte in meters in het kwadraat), een grotere broekmaat en een hogere body mass index op 20-jarige leeftijd - verband hield met een verhoogd risico op kanker in het distale deel van de dikke darm bij mannen. Lichaamsvet hield geen verband met het risico op colorectaalkanker bij vrouwen, tenzij bekeken in combinatie met de mate van beweging. Lichaams- lengte was gerelateerd aan een verhoogd risico op kanker in het distale deel van de dikke darm bij vrouwen en mag gezien worden als een proxymaat voor blootstelling aan groeifactoren in de jeugd. Blootstelling aan energierestrictie tijdens de jeugd werd buiten dit proefschrift om onderzocht en hield verband met een verlaagd risico op kanker in het proximale deel van de dikke darm bij mannen en met een verlaagd risico op endeldarmkanker bij mannen en vrouwen (Hughes et al., 2010). Hoofdstuk 3 toonde verder dat beweging een beschermende factor is voor kanker in het distale deel van de dikke darm bij mannen en voor kanker in alle sublocalisaties in het colorectum bij vrouwen. Beweging in het beroep vormde de beste voorspeller van een verlaagd risico bij mannen, terwijl beweging buiten het beroep de beste voorspeller was van een verlaagd risico bij vrouwen. Mogelijk weerspiegelden deze vormen van beweging bij mannen en vrouwen regelmatige beweging gedurende langere tijd het beste.

Gen-omgevings effecten.

Bovenstaande observaties duidden op risicoverschillen tussen mannen en vrouwen en tussen anatomische sublocalisaties. In hoofdstuk 4, waarin gecombineerde effecten van indicatoren van energiebalans en genetische varianten in genen gerelateerd aan het IGFsysteem werden onderzocht, hielden de geslachtsverschillen aan. Het hebben van een grotere broekmaat, een grotere lichaamslengte en niet blootgesteld zijn aan energierestrictie tijdens de jeugd in combinatie met het dragen van meer ongunstige allelen verhoogde het risico op tumoren in het proximale deel van de dikke darm (niet met betrekking tot lengte) en in het distale deel van de dikke darm bij mannen, maar niet bij vrouwen. De richting van de verbanden was zoals verwacht en wees op de betrokkenheid van het IGFsysteem als route waarlangs energiebalans colorectaalkanker beïnvloedt bij mannen. 
Deze route is bij vrouwen mogelijk minder belangrijk, omdat vrouwelijke hormonen insulineresistentie kunnen tegengaan. Wat betreft risicoverschillen tussen sublocalisaties was de bevinding van een verhoogd risico op kanker in het proximale deel van de dikke darm opmerkelijk, aangezien er geen marginale verbanden met kanker in deze sublocalisatie te zien waren. Het niet vinden van verbanden met endeldarmkanker kan erop duiden dat deze tumoren zich langs andere routes ontwikkelen. Tumoren in de endeldarm worden bijvoorbeeld vaker gekenmerkt door chromosomale instabiliteit dan meer proximaal gesitueerde tumoren (zie hoofdstuk 7) en mogelijk wordt deze route minder sterk beïnvloed door indicatoren van energiebalans.

In bovenstaande analyses naar gecombineerde effecten van energiebalans en genetische varianten hebben we het aantal (volgens de literatuur) ongunstige allelen met betrekking tot 18 'single nucleotide polymophisms' (SNPs) in 9 genen gerelateerd aan het IGF-systeem opgeteld. De resulterende somscore werd opgedeeld in tertielen. Deze aanpak gaf een optimale power en stond toe informatie uit diverse genen op dezelfde route te integreren. Integratie van informatie is belangrijk, omdat SNPs op zichzelf slechts kleine veranderingen in risico's met zich meebrengen en er binnen een systeem ook compensatie in functie tussen genen kan plaatsvinden. Onze SNP-selectie was gebaseerd op minstens twee verschillende studies waarin de SNP verband hield met het risico op colorectaalkanker, indicatoren van energiebalans of andere relevante variabelen. Als zodanig was het minder waarschijnlijk dat onze somscore was gebaseerd op fout-positieve bevindingen en werd het waarschijnlijker dat de geselecteerde SNPs ook daadwerkelijk in samenwerking met factoren gerelateerd aan energiebalans een invloed uitoefenden. In de toekomst - zoals beschreven in hoofdstuk 8 -zijn de resultaten van genoomwijde associatiestudies (GWAS) mo- gelijk bruikbaar voor de selectie van SNPs in studies met een duidelijke hypothese over betrokken routes. Op basis van de resultaten in deze GWAS kan worden besloten welk allel ongunstig is. Als alternatief kan gebruik gemaakt worden van 'machine learning' algoritmes om hogere-orde combinaties van SNPs en voedings- en leefstijlfactoren te analyseren. Deze technieken vereisen geen aanname over welk allel het ongunstige allel is.

\section{Tumor heterogeniteit.}

De resultaten in hoofdstuk 5 benadrukten de rol van het IGF-systeem. Body mass index op volwassen leeftijd en energierestrictie tijdens de jeugd hielden in dit hoofdstuk (waarin mannen en vrouwen samen werden onderzocht) verband met het risico op colorectaalkankers gekenmerkt door twee of drie gemethyleerde genen die coderen voor eiwitten die binden aan insulinegelijkende groeifactoren. Geslachtsspecifieke analyses lieten eenzelfde soort verbanden zien voor lengte bij vrouwen; deze verbanden waren verkapt in gecombineerde analyses. Er waren geen verbanden in relatie tot colorectaalkankers met geen of één gemethyleerd(e) gen(en) coderend voor eiwitten die binden aan insulinegelijkende groeifactoren. Methylering - dat wil zeggen mogelijke stillegging - van genen die coderen voor eiwitten die binden aan insulinegelijkende groeifactoren is ongunstig, omdat deze eiwitten groei remmen, doordat ze samen met insulinegelijkende groeifactoren een te groot geheel vormen om weefsels in organen te bereiken. Onze hypothese was daarom dat methylering van deze genen individuen extra vatbaar maakt voor de invloed van indicatoren van energiebalans op het krijgen van colorectaalkanker. Onze bevindingen ondersteunen deze hypothese.

Promotor CpG eiland methylering van meerdere tumorsuppressor- en DNA-herstelgenen komt voor in een subgroep van colorectaal- 
tumoren, welke bekend staan als CIMPtumoren (tumoren met het "CpG island methylator phenotype"). In hoofdstuk 6 vonden we dat volwassen body mass index, body mass index op 20-jarige leeftijd en beweging verband hielden met zowel CIMP-als nietCIMP-tumoren, waarbij de richting van de verbanden was zoals verwacht. Enkel body mass index op 20-jarige leeftijd hield significant sterker verband met CIMP-tumoren dan met niet-CIMP-tumoren. Buiten dit proefschrift om werd energierestrictie tijdens de jeugd in verband gebracht met een verlaagd risico op CIMP-tumoren, maar niet met nietCIMP-tumoren (Hughes et al., 2009). Samen leidden deze resultaten tot de hypothese dat de levensperiode waarin blootstelling aan indicatoren van energiebalans plaatsvindt, mogelijk van belang is in relatie tot CIMP in colorectaaltumoren.

Bevindingen in relatie tot CIMP moeten gezien worden in het licht van dat er verschillende definities van CIMP bestaan (Hughes et al., 2012, 2013). Wij gebruikten het alom gebruikte Weisenberger genpanel om CIMP te bepalen, echter totdat de oorzaken van CIMP duidelijk worden, zal vergelijking van resultaten tussen studies lastig blijven (Hughes et al., 2012, 2013). Het is gesuggereerd dat ontstekingsreacties leiden tot methylering en omdat obesitas verband houdt met een milde, maar chronische vorm van ontsteking - zou CIMP verbanden tussen lichaamsomvang en het risico op colorectaalkanker kunnen mediëren. Dit past bij onze bevindingen dat vroege indicatoren van energiebalans sterker geassocieerd waren met CIMP-tumoren dan met niet-CIMP-tumoren. Aan de andere kant, zodra CIMP als mechanisme is aangezet, kan het individuen vatbaar maken voor risicofactoren voor colorectaalkanker.

Veel van de aandacht voor CIMP als fenotype is te wijten aan het feit dat CIMP-tumoren een slechte prognose kennen. Met dit in gedachte onderzochten we in hoofdstuk 7 CIMP, het eraan gecorreleerde fenotype microsatelliet instabiliteit (MSI) en chromosomale instabiliteit (CIN) in relatie tot aan colorectaalkankergerelateerde sterfte. De resultaten lieten een verhoogd risico op sterfte zien na diagnose met een 'CIMP-only', 'CIMP + CIN' of 'triple negatieve' tumor in vergelijking met een 'CIN-only'-tumor. Specifieke combinaties van MSI, CIMP en CIN leidden dus tot een verschillende prognose. Verbanden bestonden alleen na de eerste twee jaar van follow-up. Vermoedelijk speelden interacties tussen tumorsubtypes en therapierespons een rol. $\mathrm{Na}$ lange follow-up hield het hebben van een MSI-tumor verband met een vrijwel significant verlaagd risico op aan colorectaalkankergerelateerde sterfte. Deze bevinding is in overeenstemming met de literatuur en doet vermoeden dat MSI CIMP overstemt, omdat de meeste MSI-tumoren ook gekenmerkt worden door CIMP. Wat betreft de 'triple negatieve' groep moet verder onderzoek uitwijzen of dit een nieuwe tumorgroep betreft of dat het verkapte CIMP- of CIN-tumoren zijn (CIN-tumoren vormen de grootste groep).

\section{Conclusie.}

Dit proefschrift laat zien dat een grotere lichaamsomvang het risico op colorectaalkanker verhoogt, terwijl beweging dit risico verlaagt. Dit gold in relatie tot kankers in het proximale en distale deel van de dikke darm, maar minder in relatie tot endeldarmkanker. De invloed van factoren gerelateerd aan energiebalans op colorectaalkanker loopt waarschijnlijk via het IGF-systeem, vooral bij mannen. Deze route speelt mogelijk een rol tegen een achtergrond van algemenere ziektemechanismen zoals CIMP. CIMP en de fenotypes MSI en CIN beïnvloeden op hun beurt de prognose. Een beter begrip van het samenspel tussen energiebalans, het tijdstip van blootstelling en de routes waarlangs energiebalans het risico op colorectaalkanker bij mannen en 
vrouwen beïnvloedt, is van belang voor verbeteringen in preventieve en therapeutische strategieën. 
E

Etcetera 
Verschillende personen waren betrokken bij het schrijven van dit proefschrift. Graag wil ik jullie bedanken voor jullie bijdrage op wetenschappelijk vlak, op persoonlijk vlak of allebei. Mocht ik je naam hier vergeten, dan is dit bedankje zeker niet minder welgemeend.

Als eerste Matty. Met jou als promotor en dagelijks begeleider ben ik toch echt wel met mijn neus in de boter gevallen! Wie had dat kunnen denken toen ik me zoveel jaar geleden bij jou aanmeldde voor een stage. Er zijn maar weinig mensen die wetenschappelijk zo sterk zijn, persoonlijk betrokken zijn, tijd weten te maken als er eigenlijk geen tijd is en tot op het laatst kritisch blijven kijken, waardoor het werk toch nog net ietsje beter wordt. Bedankt voor de uitdaging die je me wist te geven de afgelopen jaren en het leuke overleg iedere week. Ik zie uit naar onze verdere samenwerking!

Manon en Frederik-Jan. Jullie hebben je eigen stijl en daarom was het te gek om ook met jullie te mogen samenwerken en te leren. Manon, zoals velen voor mij hebben gezegd, jouw enthousiasme is aanstekelijk. Jij dwong mij ook in twee zinnen te zeggen wat de belangrijkste boodschap was en vandaaruit een artikel te schrijven. Deze 'directheid' heb ik van je afgekeken en probeer ik vaker toe te passen, want het werkt. Frederik-Jan, ook jij weet te enthousiasmeren. Door jou werd ik me ervan bewust dat niet iedereen 'mijn taal' spreekt. Mensen met een andere achtergrond kunnen heel anders naar je werk kijken dan je zelf denkt, maar dat geeft juist nieuwe inzichten.

Piet. Jij was heel nauw betrokken bij dit project. Bedankt voor je aanwezigheid bij overleggen en alle tijd en moeite die jij in mijn begeleiding hebt gestoken. Je weet altijd op de goede momenten sturing te geven en je commentaar op artikelen is altijd 'spot-on'. Ik ken bijna niemand met zo'n heldere en scherpe visie zoals jij.

Leo. Het is bewonderenswaardig hoe jij projecten tot in detail weet uit te denken. Jouw coördinerend vermogen bij de genotypering in dit project was absoluut onmisbaar. Je wetenschappelijke bijdrage aan artikelen en het sparren over hoe om te gaan met al die data zijn zeer gewaardeerd. Bovendien was het samen met Matty heel gezellig in Miami!

Roger. Ook jij was onmisbaar voor de genotypering in dit project. Hoe klein de vraag ook, ik kon altijd even bellen om iets 'moleculairs' te vragen.

Kim E Kim, Leonie en Joy. Achter al die data in de hoofdstukken van dit proefschrift zit een bulk werk in het laboratorium. Deze bulk hebben jullie voor je rekening genomen. Het mag best worden gezegd dat projecten zoals de deze sterk afhankelijk zijn van jullie inzet! Daarbij heeft Leonie (samen met Ivette en Janneke) ook nog eens goed mijn rijkunsten doorstaan tijdens ons tripje naar Hamburg voor een bezoek aan Sequenom $(;)$.

Epidemiologiecollega's. Bedankt voor jullie collegialiteit! In het bijzonder degenen die samen met Matty en mij meededen aan de Ride for the Roses in 2010. Heel sportief! In het bijzonder ook Harry, Jos, Sacha, Jolanda en Yvonne. Jullie ondersteuning is geweldig!

Pathologie- en toxicologiecollega's. Bedankt dat jullie mij wegwijs maakten op jullie afdelingen. Ik wil vooral ook Audrey en Marie-Claire bedanken voor alle hulp bij het plannen van afspraken. Zonder jullie hulp en die van Jolanda en Yvonne was dat niet gelukt. 
(Oud-)aio's en 'jong-epid'. Anne, Audrey, Brenda, Carin, Carolina, Catherine, Denise, Dianne, Dorthe, Eline, Emmylou, Esther, Fleur, Huub, Ivette, Janneke, Jessie, José, Judith, Karolina, Milan, Mirjam, Nadine, Petra, Rachel, Sarah, Sander van Kuijk, Sander de Kort, Stefan, Stefanie en Vivian wil ik bedanken voor alle gezellige gesprekken op het werk, congressen en cursussen. Voor velen in dit lijstje geldt: lunchen, koffiedrinken, uit eten, koken bij iemand thuis, een $80-90$ s party, jullie draaien je hand er niet voor om $;$. Diegenen onder jullie die nog moeten promoveren, wens ik veel succes bij de afronding van hun boekje.

Anke en Nadine, mijn paranimfen. Hoeveel wijntjes hebben wij niet al gedronken? Ik hoop dat er nog meer zullen volgen, niet per se vanwege de wijn, maar meer vanwege de gezelligheid. Op vriendschap!

Laura $\mathcal{E}$ Richard. Laura, you started off as my roommate and coauthor in DEB1 and ended up being one of my best friends! You have a unique perspective on life and your Canadian view on distance made me realize distance only exists in our minds. I also love your abbreviations: TTYS, I won't go MIA anymore! To Richard: you didn't think I would do it, did you? I bet you this time 2012 wasn't the last time I set foot in Toronto!

Ruth $\mathcal{E}$ Paul. Thanks for the lovely vacation in Cornwall/Devon! I visit far too little... mèr d'r is eigelijk niks leuker dan midden in Londen biej te praote in ut plat!

Andere vrienden en familieleden waarvan vele aan de 'Siem-kant': bedankt! Ik noem geen namen, omdat ik bang ben mensen te vergeten, maar het is altijd fijn als mensen interesse tonen in wat je doet, al blijft het moeilijk het 'epi-de-mio-loog-zijn' uit te leggen.

Pap $\mathcal{E}$ mam. Soms moet je niet veel hoeven zeggen... bedankt.

\section{Colinda}




\section{-ABOUT THE AUTHOR}

Colinda Simons was born on December $14^{\text {th }}, 1983$ in Roermond, the Netherlands. After completing secondary school (Gymnasium, English language trajectory) at the Bisschoppelijk College Broekhin in Roermond in 2002, she studied Health Sciences at Maastricht University in Maastricht, the Netherlands. Colinda obtained her BSc. degree in Health Sciences (cum laude) three years later. In 2007, Colinda enrolled in the Epidemiology master's program at Maastricht University. She completed an internship in the department of Epidemiology at Maastricht University, and obtained her MSc. degree (cum laude) in 2008. For her thesis, she authored an article on dietary flavonoid intake and colorectal cancer risk in the Netherlands Cohort Study, which was published in the International Journal of Cancer. After graduating, Colinda was employed as a junior researcher on the KIWA Water and Health project at Maastricht University for five months. During this time, Colinda wrote a research report and article on fluid intake and colorectal cancer risk in the Netherlands Cohort Study. Then, from 2009 to 2013, Colinda worked on the studies described in this thesis. Colinda will continue to work on topics related to energy balance and colorectal cancer at the department of Epidemiology, and intends to gain experience in machine learning techniques by visiting experts abroad. 


\section{—LIST OF PUBLICATIONS}

Ready for submission:

1. Simons, CCJM, Schouten, LJ, Godschalk, RWL, van Engeland, M, van den Brandt, PA, van Schooten, FJ, Weijenberg, MP. Energy balance and unfavorable alleles in genes related to the IGF pathway jointly influence colon cancer risk in men.

2. De Kort, S, Simons, CCJM, van den Brandt, PA, Goldbohm, RA, Arts, IAW, de Bruïne, AP, Janssen-Heijnen, ML, Sanduleanu, S, Masclee, AAM, Weijenberg, MP. Diabetes mellitus type 2 and subsite-specific colorectal cancer risk: results from the Netherlands Cohort Study.

Submitted:

3. Simons, CCJM, van den Brandt, PA, Stehouwer, CDA, van Engeland, M, Weijenberg, MP. Energy balance influences the risk of having a colorectal tumor with methylated insulin-like growth factor binding protein genes.

Appeared:

4. Simons CCJM, Hughes LAE, Smits KM, Khalidde Bakker CA, de Bruïne AP, Carvalho B, Meijer, GA, Schouten, LJ, van den Brandt, PA, Weijenberg, MP, van Engeland, M. A novel classification of colorectal tumors based on microsatellite instability, the CpG island methylator phenotype and chromosomal instability: implications for prognosis. Ann Oncol 2013;24(8):2048-56.

5. Simons CCJM, Hughes LAE, van Engeland M, Goldbohm RA, van den Brandt PA, Weijenberg MP. Physical activity, occupational sitting time, and colorectal cancer risk in the Netherlands cohort study. Am J Epidemiol 2013;177(6):514-30.

6. Weijenberg MP, Hughes LAE, Bours MJL, Simons CCJM, van Engeland M, van den Brandt PA. The mTOR pathway and the role of energy balance throughout life in colorectal cancer etiology and prognosis: unravelling mechanisms through a multidimensional molecular epidemiologic approach. Curr Nutr Rep 2013;2(1):19-26.
7. Hughes LAE, Simons CCJM, van den Brandt PA, Goldbohm RA, van Engeland M, Weijenberg MP. Body size and colorectal cancer risk after 16.3 years of follow-up: an analysis from the Netherlands Cohort Study. Am J Epidemiol 2011;174(10):1127-39.

8. Hughes LAE, Simons CCJM, van den Brandt PA, Goldbohm RA, de Goeij AF, de Bruïne AP, van Engeland, M, Weijenberg, MP. Body size, physical activity and risk of colorectal cancer with or without the $\mathrm{CpG}$ island methylator phenotype (CIMP). PLoS One 2011;6(4):e18571.

9. Simons CCJM, Schouten LJ, Weijenberg MP, Goldbohm RA, van den Brandt PA. Bowel movement and constipation frequencies and the risk of colorectal cancer among men in the Netherlands Cohort Study on diet and cancer. Am J Epidemiol 2010;172(12):1404-14.

10. Simons CCJM, Leurs LJ, Weijenberg MP, Schouten LJ, Goldbohm RA, van den Brandt PA. Fluid intake and colorectal cancer risk in the Netherlands Cohort Study. Nutr Cancer 2010;62(3):30721.

11. Simons CCJM, Hughes LAE, Arts ICW, Goldbohm RA, van den Brandt PA, Weijenberg MP. Dietary flavonol, flavone, and catechin intake and risk of colorectal cancer in the Netherlands Cohort Study. Int J Cancer 2009;125(12):2945-52.

Other:

12. De Jongh C, Leurs L, Simons CCJM, van den Brandt PA. Epidemiologisch onderzoek naar inname van kraanwater en andere vloeistoffen in relatie tot hart- en vaatziekten en dikke darmkanker. H2O 2010.

13. Simons CCJM, Leurs LJ, Weijenberg MP, Schouten LJ, Goldbohm RA, van den Brandt PA. Fluid intake and colorectal cancer risk in the Netherlands Cohort Study. KWR Watercycle Research Institute. BTO2009.025. 

